\title{
Function of MCPH1 in Neurogenesis
}

\author{
Dissertation
}

zur Erlangung des mathematisch-naturwissenschaftlichen Doktorgrades

"Doctor rerum naturalium"

der Georg-August-Universität Göttingen

vorgelegt von

Ralph Gruber

aus Illertissen

Göttingen 2011 
Mitglieder des Betreuungsausschusses:

Referent: PD Dr. Wilfried Kramer

Koreferent: Prof. Dr. Zhao-Qi Wang

Tag der mündlichen Prüfung: 11. April 2011 


\section{Table of Contents}

Table of Contents

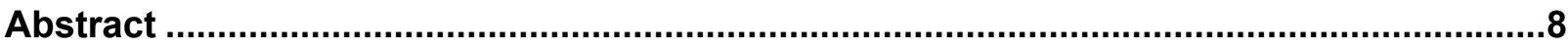

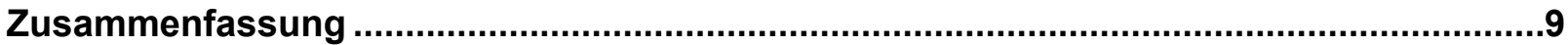

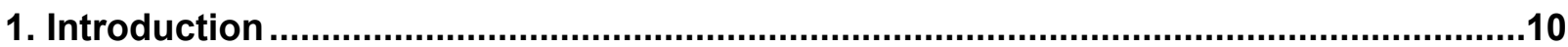

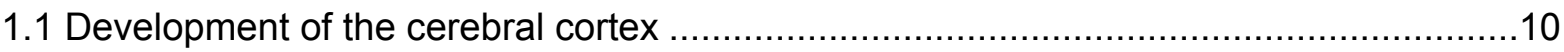

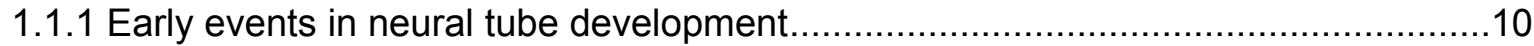

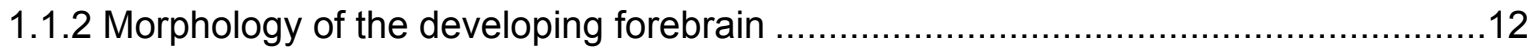

1.1.3 Neuronal stem and progenitor cells of the dorsal telencephalon............................14

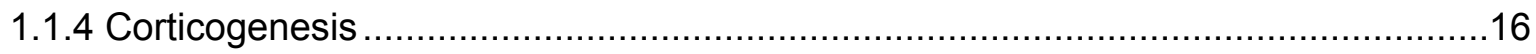

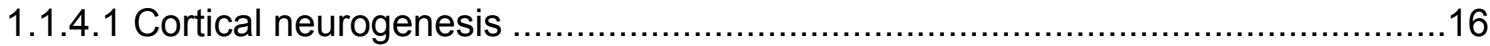

1.1.4.2 Gliogenic phase of cortical development ………...................................17

1.1.5 Mechanisms regulating self-renewal and differentiation of neuronal progenitor cells

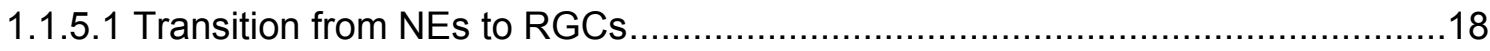

1.1.5.2 Symmetric and asymmetric cell division of neuronal progenitor cells ...............19

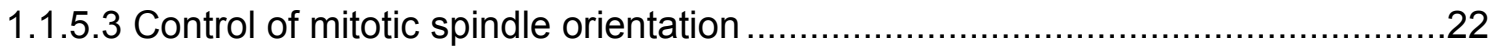

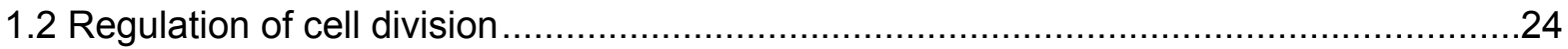

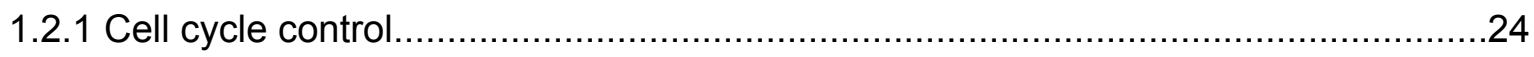

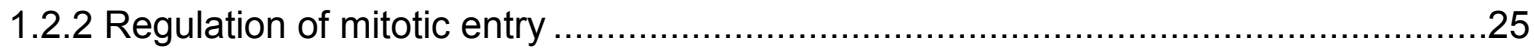

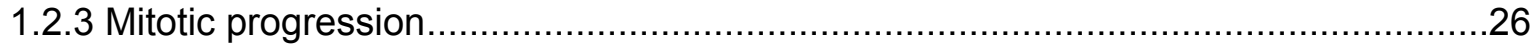

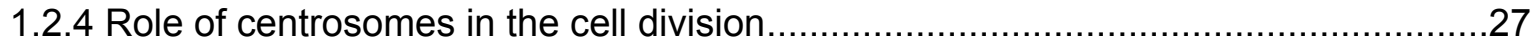

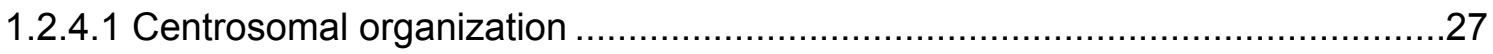

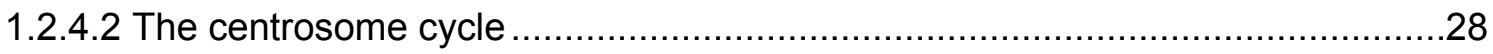

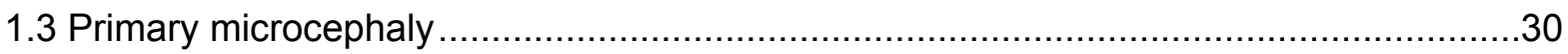

1.3.1 Clinical features of human primary microcephaly ……..........................................30

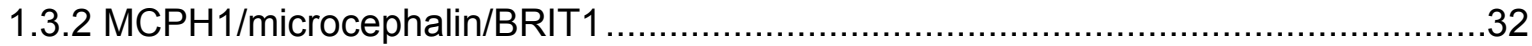

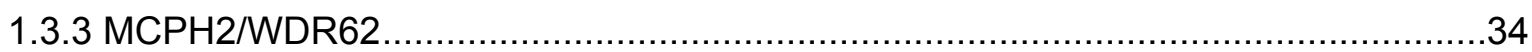




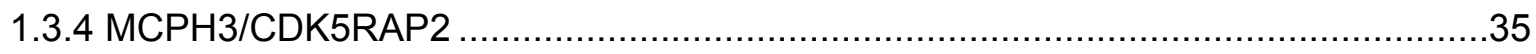

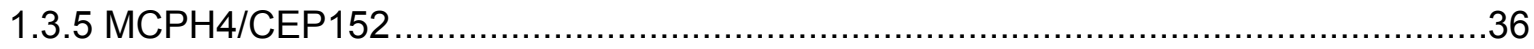

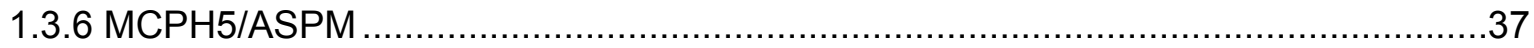

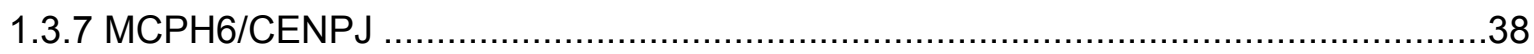

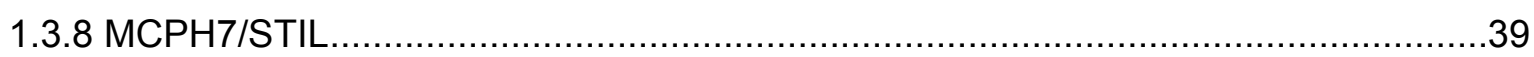

1.3.9 Common role of MCPH proteins in cortical development ...................................40

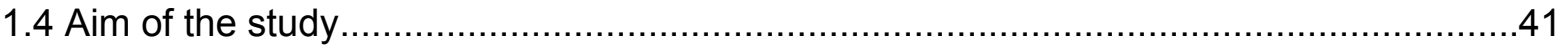

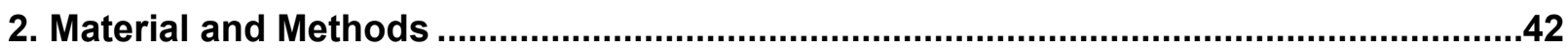

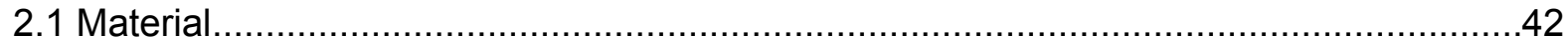

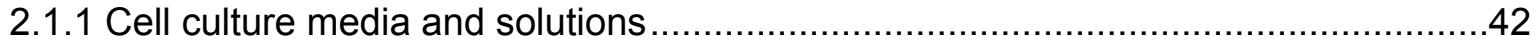

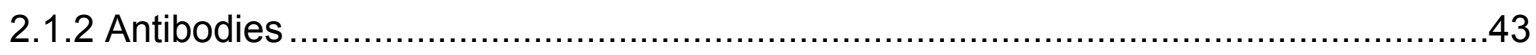

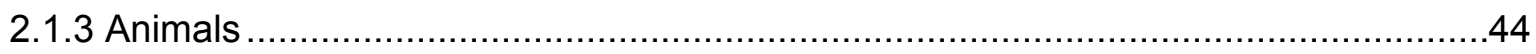

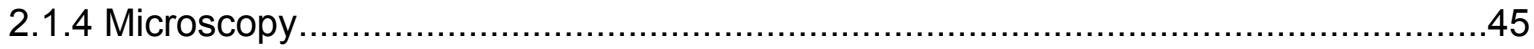

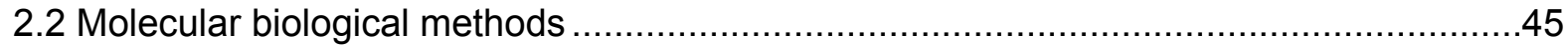

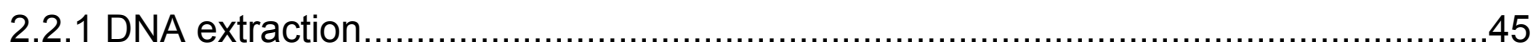

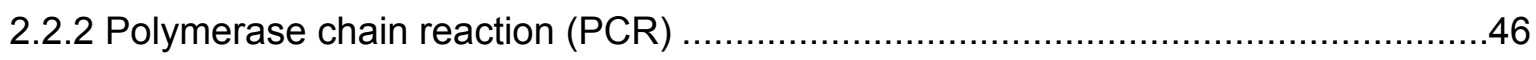

2.2.3 Construction of short hairpin RNA (shRNA)-vectors .............................................47

2.3 Mouse embryonic fibroblast (MEF) culture and methods .............................................48

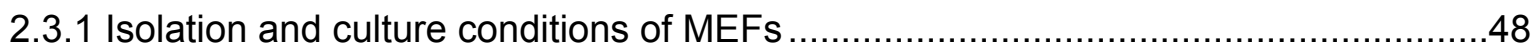

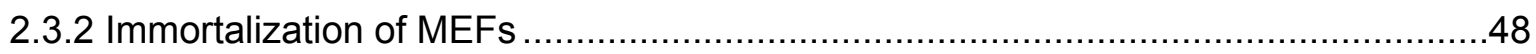

2.3.3 Transfection of immortalized MEFs ……..........................................................49

2.3.4 Immunocytochemistry of transfected MEFs.....................................................

2.4 Mouse embryonic neuronal progenitor cell culture and methods ..............................50

2.4.1 Isolation and culture conditions of neuronal progenitor cells ................................50

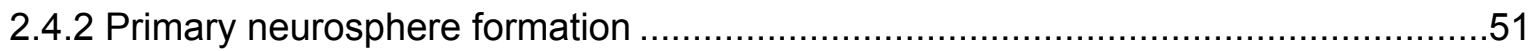

2.4.3 Determination of self-renewal capacity of neuronal progenitor cells ......................52

2.4.4 Immunocytochemistry of neuronal progenitor cells ..........................................52

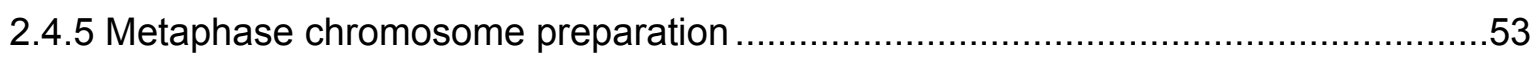

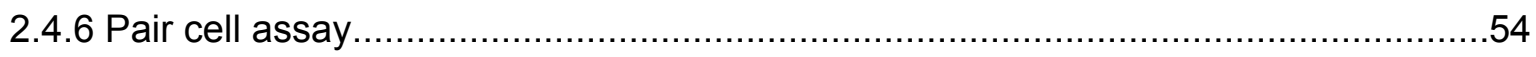

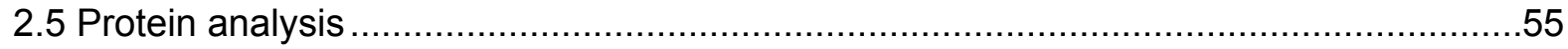

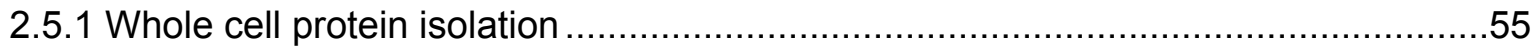




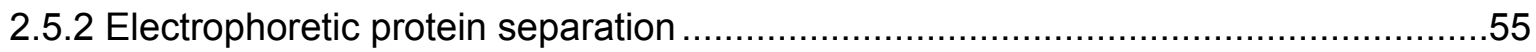

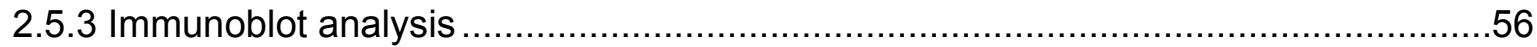

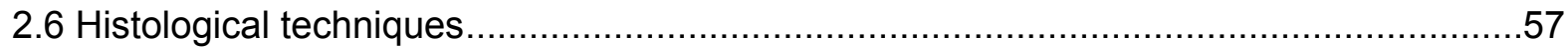

2.6.1 Isolation and fixation of embryonic and postnatal mouse brains .........................57

2.6.2 Preparation of paraffin embedded mouse brain sections .................................57

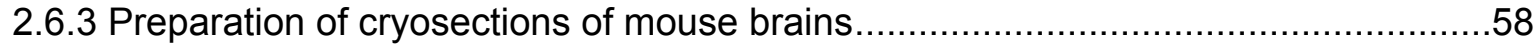

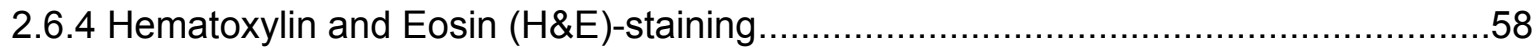

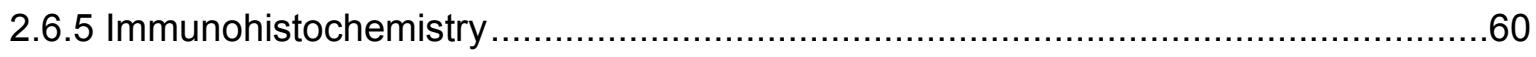

2.6.6 In vivo bromodeoxyuridine (BrdU)-proliferation assay ...................................60

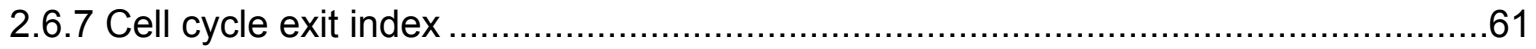

2.6.8 Terminal deoxynucleotidyl transferase dUTP nick end labeling (TUNEL) on mouse brain sections.

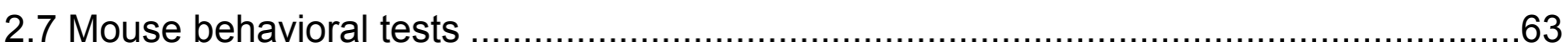

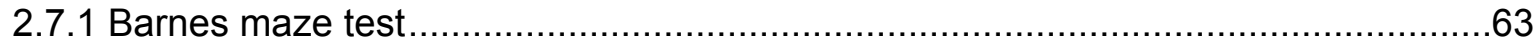

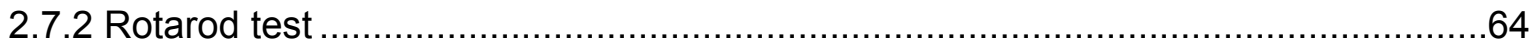

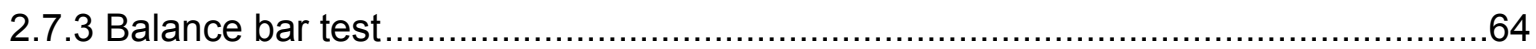

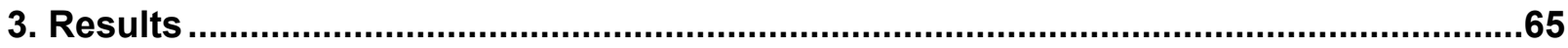

3.1 Deletion of Mcph1 in mice leads to primary microcephaly ......................................65

3.1.1 Reduced brain size and weight of $M c p h 1^{\Delta / \Delta}$ mice at newborn stage and adulthood 65

3.1.2 Reduced cerebral cortical thickness of $M c p h 1^{\Delta / \Delta}$ mice at P0 .............................67

3.1.3 Conditional CNS-specific deletion of Mcph1 leads to primary microcephaly ...........67

3.1.4 Mcph $1^{\Delta / \Delta}$ mice showed normal behaviour ................................................. 70

3.1.4.1 Barnes maze test showed normal learning and memory ability of Mcph1 /

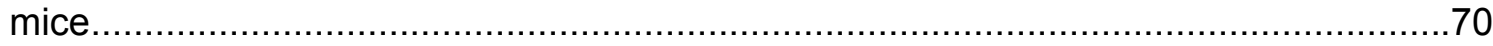

3.1.4.2 $\mathrm{Mcph}^{\Delta / \Delta}$ mice have normal motor coordination ......................................72

$3.2 \mathrm{Mcph}^{1 / \Delta}$ embryos have defects in neurogenesis.............................................

3.2.1 Reduced size of neuronal layer of $M c p h 1^{\Delta / \Delta}$ embryos ........................................73

3.2.2 Normal proliferation rate in $M c p h 1^{\Delta / \Delta}$ developing cortex at mid-phase of

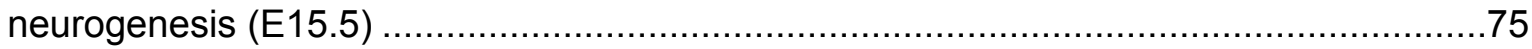


3.2.3 Reduced proliferation in the developing cortex of $M c p h 1^{\Delta / \Delta}$ embryos at the end of

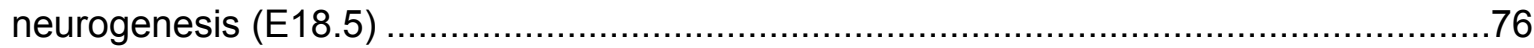

3.2.4 Reduced number of cycling cells in $M c p h 1^{\Delta / \Delta}$ embryos at E17.5 .........................78

3.2.5 Increased apoptosis in the VZ and SVZ of the telencephalon in Mcph ${ }^{\Delta / \Delta}$ embryos 79

3.2.6 No chromosomal aberrations of $M c p h 1^{\Delta / \Delta}$ neuronal progenitor cells

3.3 $M c p h 1^{\Delta / \Delta}$ neuronal progenitor cells show impaired self-renewal capacity in vitro .82

3.3.1 Normal proliferation of $M c p h 1^{\Delta / \Delta}$ E14.5 neuronal progenitor cells and PO CNSprogenitor cells in vitro

3.3.2 $M c p h 1^{\Delta / \Delta}$ neuronal progenitor cells show reduced self-renewal capacity in vitro......85 3.4 Mcph1 $1^{\Delta / \Delta}$ embryos show increased asymmetric progenitor cell division in the dorsal telencephalon .88

3.4.1 Mcph $1^{\Delta / \Delta}$ embryos show an increased cell cycle exit in the dorsal telencephalon....88

3.4.2 Analysis of the cleavage plane orientation of mitotic RGCs .90

3.4.3 Increased number of Mcph1-deficient mitotic RGCs bypassing the apical plasma membrane

3.4.4 Increased number of asymmetric cell divisions of Mcph1-deficient neuronal progenitor cells in vitro

3.5 Mcph1-deletion leads to premature mitosis of neuronal progenitor cells caused by centrosomal loss of Chk1

3.5.1 $\mathrm{Mcph}^{\Delta / \Delta}$ neuronal progenitor cells show reduced centrosomal localization of Chk1 in G2-phase

3.5.3 $\mathrm{Mcph} 1^{\mathrm{s} / \Delta}$ neuronal progenitor cells show hypercondensed metaphase chromosomes 101

3.6 Uncoupling of the cell cycle from the centrosome cycle results in spindle alignment defects and apoptosis 103

3.6.1 Mcph1 ${ }^{\Delta / \Delta}$ neuronal progenitor cells show premature mitotic entry 103

3.6.2 The centrosome cycle of $M c p h 1^{\Delta / \Delta}$ neuronal progenitor cells lags behind the mitotic progression 105

3.6.3 Mitotic progression with immature centrosome results in mitotic spindle defects of Mcph1-deficient neuronal progenitor cells 107

3.6.4 Mitotic spindle defects and mitotic catastrophe in the VZ of $M c p h 1^{\Delta / \Delta}$ embryos .....109 
3.6 Chk1 and MCPH1 function in the same pathway to regulate mitotic entry 111

3.6.1 Knockdown of Chk1 leads to aberrant mitotic spindles in MEFs 111

3.6.2 Knockdown of Cdc25b corrects mitotic spindle defects of Chk1 knockdown and $M c p h 1^{\Delta / \Delta}$ MEFs

4. Discussion. 117

4.1 Primary microcephaly of a MCPH1 mouse model. 117

4.2 $\mathrm{MCPH} 1$ prevents unscheduled asymmetric cell divisions 121

4.3 Deletion of Mcph1 affects centrosomal maturation 124

4.4 MCPH1 functions in the centrosomal Chk1-Cdc25B pathway to regulate mitotic entry 126

4.5 Cell death of Mcph1-deficient neuronal progenitor cells 128

4.6 Conclusions. 131

5. References. 134

6. Appendix. 157

6.1 Abbreviation list. 157

6.2 Acknowledgements 159

6.3 Curriculum Vitae 161 


\section{Abstract}

Primary Microcephaly (MCPH), an autosomal recessive disorder, is characterized by a reduction of the brain size in particular the cerebral cortex size. MCPH can be caused by mutations in any of seven MCPH loci: Microcephalin, WDR62, CDK5RAP2, CEP152, ASPM, CENPJ and STIL. All of the MCPH gene products have been shown to associate with the centrosomes either throughout the cell cycle or specific during mitosis. MCPH1, encoded by Microcephalin, has been shown to function in the DNA damage response and chromosome condensation. However, the role of $\mathrm{MCPH} 1$ in determining the mammalian brain size is not known.

Aim of the study was to identify the function of MCPH1 in the development of the central nervous system. We therefore disrupted the Mcph1 gene in mice. Mutant mice exhibit microcephaly, due to defects in the embryonic development of the cerebral cortex. Deletion of Mcph1 does not affect the proliferation rate of neuronal progenitor cells, but compromises their self-renewal capacity. In contrast, Mcph1 mutant embryos show a higher cell cycle exit of neuronal progenitor cells, suggesting a disturbed cell division mode. Indeed, we found that deletion of Mcph1 promotes neuronal progenitor cells to undergo neurogenic asymmetric cell division. Mcph1-deficient neuronal progenitor cells show a misregulation of the centrosomal localization of Chk1 in G2-phase of the cell cycle. Centrosomal Chk1 inhibits mitotic entry through temporary inhibition of Cdc25B and Cdk1. As a result, cells prematurely activate Cdk1 leading to early mitotic entry thereby uncoupling the cell cycle from the centrosome cycle. The uncoupling of these two cycles results in mitotic spindle defects and apoptotic cells, due to mitotic failure. Moreover, defects of the mitotic spindle alignment affects the orientation of the cleavage plane of dividing neuronal progenitor cells, thereby promoting neurogenic asymmetric divisions.

We conclude that MCPH1 regulates the type of neuronal progenitor division mode by ensuring proper mitotic entry through the centrosomal Chk1-Cdc25B-pathway. Hence, MCPH1 is important for the amplification of the neuronal progenitor pool and consequently a sufficient neuron production during embryonic neurogenesis to determine the brain size. 


\section{Zusammenfassung}

Primäre Mikrozephalie (MCPH) ist eine autosomal-rezessive Krankheit, die charakterisiert ist durch eine kleine Gehirngröße, wobei der zerebrale Kortex am stärksten betroffen ist. MCPH wird verursacht durch Mutationen in einem von sieben MCPH Genen: Microcephalin, WDR62, CDK5RAP2, CEP152, ASPM, CENPJ und STIL. Alle MCPH Genprodukte interagieren mit den Zentrosomen, entweder während des gesamten Zellzyklus oder spezifisch während der Mitose. Darüber hinaus wurde für MCPH1, das von Microcephalin kodiert wird, eine Funktion in der DNA-Schadensantwort und Chromosomenkondensation gezeigt. Es ist jedoch nicht bekannt, welche Funktion von MCPH1 in der Gehirnentwicklung eine wichtige Rolle spielt.

Ziel dieser Studie war es die Funktion von MCPH1 in der Entwicklung des zentralen Nervensystems zu untersuchen. Es wurden daher knockout-Mäuse von Mcph1 generiert. Die Mcph1-mutierten Mäuse zeigen Mikrozephalie aufgrund fehlerhafter embryonaler Entwicklung des zerebralen Kortex. Die Deletion von Mcph1 hat keinen Einfluss auf die Proliferation von neuronalen Vorläuferzellen, aber beeinträchtigt ihre Selbsterneuerungskapazität. Die Mcph1mutanten neuronalen Vorläuferzellen zeigen einen erhöhten Zellzyklusaustritt, welches eine gestörte Zellteilungsart andeutet. Tatsächlich konnte gezeigt werden, dass eine Deletion von Mcph1 neuronale Vorläuferzellen zur neurogenen asymmetrischen Zellteilung treibt. Mcph1defiziente neuronal Vorläuferzellen zeigen eine verminderte zentrosomale Lokalization von Chk1 in der G2-phase des Zellzyklus. Zentrosomal lokalisiertes Chk1 verhindert einen Eintritt in die Mitose durch temporäre negative Regulation von Cdc25B und Cdk1. Mcph1-defiziente Zellen aktivieren frühzeitig Cdk1 und leiten einen vorzeitigen Eintritt in die Mitose ein, was zu einer Entkopplung des Zellzyklus vom zentrosomalen Zyklus zur Folge hat. Diese Entkopplung führt zu Defekten der mitotischen Spindeln und Apoptose aufgrund von mitotischen Versagens. Darüber hinaus können Defekte in der Ausrichtung der mitotischen Spindeln die Zellteilungsebene von neuronalen Vorläuferzellen beeinflussen, was zu einer Neigung zur neurogenen asymmetrischen Zellteilung führt.

Zusammenfassend kann gesagt werden, dass $\mathrm{MCPH} 1$ die Zellteilungsart neuronaler Vorläuferzellen durch Regulation des zentrosomalen Chk1-Cdc25B-Signalweges zum mitotischen Eintritt beeinflusst. MCPH1 spielt eine wichtige Rolle zur Bestimmung der Anzahl neuronale Vorläuferzellen und zur ausreichenden Neuronenproduktion während der embryonalen Neurogenese. 


\section{Introduction}

The human brain and its development have been the focus of research since many years. In the evolutionary lineage to humans, the most notable trend is the enlargement of the brain, especially of the cerebral cortex. The human cerebral cortex is the region of the brain that is predominantly involved in higher cognitive functions and is important for perception, memories, thoughts and language. There is a correlation between the encephalization quotient (ratio of brain to body weight) and cognitive complexity (Williams, 2002). It has been suggested that genes involved in the development of the cerebral cortex are likely to play also a role in the evolution of the brain (Williams, 2002). Elucidating the mechanisms of cortical development therefore may provide a better understanding of its functional capacities.

\subsection{Development of the cerebral cortex}

\subsubsection{Early events in neural tube development}

The early events of central nervous system (CNS) development are highly conserved among vertebrates. The first step in CNS development is the neural induction of the ectoderm, one of the three main cell layers that also include mesoderm and endoderm (for review see Kandel et al., 2000). In vertebrates, the neural induction occurs during early gastrulation with the expression of the genetic program that distinguishes the nervous system from non-neuronal tissues. This results in the formation of a pseudostratified neuroepithelium from the ectoderm, known as the neural plate that gives rise to the entire CNS. The organization of the neural plate is highly conserved among vertebrates (Rubenstein et al., 1998). Soon after the neural plate has formed it begins to lengthen and fold leading to closure of the neural tube (Figure 1; reviewed in Greene and Copp, 2009). 
A
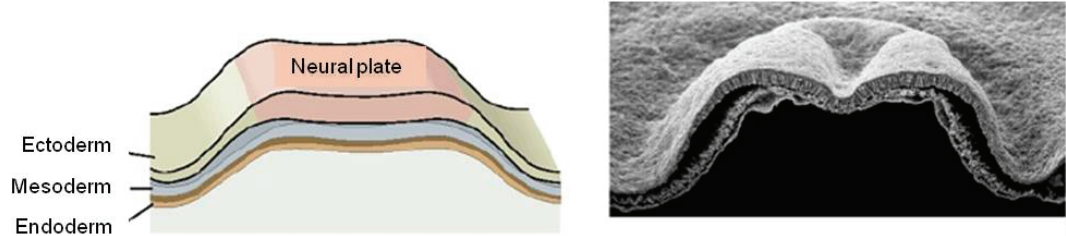

B
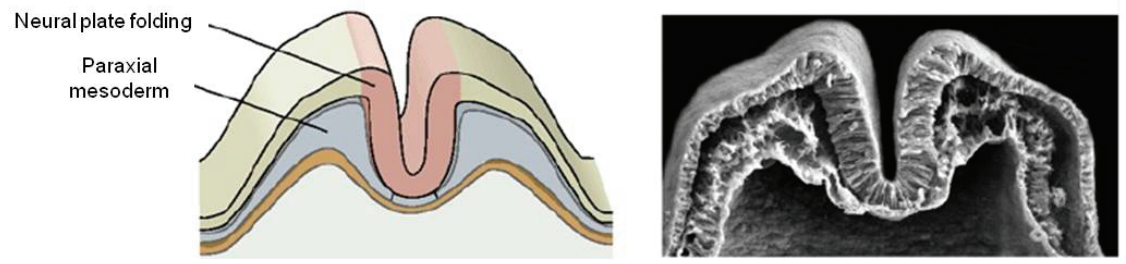

C
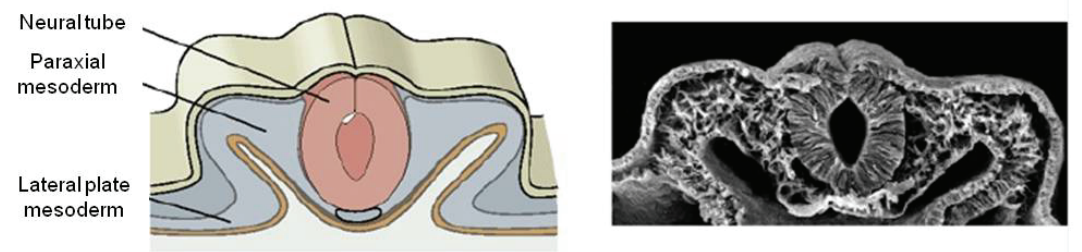

Figure 1: Closure of the neural tube.

Schematic view and scanning electron micrographs of chicken embryos show the closure of the neural tube as early step in the CNS development that is highly conserved among vertebrates.

(A) The neural plate derives from the ectoderm.

(B) Folding of the neural plate. The paraxial mesoderm derives from the mesoderm and gives rise to notochord and somites.

(C) Closure of the neural plate to form the neural tube that gives rise to the whole CNS of vertebrates (modified from Kandel et al., 2000).

The neural tube consists of rapidly dividing neuroepithelial cells. However, the proliferation is not uniform along the neural tube leading to an expansion at different rates resulting in the formation of different structures. The caudal region of the neural tube becomes the spinal cord, whereas the rostral region of the neural tube gives rise to three brain vesicles: the prosencephalon (forebrain), the mesencephalon (midbrain) and the rhombencephalon (hindbrain; Figure 2). Later in development, the prosencephalon divides into the telencephalon and the diencephalon. The mesencephalon divides into the metencephalon and 
myelencephalon thereby leading to the development of the neural tube from the three-vesiclestage to a five-vesicle-stage (Figure 2; Kandel et al., 2000).
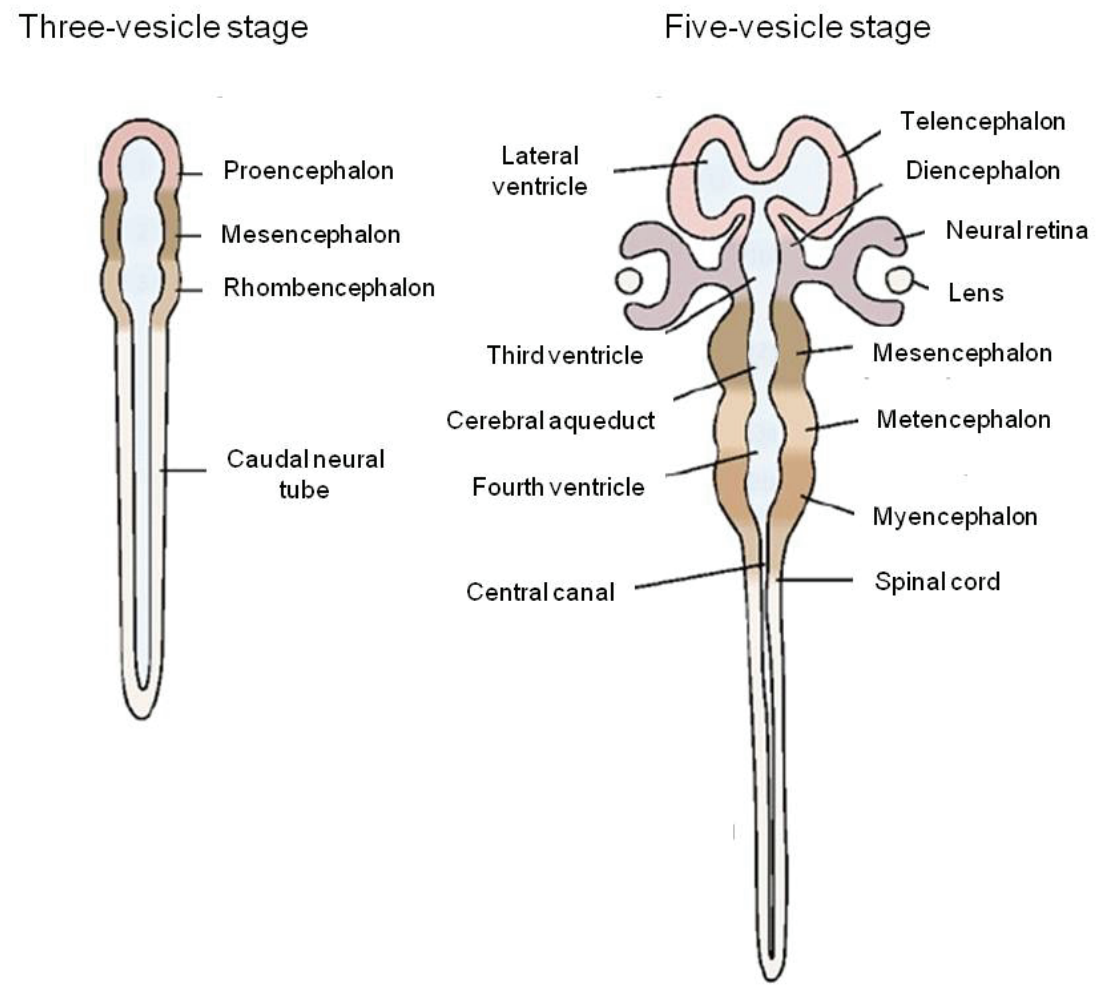

Figure 2: Development of the neural tube.

Schematic view of neural tube development from three-vesicle-stage to five-vesicle-stage of vertebrates (modified from Kandel et al., 2000).

\subsubsection{Morphology of the developing forebrain}

The developing forebrain comprises the telencephalon, which will give rise to the cerebral cortex, the basal ganglia and the olfactory bulb, and the diencephalon that gives rise to the hypothalamus and the thalamus. During the second half of embryogenesis (from E11 in mouse), different progenitor zones in the telencephalon are apparent (Figure 3). At the ventral side of the telencephalon, the lateral ganglionic eminence and the medial ganglionic eminence 
are found, which give rise to the striatum and the pallidum, respectively. Both, the striatum and the pallidum comprise the basal ganglia. The dorsal part of the telencephalon comprises the pallium, in which radial migrating glutamergic cortical neurons of the cerebral cortex are generated. However, in the lateral and medial ganglionic eminences $\gamma$-aminobutyric-acidreleasing (GABAergic) interneurons are generated (Nieuwenhuys et al., 2007), which migrate tangentially to the cerebral cortex (Figure 3). Telencephalic neuronal progenitor cells have unique gene expression profiles, depending on their location. For example, the transcription factors Pax6 and Emx1 are expressed in the proliferative area of the dorsal telencephalon (Boncinelli et al., 1993; Shimamura et al., 1997), whereas Msh2 and Gsh2 are expressed in both ganglionic eminences (Corbin et al., 2003; Rallu et al., 2002) and Nkx2.1 is expressed specific in the medial ganglionic eminence (Ericson et al., 1995).

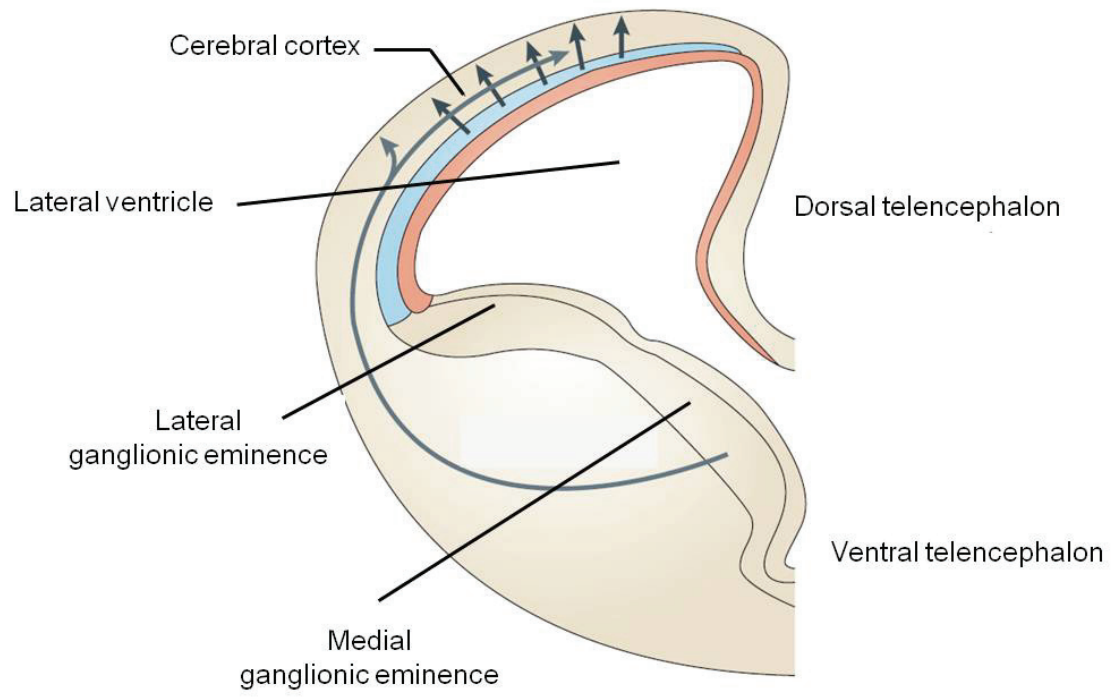

Figure 3: Morphology of the embryonic forebrain.

Schematic view of one hemisphere of a coronal mouse embryonic forebrain section at E13.5. Glutamergic cortical neurons generated in the ventricular zone (red area) and subventricular zone (blue area) migrate radially to the cerebral cortex (black arrows). GABAergic interneurons generated in the lateral and medial ganglionic eminence migrate tangentially to the cerebral cortex (modified from Dehay and Kennedy, 2007). 


\subsubsection{Neuronal stem and progenitor cells of the dorsal telencephalon}

At early developmental stages the dorsal telencephalon exists as a pseudostratified neuroepithelium that is occupied by the neuronal stem cells: the neuroepithelial cells (NEs). NEs are bipolar cells contacting the apical (ventricular) surface as well as the basal surface of the neuroepithelium (Figure 4). The pseudostratification is a result of a process called interkinetic nuclear migration. The nuclei of NEs migrate along the apical-basal axis depending on the cell cycle phase. The nuclei migrate to the basal side in G1-phase and undergo Sphase at the basal side. In G2-phase the nuclei migrate to the apical side and mitosis occurs at the apical surface (Sauer, 1935; Takahashi et al., 1993). The neuroepithelial cells divide rapidly to expand their pool until around E10 in mouse, the time when cortical neurogenesis begins through switching the cell division mode from symmetric proliferative cell divisions to asymmetric divisions to produce a daughter cell that is restricted to the neuronal lineage (Noctor et al., 2004; Noctor et al., 2007). At this stage NEs, downregulate certain epithelial properties, such as tight junctions (Aaku-Saraste et al., 1996) and in addition acquire properties of glial cells and therefore transform into the related but distinct cell type radial glial cells (RGCs; Figure 4).

RGCs, in contrast to NEs, express astroglial markers such as astrocyte-specific glutamate transporter (GLAST) and glial fibrillary acidic protein (GFAP; reviewed in Campbell and Gotz, 2002) and show additional properties of astroglial cells, like glycogen granules (Gadisseux and Evrard, 1985). However, RGCs maintain certain NE properties, such as nestin gene (Hartfuss et al., 2001) and apical localization of centrosomes (Chenn et al., 1998). Importantly, RGCs maintain the apical-basal polarity and interkinetic nuclear migration. However, migration of the nuclei of RGCs does not span the entire cytoplasm as in NEs. The nuclei of RGCs migrate along the apical surface and basal side of the ventricular zone (VZ), a defined region next to the ventricle, in a similar cell cycle dependent manner as the nuclei of NEs, thereby maintaining a pseudostratified epithelium in the VZ. However, the cytoplasm of RGCs spans the entire cortical wall from the apical surface to the pial surface (Noctor et al., 2001; Rakic, 1972). This RGC morphology is critical for the migration of neurons, since neurons generated in the VZ use these RGC processes as a guide to migrate radially to the cortical plate (CP) that is the neuronal layer of the embryonic dorsal telencephalon (Noctor et al., 2001; Rakic, 1972). At the end of mammalian neurogenesis, most RGCs transform into astrocytes 
(reviewed in Mission et al., 1991) and a subpopulation of RGCs generates oligodendrocytes (Kessaris et al., 2006). Therefore, RGCs are multipotent progenitor cells in the dorsal telencephalon that generate neurons, astrocytes and oligodendrocytes.

During neurogenesis, RGCs divide asymmetrically to produce one daughter RGC and one postmitotic neuronal daughter cell (lacopetti et al., 1999). However, after the onset of neurogenesis, RGCs are also able to produce another neuronal progenitor cell type: the intermediate progenitor cells (IPCs) also known as basal progenitors (Miyata et al., 2004; Noctor et al., 2004). IPCs lack the contact to the apical surface and to the pial surface (Miyata et al., 2004), therefore losing the apical-basal polarity and also differ from RGCs in the gene expression profile. For example, the transcription factors Pax6 and Tbr2 are specific markers for RGCs and IPCs, respectively (Englund et al., 2005). During neurogenesis IPCs form another proliferative layer in the dorsal telencephalon: the subventricular zone (SVZ) that is at the basal side of the VZ (Figure 4). After generation, most IPCs undergo only one round of cell division in the SVZ to produce two daughter neurons by symmetric neurogenic cell division (Haubensak et al., 2004). Hence, IPCs function to increase the number of neurons by one additional round of cell division and there is a correlation between the size of the SVZ and the number of neurons in the cerebral cortex of different species. For example, in contrast to rodents, the primate brains have an additional layer, the outer SVZ (Smart et al., 2002). The outer SVZ contains proliferative cells, presumably a subtype of IPCs that still show properties of RGCs (Fietz et al., 2010). The outer SVZ may contribute to the massive expansion of the cerebral cortex in primates.

Another cell type can be found in the VZ of the dorsal telencephalon, the short neural precursors (SNPs), which have contact to the apical surface and a basal process of variable length that retracts during mitosis. The retraction of the basal process does not occur in RGCs during mitosis. SNPs are unique among the neuronal progenitor cell in the telencephalon in the expression of the tubulin $\alpha-1$ promoter (Gal et al., 2006). Thus, the dorsal telencephalon contains multiple types of neuronal progenitor cells. 


\subsubsection{Corticogenesis}

\subsubsection{Cortical neurogenesis}

The basic principles of corticogenesis have been described in the Boulder Committee with standarized nomenclature (Boulder-Commitee, 1970). At the time when neurogenesis begins, around E10 in mice, NEs transform into RGCs that divide asymmetrically to produce one daughter cell restricted to the neuronal lineage (Figure 4). As neurogenesis proceeds, neurons are generated by symmetric cell divisions of IPCs in the SVZ, in addition to the neuron production in the VZ by asymmetrically dividing RGCs. The postmitotic neurons migrate radially from their birth place, the VZ or the SVZ along the basal processes of RGCs to the basal side of the neurepithelium (Noctor et al., 2001; Rakic, 1972), to form the preplate $(\mathrm{PP})$. Later, the PP is subdivided into the outer marginal zone (MZ) and the inner layer subplate (SP). In the MZ, Cajal-Retzius cells are located, which produce the extracellular matrix protein Reelin that is important for neuronal migration and formation of the cortical layers through stabilizing functions (Frotscher, 2010). The neurons continue the migration into radial direction until they reach the $\mathrm{MZ}$, leading to the formation of the neuronal layer $\mathrm{CP}$ between the $\mathrm{MZ}$ and SP. Neurons generated later during neurogenesis migrate through the SP and the CP to form different neuronal layers of the CP (Figure 4). The formation of the different cortical layers occurs in an inside-out-mechanism, which means that neurons generated earlier form the inner cortical layers and later born neurons migrate through the inner layers to form the more superficial layer (Angevine and Sidman, 1961). At the end of neurogenesis, the CP consists of six layers which persist throughout adulthood. However, in addition to the radial migrating glutamergic neurons generated in VZ and SVZ of the dorsal telencephalon, neurons born in the lateral and medial ganglionic eminences of the ventral telencephalon, migrate tangentially into the cortical plate (Figure 3). These tangential migrating neurons of the ventral telencephalon are mainly inhibitory interneurons that use GABA as neurotransmitter (Anderson et al., 1997). 


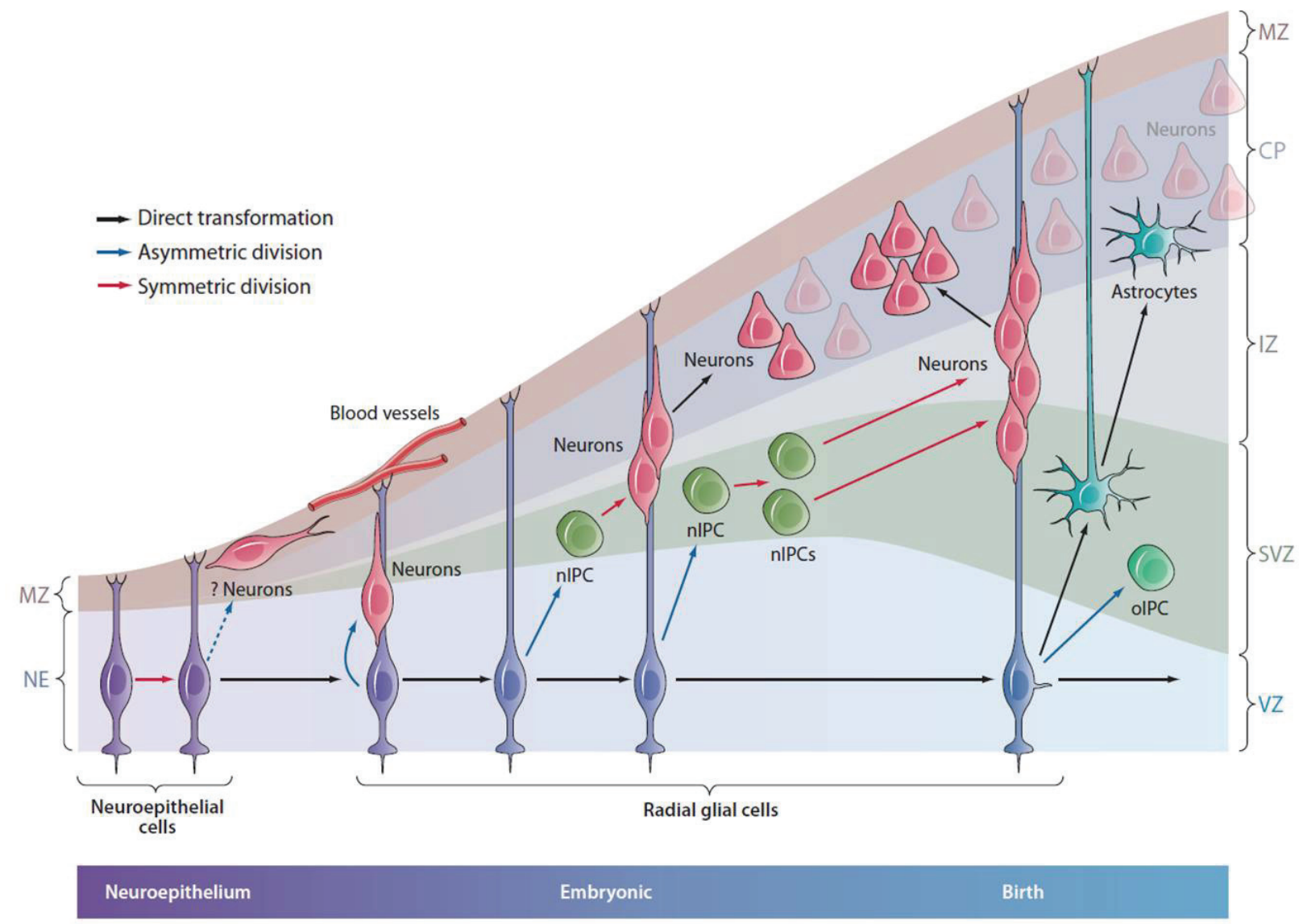

Figure 4: Corticogenesis.

The model shows the development of the cerebral cortex. Neuroepithelial cells divide symmetrically in the neuroepithelium (NE) to expand and transform into radial glial cells at the onset of neurogenesis. Radial glial cells reside in the ventricular zone (VZ) and divide asymmetrically to generate neurons and neurogenic intermediate progenitor cells (nIPC) that populate the subventricular zone (SVZ). nIPCs divide symmetrically to generate neurons. Neurons generated in the dorsal telencephalon migrate radially through the intermediate zone (IZ) to the marginal zone (MZ) and reside in the cortical plate (CP). At the end of neurogenesis, radial glial cells transform into astrocytes or intermediate progenitor cells that produce oligodendrocytes (oIPC). Not depicted in this model: interneurons generated in the ventral telencephalon migrate tangentially to the cerebral cortex (modified from Kriegstein and AlvarezBuylla, 2009).

\subsubsection{Gliogenic phase of cortical development}

The production of glial cells starts during late embryonic development (Figure 4) and continues postnatally. RGCs are multipotent progenitor cells, since not only neurons are 
derived directly or indirectly form this cell type, but also astrocytes and oligodendrocytes. At the end of neurogenesis, RGCs lose their ventricular attachment, migrate toward the CP and transform into astrocytes. Some of the astrocytes that transformed from RGCs divide postnatally before terminal differentiation and therefore represent astrocytic progenitor cells (reviewed in Mission et al., 1991). The pattern of RGCs to generate neurons first and subsequently astrocytes is also observed of cultured single RGCs in vitro (Qian et al., 2000; Shen et al., 2006).

A subpopulation of RGCs remains attached at the ventricular surface and generates oligodendrocytes postnatally (Kessaris et al., 2006). However, in many vertrabrate species, some ventricular attached RGCs persist postnatally in the SVZ of adult brains (Figure 4). Although, these cells are morphological different to RGC, since they retract their basal process, they have a similar function in adult brains. They serve as a type of neuronal progenitor cells for the adult neurogenesis in the SVZ and in addition are able to generate glial cells (see Gould, 2007 for review on adult neurogenesis).

\subsubsection{Mechanisms regulating self-renewal and differentiation of neuronal progenitor cells}

\subsubsection{Transition from NEs to RGCs}

With the transition from NEs to RGCs the cell division mode switches from symmetric selfrenewal divisions to asymmetric cell divisions that produce differentiated cells (Figure 4). The timing of onset of this switch affects the final neuron number, since the symmetric cell division mode of NEs leads to an expansion of the pool of neuronal progenitor cells. For example the neurogenic phase in humans starts later than in non-human primates leading to several extra rounds of cell division, resulting in a big increase in cerebral cortex size (Rakic, 1995). The transition from NEs to RGCs is regulated at least in part by the fibroblast growth factor (Fgf) signaling, since overexpression of an active Fgf receptor (Fgfr2) in vivo promotes differentiation into RGCs in mouse (Yoon et al., 2004). Furthermore, deletion of the Fgf ligand Fgf10 in mouse leads to a delay in RGC appearance and reduced asymmetric cell divisions 
resulting in an increased neuron number and bigger size of the cerebral cortex (Sahara and O'Leary, 2009). In addition, the Notch signaling is involved in the NE to RGC transition. Overexpression of an active form of Notch1 (Notch1 intracellular domain, NIC) before the onset of neurogenesis promotes the differentiation into RGCs (Gaiano et al., 2000). Mice deficient for both Notch1 and Notch3 in the forebrain show a reduced gene expression of Blbp, encoding for brain lipid-binding protein that is required for RGC morphology (Anthony et al., 2005). A recent study suggests that extrinsic signals might be an additional mechanism for the onset of neurogenesis. The study showed, that retinoic acid, secreted by meninges in the dorsal telencephalon is an important factor in determining the start of neurogenesis (Siegenthaler et al., 2009). Later during neurogenesis extrinsic signals are also important to promote the switch of RGCs from neurogenesis to gliogenesis. Newly generated neurons send signals back to the RGCs to promote the gliogenesis. Upregulation of Fgf9 and Ntf3 in mice is sufficient to promote the transition of RGCs to astrocytes (Seuntjens et al., 2009).

\subsubsection{Symmetric and asymmetric cell division of neuronal progenitor cells}

Before the onset of neurogenesis, NEs undergo exclusively symmetric cell divisions to expand the pool of neuronal progenitor cell. RGCs instead are able to undergo two distinct types of asymmetric cell divisions. One type is the direct neurogenic division, by which RGCs produce one daughter neuron and one daughter RGC. Another type of asymmetric cell division is the indirect neurogenic division that generates one daughter RGC and one daughter IPC that produces two daughter neurons by symmetric division in the next round of cell division. However, RGCs are also able to undergo self-renewal symmetric cell divisions to produce two daughter RGCs (Noctor et al., 2001; Noctor et al., 2004).

The mechanisms controlling the cell division mode of neuronal progenitor cell are well studied in Drosophila melanogaster and Caenorhabditis elegans and seem to be conserved among vertebrates (for review see Knoblich, 2008). In mammals, the apical-basal polarity of NEs and RGCs is important for the symmetric and asymmetric division. For example loss of the epithelial cell polarity by disruption of the $L g / 1$ gene in mice leads to hyperproliferation of NEs and RGCs and reduced differentiation (Klezovitch et al., 2004). It has been proposed that the 
orientation of the cleavage plane is a determinant for the neuronal progenitor cell division mode, similarly to proliferative and neurogenic divisions of neuroblasts which are the Drosophila melanogaster neuronal progenitor cells. Cleavage planes that are orientated perpendicular to the ventricular surface (vertical divisions) result in self-renewal symmetric divisions, since distinct apical and basal cellular components would be equally distributed to the daughter cells. Whereas, an orientation of the cleavage plane horizontal to the ventricular surface (horizontal division) leads to asymmetric cell division because apical cellular components would be inherited by only one daughter cell and the basal components by the other daughter cell (Figure 5; for review see Gotz and Huttner, 2005; Zhong and Chia, 2008). It has been shown that in the developing CNS of Drosophila melanogaster the plane of division of neuroblasts can indeed predict the cell division mode (Fuerstenberg et al., 1998). In mammals however, horizontal divisions of RGCs are rare events (Landrieu and Goffinet, 1979; Smart, 1973). Importantly, the morphology of RGCs in mammals is different to neuroblasts in Drosophila melanogaster. RGCs are very elongated cells with apical-basal polarity and therefore the apical plasma membrane constitutes a very small fraction of the total plasma membrane (about 1 - 2\%; Kosodo et al., 2004). Dividing RGCs with a cleavage plane orientated vertically relative to the ventricular surface would then lead either to an equal inheritance of the apical plasma membrane to both daughter cells or to a bypass of the plasma membrane leading to the inheritance to only one daughter cell (Figure 5; Kosodo et al., 2004). One example of the apical components localized at the apical plasma membrane of RGCs is the Par-complex. The Par complex consists of the proteins Par-3, Par-6, PKC $\zeta$ and the small GTPase Cdc42 and is required for epithelial cell polarity (Joberty et al., 2000; Lin et al., 2000). The role of the Par-complex in establishing a cell polarity is through promoting the epithelial cell specific development of adherens junctions and tight junctions (Suzuki and Ohno, 2006). Additionally, aPKC, the Drosophila melanogaster homologue of $\mathrm{PKC} \zeta$, phosphorylates the protein Numb (Smith et al., 2007a), which was originally identified in Drosophila melanogaster as a cell fate determinant protein that segregates to the basal side of asymmetrically dividing neuroblasts (Betschinger and Knoblich, 2004). Numb promotes neuronal cell fate by inhibiting Notch signaling in a tissue-specific manner, through complex formation of Eps15 and AP-2, which leads to endocytosis of Notch (Berdnik et al., 2002; Smith et al., 2004). Notch signaling has a well established role in maintaining progenitor cell identity (Gaiano and Fishell, 2002). In mammals, Numb has to two homologues: Numb and Numb-like (Numbl), which have essential 
and redundant roles in mammalian brains (Li et al., 2003; Petersen et al., 2004). However, unlike in Drosophila melanogaster neuroblasts, mammalian Numb and Numbl seem not to localize to the basal side of RGC, but rather interact with adherens juctions at the apical endfeet of RGC together with a cadherin-catenin complex. It has been proposed that Numb promotes RGC polarity and adhesion by stabilizing adherens junctions (Rasin et al., 2007), therefore the function in vertebrates seem to be different to the function of Drosophila melanogaster Numb.

Symmetric division
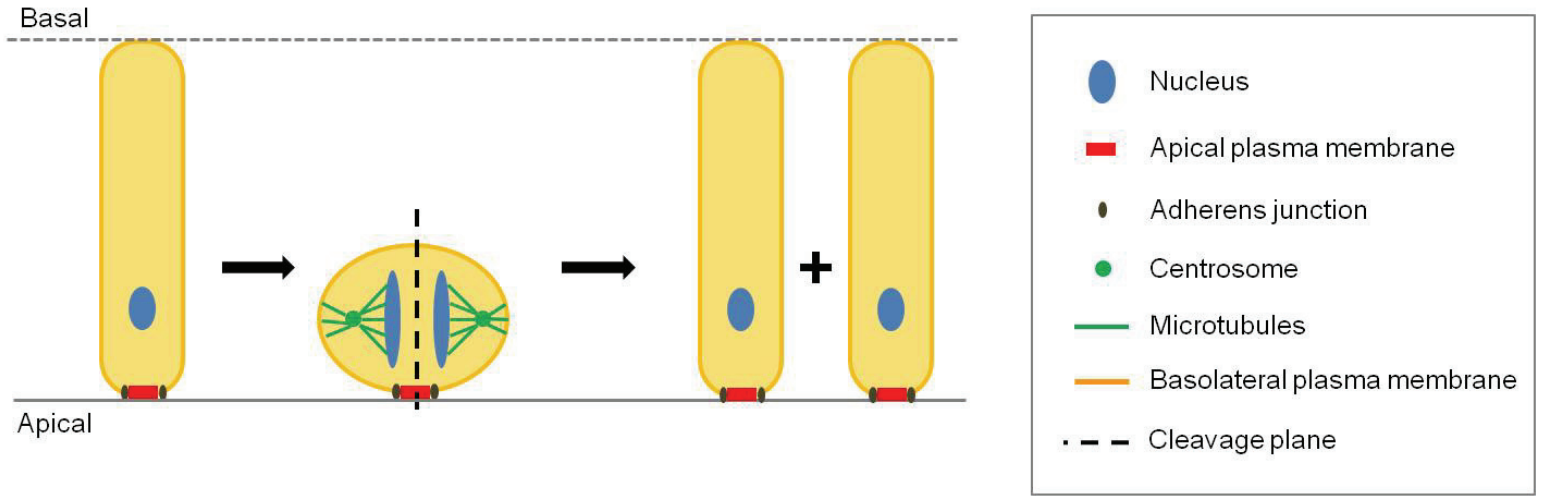

Asymmetric division

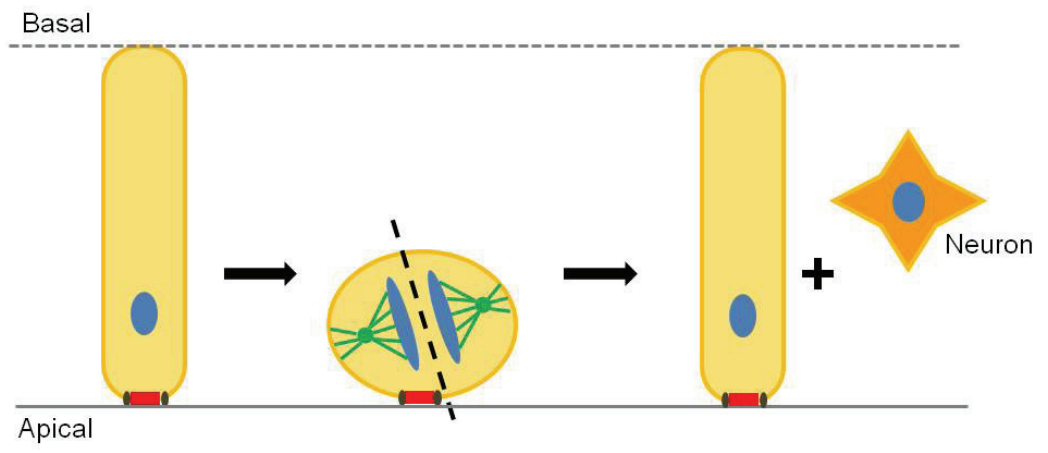

Figure 5: Symmetric and asymmetric cell division of RGCs.

The schematic model shows symmetric and asymmetric cell division mode of RGCs in the dorsal telencephalon. Equal inheritance of the apical plasma membrane results in symmetric division and unequal inheritance leads to asymmetric neurogenic cell division (modiefied from Gotz and Huttner, 2005). 
Dividing RGCs in which the cleavage plane bypasses the apical plasma membrane lead to the inheritance of the Par-complex to only one daughter cell, presumably leading to inhibition of the Notch signaling in the other daughter cells thereby inducing a neuronal cell fate (Figure 5). Additionally, bypass of the apical plasma membrane leads to the inheritance of adherens junctions and adhesion molecules to one daughter cell whereas the other daughter would lose its apical attachment (Marthiens and ffrench-Constant, 2009). Therefore, the VZ itself provides signals required for stem cell maintenance and was proposed to be termed as the stem cell niche (Marthiens et al., 2010).

\subsubsection{Control of mitotic spindle orientation}

Considering that the apical plasma membrane of RGCs constitutes only a very small fraction of the total plasma membrane (Kosodo et al., 2004), the mechanisms controlling the orientation of the cleavage plane have to operate very precisely. One of such mechanism is the control of mitotic spindle orientation, which affects the cleavage plane orientation relative to the apical surface and therefore has to be tightly controlled (Figure 5). Two main mechanisms seem to regulate the spindle orientation: the heterotrimeric G-protein function and functions of centrosomal proteins.

Studies of Drosophila melanogaster neuroblasts revealed that the heterotrimeric G-proteins function in the positioning of the mitotic spindles to affect the cell division mode. In neuroblasts, the protein Inscuteable, which is a binding partner of the apical plasma membrane protein Par-3, recruits the protein Pins to the apical cell cortex (Schober et al., 1999). Pins contains three GoLoco domains that are able to bind to the heterotrimeric Gprotein subunit Gai leading to activation of Pins (Nipper et al., 2007). Consequently, active Pins binds to the protein Mud, which is the Drosophila melanogaster homolog of the microtubule and dynein-binding protein NuMa. Mud then is able to capture astral microtubules from one of the spindle poles and orient the mitotic spindle (Bowman et al., 2006; Izumi et al., 2006; Siller et al., 2006). In vertrebrates, a similar role of heterotrimeric G-proteins in the alignment of the mitotic spindles of NEs and RGCs was reported (Sanada and Tsai, 2005). In the study of Sanada and Tsai, it was shown that keeping G $\beta \gamma$ subunits in a complex by using 
an inhibitor, results in changes of the cleavage plane orientation of RGCs to more vertical divisions. Addtionally, the mammalian Pins homologue, AGS3 regulates the spindle orientation in the developing mouse cortex (Sanada and Tsai, 2005). However, in mammals exists another Pins homologue, called LGN which was shown to bind to the microtubule binding protein NuMa and thereby regulates mitotic spindle organization in cell culture (Du et al., 2001). LGN itself is recruited by Gai subunit to the cell cortex (Du and Macara, 2004). Among the two Pins homologues, only LGN is localized asymmetrically in human mitotic neuronal progenitor cells (Fuja et al., 2004). Additional mechanistic insights came from a study of Nguyen-Ngoc et al, in which they show that the LGN-Gai complex together with other complex members, the GoLoco domain-proteins GPR-1 and GPR-2, affects the dynein-dependent microtubule pulling force through interaction with Lis-1, a component of the dynein complex (Nguyen-Ngoc et al., 2007). Although this study is based on work on Caenorhabditis elegans, the mechanism might be conserved, since Lis-1 was shown to be essential for precise mitotic spindle orientation of NEs in mouse (Yingling et al., 2008). However, the mechanisms of how modulating dynein-dependent microtubule pulling force affect the mitotic spindle orientation of mammalian NEs and RGCs and thereby the cell division mode, remain to be determined.

Another mechanism, by which the orientation of cleavage plane is controlled, is governed by centrosomal proteins. Certain centrosomal proteins seem to regulate the positioning of the mitotic spindle poles, which subsequently set the orientation of the cleavage plane (Figure 5). Examples are the proteins encoded by the so called microcephaly genes, which cause the human disease primary microcephaly when mutated (see chapter 1.3). Interestingly, most of these genes are highly expressed in the VZ of the embryonic brains and all of the encoded proteins seem to interact with the centrosomes either throughout the cell cycle or specific during mitosis. These proteins do not appear to be required for cell division, but rather are required for the balance of symmetric versus asymmetric cell division of neuronal progenitor cells by positioning the mitotic spindle poles. The microcephaly genes will be discussed in detail in chapter 1.3 . 


\subsection{Regulation of cell division}

\subsubsection{Cell cycle control}

The cell cycle is the period from one cell division to another and is divided into four phases, G1, S, G2 and mitosis (M-phase). In G1-phase the cell prepares for DNA replication which occurs during S-phase. In G2-phase the cell with duplicated DNA prepares for cell division in mitosis. Mitosis itself is divided into prophase, prometaphase, metaphase, anaphase and telophase. Postmitotic cells such as neurons are arrested in the quiescent stage G0.

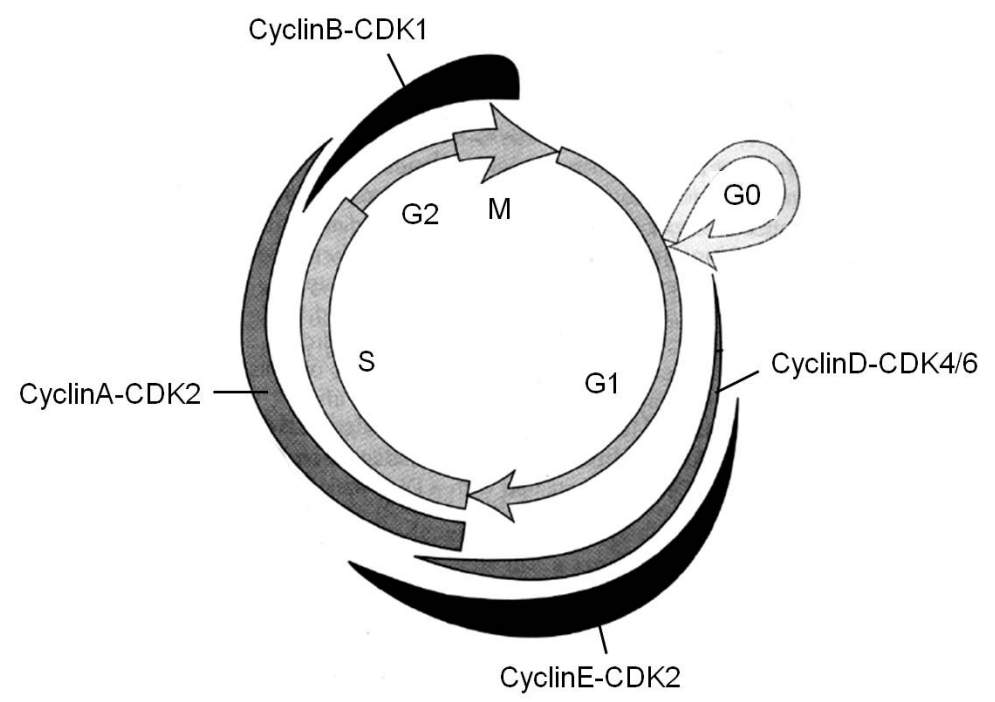

Figure 6: Cell cycle control by cyclin-CDK complexes.

Cyclin concentrations vary within the cell depending on the cell cycle phase. The association of cyclins with CDKs leads to activation of cell cycle related proteins and expression of cell cycle related genes (modified from Malumbres and Barbacid, 2009).

The transition of the cell cycle phases is regulated by the heterodimeric protein complexes consisting of cyclin-dependent kinases (CDKs) and the regulatory subunits cyclins (Figure 6). The cyclin concentration in the cells varies depending on the cell cycle phase and different cyclin-CDK complexes regulate the transition of specific cell cycle phases through activation of 
cell cycle related proteins and gene transcription. In G1-phase cyclinD is expressed and its encoded protein binds and activates CDK4 and CDK6 leading to activation of $\mathrm{Rb}$. The activation of $\mathrm{Rb}$ results in the expression of cyclinE. CyclinE binds and activates CDK2 an event essential for the G1/S-transition. During late stage of DNA replication, CDK2 forms a complex with cyclinA to enter the G2-phase. The cyclinB-CDK1 complex is the regulator of the mitotic entry and is needed for the progression through mitosis (Figure 6; reviewed in Malumbres and Barbacid, 2009).

\subsubsection{Regulation of mitotic entry}

In human cells the transcription of cyclinB starts in S-phase and peaks in late G2-phase. Its expression is controlled by several transcription factors including NF-Y, FoxM1 and B-Myb (Fung and Poon, 2005). There are two different cyclinB protein isoforms in mammalian cells, cyclinB1 and cyclinB2. CyclinB2 is non-essential and associates with the Golgi-apparatus to remodel the Golgi during mitosis. CyclinB1 is an essential protein that is important for CDK1 functions (Brandeis et al., 1998; Jackman et al., 1995). In S- and early G2-phases cyclinB1 shows a predominantely cytoplasmic localization (Yang et al., 1998), whereas in mid G2phase cyclinB1 starts to accumulate at the centrosomes. In late G2-phase the cyclinB1 concentration is highest at the centrosomes. The active cyclinB1-CDK1 complex appears first at the centrosomes to promote mitotic entry (Jackman et al., 2003). CDK1 is already bound to cyclinB in interphase (Bailly et al., 1989; Bailly et al., 1992), but CDK1 is phosphorlyated at T14 and Y15 resulting in inhibition of the kinase activity. The T14 and Y15 phoshorylation is controlled by the Wee1 and Myt1 kinases and the Cdc25 phosphatases. Wee1 and Myt1 phosphorylate CDK1 in G2-phase resulting in inhibition of cyclinB-CDK1 activity (O'Farrell, 2001). Once activated, cyclinB1-CDK1 can phosphorylate Wee1 and Myt1 leading to degradation of Wee1 and inhibition of Myt1 (Booher et al., 1997; Watanabe et al., 2005),

thereby amplifying CDK1 activity. The initial activation of centrosomal cyclinB1-CDK1 is controlled by the Cdc25B phosphatase through de-phosphorylation of T14 and Y15 in CDK1 (Jackman et al., 2003; Lammer et al., 1998; Lindqvist et al., 2005). There are three Cdc25 isoforms in human cells, Cdc25A, Cdc35B and Cdc25C that all show nuclear and cytoplasmic 
localization in G2-phase. Cdc25A is mainly nuclear, whereas Cdc25C is predominantly cytoplasmic. Both Cdc25B and Cdc25C are localized at the centrosomes in G2-phase (Busch et al., 2007; Dutertre et al., 2004). The activity of Cdc25B in turn is controlled by the Chk1 kinase that has additional nuclear functions in the DNA damage response (for review see Dai and Grant, 2010). Additionally to the nuclear localization, human Chk1 localizes to the centrosomes in interphase, but not mitotic cells (Kramer et al., 2004). Centrosome associated Chk1 inhibits the Cdc25B phosphatase through phosphorylation at S320 (Loffler et al., 2006; Schmitt et al., 2006). Activation of cyclinB1-CDK1 leads to phosphorylation of several substrates, which creates a Plk1-docking site on the CDK1-substrates (Elia et al., 2003a; Elia et al., 2003b). The activation of cyclinB1-CDK1 leads to its nuclear transport (Moore et al., 1999) and induction of mitotic events in the nucleus such as nuclear envelope breakdown that is triggered by cyclinB1-CDK1 substrates (Li et al., 1997). Overactive nuclear cyclinB1-CDK1 can promote premature chromosome condensation (Jin et al., 1998). However it is not clear whether the complex regulates the chromosome condensation in the normal cell cycle (Furuno et al., 1999; Hagting et al., 1999).

\subsubsection{Mitotic progression}

At the onset of mitosis, the chromatin condenses into chromosomes and the large protein complexes kinetochores start to assemble on centromeres in prophase (Morgan, 2007). At the transition of prophase to prometaphase, the nuclear envelope breaks down and the mitotic spindles, an array of microtubules, forms between two centrosomes that become the spindle poles. At metaphase, the kinetochores attach the centromeres to the plus-end of the spindle microtubules and the chromosomes become aligned (Cheeseman and Desai, 2008). The spindle assembly checkpoint monitors the correct alignment of the chromosomes and can block the transition to anaphase if sister chromatids are misaligned (Musacchio and Salmon, 2007). The progression into anaphase is controlled by the anaphase promoting complex/cyclosome bound to $\mathrm{Cdc} 20\left(\mathrm{APC} / \mathrm{C}^{\mathrm{Cdc} 20}\right)$ which targets several regulatory proteins for proteasomal degradation by ubiquitination. One target is securin and its degradation leads to chromosome segregation by dissolving the cohesin complex that holds the sister chromatids 
together (Nasmyth et al., 2000). Subsequently, kinetochores drive the sister chromatid segregation by motor activity (Cheeseman and Desai, 2008). At telophase, the mitotic spindles disassemble, the kinetochores dissociated, the nuclear envelope reforms and the chromosomes decondensate. Finally, the two daughter cells seperate during cytokinesis. These late mitotic events require the inactivation of the mitotic CDK1 complexes (Morgan, 2007).

\subsubsection{Role of centrosomes in the cell division}

\subsubsection{Centrosomal organization}

Centrosomes are small organelles within a cell and are usually localized in close proximity to the nucleus. Centrosomes consist of two main components in vertebrate cells, centrioles and the pericentriolar matrix. Centrioles are symmetrical barrel-shaped structures composed of nine sets of triplet microtubules. Each centrosome consists of two centrioles, the mother and daughter centriole which are organized perpendicular to each other and in close proximity at one end (proximal end). The mother centriole has additional appendages at the other end (distal end). The pericentriolar matrix is a network of fibers and protein aggregates surrounding the centrioles (Figure 7). The pericentriolar matrix serves as a scaffold for proteins which are involved in microtubule nucleation (for review on centrosomal structure see Doxsey, 2001). A ring-shaped muliprotein complex containing $\gamma$-tubulin ( $\gamma$-tubulin ring complex, $\gamma$-TuRC) is able to capture microtubule and serves as a template for the microtubule nucleation (Keating and Borisy, 2000; Moritz et al., 2000). The microtubules are attached at their plus-end to the kinetochores on chromosomes and at their minus-end to the centrosomes that form the mitotic spindle poles (Kitagawa and Hieter, 2001). The central role of centrosomes is the mitotic spindle assembly through their functions as spindle poles and microtubule nucleation and organization. However, centrosomes seem not essential for spindle assembly in mammalian cells, suggesting that centrosomes might ensure high fidelity of chromosome separation through spindle pole organization (Hinchcliffe et al., 2001; Khodjakov et al., 2000). 


\subsubsection{The centrosome cycle}

Centrosomes duplicate once every cell cycle. The timing of centrosome duplication is tightly controlled and is linked to the cell cycle (Figure 7). In G1-phase of the cell cycle, cells contain one centrosome. At the G1/S-transition, the two centrioles of one centrosome split and the mother and daughter centrioles can be distinguished. The mother centriole has appendages at the distal end and is associated with a subset of proteins such as ODF2/cenexin, ninein, centrin and $\varepsilon$-tubulin (Chang and Stearns, 2000; Lange and Gull, 1995; Mogensen et al., 2000; Paoletti et al., 1996). During S-phase of the cell cycle, both mother and daughter centrioles nucleate new centrioles, the procentrioles, at the side of the original centrioles. These procentrioles are then elongated during S-phase and G2-phase of the cell cycle (Chretien et al., 1997). The procentrioles reach full-length in late G2-phase in which also the final maturation takes place, with the recriutment of $\gamma$-TuRCs, centriolar and pericentriolar components (Andersen, 1999). At the G2/M-transition of the cell cycle, the duplicated centrosomes are separated by NEK2, a centrosomal kinase that separates the interconnecting material (Fry et al., 1998). After separation, the duplicated centrosomes move apart with the help of microtubule-dependent motor activities (Sharp et al., 2000) and recruit spindle pole components, such as NuMa and TPX2 to form the mitotic spindle poles (Figure 7; Du et al., 2001; Wittmann et al., 1998). 


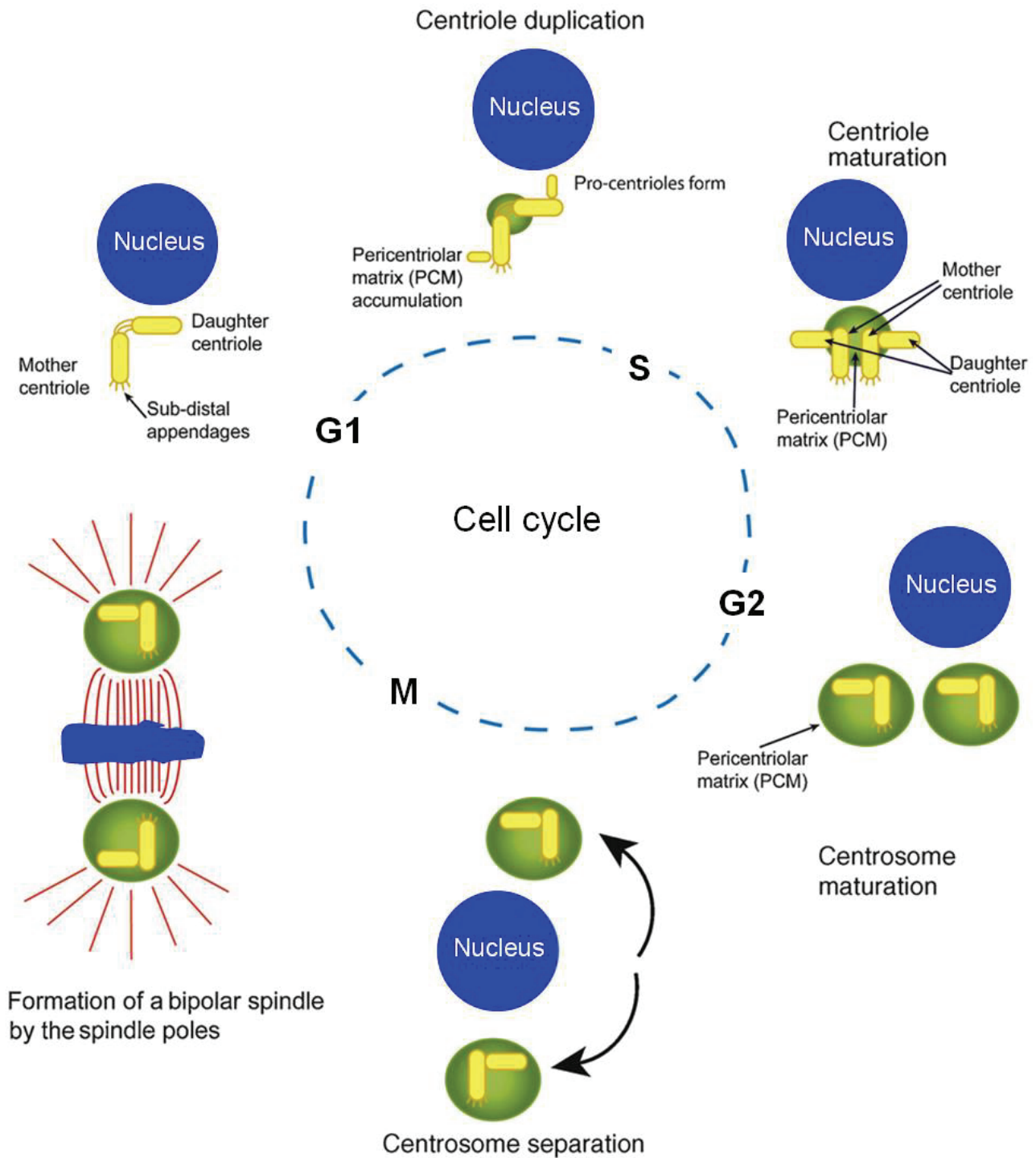

Figure 7: The centrosome cycle.

Schematic drawing of the centrosome cycle that is linked to the cell cycle (modified from Thornton and Woods, 2009). 


\subsection{Primary microcephaly}

\subsubsection{Clinical features of human primary microcephaly}

Microcephaly is a disease characterized by a reduced brain size and is defined by a reduced head circumference (occipito-frontal head circumference, OFC) of more than two standard deviations (SD) below the age and sex matched average (Figure 7). Patients with severe microcephaly show an OFC of less than three standard deviations. Hereditary microcephaly is distinguished between primary microcephaly that is apparent at birth and secondary microcephaly which become apparent postnatally. Primary microcephaly is a disorder of defective prenatal neurogenesis, whereas secondary microcephaly indicates a progressive neurodegenerative disease (Woods, 2004). In addition to a reduced OFC, autosomal recessive primary microcephaly $(\mathrm{MCPH})$ is characterized by mild to moderate mental retardation (IQ between $30 \%$ and $80 \%$ of average) but otherwise normal in health and neurological functions (Cox et al., 2006; Woods et al., 2005). In certain cases of MCPH speech delay, hyperactivity, attention deficit and aggressiveness are reported and in some patients, abnormal body height and weight was detected (Passemard et al., 2009). The incidence of $\mathrm{MCPH}$ is $1: 30,000$ to $1: 250,000$ per live-birth depending on the population (Woods et al., 2005). Imaging studies showed that MCPH patients have a reduced brain size with normal architecture. However, the cerebral cortex shows the greatest size reduction (Figure 8; Kaindl et al., 2010; Woods et al., 2005). Moreover, enlarged lateral ventricles and agenesis of the corpus callosum were detected (Passemard et al., 2009).

Mutations in seven gene loci cause the MCPH subtypes 1 to 7 . The genes encode the proteins MCPH1/microcephalin/BRIT1 (MCPH1; Jackson et al., 2002; Jackson et al., 1998), WD-40 repeat protein WDR62 (MCPH2; Nicholas et al., 2010; Yu et al., 2010), cyclin-dependent kinase 5 regulatory associated protein 2 CDK5RAP2 (MCPH3; Bond et al., 2005; Moynihan et al., 2000), centrosomal protein CEP152 (MCPH4; Guernsey et al., 2010; Kalay et al., 2011), abnormal spindle-like microcephaly associated ASPM (MCPH5; Pattison et al., 2000; Shen et al., 2005), centromeric protein J CENPJ (MCPH6; Bond et al., 2005; Leal et al., 2003) and SCL/TAL1-interrupting locus STIL (MCPH7; Kumar et al., 2009). It has been suggested that the reduced brain size of $\mathrm{MCPH}$ patients is the result of abnormal neuronal progenitor cell 
proliferation. Since all MCPH proteins seem to associate with either the centrosomes or the mitotic spindles suggests a specific role of these proteins during mitosis in the period of neurogenesis (Kaindl et al., 2010; Thornton and Woods, 2009; Woods et al., 2005). The $\mathrm{MCPH}$ proteins may played a role in the evolution of the cerebral cortex (Ponting and Jackson, 2005; Woods et al., 2005). The massive increase of the cerebral cortex size during evolution correlates with an increase of behavioral and cognitive capacity. Obvious candidates for the evolutionary changes of the cerebral cortex size are the MCPH genes.

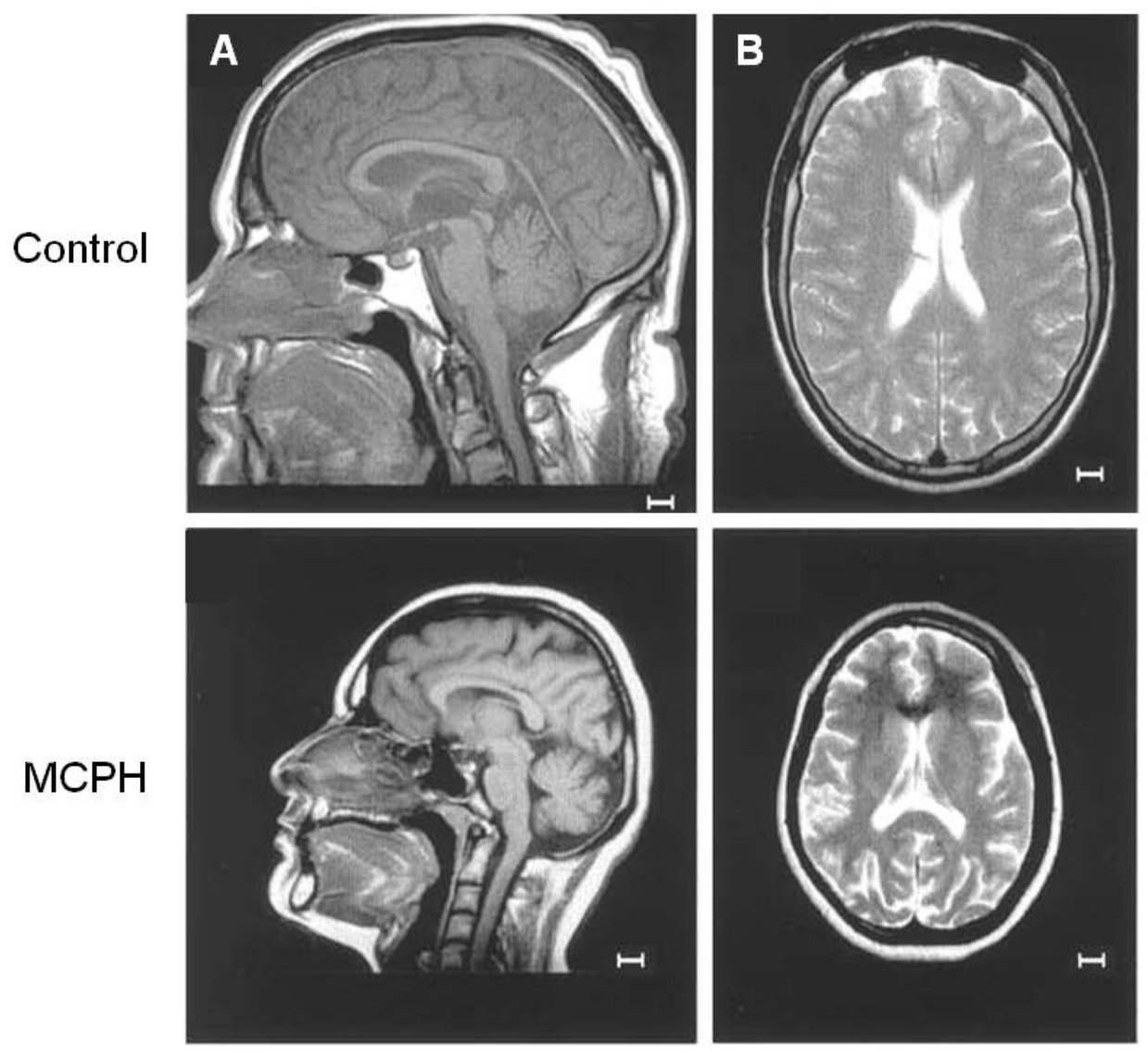

Figure 8: Reduced cerebral cortex size of MCPH patient.

NMRI (nuclear magnetic resonance imaging) scan of an adult MCPH patient with mutations in the Microcephalin gene (see chapter 1.3.2) compared to an age matched control (modified from Trimborn et al., 2004).

(A) Sagittal T1-weighted images. Scale bars: $1 \mathrm{~cm}$.

(B) Axial T2-weighted images. Scale bars: $1 \mathrm{~cm}$. 


\subsubsection{MCPH1/microcephalin/BRIT1}

The first mutations identified that lead to MCPH were within the 14 exon gene Microcephalin, localized on chromosome 8p23. Microcephalin encodes the protein MCPH1 also referred to as microcephalin (Jackson et al., 2002; Jackson et al., 1998). Microcephalin is additionally referred to as BRIT1 (BRCT-repeat inhibitor of $h$ Tert expression), since it was independently identified by a genetic screening for negative regulators of $h$ Tert expression which encodes the catalytic subunit of human telomerase (Lin and Elledge, 2003). Up to date, four Microcephalin gene mutations were described that lead to $\mathrm{MCPH}$, that are $74 \mathrm{C}>\mathrm{G}$ with an OFC between -5 to -10 SD (Jackson et al., 2002; Jackson et al., 1998), 80C>G with an OFC of -3 SD (Trimborn et al., 2005), ins427A with an OFC between -10 to -12 SD (Neitzel et al., 2002; Trimborn et al., 2004) and a mutation linked to the Microcephalin locus with an OFC between -7 to -10 SD (Roberts et al., 2002).

Microcephalin mRNA is expressed in human fetal brain, fetal liver and fetal kidney but is also detected in numerous adult tissues (Jackson et al., 2002). The mouse homolog of Microcephalin, Mcph1 was shown to be highly expressed in the forebrain in proximity to the lateral ventricles during the period of embryonic neurogenesis (Jackson et al., 2002).

The human MCPH1 protein consists of 835 amino acids and has three BRCT (C-terminal domain of breast cancer gene 1, BRCA1) domains, one in the N-terminus and two in the Cterminus with presumably different functions. BRCT1 domains are phosphopeptide-binding domains that a present in a variety of proteins with roles in the cell cycle checkpoints and DNA damage repair (Manke et al., 2003; Yu et al., 2003). The cell cycle checkpoint mechanisms are linked to DNA damage repair mechanisms in order to delay the cell cycle progression until the damage is repaired (see Warmerdam and Kanaar, 2010 for review on cell cycle checkpoints and DNA repair).

Previous studies showed that the two C-terminal BRCT domains of MCPH1 are involved in the DNA damage response. MCPH1 is recruited to DNA damage sites to form protein foci through binding of these BRCT domains to $\gamma-\mathrm{H} 2 \mathrm{AX}$ (Jeffers et al., 2008; Wood et al., 2007), a histone variant phosphorylated at the DNA damage site (Rogakou et al., 1998). The MCPH1 foci formation seems to be upstream of both main DNA damage response pathways: ATM- and ATR-related DNA damage responses. Because MCPH1 is required for the recruitment of DNA damage signaling factors involved in both pathways, such as RAD51, 53BP1, MDC1, 
phosphorylated NBS1, RPA and RAD17 (Rai et al., 2006; Wood et al., 2008; Wood et al., 2007; Wu et al., 2009). However, in a study by Alderton et al., it was shown that MCPH1 functions specific in the ATR branch of the DNA damage response in regulating cell cycle arrest through direct binding to Chk1 (Alderton et al., 2006). Lymphoblastoid cells from MCPH patients with mutation in Microcephalin were shown to be defective in the cell cycle checkpoint arrest at the G2/M-boundary after DNA damage. The MCPH1 patient cells showed impaired degradation of Cdc25A and fail to inhibit the loading of Cdc45 to chromatin after drug induced replication arrest (Alderton et al., 2006).

Additionally, MCPH1 was shown to regulate transcription of DNA damage response genes as well as apoptotic genes through interaction of its two C-terminal BRCT domains with the E2F1 transcription factor. $\mathrm{MCPH} 1$ together with $\mathrm{E} 2 \mathrm{~F} 1$ are able to bind to the promoters of $B R C A 1$, CHK1, DDB2, RAD51 and caspase7 to regulate their gene expression (Yang et al., 2008).

The N-terminal BRCT domain of MCPH1 was shown to interact with the switch/sucrose nonfermentable (SWI/SNF) chromatin remodeling complex to regulate chromatin relaxation which facilitates access of DNA damage repair proteins (Peng et al., 2009).

Moreover, the N-terminal BRCT domain of MCPH1 seems to play a role in centrosomal and cell cycle regulatory functions. MCPH1 localizes to the centrosomes throughout the cell cycle in human U2OS cells (Zhong et al., 2006). Consistently, the homologues of MCPH1 in Drosophila melanogaster and in chicken DT40 cells were also shown to have centrosomal localization (Brunk et al., 2007; Jeffers et al., 2008; Rickmyre et al., 2007). It was shown, using different MCPH1 constructs and chicken DT40 cells, that specific the N-terminal BRCT-domain is important for its centrosomal localization (Jeffers et al., 2008). Knockdown experiments of Microcephalin in human U2OS cells induced centrosomal anomalies, mitotic spindle misalignment and delayed cytokinesis (Rai et al., 2008). Therefore, two potential roles of $\mathrm{MCPH} 1$ have emerged, one in the DNA damage response and one in the control of the cell cycle without DNA damage.

One hallmark of $\mathrm{MCPH}$ patient cells with Microcephalin gene mutations is premature chromosome condensation, prior to mitosis and a delayed chromosome decondensation after completion of mitosis (Neitzel et al., 2002; Trimborn et al., 2004). The premature condensation of chromosomes in early G2-phase coincides with a reduced inhibitory Y15 phosphorylation of CDK1 that prevents mitotic entry (Alderton et al., 2006). It was shown that MCPH1 via a middle domain can bind to Condensin II, a protein important for chromosome condensation, 
which might explain the premature chromosome condensation phenotype (Trimborn et al., 2006; Wood et al., 2008).

The Drosophila melanogaster homologue, Mcph1 seems to be important for the coordination of mitosis in the embryos. Deletion of mcph1 in Drosophila melanogaster results in slower mitotic progression and detachment of the centrosomes (Brunk et al., 2007; Rickmyre et al., 2007). However, the brains of mcph1 mutant flies showed a normal size (Rickmyre et al., 2007). Recently, two studies of mouse models with knockout of the Mcph1 gene have been published. Although, cells isolated from the Mcph1 knockout mice resemble the chromosome condensation defects of the human MCPH1 patients cells, these mice showed a normal brain development (Liang et al., 2010; Trimborn et al., 2010). Hence, the mechanisms underlying the neurodevelopmental defects leading to a reduced brain size in human MCPH1 patients are still elusive.

\subsubsection{MCPH2/WDR62}

The gene responsible for $\mathrm{MCPH} 2$ was mapped to chromosome $19 q 12$ and was recently identified as WDR62. The WDR62 gene consists of 32 exons and encodes a protein of 1,518 amino acids containing 15 WD40 repeats (Nicholas et al., 2010; Yu et al., 2010). Previously, WDR62 was identified as a binding partner of c-Jun N-terminal kinase (JNK), suggesting a role in potentiating JNK activity (Wasserman et al., 2010). In another study, WDR62 was identified as a protein binding to centrosomal protein CEP170 (Hutchins et al., 2010).

In situ hybridization with a probe to mouse Wdr62 showed a widespread expression in the embryonic brain with the highest expression in the forebrain (Yu et al., 2010). Analysis of mouse embryonic brain sections with WDR62-antibody showed an upregulation of WDR62 protein level in mitotic neuronal progenitor cells (Nicholas et al., 2010). The subcellular localization of WDR62 shows cell cycle-dependency. In human HeLa cells, WDR62 is localized diffusely in the cytoplasm and to the Golgi-apparatus in interphase, whereas in Mphase WDR62 is found at the spindle poles (Nicholas et al., 2010; Yu et al., 2010). Up to date there is not much known about the molecular function of WDR62. However, it seems that 
WDR62 has a role at the spindle poles of mitotic neuronal progenitor cells which might affect the proliferation and neuron production during embryonic neurogenesis.

\subsubsection{MCPH3/CDK5RAP2}

Mutations in the human gene encoding the protein cyclin-dependent kinase 5 , regulatory associated protein 2 (CDK5RAP2), also referred to as centrosomal protein 215 (CEP215), causes MCPH3 (Bond et al., 2005; Moynihan et al., 2000). The 34-exon gene located on human chromosome 9q34 is widely expressed in human tissues (Bond et al., 2005; Ching et al., 2000). The mouse homologue is expressed in many embryonic tissues with the highest expression level in the forebrain in close proximity to the lateral ventricles (Bond et al., 2005; Buchman et al., 2010), but undetectable in the adult mouse brain (Lizarraga et al., 2010).

The human CDK5RAP2 protein consists of 1,893 amino acids and its C-terminus interacts with CDK5 activator protein, also referred to as CDK5 regulatory subunit 1 (CDK5R1), thereby indirectly inhibiting CDK5 via inhibition of CDKR1 (Ching et al., 2000; Wang et al., 2000). CDK5 is an unusual member of the CDK protein family, since it seems dispensable for the cell cycle regulation, unlike other CDKs (Dhariwala and Rajadhyaksha, 2008). It was shown that nuclear localization of CDK5 allows neurons to remain postmitotic in vitro (Zhang et al., 2008). In addition, CDK5 has a role in neuronal migration (Ohshima et al., 1996).

At the N-terminus, human CDK5RAP2 protein contains a $\gamma$-TuRC binding site. CDK5RAP2 is associated with the centrosomes throughout the cell cycle in HeLa cells and is required to connect $\gamma$-TuRC to the centrosomes (Fong et al., 2008). This connection is likely achieved through direct interaction of CDK5RAP2 with pericentrin, since deletion of pericentrin leads to loss of CDK5RAP2 from the centrosome in U2OS cells (Graser et al., 2007). Deletion of the CDK5RAP2 homologue cnn, encoding the protein centrosomin in Drosophila melanogaster leads to the loss of the connection between centrioles and the pericentriolar matrix in the mutant cells. However, the centrosomin mutant flies showed a normal brain development (Lucas and Raff, 2007). In mouse fibroblast it was shown that CDK5RAP2 is localized to the centrosomes throughout the cell cycle and its centrosomal level is regulated in a cell cycledependent manner. CDK5RAP2 protein level is low in interphase, increases in prophase and 
remains high during mitosis (Barrera et al., 2010). In the study of Barrera et al., the generation of Cdk5rap2 knockout mice was described. Cdk5rap2-deficiency results in centriole disengagement leading to centriole amplification and multipolar spindle poles of mitotic mouse embryonic fibroblasts (MEFs). However, these mice do not show any evidence of reduced brain size (Barrera et al., 2010). Recently, it was shown by another study that the Hertwig's anemia mouse that arose in the progeny of an irradiated mouse (Hertwig, 1942), is due to mutations in Cdk5rap2 (Lizarraga et al., 2010). Additionally to the previously described anemia phenotype, the Cdk5rap2 mutant mice show severe neurological defects due to abnormal cortical development leading to a reduced brain size present at birth (Lizarraga et al., 2010). During neurogenesis, the Cdk5rap2-deficient cortical neuronal progenitor cells show defective mitotic spindles resulting in increased cell death and a premature cell cycle exit and thereby an insufficient production of cortical neurons (Lizarraga et al., 2010). Similarily, using in utero electroporation technique to knockdown Cdk5rap2 in the developing mouse embryonic forebrain, Buchman et al., showed an increased differentiation phenotype of cortical neuronal progenitor cells during neurogenesis (Buchman et al., 2010). The CDK5RAP2 interaction with pericentrin seems to be important for the role of CDK5RAP2 during neurogenesis, since knockdown of pericentrin by the same technique phenocopies the Cdk5rap2 knockdown on cortical neuronal progenitor cell differentiation (Buchman et al., 2010).

\subsubsection{MCPH4/CEP152}

The gene responsible for $\mathrm{MCPH} 4$ was recently identified as CEP152, encoding the centrosomal protein CEP152 (Kalay et al., 2011). However, another study identified CEP152 mutated in Seckel syndrome patients (Guernsey et al., 2010). Seckel syndrome is a disorder of impaired DNA damage response associated with genomic instability (see Kerzendorfer and O'Driscoll, 2009 for review).

The mouse gene is expressed in embryonic brain tissue (Guernsey et al., 2010). The human 27-exon gene encodes a 1,654 amino acid protein which was previously identified as a centrosomal protein (Andersen et al., 2003). The centrosomal localization of human CEP152 was confirmed by overexpression analysis and antibody-staining (Guernsey et al., 2010; Kalay 
et al., 2011). CEP152-deficient human fibroblasts showed fragmented centrosomes in interphase and mono- as well as multipolar spindle and misaligned spindles in metaphase (Kalay et al., 2011). The Drosophila melonogaster homologue of CEP152, asterless was shown to localize to the centrosomes at the periphery of the centrioles to function in the initiation of centriole duplication (Blachon et al., 2008). However, it is not known which function of CEP152 is important for normal brain development.

\subsubsection{MCPH5/ASPM}

The most common cause of MCPH is mutation of the human ASPM gene that is located on chromosome 1q31 and encodes abnormal spindle-like, microcephaly-associated protein ASPM (Pattison et al., 2000; Shen et al., 2005). The human 28-exon ASPM gene is expressed in proliferating tissues and the mouse homologue Aspm was shown to be highly expressed in the VZ of the embryonic dorsal telencephalon (Fish et al., 2006; Kouprina et al., 2005).

The human ASPM protein consists of 3,477 amino acids containing a putative $\mathrm{N}$-terminal microtubule binding domain, two calponin homology $(\mathrm{CH})$ domains and up to 81 calmodulinbinding IQ motifs (Bond et al., 2002; Kumar et al., 2002; Saunders et al., 1997). ASPM localizes to the centrosomes throughout the cell cycle in human U2OS cells as well as in mouse neuronal progenitor cells (Fish et al., 2006; Zhong et al., 2005). ASPM functions in the maintenance of symmetric cell divisions of NEs and RGCs and is downregulated with the switch from proliferative to neurogenic cell divisions (Fish et al., 2006). Knock-down of mouse Aspm in the embryonic dorsal telencephalon during neurogenesis by in utero electroporation technique resulted in an alteration of the mitotic cleavage plane of neuronal progenitor cells thereby increasing the asymmetric cell division mode (Fish et al., 2006). ASPM might not be only responsible for mitotic spindle and cleavage plane positioning, but also for the centrosome function as a microtubule organization center, as suggested from a study of Drosophila melanogaster asp ${ }^{-/}$mutants (Casal et al., 1990). Further insights into the mechanism of ASPM in spindle organization and positioning comes from a study in Caenorhabditis elegans from van der Voet et al. In this study, the ASPM homologue promotes the meiotic spindle organization through accumulation of LIN-5, the homologue of human 
spindle apparatus protein NuMa, to the spindle poles together with calmodulin (van der Voet et al., 2009). This study suggests the existence of two different complexes important for dynein function to regulate spindle rotation, the LIN-5/GPR-1/-2/G $\alpha$ complex at the cell cortex (see chapter 1.1.5.3) and the LIN-5/ASPM/calmodulin at the spindle poles (van der Voet et al., 2009). However, it is still unknown whether the functional interactions of ASPM are conserved in mammals and how this mechanism might affect the cell division mode of neuronal progenitor cells and neurogenesis. It was shown recently, that mutations in mouse Aspm gene result in a mild reduction of brain size, although not comparable with the severity of human primary microcephaly (Pulvers et al., 2010). The analysis of the cleavage plane of mitotic neuronal progenitor cells revealed no significant alteration which is in contrast to the in vivo Aspm knockdown experiments (Fish et al., 2006; Pulvers et al., 2010). The differences might be explained by the production of truncated ASPM protein isoforms in the mutant mice which were shown to consist of the N-terminal microtubule binding domain. The truncated ASPM isoforms were still localized to the centrosomes and might be partial functional to regulate the mitotic spindle orientation (Pulvers et al., 2010).

\subsubsection{MCPH6/CENPJ}

The human gene CENPJ is responsible for MCPH6 when mutated (Bond et al., 2005; Gul et al., 2006). The 17-exon gene encodes the centromeric protein J (CENPJ), also referred to as centrosomal P4.1-associated protein (CPAP) and LAD-3-associated protein (LAP), consisting of 1,338 amino acids. CENPJ contains a microtubule destabilizing motif (PN2-3) that inhibits microtubule assembly and interacts with 14-3-3 in a cell cycle-dependent manner (Chen et al., 2006; Hung et al., 2004). The subcellular localization of CENPJ was shown to be centrosomal throughout the cell cycle in a human cervical carcinoma cell line where it interacts with the $\gamma$ TuRC (Hung et al., 2000). The role of CENPJ at the centrosomes has been suggested to be the regulation of microtubule assembly and disassembly through its PN2-3 motif which is important for spindle maintenance (Hung et al., 2004). Downregulation of CENPJ leads to multipolar spindle poles, mitotic arrest and apoptosis (Cho et al., 2006). Moreover, CENPJ has been suggested to be a co-activator of NF-kB, a transcription factor important for inflammation, 
immune response, proliferation and apoptosis. This function seems to be achieved through interaction of CENPJ with the co-activator p300/CREB-binding protein (Koyanagi et al., 2005). Deletion of the Drosophila melanogaster CENPJ homologue Dsas-4 (spindle assembly abnormal), results in loss of centrioles during embryonic development. In these mutant cells the mitotic spindle assembly is slow and asymmetric cell divisions of neuroblasts are abnormal, but without affecting the brain size (Basto et al., 2006; Stevens et al., 2007). The CENPJ homologue in Caenorhabditis elegans, sas-4 is a centriolar protein which controls the size, organization and duplication of the centrosomes (Kirkham et al., 2003; Leidel and Gonczy, 2003). It was further shown that sas-4 is recruited to the centrosomes at the onset of centrosomal duplication in S-phase of the cell cycle and it is essential for microtubule assembly (Dammermann et al., 2008; Leidel and Gonczy, 2003).

\subsubsection{MCPH7/STIL}

MCPH7 is caused by mutations in the human 18-exon gene STIL (SCL/TAL1 interrupting locus), also referred to as SIL (Kumar et al., 2009). STIL was previously identified at the site of genomic rearrangement in T-cell acute lymphoblastic leukemia (Aplan et al., 1990; Aplan et al., 1992; Brown et al., 1990).

Human STIL is expressed in proliferating cells during early embryonic development (Izraeli et al., 1997; Izraeli et al., 1999). Like other MCPH genes, the mouse homologue Stil has been shown to be expressed in the embryonic cerebral cortex during neurogenesis (Smith et al., 2007b). However, Stil is expressed in many mouse tissues with the highest levels in the bone marrow, thymus and spleen (Collazo-Garcia et al., 1995). STIL expression is regulated by the E2F transcription factor and is required for the E2F induced mitotic progression (Erez et al., 2007). The human STIL protein consists of 1,287 amino acids containing a putative nuclear localization signal and a domain at the C-terminus that is similar to TGF- $\beta$ (Karkera et al., 2002). In HeLa cells, STIL was detected at the mitotic spindle poles in metaphase, whereas anaphase cells lacked STIL protein at the spindle poles and interphase cells showed almost no detectable protein level (Pfaff et al., 2007). STIL does not completely colocalize with $\gamma$ tubulin at the spindle poles but rather with the pericentriolar matrix (Pfaff et al., 2007). STIL is 
phoshorylated in mitosis to promote the interaction with Pin1 which seems to be required for the spindle assembly checkpoint (Campaner et al., 2005).

Knockdown of STIL in HeLa cells resulted in disorganized mitotic spindles. Similarily, zebrafish Danio rerio mutants for cassiopeia (csp), the homologue of STIL, showed a high number of mitotic cells with disorganized spindles and moreover often lost either one or both centrosomes (Pfaff et al., 2007). The STIL knockout mice show developmental abnormalities around E7.5 and die at E10.5. The number of cells in the neuroepithelium is reduced in the Stil mutants, which is caused by a decreased proliferation and an increase in apoptosis and Stir ${ }^{--}$ embryos fail to close the neural tube. In addition, Stir ${ }^{--}$mice display abnormal left-right specification as a result of blocked Sonic Hedgehog (Shh) signaling (Izraeli et al., 2001; Izraeli et al., 1999). The discrepancy between the human and mouse phenotypes is currently not understood.

\subsubsection{Common role of $\mathrm{MCPH}$ proteins in cortical development}

The MCPH proteins are not members of a common protein family and have multiple seemingly unrelated molecular functions, such as control of cell cycle progression, DNA damage repair, DNA damage checkpoints, spindle assembly checkpoint, regulation of gene transcription, centrosome maturation and organization, mitotic spindle integrity and control of progenitor proliferation. It seems surprising that all patients suffering from the seven MCPH subtypes are characterized by the same phenotype of reduced cerebral cortex size. However, it is not known whether MCPH1 proteins share a common mechanism to ensure a normal brain development. Interestingly, all MCPH proteins associate with the centrosomes throughout the cell cycle or specific during mitosis. This suggests an important function of the centrosomes and centrosomal proteins of neuronal progenitor cells during the generation of neurons. It has been suggested that the reduced brain size of $\mathrm{MCPH}$ patients is the result of abnormal neuronal progenitor cell proliferation in which the centrosomes play important roles (Kaindl et al., 2010; Thornton and Woods, 2009; Woods et al., 2005). 


\subsection{Aim of the study}

Mutations in the human Microcephalin gene cause the neurodevelopmental disorder MCPH1 characterized by a reduced brain size apparent at birth. Previous studies showed that the protein MCPH1, encoded by Microcephalin displays multiple functions in the response to DNA damage as well as in the regulation of the cell cycle as component of the centrosomes. However, the mechanisms underlying the neurodevelopmental defects leading to a reduced brain size in human MCPH patients are not known. It has been speculated that defective DNA damage response during neurogenesis may lead to cell cycle arrest and increased cell death resulting in a reduced number of neurons produced (McKinnon 2009). Another hypothesis is an alteration of the cell division mode of the neuronal progenitor cells which would result in an insufficient production of neurons (Thornton and Woods, 2009; Woods 2005). The aim of this study is to identify the basis of the developmental defects leading to a reduced brain size due to Mcph1 deficiency. Identifying these mechanism might also help to understand the role of other MCPH genes in the development of the CNS. The specific questions to be addressed by this study are:

i) Does deletion of the Mcph1 gene in mice lead to a reduced brain size resembling the human phenotype?

ii) What are the developmental defects occurring during embryonic neurogenesis caused by Mcph1 deficiency?

iii) What of the multiple described molecular functions of $\mathrm{MCPH} 1$ are responsible for the brain size determination in mouse? 


\section{Material and Methods}

\subsection{Material}

\subsubsection{Cell culture media and solutions}

Mouse embryonic fibroblast (MEF) medium: Dulbecco's Modified Eagle Medium (DMEM; Invitrogen), containing $10 \%$ fetal calf serum (FCS; LONZA), 1 mM sodium pyruvate (Invitrogen), $2 \mathrm{mM} \mathrm{L-glutamine} \mathrm{(Invitrogen),} 100$ units/mL Penicillin (Invitrogen), $100 \mu \mathrm{g} / \mathrm{mL}$ Streptomycin (Invitrogen) and $0.5 \mathrm{mM} \beta$-mercaptoethanol (Invitrogen).

MEF freezing medium: DMEM (Invitrogen) containing $10 \%$ FCS (LONZA) and $10 \%$ dimethylsulfoxid (DMSO; Sigma-Aldrich).

Mouse embryonic neuronal progenitor medium: DMEM-NutMix/F12-GlutaMAX medium (Invitrogen) containing B-27 serum-free supplement (Invitrogen), $10 \mathrm{ng} / \mathrm{mL}$ epidermal growth factor (EGF; Peprotech), $20 \mathrm{ng} / \mathrm{mL}$ fibroblast growth factor-basic (bFGF; Peprotech), 100 units $/ \mathrm{mL}$ Penicillin (Invitrogen) and $100 \mu \mathrm{g} / \mathrm{mL}$ Streptomycin (Invitrogen).

Neurobasal medium: Neurobasal medium (Invitrogen) containing B-27 serum-free supplement (Invitrogen), 2 mM L-glutamine (Invitrogen) and 10 ng/mL bFGF (Peprotech)

Mouse embryonic brain digestion solution: DMEM-NutMix/F12-GlutaMAX medium (Invitrogen) containing 30 units $/ \mathrm{mL}$ papain (Sigma-Aldrich), $240 \mu \mathrm{g} / \mathrm{mL}$ cystein (Sigma-Aldrich) and $400 \mu \mathrm{g} / \mathrm{mL}$ DNAse I type IV (Roche Diagnostics).

Ovomuccoid trypsin inhibitor solution: Leibovitz's L15 medium (Invitrogen) containing $1.125 \mathrm{mg} / \mathrm{mL}$ Ovomuccoid trypsin inhibitor (Worthington Biochemical), $525 \mu \mathrm{g} / \mathrm{mL}$ bovine serum albumin (BSA; Sigma-Aldrich) and $40 \mathrm{mg} / \mathrm{mL}$ DNAse I type IV (Roche Diagnostics). 


\subsubsection{Antibodies}

In Table 1 all primary antibodies are shown and in Table 2 all secondary antibodies are listed.

\begin{tabular}{|c|c|c|c|}
\hline Antigen & Species and antibody & Company & Purpose and dilution \\
\hline$\alpha$-tubulin (clone B5-1-2) & Mouse monoclonal & Sigma-Aldrich & IHC 1:300; IC 1:500 \\
\hline$\beta$-actin (clone AC-15) & Mouse monoclonal & Sigma-Aldrich & WB 1:30,000 \\
\hline BrdU (clone BU1/75 [ICR 1]) & Rat monoclonal & Abcam & IHC 1:100 \\
\hline$\beta$-tubulinIII & Rabbit polyclonal & Sigma-Aldrich & IHC 1:200 \\
\hline Cdc25B & Rabbit polyclonal & Cell Signaling & WB $1: 1,000$ \\
\hline Cdk1-Y18p & Rabbit polyclonal & Cell Signaling & WB $1: 1,000$ \\
\hline Chk1 & Rabbit polyclonal & Cell Signaling & IC 1:100 \\
\hline Chk1 & Sheep polyclonal & Abcam & WB $1: 1,000$ \\
\hline$\gamma-\mathrm{H} 2 \mathrm{AX}$ & Rabbit polyclonal & Millipore & IHC 1:500 \\
\hline$\gamma$-tubulin (clone GTU-88) & Mouse monoclonal & Sigma-Aldrich & IC $1: 300$ \\
\hline Ki67 & Rabbitpolyclonal & Fremont NeoMarkers & $\operatorname{IHC} 1: 100$ \\
\hline $\mathrm{N}$-cadherin (clone 32) & Mouse monoclonal & Becton Dickinson & IHC 1:200 \\
\hline Nestin (clone Rat-401) & Mouse monoclonal & Millipore & IC 1:300 \\
\hline ODF2/cenexin & Rabbit polyclonal & Abcam & IC 1:200 \\
\hline Pan-cadherin & Rabbitpolyclonal & Abcam & IHC 1:300 \\
\hline Pericentrin & Rabbit polyclonal & Covance & IC 1:200 \\
\hline pH3 (phospho-S10 Histone3) & Rabbit polyclonal & Millipore & IC $1: 200$ \\
\hline
\end{tabular}




\begin{tabular}{|c|c|c|c|}
\hline Antigen & Species & Conjugate & Company \\
\hline Mouse $\lg G \mathrm{~F}\left(\mathrm{ab} \mathrm{b}^{\prime}\right)_{2}$ & Sheep & Сy3 & Sigma-Aldrich \\
\hline Mouse IgG $F\left(a b^{\prime}\right)_{2}$ & Sheep & FITC & Sigma-Aldrich \\
\hline Rabbit IgG $F\left(a b^{\prime}\right)_{2}$ & Sheep & Суз & Sigma-Aldrich \\
\hline Rabbit IgG $F\left(a b^{\prime}\right)_{2}$ & Goat & FITC & Sigma-Aldrich \\
\hline Rat IgG $F\left(a b^{\prime}\right)_{2}$ & Goat & Cy2 & $\begin{array}{l}\text { Jackson } \\
\text { ImmunoResearch }\end{array}$ \\
\hline Sheep whole IgG & Rabbit & HRP & Dako \\
\hline Rabbit whole IgG & Goat & HRP & Dako \\
\hline Mouse whole IgG & Goat & HRP & Dako \\
\hline
\end{tabular}

\subsubsection{Animals}

The Mcph1 conventional knockout line $M c p h 1^{\Delta / \Delta}$, as well the conditional knockout lines $M c p h 1^{\text {floxfliox }}$ and $M c p h 1^{T / T}$ were generated by gene targeting in mouse embryonic stem (ES) cells. The ES cells of 129/Sv origin (clone E14.1) carrying Mcph1 targeted allele $\left(\mathrm{Mcph}^{+/ T}\right)$ were generated by electroporation of a targeting vector that has been engineered using the recombineering technique (Sukchev, 2010). The targeted ES cells have been injected into blastocysts of C57/BL6 background to obtain chimeric mice that were backcrossed with C57/BL6 mice. Offsprings were intercrossed for colony maintenance. The targeted (T) and floxed (flox) alleles carry two loxP-sites flanking exons 4 and 5 of Mcph1 gene. The targeted allele contains in addition a neo-resistance gene. The deleted allele $(\Delta)$ was generated by Cremediated recombination to excise exons 4 and 5 leading to the generation of several premature stop codons in exon 6 of the Mcph1 gene (Sukchev, 2010). The transgenic mouse line nestin-Cre express the Cre gene, encoding a recombinase under the control of the nestin 
promoter that is specific to neuronal progenitor cells (Tronche et al., 1999). Expression of nestin-Cre is high in the neural tube as early as E9.5 (Zimmerman et al., 1994).

All animals were maintained under specific pathogen-free conditions. Animal care and experiments were performed in accordance with the ethic committee guidelines.

\subsubsection{Microscopy}

Fluorescence images were taken with either a fluorescence microscope (Zeiss Axiolmager M1), a fluorescence microsocope capable of generating optical slices (Zeiss Apotome) or a laser scanning confocal microsocope (LSM 510, Zeiss). Pictures were taken with a monochrome CCD (charge-coupled device)-camera (Zeiss AxioCam MRm). For brightfield images, a brightfield microscope (Zeiss Axiolmager M1) together with a CCD-camera (Zeiss AcioCam MRc5) was used.

\subsection{Molecular biological methods}

\subsubsection{DNA extraction}

DNA extraction was used for the genetic analysis of the mouse colonies by polymerase chain reaction. Mouse tail biopsies were digested overnight at $55^{\circ} \mathrm{C}$ in lysis buffer $\left(\mathrm{H}_{2} \mathrm{O}\right.$ containing $50 \mathrm{mmol} / \mathrm{L}$ Tris- $\mathrm{HCl}, 100 \mathrm{mmol} / \mathrm{L}$ EDTA, $100 \mathrm{mmol} / \mathrm{L} \mathrm{NaCl}, 1 \%$ sodium dodecyl sulfate (SDS) and $500 \mu \mathrm{g} / \mathrm{L}$ Proteinase $\mathrm{K}$ ). For protein denaturation $200 \mu \mathrm{L}$ of saturated $\mathrm{NaCl}$ solution was added and incubated for $5 \mathrm{~min}$ at room temperature (RT) with shaking. The mixture was centrifuged at $18,400 \times \mathrm{g}$ for $10 \mathrm{~min}$ at room temperature to remove the precipitate. To precipitate the DNA, $700 \mu \mathrm{L}$ of the supernatant was transferred to a fresh $1.5 \mathrm{~mL}$ Eppendorftube and $400 \mu \mathrm{L}$ of iso-propanol was added. The mixture was incubated for 2 min at RT with 
shaking followed by centrifugation at $18,400 \times \mathrm{g}$ for $10 \mathrm{~min}$ at RT. The supernatant was removed, the pellet washed with $70 \%$ ethanol and air dried for $1 \mathrm{~h}$. To dissolve the pellet, $100 \mu \mathrm{L}$ of TE-buffer $\quad\left(10 \mathrm{mmol} / \mathrm{L}\right.$ Tris- $\mathrm{HCl}$ and $1 \mathrm{mmol} / \mathrm{L}$ EDTA diluted in $\mathrm{H}_{2} \mathrm{O} ; \mathrm{pH}$ 7.5) was added and incubated for $30 \mathrm{~min}$ at $65^{\circ} \mathrm{C}$.

\subsubsection{Polymerase chain reaction (PCR)}

The PCR was carried out to determine either the genotype of the Mcph1 constitutive and conditional knockout mice or the presence of the nestin-Cre transgene using DNA extracted from mouse tail biopsies. The reaction mixture was as followed: $100 \mathrm{ng}$ to $200 \mathrm{ng}$ DNA, $0.4 \mu \mathrm{mol} / \mathrm{L}$ of each primer, $100 \mathrm{mmol} / \mathrm{L}$ dNTP Mix (Bioline), $10 \%$ GB-buffer (166 mmol/L $\left(\mathrm{NH}_{4}\right)_{2} \mathrm{SO}_{4}, 670 \mathrm{mmol} / \mathrm{L}$ Tris-HCl, $67 \mathrm{mmol} / \mathrm{L} \quad \mathrm{MgCl}_{2}, 67 \mu \mathrm{mol} / \mathrm{L}$ EDTA, $50 \mathrm{mmol} / \mathrm{L}$ $\beta$-mercaptoethanol), $10 \%$ DMSO, 2 units BIOTAQ DNA Polymerase (Bioline) diluted in $\mathrm{H}_{2} \mathrm{O}$ in a total volume of $25 \mu \mathrm{L}$. The reaction conditions were:

1. Initial denaturation at $94^{\circ} \mathrm{C}$ for $3 \mathrm{~min}$

2. Denaturation at $94{ }^{\circ} \mathrm{C}$ for $30 \mathrm{~s}$

3.a Primer annealing for Mcph1 gene at $62{ }^{\circ} \mathrm{C}$ for $30 \mathrm{~s}$

3.b Primer annealing for nestin-Cre transgene at $58.5^{\circ} \mathrm{C}$ for $30 \mathrm{~s}$

4. Elongation at $65^{\circ} \mathrm{C}$ for $70 \mathrm{~s}$

5. Final elongation at $65^{\circ} \mathrm{C}$ for $7 \mathrm{~min}$

6. Hold at $4{ }^{\circ} \mathrm{C}$

The steps number 2 to 4 were performed in 35 consecutive cycles.

The following primers were used for the Mcph1 gene:

Mcph1-I5-s: 5'-AGATCTCATTGCAGGTGGTTG-3'

Mcph1-15-as: $\quad$ 5'-GCTGTGGATAACCTGAAGCA-3'

Mcph1-neo-as: $\quad$ 5'-AGGAGCAAAGCTGCTATTGG-3'

Mcph1-I3-s: 5'-TATGATGTCTCAGGTAGCAGCAG-3' 
The primer combination 15-s, 15-as detect the Mcph1 wildtype allele (band of $269 \mathrm{bp}$ ) and the Mcph1 floxed allele (band of $384 \mathrm{bp}$ ). The primers 15-s and neo-as detect the Mcph1 targeted allele (band of 599 bp). The Mcph1 deleted allele can be detected by the primers I3-s and 15-as (band of $900 \mathrm{bp}$ ).

The following primers were used for the nestin-Cre transgene:

Cre1:

5'-CGGTCGATGCAACGAGTGATG-3'

Cre2:

5'-CCAGAGACGGAAATCCATCGC-3'

Actin-B2-1:

5'-CACCGGAGAATGGGAAGCCGAA-3'

Actin-B2-2:

5'-TCCACACAGATGGAGCGTCCAG-3'

The primer combination Cre1, Cre2 detect the nestin-Cre transgene (band of $643 \mathrm{bp}$ ) and the The primers Actin-B2-1 and Actin-B2-2 detect the actin gene (band of $294 \mathrm{bp}$ ) and were used as control.

\subsubsection{Construction of short hairpin RNA (shRNA)-vectors}

For the construction of shRNA-vectors, oligonucleotides targeting the coding sequence and their complementary sequences were inserted into a plasmid containing the U6-promoter. Additionally, the plasmid expresses Egpf, encoding enhanced green fluorescence protein (EGFP) under control of CMV-promoter. All used oligonucleotides contained the hairpin loop sequence: 5'-TTCAAGAGA-3'.

The targeting sequences were:

Luciferase control:

Chk1:

Cdc25b:
5'-GGCTTGCCAGCAACTTACA-3'

5'-GGAAGAAGAGTTGTATGAATC-3'

5'-GGCCCGTGTCTTTCGCTCA-3' 


\subsection{Mouse embryonic fibroblast (MEF) culture and methods}

\subsubsection{Isolation and culture conditions of MEFs}

Matings of female and male mice were set up and females were checked for vaginal plugs. The day on mice were identified with a positive plug was designated as embryonic stage 0.5 (E0.5). Pregnant mice at E13.5 were sacrificed by cervical dislocation and the abdominal wall was opened after cleaning with $70 \%$ ethanol. The peritoneum was opened with sterile scissors and forceps and the organs and fat tissue were moved to expose the uterus. The complete uterus was removed and transferred to $10 \mathrm{~cm}$ Petri dish containing ice cold sterile PBS. The embryos were removed by opening the uterus and collected in separate dishes with ice cold PBS. The embryonic liver was removed and collected for DNA isolation and PCRgenotyping to determine the genotype of the embryos. For the isolation of MEFs the embryos were transferred to a sterile tube and cut into small pieces. The chopped tissue was transferred to a $15 \mathrm{~mL}$ Falcon-tube containing $2 \mathrm{~mL}$ trypsin-EDTA-solution (Invitrogen) and incubated for $6 \mathrm{~min}$ at $37{ }^{\circ} \mathrm{C}$ with occasional inversion of the tube. The supernatant was transferred to a $50 \mathrm{~mL}$ Falcon-tube containing $30 \mathrm{~mL}$ MEF medium and additional $2 \mathrm{~mL}$ trypsin-EDTA-solution was added to the chopped tissue. The trypsinization procedure of the tissue was performed three times and the supernatant was collected in the same tube. The solution was then centrifuged at $240 \times \mathrm{g}$ for $5 \mathrm{~min}$ and the pellet was resuspended in $4 \mathrm{~mL}$ MEF medium, transferred to T25 cell culture dish and cultured at $37{ }^{\circ} \mathrm{C}$ with $5 \% \mathrm{CO}_{2}$.

\subsubsection{Immortalization of MEFs}

MEFs were immortalized according to the 3T3-protocol. Primary MEFs were cultured in MEF medium under normal culture conditions and splitted every three days at a ratio of 1:3 until cells were senescent. The senescent MEFs were maintained at the normal culture conditions with medium change every three days until cells started to proliferate again. The 3T3- 
immortalized MEFs were then amplified and aliquots were kept at liquid nitrogen in MEF freezing medium.

\subsubsection{Transfection of immortalized MEFs}

3T3-immortalized MEFs were transfected using Amaxa Nucleofector Kit R - NIH/3T3 (LONZA). MEFs were passaged 2 to 3 days before transfection. Cells were trypsinized and for each reaction $1 \times 10^{6}$ cells were centrifuged at $200 \times \mathrm{g}$ for $10 \mathrm{~min}$. The supernatant was removed and the pellet was resuspended in $100 \mu \mathrm{L}$ Nucleofector Solution R (LONZA) and $5 \mu \mathrm{g}$ of plasmid-DNA was added. The cell suspension was transferred to a cuvette and electroporated using Nucleofector I Device (LONZA). The electroporated MEFs were either transferred to a 6 well cell culture dish containing $1.5 \mathrm{~mL}$ MEF medium and cultured under normal conditions for $48 \mathrm{~h}$ for FACsorting and protein isolation or transferred to $2 \times 24$ well cell culture dishes for immunocytochemistry.

\subsubsection{Immunocytochemistry of transfected MEFs}

Transfected 3T3-immortalized MEFs $\left(1 \times 10^{6}\right.$ cells) were transferred to $2 \times 24$ well cell culture dishes and cultured at high cell density for $48 \mathrm{~h}$ to arrest the MEFs in $\mathrm{G} 0$ by contact inhibition. MEFs were then released from contact inhibition by trypsinization of one 24 well and seeding on $6 \times 24$ wells containing glass coverslips. The MEFs were fixed with $4 \%$ PFA for 7 min at $4{ }^{\circ} \mathrm{C}, 24 \mathrm{~h}$ to $28 \mathrm{~h}$ after the G0-release to analyze cells in mitosis. After fixation, cells were washed three times for $5 \mathrm{~min}$ with PBS. Then, fixed MEFs were incubated with blocking solution (10 \% BSA, $5 \%$ normal goat serum, $0.4 \%$ Triton $\mathrm{X}-100$ in PBS) for $1 \mathrm{~h}$ at RT in a humidified chamber to block unspecific antibody binding and to permeabilize the cells. The blocking solution was removed and the primary antibody, diluted in blocking solution, was added without washing step and incubated overnight at $4^{\circ} \mathrm{C}$ in a humidified chamber. Then, the primary antibody solution was removed and cells were washed three times for 5 min with 
PBS. The solution of fluorochrome-coupled secondary antibodies, diluted in blocking solution, was added and incubated for $2 \mathrm{~h}$ at RT in the dark using a humidified chamber. After removal of the secondary antibody solution, cells were washed three times with PBS for 5 min. The coverslips were mounted on glass slides using a mounting medium which contains 4',6-diamidino-2-phenylindole (DAPI) to stain the nuclei (ProLong Gold, Invitrogen) and stored for short term at $-20^{\circ} \mathrm{C}$.

\subsection{Mouse embryonic neuronal progenitor cell culture and methods}

\subsubsection{Isolation and culture conditions of neuronal progenitor cells}

Pregnant mice at E14.5 were sacrificed and embryos were isolated as described in chapter 2.3.1. For the isolation of neuronal progenitor cells the brains of the embryos were removed from the skull using small forceps and transferred to a sterile Eppendorf-tube. For the characterization of CNS-specific progenitor cells, newborn (P0) were sacrificed by decapitation and the brains were isolated. The cerebellum of PO brains was removed and the cerebrum was transferred to a sterile Eppendorf-tube. In order to determine the genotype of the embryos and P0 mice, tail biopsies were collected for DNA isolation and PCR-genotyping. For the progenitor cell isolation the brain was cut into small pieces. To produce single cell suspension the tissue was then enzymatically digested with $100 \mu \mathrm{L}$ mouse embryonic brain digestion solution at $37{ }^{\circ} \mathrm{C}$ in a water bath for $1 \mathrm{~h}$ with occasional mixing. To inhibit the enzymatic digestion, $300 \mu \mathrm{L}$ ovomuccoid trypsin inhibitor solution was added. After incubation at $37{ }^{\circ} \mathrm{C}$ for $4 \mathrm{~min}$, additional $300 \mu \mathrm{L}$ ovomuccoid trypsin inhibitor solution was added. The mixture was passed through a $1 \mathrm{~mL}$ syringe with $27 \mathrm{G} \mathrm{x} \mathrm{3/4"}(0.4 \times 20 \mathrm{~mm})$ needle for 5 to 7 times until the tissue pieces had been dissociated and then transferred to a $15 \mathrm{~mL}$ Falcon tube containing $5 \mathrm{~mL}$ DMEM-NutMix/F12-GlutaMax (Invitrogen). After centrifugation at $240 \times \mathrm{g}$ for $5 \mathrm{~min}$ at RT, the supernatant was removed and the cell pellet was resuspended in $4 \mathrm{~mL}$ mouse neuronal 
progenitor cell culture medium. Cell suspension was then transferred to a T25 cell culture flask and cultured upright to avoid attachment of the cells to the coated culture dish which would induce cell differentiation. The incubation conditions of the atmosphere were: $37{ }^{\circ} \mathrm{C}$ with $95 \%$ air and $5 \% \mathrm{CO}_{2}$.

To synchronize neuronal progenitor cells a double thymidine block was used. 5 days after isolation, thymidine (Sigma-Aldrich) was added to the cells to a final concentration of $2 \mathrm{mM}$ and incubated for $19 \mathrm{~h}$ at normal culture conditions. Cells were then released by washing out the thymidine and incubated in fresh neuronal progenitor cells for 9 hours at normal culture conditions. Then a second thymidine block was applied by adding thymidine to the culture medium to a final concentration of $2 \mathrm{mM}$ and incubated for additional $16 \mathrm{~h}$. After this incubation, the neurospheres were released by washing out the thymidine and cultured either in suspension under normal culture conditions for immunoblot analysis or dissociated to obtain single cell suspension and allowed to attach to poly-D-lysine (Sigma-Aldrich) coated glass coverslips and cultured under normal conditions for immunocytochemistry analysis.

\subsubsection{Primary neurosphere formation}

For the formation of primary neurospheres, neuronal progenitor cells, isolated from E14.5 embryos or CNS-progenitor cells, isolated from P0 mice, were seeded directly after isolation at the cell density of $2.0 \times 10^{5}$ cells $/ \mathrm{mL}$ in a total volume of $4 \mathrm{~mL}$ neuronal progenitor cell culture medium. Cells were incubated for 7 days at $37{ }^{\circ} \mathrm{C}$ with $95 \%$ air and $5 \% \mathrm{CO}_{2}$ to form primary neurospheres. The number of neurospheres $/ \mathrm{mL}$ was determined by transferring $0.5 \mathrm{~mL}$ of the cell suspension to one 6 well cell culture dish containing $1.5 \mathrm{~mL}$ of neuronal progenitor cell medium and counting of the neurospheres was performed in duplicates. To measure the neurosphere size, a minimum number of 100 neurospheres was picked and collected in a fresh Eppendorf-tube. $100 \mu \mathrm{L}$ trypsin-EDTA solution (Invitrogen) was added and incubated at $37^{\circ} \mathrm{C}$ in a water bath for $7 \mathrm{~min}$. To inactivate the trypsin activity a mixture of $800 \mu \mathrm{L}$ DMEMNutMix/F12-GlutaMax (Invitrogen) and $50 \mu \mathrm{L}$ FCS (LONZA) was added. To dissociate the neurospheres, the mixture was passed through a $1 \mathrm{~mL}$ syringe with $27 \mathrm{G} \times 3 / 4 "(0.4 \times 20 \mathrm{~mm})$ 
needle for 5 to 7 times followed by the use of a $30 \mathrm{G} \times 1 / 2 "(0.3 \times 13 \mathrm{~mm})$ needle. The total cell number was then measured using a Coulter Counter Z2 (Beckman Coulter).

\subsubsection{Determination of self-renewal capacity of neuronal progenitor cells}

The self-renewal capacity of the neuronal progenitor cells was determined by dissociating the primary neurospheres and replating to form secondary neurospheres. Primary neurospheres were transferred to $15 \mathrm{~mL}$ Falcon tube and centrifuged at $240 \times \mathrm{g}$ for $5 \mathrm{~min}$ at RT. The cell pellet was resuspended in $100 \mu \mathrm{L}$ trypsin-EDTA solution (Invitrogen) and incubated at $37{ }^{\circ} \mathrm{C}$ in

a water bath for $7 \mathrm{~min}$ followed by inhibition of the trypsin by adding $50 \mu \mathrm{L}$ FCS (LONZA), diluted in $800 \mu \mathrm{L}$ DMEM-NutMix/F12-GlutaMax (Invitrogen). In order to obtain single cell suspension, the mixture was passed through a $1 \mathrm{~mL}$ syringe with $27 \mathrm{G} \times 3 / 4$ " $(0.4 \times 20 \mathrm{~mm})$ needle for 5 to 7 times followed by the use of a $30 \mathrm{G} \times 1 / 2 "(0.3 \times 13 \mathrm{~mm})$ needle. After determining the cell number using a Coulter Counter Z2 (Beckman Coulter), the cell density was adjusted to $1.6 \times 10^{5}$ cells $/ \mathrm{mL}$ in a volume of $4 \mathrm{~mL}$ neuronal progenitor culture medium and then cultured at $37{ }^{\circ} \mathrm{C}$ with $95 \%$ air and $5 \% \mathrm{CO}_{2}$ to form secondary neurospheres. After 7 days in culture the measuring of the number and size of the secondary neurospheres was performed as for the counting of the primary neurospheres (see chapter 2.4.2). The steps of dissociating and replating were repeated in order to obtain tertiary neurospheres.

\subsubsection{Immunocytochemistry of neuronal progenitor cells}

On glass coverslips attached neuronal progenitor cells were fixed with $4 \%$ PFA-solution for 7 min at $4{ }^{\circ} \mathrm{C}$ followed by three washing steps with PBS for 5 min each. For short-term storage the fixed cells were kept at $4^{\circ} \mathrm{C}$ in PBS. To block unspecific binding and to permeabilize the cells the fixed neuronal progenitor cells were incubated with blocking solution (10\% BSA, $5 \%$ normal goat serum, $0.4 \%$ Triton X-100 in PBS) for $1 \mathrm{~h}$ at RT in a humidified chamber. The blocking solution was removed and the primary antibody, diluted in blocking solution, was 
added without washing step and incubated overnight at $4^{\circ} \mathrm{C}$ in a humidified chamber. The next day the primary antibody solution was removed and cells were washed three times with PBS for $5 \mathrm{~min}$ each followed by incubation with fluorochrome-coupled secondary antibodies, diluted in blocking solution, for $2 \mathrm{~h}$ at RT in the dark using a humidified chamber. After removal of the secondary antibody solution, cells were washed three times with PBS for 5 min each. The coverslips were mounted on a glass slide using a mounting medium which contains (DAPI) to counterstain the nuclei (ProLong Gold, Invitrogen) and stored at $-20^{\circ} \mathrm{C}$.

\subsubsection{Metaphase chromosome preparation}

For the cytogenetic analysis, primary neurospheres on DIV7 after isolation were incubated under normal culture conditions for $7 \mathrm{~h}$ with fresh neuronal progenitor cell culture medium containing $1 \mu \mathrm{g} / \mathrm{mL}$ colcemid (N-deacetyl-N-methylcolchicine; Invitrogen) to induce metaphase arrest. Cells were then transferred to a $15 \mathrm{~mL}$ Falcon tube and centrifuged at $130 \times \mathrm{g}$ for $8 \mathrm{~min}$. The supernatant was removed and cells were washed with $10 \mathrm{~mL}$ PBS. The centrifugation step was repeated and after removal of the supernatant, $2 \mathrm{~mL}$ trypsin-EDTA solution (Invitrogen) was added to the cell pellet and incubated at $37{ }^{\circ} \mathrm{C}$ for $5 \mathrm{~min}$. To inactivate the trypsin, $10 \mathrm{~mL}$ DMEM-NutMix/F12-GlutaMax (Invitrogen) and $500 \mu \mathrm{L}$ FCS (LONZA) was added. The cells were centrifuged at $130 \mathrm{xg}$ for $8 \mathrm{~min}$ and after removal of the supernatant the cell pellet was resuspended in $2.5 \mathrm{~mL}$ hypotonic solution $(0.03 \mathrm{~mol} / \mathrm{L}$ sodium citrate) and incubated at $37^{\circ} \mathrm{C}$ for $15 \mathrm{~min}$. Freshly prepared fixative solution (methanol and $37 \%$ acetic acid at a ratio of $3: 1$ ) was then added and incubated on ice for $15 \mathrm{~min}$. The supernatant was removed after centrifugation (130 x g, $8 \mathrm{~min}$ ) except for $1 \mathrm{~mL}$ which remained in the tube. While mixing, $10 \mathrm{~mL}$ fresh fixative solution was added and again centrifuged (130 $x \mathrm{~g}, 8 \mathrm{~min}$ ), the supernatant removed except for $1 \mathrm{~mL}$ and cells were resusupended in $10 \mathrm{~mL}$ fresh fixative solution. The steps of centrifugation and resuspension in fresh fixative solution were repeated at least three times. After the final resuspension in $10 \mathrm{~mL}$ fixative solution, the solution was dropped onto glass slides and air dried at RT for at least $10 \mathrm{~min}$. The slides were mounted with glass coverslips using DAPI-containing mounting medium (ProLong Gold, Invitrogen) and stored for at $-20^{\circ} \mathrm{C}$. 


\subsubsection{Pair cell assay}

The Pair cell assay was initially developed by Shen et al. to follow the cell division of single neuronal progenitor cells in vitro with subsequent identification of protein distribution in the two daughter cells (paired cells) by antibody staining (Shen et al., 2002). In the current study the Pair Cell assay was modified in order to identify the cell fate (progenitor or neuron) of two daughter cells after following the division of a single neuronal progenitor cell. Neuronal progenitor cells were isolated from E14.5 embryos and cultured under normal culture conditions to form primary neurospheres. On DIV7, neurospheres were dissociated to obtain single cell suspension. The cells were then plated on poly-D-lysine coated glass coverslips which were placed in 24 well cell culture dishes at clonal density (10,000 cells per $12 \mathrm{~mm}$ glass coverslip) which would allow the identification of daughter cells (pair cells) of a single neuronal progenitor cell. Subsequently cells were incubated for $24 \mathrm{~h}$ in a culture medium (Neurobasal medium (Invitrogen) supplemented with B-27 (Invitrogen), $2 \mathrm{mmol} / \mathrm{L}$ L-glutamine (Invitrogen) and $10 \mathrm{ng} / \mathrm{mL}$ bFGF (Peprotech)) that allows the specific differentiation of neuronal progenitor cells into neurons. Single cells were identified $2 \mathrm{~h}$ after plating using a Microscope and cells in close proximity were marked using a pen on the culture dish and excluded from the analysis. After the incubation, cells were fixed with $4 \%$ PFA at $4{ }^{\circ} \mathrm{C}$ for 7 min and then washed three times with PBS for 5 min each. After the last washing step, the antibody-staining was performed as described in chapter 2.4.4. The Antibodies were: mouse anti-Nestin and rabbit anti- $\beta$-tubulinlll to label neuronal progenitor cells and neurons, respectively. The paired cells were quantified and then grouped into three different types of division modes: i) progenitor/progenitor ( $\mathrm{P} / \mathrm{P}$; both daughter cells are nestin-positive);

ii) neuron/neuron ( $\mathrm{N} / \mathrm{N}$; both daughter cells are positive for $\beta$-tubulinlll and iii) progenitor/neuron $(\mathrm{P} / \mathrm{N}$; one daughter cell is nestin-positive and one daughter cell is positive for $\beta$-tubulinIII. 


\subsection{Protein analysis}

\subsubsection{Whole cell protein isolation}

Transfected MEFs with EGFP-shRNA-vectors were trypsinized $48 \mathrm{~h}$ after transfection and centrifuged at $240 \times \mathrm{g}$. The supernatant was removed and the pellet from one 6 well cell culture dish was resuspended in $2 \mathrm{~mL}$ PBS containing $2 \%$ FCS. The GFP-positive cells were then purified from the cell suspension using Fluorescence activated cell sorting (FACS; FACSAria cell sorter, Becton Dickinson). Sorted GFP-positive MEFs, harvested untransfected MEFs or synchronized primary neurospheres were centrifuged at $240 \times \mathrm{g}$ for $5 \mathrm{~min}$. The supernatant was removed and the cell pellet was washed with PBS followed by an additional centrifugation step at the same conditions. After removal of the supernatant, the cell pellet was resuspended in $50 \mu \mathrm{L}$ cell extraction buffer $(50 \mathrm{mM}$ Tris- $\mathrm{HCl}, 150 \mathrm{mM} \mathrm{NaCl}, 1 \mathrm{mM}$ EDTA, $0.25 \%$ Na-deoxycholate, $1 \mathrm{mM} \mathrm{Na}_{3} \mathrm{VO}_{4}, 10 \mathrm{mM} \mathrm{NaF}, 0.1 \mathrm{mM}$ phenylmethylsulfonylfluorid, $1 \%$ NP40, 1 x Roche Complete Protease Inhibitor Cocktail) and incubated for 30 min on ice. Then centrifuged at $15,700 \times \mathrm{g}$ for $10 \mathrm{~min}$ at $4{ }^{\circ} \mathrm{C}$ and the supernatant was transferred to a new reaction tube and stored at $-80^{\circ} \mathrm{C}$. Bradford assay was used to determine the protein concentration. $2 \mu \mathrm{L}$ of the protein extract were mixed with $200 \mu \mathrm{L}$ Bradford solution (BIO-RAD Laboratories) and $798 \mu \mathrm{L} \mathrm{H}_{2} \mathrm{O}$ and the absorbance at $595 \mathrm{~nm}$ was determined with a spectrometer (Eppendorf BioPhotometer).

\subsubsection{Electrophoretic protein separation}

For the electrophoretic separation by SDS-polyacrylamide gel electrophoresis (SDS-PAGE), the $20 \mathrm{~mL}$ of protein extracts were denaturated with $5 \mathrm{~mL}$ sodium dodecyl sulfate (SDS)sample buffer $(0.33 \mathrm{mM}$ Tris- $\mathrm{HCl}, 25 \%$ glycerol, $6 \mathrm{~g}$ SDS, $0.93 \mathrm{~g}$ Dithiothreitol, $1.2 \mathrm{mg}$ bromphenol blue in $12 \mathrm{~mL} \mathrm{H}_{2} \mathrm{O}$ ) at $95{ }^{\circ} \mathrm{C}$ for $10 \mathrm{~min}$ followed by cooling on ice for several minutes and loading on a SDS gel. The SDS gel consisted of a stacking gel (5\% acryl amide, $0.125 \mathrm{mM}$ Tris- $\mathrm{HCl} \mathrm{pH} 6.8,0.1 \%$ SDS, $0.1 \%$ ammonium persulfate, $0.1 \%$ Tetra-methyl- 
ethylene-diamine (TEMED) in $\mathrm{H}_{2} \mathrm{O}$ ) and a resolving gel (10\% acryl amid, $0.375 \mathrm{mM}$ Tris- $\mathrm{HCL}$ $\mathrm{pH}$ 8.8, $0.1 \%$ SDS, $0.1 \%$ ammonium persulfate, $0.01 \%$ TEMED in $\mathrm{H}_{2} \mathrm{O}$ ). To estimate the protein size, $10 \mathrm{~mL}$ of protein marker (ProSieve Color Protein Marker, Lonza) was loaded on the gel. The electrophoresis was performed in Running buffer $(200 \mathrm{mM}$ glycine, $25 \mathrm{mM}$ Tris, $0.1 \% \mathrm{SDS}$ in $\mathrm{H}_{2} \mathrm{O}$ ) at $80 \mathrm{~V}$ until the samples reached the resolving gel and then at $120 \mathrm{~V}$ until samples reached the lower border of the gel.

\subsubsection{Immunoblot analysis}

At the end of electrophoretic protein separation, the resolving gel was equilibrated in Transfer buffer (200 mM glycine, $25 \mathrm{mM}$ Tris, $20 \%$ methanol in $\mathrm{H}_{2} \mathrm{O}$ ) for $10 \mathrm{~min}$. At the same time, a polyvinylidene fluoride (PVDF)-membrane (BIO-RAD Laboratories) was equilibrated in methanol for $10 \mathrm{~min}$. Then, the PVDF-membrane was transferred on the gel and transfer cassette was assembled with Whatman-papers on both sides. The transfer was performed in Transfer buffer at $30 \mathrm{~V}$ overnight at $4{ }^{\circ} \mathrm{C}$. Next day, the PVDF-membrane was removed, rinsed 3 times and washed for $5 \mathrm{~min}$ in TBS-T (20 mM Tris, $150 \mathrm{mM} \mathrm{NaCl}, 0.1 \%$ Tween20 in $\mathrm{H}_{2} \mathrm{O}$; $\mathrm{pH}$ 7.4). The PVDF-membrane was then blocked with blocking solution (either $5 \%$ non-fat dried milk in TBS-T or $5 \%$ BSA in TBS-T) for $1 \mathrm{~h}$ with agitation. After removal of the blocking solution, the PVDF-membrane was incubated with the primary antibody, diluted in blocking solution, at $4{ }^{\circ} \mathrm{C}$ overnight with agitation. Next day, the membrane was washed three times for 10 min with TBS-T with agitation. The HRP-linked secondary antibody was diluted in blocking solution and added to the membrane followed by incubation for $1 \mathrm{~h}$ with agitation. Then, the secondary antibody was removed and the membrane was washed three times for 10 min with TBS-T with agitation. For the detection, an enhanced chemiluminescence kit was used (ECL, Amersham) and the membrane was exposed to an x-ray film (Amersham). 


\subsection{Histological techniques}

\subsubsection{Isolation and fixation of embryonic and postnatal mouse brains}

Pregnant mice at embryonic days E13.5, E14.5, E15.5, E17.5 or E18.5 were sacrificed by cervical dislocation and embryos were isolated as described in chapter 2.3.1. Tail biopsies of embryos were collected for DNA isolation and genotyping by PCR. Embryos at and before embryonic stage E15.5 were decapitated and the complete head was immersion fixed overnight with $4 \%$ PFA at $4{ }^{\circ} \mathrm{C}$. Embryos at later stage than E15.5 and newborn mice (postnatal day 0, P0) were sacrificed by decapitation and brains were removed with forceps and small scissors with the help of a stereo microscope (Zeiss Stemi 2000) and then fixed overnight with $4 \%$ PFA at $4{ }^{\circ} \mathrm{C}$. Adult mice were sacrificed by $\mathrm{CO}_{2}$ asphyxiation and brains were removed using forceps and scissors followed by fixation with $4 \%$ PFA at $4{ }^{\circ} \mathrm{C}$ for 2 days. The further procedure depended on the type of histological method (see chapter 2.6.2 and 2.6.3).

\subsubsection{Preparation of paraffin embedded mouse brain sections}

After fixation with $4 \%$ PFA, the isolated brains were washed briefly with PBS and then washed with $30 \%$ ethanol for at least $30 \mathrm{~min}$. The brains were then transferred to $50 \%$ ethanol for short term storage. For the embedding in paraffin, an automatic device (Shandon Excelsior) was used to apply a series of ascending ethanol (70\% for $30 \mathrm{~min}$, two times $95 \%$ for $20 \mathrm{~min}$ each, three times $100 \%$ for 30 min each) and then three steps of xylene for 30 min before transferring to liquid paraffin at $60{ }^{\circ} \mathrm{C}$. The brains were washed three times with paraffin at $60{ }^{\circ} \mathrm{C}$ for at least $30 \mathrm{~min}$ each. This was followed by placing the brains into metallic moulds with the desired orientation, either sagittal or coronal, and then liquid paraffin at $60{ }^{\circ} \mathrm{C}$ was poured in the moulds. The moulds containing the brains in liquid paraffin were placed on a cooling plate to achieve a fast and constant cooling of the paraffin. The paraffin embedded brains were then sectioned using a microtom (Microm HM355S) at a thickness of $5 \mu \mathrm{m}$ and 
sections were mounted on glass slides and allowed to dry overnight at $55^{\circ} \mathrm{C}$. Until usage, the slides were stored at a dry place at RT.

\subsubsection{Preparation of cryosections of mouse brains}

For the preparation of cryosections, the fixed brains were removed from the $4 \%$ PFA-solution and washed briefly with sterile PBS. To protect the tissue from damage by ice crystals during the freezing procedure, the brains were transferred to a solution of $30 \%$ sucrose in sterile PBS and incubated for 2 to 4 days at $4{ }^{\circ} \mathrm{C}$. Brains were incubated in the sucrose-solution until the tissue sunk which indicated that the sucrose was absorbed completely by the tissue. The brains were embedded in a plastic mould using a cryomatrix (Neg -50, Richard-Allan Scientific) and snap frozen in liquid nitrogen $\left(-196{ }^{\circ} \mathrm{C}\right)$. The brains were then stored at $-80{ }^{\circ} \mathrm{C}$ for at least $24 \mathrm{~h}$. For the sectioning, the frozen brains were transferred to a cryostat (Microm HM550) which was set to $-19{ }^{\circ} \mathrm{C}$ and allowed to warm up for $30 \mathrm{~min}$. The sections were prepared at a thickness of 12 to $20 \mu \mathrm{m}$ and mounted on a glass slide. After brief drying at RT, sections were stored at $-80^{\circ} \mathrm{C}$ before use.

\subsubsection{Hematoxylin and Eosin (H\&E)-staining}

H\&E staining is a widely used method in histology which gives information about the general structure of tissue sections. The oxidation product of hematoxylin is haematin which binds to lysine residues of nuclear histones, thereby staining the cell nuclei blue. To obtain more cellular details, the tissue is counterstained with eosin, which stains proteins in red colour. In this analysis the H\&E staining was performed only on paraffin sections. 
The staining procedure was as follows:

1. Deparaffination:

$\begin{array}{ll}2 \times 10 \text { min } & \text { xylene } \\ 3 \text { min } & 100 \% \text { ethanol } \\ 1 \mathrm{~min} & 100 \% \text { ethanol } \\ 1 \mathrm{~min} & 95 \% \text { ethanol } \\ 1 \mathrm{~min} & 70 \% \text { ethanol } \\ 1 \mathrm{~min} & 50 \% \text { ethanol } \\ 2 \mathrm{~min} & \mathrm{H}_{2} \mathrm{O}\end{array}$

2. Staining:

$\begin{array}{ll}1 \text { min } & \text { hematoxylin } \\ 1 \text { min } & \mathrm{H}_{2} \mathrm{O} \\ 4 \text { min } & \mathrm{H}_{2} \mathrm{O} \\ 1 \text { min } 30 \mathrm{~s} & \text { eosin } \\ 15 \mathrm{~s} & \mathrm{H} 2 \mathrm{O}\end{array}$

3. Dehydration:

$\begin{array}{ll}30 \mathrm{~s} & 50 \% \text { ethanol } \\ 1 \mathrm{~min} & 70 \% \text { ethanol } \\ 2 \times 30 \mathrm{~s} & 95 \% \text { ethanol } \\ 2 \times 2 \mathrm{~min} & 100 \% \text { ethanol } \\ 2 \times 2 \mathrm{~min} & \text { xylene }\end{array}$

After the staining and dehydration, the slides were mounted with coverslips and a xylenebased mounting medium (Thermo Scientific). Mounted slides were dried overnight at RT before analysis and stored at RT. 


\subsubsection{Immunohistochemistry}

For the immunohistochemical analysis of mouse brain sections either paraffin embedded sections or cryosections were used. Paraffin sections were deparaffinized as described in chapter 2.6.4. Cryosections were taken out from $-80{ }^{\circ} \mathrm{C}$ and allowed to air dry at RT for $10 \mathrm{~min}$ with subsequent washing with PBS for 15 min before staining. The following steps were equally performed with paraffin embedded sections and cryosections. For the antigen retrieval, the sections were transferred to $10 \mathrm{mM}$ sodium citrate $(\mathrm{pH} \mathrm{6.0)}$ and heated with a microwave at $800 \mathrm{~W}$ until boiling and then kept at a sub-boiling temperature for $10 \mathrm{~min}$ with occasional heating. The sections were cooled down for $30 \mathrm{~min}$ at RT in the same solution followed by three washing steps for 5 min each with PBS. To reduce unspecific antibody binding, the sections were incubated with blocking solution (1\% BSA, $5 \%$ normal goat serum and $0.4 \%$ Triton $\mathrm{X}-100$ in PBS) for $1 \mathrm{~h}$ at RT in a humidified chamber. The blocking solution was then removed and the primary antibodies, diluted in blocking solution, were applied onto the sections and incubated overnight at $4{ }^{\circ} \mathrm{C}$ in a humidified chamber. The next day, sections were washed with PBS for three times 5 min each and then the fluorochrome-coupled secondary antibodies, which were diluted in blocking solution, were applied to the sections and incubated for $2 \mathrm{~h}$ at RT in the dark in a humidified chamber. Sections were then washed for three times 5 min each with PBS and then mounted with coverslips and a DAPI-containing mounting medium (ProLong Gold, Invitrogen) and allowed to air dry for several hours. Stained sections were stored for short term in a dark box at $-20^{\circ} \mathrm{C}$.

\subsubsection{In vivo bromodeoxyuridine (BrdU)-proliferation assay}

The in vivo BrdU-proliferation assay was used to determine the proliferation rate of neuronal progenitor cells during mouse neurogenesis. An injection of BrdU-solution into pregnant mice leads to the incorporation of the thymidine analogue BrdU into the DNA of replicating cells. The BrdU-incorporated cells can be detected on mouse embryonic brain section after fixation using immunohistochemistry. Pregnant mice were injected intraperitoneal with a single dose of $\mathrm{BrdU}$ at $50 \mu \mathrm{g} / \mathrm{g}$ body weight. $1 \mathrm{~h}$ after injection the pregnant mother was sacrificed, mouse 
embryos removed and heads of the embryos were perfusion fixed overnight with $4 \%$ PFA at $4{ }^{\circ} \mathrm{C}$. Paraffin embedded sections of the embryonic heads were prepared as described in chapter 2.6.2. After deparaffination, the sections were transferred to $10 \mathrm{mmol} / \mathrm{L}$ sodium citrate $(\mathrm{pH} \mathrm{6.0)}$ and heated until boiling temperature with a microwave (800 W) and then kept at a sub-boiling temperature for $10 \mathrm{~min}$. The sections were cooled down for $30 \mathrm{~min}$ at RT in the same solution and then washed with PBS for three times 5 min each. Subsequently, the sections were incubated with $2 \mathrm{~mol} / \mathrm{L} \mathrm{HCl}$ at $37{ }^{\circ} \mathrm{C}$ for $30 \mathrm{~min}$ to denaturate the DNA. This was followed by three washing steps for 5 min with PBS and then sections were incubated with trypsin solution (Vector Laboratories) at $37^{\circ} \mathrm{C}$ for $20 \mathrm{~min}$. After additional three washing steps, sections were blocked with blocking solution (1 \% BSA, $5 \%$ normal goat serum and $0.4 \%$ Triton X-100 in PBS) for $1 \mathrm{~h}$ at RT in a humidified chamber. The antibody-staining with a rat BrdU-antibody was performed as described in chapter 2.6.6.

\subsubsection{Cell cycle exit index}

Neuronal progenitor cells in S-phase were pulse labeled in vivo with a single dose of BrdU and then incubated for $24 \mathrm{~h}$. A double antibody-labeling against BrdU and the cell cycle marker Ki76 allows the determination of cells exited the cell cycle during $24 \mathrm{~h}$. Pregnant mice at embryonic day E14.5 were injected intraperitoneal with a single dose of BrdU at $50 \mu \mathrm{g} / \mathrm{g}$ body weight and incubated for $24 \mathrm{~h}$. Then, the pregnant mother was sacrificed and mouse embryos at embryonic stage E15.5 were removed and heads of the embryos were perfusion fixed overnight with $4 \%$ PFA at $4{ }^{\circ} \mathrm{C}$. Paraffin embedded sections of the embryonic heads were prepared (see chapter 2.6.2) and the BrdU-staining procedure was performed as described in chapters 2.6.6 and 2.6.7. In addition to the rat anti-BrdU antibody, the rabbit anti-Ki67 antibody was added to the primary antibody solution. The double staining procedure revealed three different types of labeled cells: i) single BrdU-positive cells are those cells which have been in S-phase at the time of BrdU-injection, but exited the cell cycle (G0), ii) BrdU-Ki67-double positive cells have been in S-phase at the time of injection and are still in the cell cycle and iii) single Ki67-positive cells are those cells which are in the cell cycle, which have not been in S-phase at the time of injection. The cell cycle exit index was calculated as a number of cells 
that exited the cell cycle (BrdU-positive, Ki67-negative) as a percentage of the total BrdUpositive cells (Ki67-positive and Ki67-negative).

\subsubsection{Terminal deoxynucleotidyl transferase dUTP nick end labeling (TUNEL) on mouse brain sections}

TUNEL was carried out to detect apoptotic cells in embryonic or adult brain sections. The method detects fragmented DNA which is a hallmark of apoptosis at late stage. The enzyme terminal deoxynucleotidyl transferase catalyzes an addition of dUTP, which can be biotinlinked, to 3'-hydroxyl ends of DNA single- or double-strand breaks. Such biotin-labeled DNA breaks can be detected using the biotin-binding molecule streptavidin, which is linked to a fluorescence dye. Cryosections of adult brains or embryonic brains were taken out of $-80{ }^{\circ} \mathrm{C}$ and air dried for $10 \mathrm{~min}$ at RT with subsequent washing with PBS for $15 \mathrm{~min}$. After this, sections were transferred to $10 \mathrm{mmol} / \mathrm{L}$ sodium citrate buffer $(\mathrm{pH} \mathrm{6.0)}$ and incubated for $10 \mathrm{~min}$ at sub-boiling temperature and then allowed to cool down to RT for $30 \mathrm{~min}$ in the same buffer. The sections were washed three times 5 min each with PBS with subsequent applying of the reaction mixture onto the sections and incubation at $37{ }^{\circ} \mathrm{C}$ for $1 \mathrm{~h}$ in a humidified chamber. The reaction mixture (1x Amersham Buffer, 0.3 units/ $\mu \mathrm{L}$ Amersham terminal transferase, $6.66 \mu \mathrm{mol} / \mathrm{L}$ biotin-linked dUTP (Roche Diagnostics), diluted in $\mathrm{H}_{2} \mathrm{O}$ ) was added in a total volume of 20 to $40 \mu \mathrm{L}$, depending on the size of the section. After removal of the reaction mixture, cells were washed for three times $5 \mathrm{~min}$ with PBS. Streptavidin-Cy3 (Sigma-Aldrich) at a dilution of 1:500 in PBS containing $1 \%$ BSA was applied onto the sections and incubated for $1 \mathrm{~h}$ at RT. After this, sections were washed with PBS three times for $5 \mathrm{~min}$ and then mounted with coverslips and a DAPI-containing mounting medium (ProLong Gold, Invitrogen) and stored for short term at $-20^{\circ} \mathrm{C}$. 


\subsection{Mouse behavioral tests}

\subsubsection{Barnes maze test}

The Barnes maze test was developed by Carol Barnes as dry-land maze for spatial learning and memory (Barnes, 1979). The Barnes maze consists of a circular platform (122 cm diameter) with 40 equally spaced holes $(5 \mathrm{~cm}$ diameter) surrounding the perimeter of the maze. The mice were placed in the centre of the maze and received reinforcement (noise) to escape from the open platform to a dark chamber which is placed under one hole referred to as target hole. Visual cues were placed surrounding the maze that the animals could learn the spatial location of the target hole. On the pre-training trial, the mouse was placed in the centre of the maze in a cylindrical start chamber $(10 \mathrm{~cm}$ inner diameter). After $10 \mathrm{~s}$ the noise was applied, the chamber was lifted and the mouse was pre-trained to find the target hole by guiding it. The mouse remained in the target box for 2 min without noise. The first trail started immediately after the pre-training trail. At the beginning of each trail, the mouse was placed in the same start chamber and after $10 \mathrm{~s}$ the noise was applied and the start chamber was lifted. The mouse was allowed to explore the maze. The trail ended when the mouse entered the target box or after 3 min have elapsed. After the trail, the mouse was allowed to stay in the target box for 1 min without noise. During the trail, the number of errors (nose pokes in holes different from the target hole) and the time until the mouse entered the target hole was recorded to evaluate the spatial learning ability. The animals received 4 trails per day with an interval of 15 min between each trail during 4 consecutive days. After each trail, the maze was cleaned to avoid olfactory cues. One day after the last trail, the animals received the probe trail to assess the spatial short-term memory. The target box was removed from the target hole that the animals could not escape from the maze. The mouse was allowed to explore the maze with reinforcement and was removed after $90 \mathrm{~s}$. During the probe trail the number of nose pokes in each hole was recorded to determine if the mouse remembers the location of the target hole. 


\subsubsection{Rotarod test}

The Rotarod test has been developed to assess the effect of drugs on the motor coordination of mice (Dunham and Miya, 1957). In the current study, the Rotarod test was used to evaluate motor coordination defects of the Mcph1-knockout mice. The Rotarod (Ugo Basile) used in the the current study consisted of a rotating roller with $3.5 \mathrm{~cm}$ in diameter and several circular separators placed along the rod to divide the roller into compartments with equal size for testing up to 5 mice simultaneously. The Rotarod is connected to a power source for turning the roller and consists of software which allows pre-programming of session protocols with varying speeds. Mice were trained one day before measurement in one session. On the pretraining trail, mice were placed on the rod and trained at a constant speed of 5 rounds per minute (rpm) for $5 \mathrm{~min}$. The measurement was performed by placing the mice on the rod perpendicular to the axis of rotation and the conditions were: start speed $2 \mathrm{rpm}$ with acceleration of $1 \mathrm{rpm} / 5 \mathrm{~s}$ to a final speed of $50 \mathrm{rpm}$. The persistence time of the mice on the rod was recorded. The measurement was performed on 5 consecutive days.

\subsubsection{Balance bar test}

The balance bar test was performed to test motor coordination abilities of mice. The experimental setup consisted of a bar with a diameter of $1 \mathrm{~cm}$ and the length of $1 \mathrm{~m}$ and was placed at a height of $1 \mathrm{~m}$. Mice were place on one side of the bar and received reinforcement (noise) to walk along the bar to a dark chamber which was placed at the other side of the bar. The number of errors was recorded as hindfoot missteps. Each mouse was tested in 3 trials. 


\section{Results}

\subsection{Deletion of Mcph1 in mice leads to primary microcephaly}

\subsubsection{Reduced brain size and weight of $M c p h 1^{\Delta / \Delta}$ mice at newborn stage and adulthood}

Mutations in the MCPH1 gene can lead to microcephaly in humans, which is present at birth (primary microcephaly; Jackson et al., 2002; Jackson et al., 1998). This suggests a role for $\mathrm{MCPH} 1$ in embryonic brain development. To study the function of MCPH1 in mammalian CNS development, we disrupted the Mcph1 gene in mice, referred to as Mcph1 ${ }^{\Delta / \Delta}$ mice (Sukchev, 2010). Since we did not observe any phenotypic differences between $M c p h 1^{+/+}$and heterozygous deleted $M c p h 1^{+/ \Delta}$ littermates, we grouped mice with these two genotypes into the control group and referred them to as $M c p h 1^{\text {ctr }}$ mice.

We first addressed, whether deletion of Mcph1 in mice would lead to primary microcephaly. Since the clinical definition of primary microcephaly is a reduction of brain size at birth without progression of severity with age, we analyzed the brain size of newborn mice at postnatal day 0 (P0) and of adult mice with the age of 3 months (P90; Figure 9). In newborn mice, we observed a reduced brain size of $M c p h 1^{\Delta / \Delta}$ mice compared to their littermate controls (Figure 9A) and a significantly reduction of the brain weight (Figure 9B). We observed the reduced brain size and weight also in adult $M c p h 1^{\Delta / \Delta}$ mice compared to control mice. No progression in the severity of the microcephaly phenotype was observed (Figure 9C, D). 
A

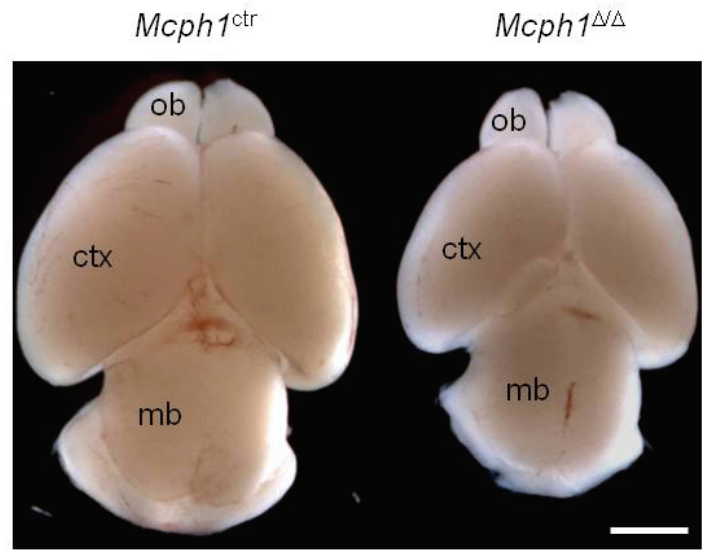

C

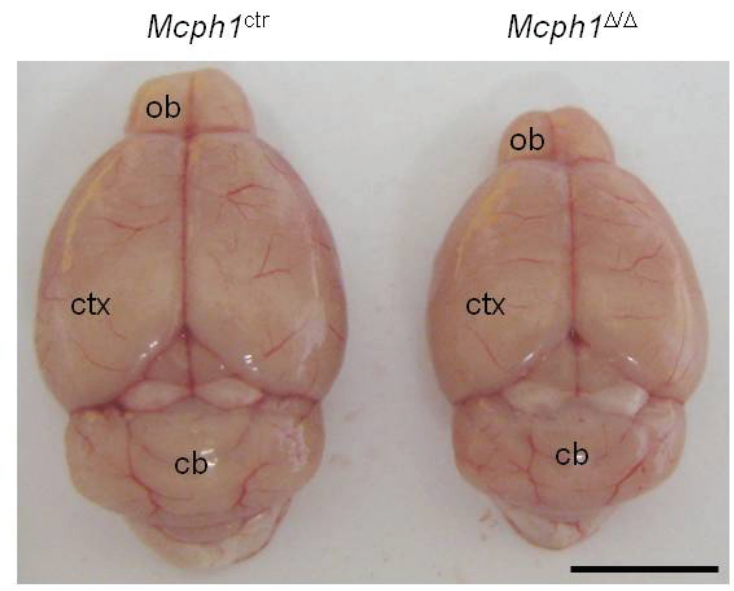

B

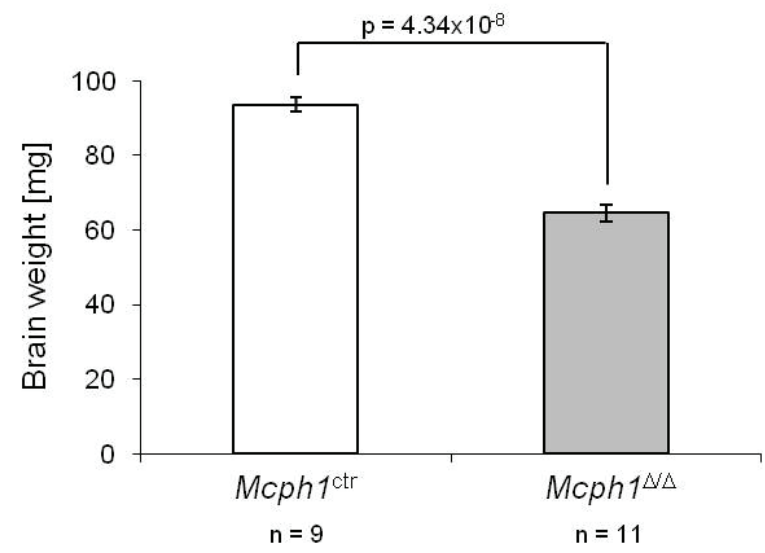

D

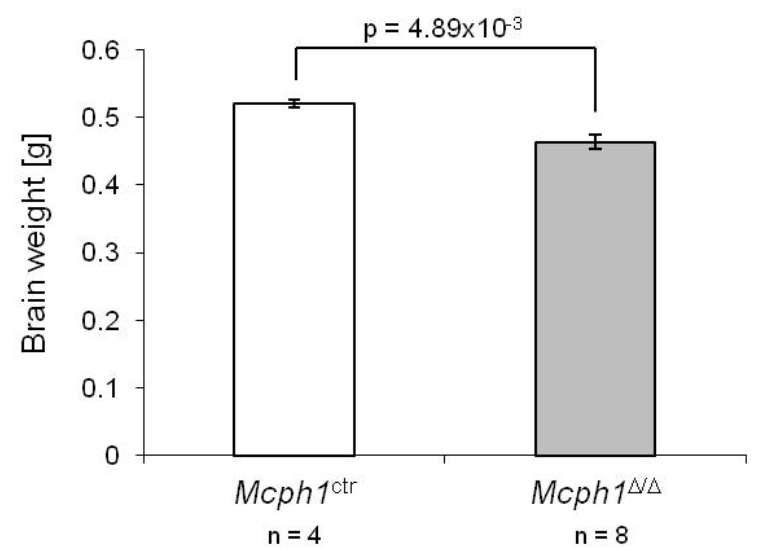

Figure 9: Reduced brain size and weight of $M c p h 1^{\Delta / \Delta}$ newborn (P0) and adult (P90) mice. (A) Caudal view of representative brains isolated from a newborn (P0) $M c p h 1^{\Delta / \Delta}$ mouse and a littermate control. Scale bar: $500 \mathrm{~mm}$; ob = olfactory bulb; ctx = cerebral cortex; $\mathrm{mb}=$ midbrain.

(B) Brain weight of PO mice. Means \pm standard error of means of $n=$ number of mice are shown. Student's t-test was performed for statistical analysis.

(C) Representative image of brains (caudal view) from a 3 months old (P90) Mcph $1^{\Delta / \Delta}$ mouse and littermate control. Scale bar: $5 \mathrm{~mm}$; ob = olfactory bulb; ctx = cerebral cortex; $\mathrm{cb}=$ cerebellum.

(D) Brain weight of P90 mice. Means \pm standard error of means of $n=$ number of mice are shown. Student's t-test was performed for statistical analysis. 


\subsubsection{Reduced cerebral cortical thickness of $M c p h 1^{\Delta / \Delta}$ mice at P0}

To analyze whether there is a general reduction of the brain or certain brain regions are more affected, we performed histology brain sections of newborn mice. Figure 10 shows coronal brain sections of mice at P0 which were stained by hematoxylin and eosin (H\&E) to reveal the morphological structure. $M c p h 1^{\Delta / \Delta}$ mice show a pronounced size reduction of the cerebral cortex (ctx) compared with $M c p h 1^{\text {ctr }}$ mice in both lateral and radial dimensions (Figure 10A). A higher magnification of the indicated areas in Figure 10A (rectangles) shows a general $20 \%$ reduction of the cortical thickness (radial dimension, Figure 10B) The size of other brain regions, hippocampus ( $\mathrm{hp}$ ), midbrain $(\mathrm{mb})$ or amygdala $(\mathrm{am})$, seem less affected in the $M c p h 1^{\Delta / \Delta}$ mice (Figure 10A).

\subsubsection{Conditional CNS-specific deletion of Mcph1 leads to primary microcephaly}

The phenotypic observations suggest that deletion of Mcph1 leads to primary microcephaly in mice. However, the $M c p h 1^{\Delta / \Delta}$ mice in addition show a reduced body weight (Figure 11B) and when we calculated the ratio of brain weight relative to body weight there was no significant reduction of $M c p h 1^{\Delta / \Delta}$ mice compared with control mice (Figure 11C). We therefore asked whether the reduced brain size is a secondary effect due to a general reduction of the body size. To address this question, we deleted Mcph1 specific in the CNS of mice. We generated Mcph1 conditional knockout mice, carrying either targeted or floxed alleles (designated as $M c p h 1^{T / T}$ mice or $M c p h 1^{\text {floxflox }}$, respectively, which allow Cre-mediated tissue-specific gene deletion (Sukchev, 2010). Crossing of $M c p h 1^{T / T}$ or $M c p h 1^{\text {floxflox }}$ mice with the transgenic mouse line nestin-Cre, expressing the Cre transgene, encoding a recombinase under the control of the nestin promoter (Tronche et al., 1999), would lead to the specific deletion of Mcph1 in the CNS (designated as Mcph $1^{\mathrm{CNS} \Delta}$ mice). It was shown that nestin was highly expressed in the neural tube already at E9.5 (Zimmerman et al., 1994). Thus, the $M c p h 1^{\text {CNS } \Delta}$ embryos potentially delete Mcph1 in NEs before the onset of neurogenesis. 
A

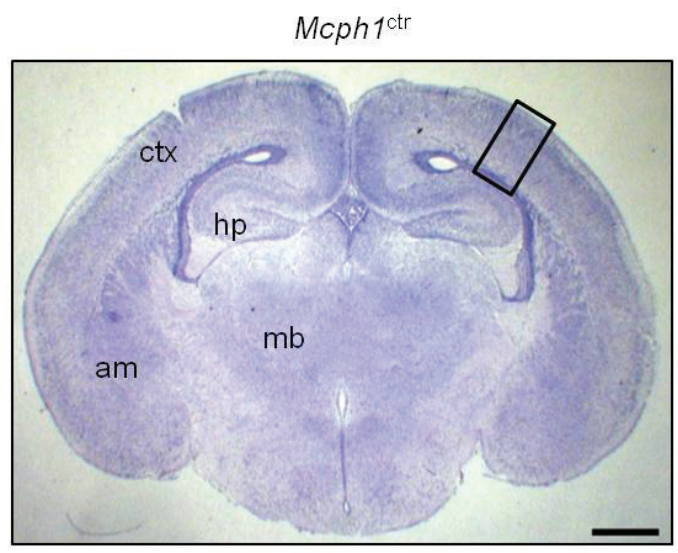

$\operatorname{Mcph} 1^{\Delta \Delta}$

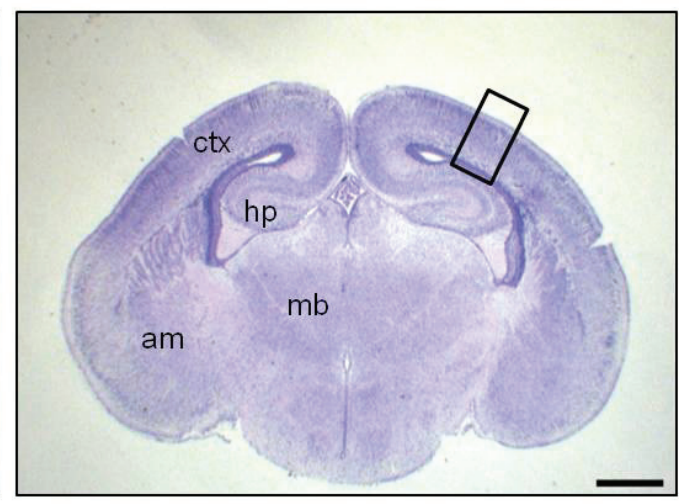

B

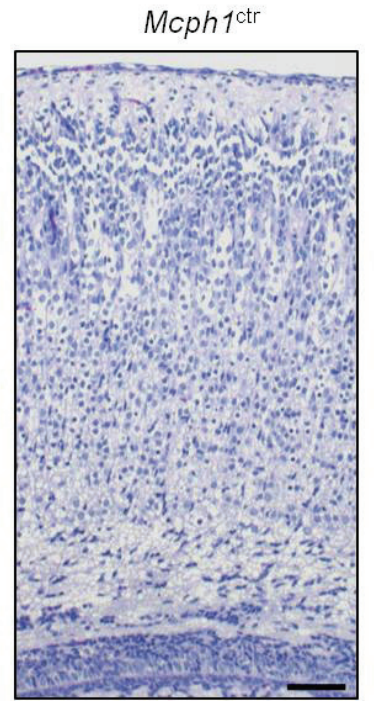

$\operatorname{Mcph1}^{\mathrm{NA}}$

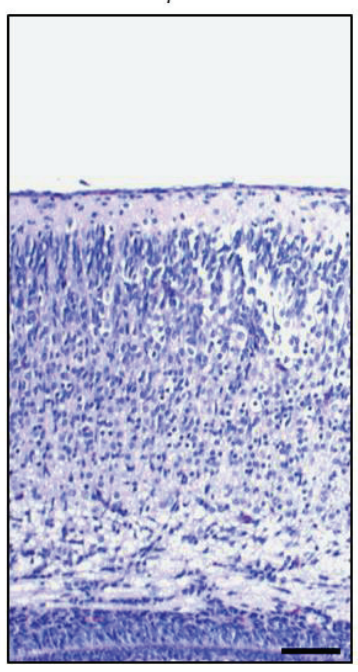

Figure 10: Reduced cerebral cortex size of $M c p h 1^{\Delta / \Delta}$ newborn (P0) mice.

(A) H\&E staining of brain coronal paraffin sections of PO mice. ctx = cerebral cortex; $h p=$ hippocampus; $\mathrm{mb}=$ midbrain; am = amygdala. Scale bars: $500 \mu \mathrm{m}$.

(B) Higher magnification of selected areas as indicated in A (rectangles). Scale bars: $50 \mu \mathrm{m}$. 
A

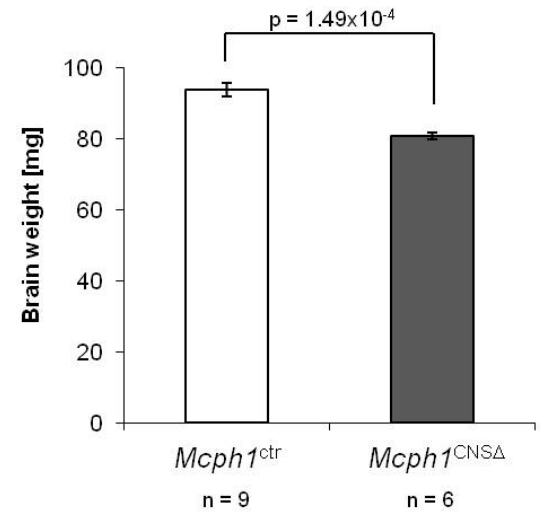

C

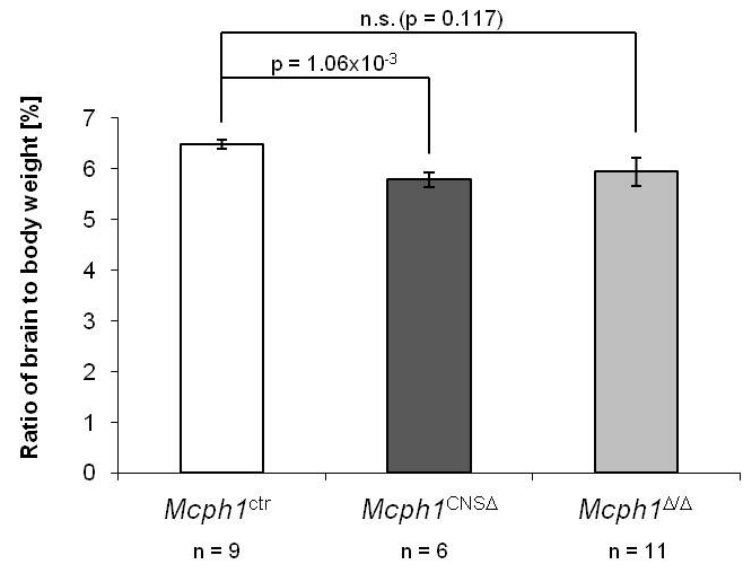

B

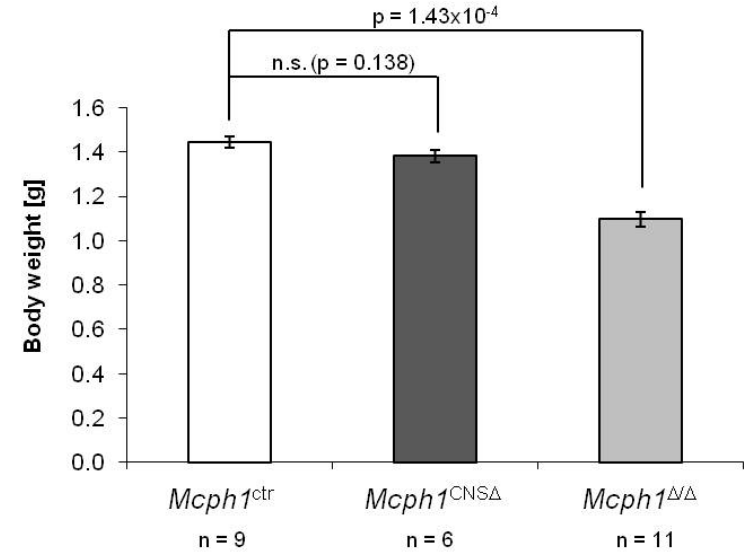

Figure 11: Comparison of brain and body weight of $M c p h 1^{\mathrm{CNS} \Delta}$ and $M c p h 1^{\Delta / \Delta}$ newborn (P0) mice.

(A) Brain weight of P0 mice.

(B) Body weight of P0 mice.

(C) Ratio of brain weight relative to body weight of P0 mice.

Means \pm standard error of means of $n=$ number of mice are shown. Student's t-test was performed for statistical analysis.

This enabled us to study the specific function of $\mathrm{MCPH} 1$ in neuronal progenitor cells during embryonic cortical development. $M c p h 1^{+/+}, M c p h 1^{+/ \Delta}, M c p h 1^{+/ T}$ and $M c p h 1^{+/ f l o x}$ mice were phenotypically indistinguishable, independently of the presence of the nestin-Cre transgene, and therefore grouped as control mice, designated as $M c p h 1^{\mathrm{ctr}}$. In addition, mice homozygous for the targeted $\left(M c p h 1^{\mathrm{T} / \mathrm{T}}\right)$ or floxed $\left(M c p h 1^{\text {flox/flox }}\right)$ alleles, but lacking the nestin-Cre transgene 
were used as control mice $\left(M c p h 1^{\mathrm{ctr}}\right)$. The $M c p h 1^{\mathrm{CNS} \Delta}$ mice showed a significant reduction of brain weight compared with $M c p h 1^{\text {ctr }}$ mice, as seen in $M c p h 1^{\Delta / \Delta}$ mice (Figure 11A). Of note, the body weight of the Mcph1 $1^{\mathrm{CNS} \Delta}$ mice was not affected (Figure 11B, C). The phenotype of the $M c p h 1^{\mathrm{CNS} \Delta}$ mice suggest, that $\mathrm{MCPH} 1$ has a specific function in neuronal progenitor cells or their progeny during embryonic cortical development.

\subsubsection{Mcph $1^{\Delta / \Delta}$ mice showed normal behaviour}

\subsubsection{Barnes maze test showed normal learning and memory ability of Mcph1 $/$ mice}

It has been reported that human $\mathrm{MCPH}$ patients show a mild to moderate mental retardation (Cox et al., 2006; Woods et al., 2005). We next wanted to address, whether deletion of Mcph1 in mice would resemble the human disease phenotype, resulting in a reduced capacity of learning and memory.

The Barnes maze test was chosen to assess the spatial learning and memory ability. The Barnes maze consists of a circular platform with 40 equally spaced holes surrounding the perimeter of the maze. The mice received reinforcement to escape from the open platform to a dark recessed chamber which is placed under one hole referred to as target hole. During the trial the number of errors (nose pokes in holes different from the target hole; Figure 12A) and the time until the mouse entered the target hole was recorded (Figure 12B) to evaluate the spatial learning ability. The animals received 4 trails per day during 4 consecutive days. In total 6 mice of the control group $M c p h 1^{\text {ctr }}$ and $6 M c p h 1^{\Delta / \Delta}$ mice were analyzed with the Barnes maze test. The number of errors and latency time as mean of all mice is shown in Figure 12A and Figure 12B, respectively. Despite the reduced brain size, $M c p h 1^{\Delta / \Delta}$ mice show no significant defects in spatial learning ability measured by the Barnes maze test. 
A

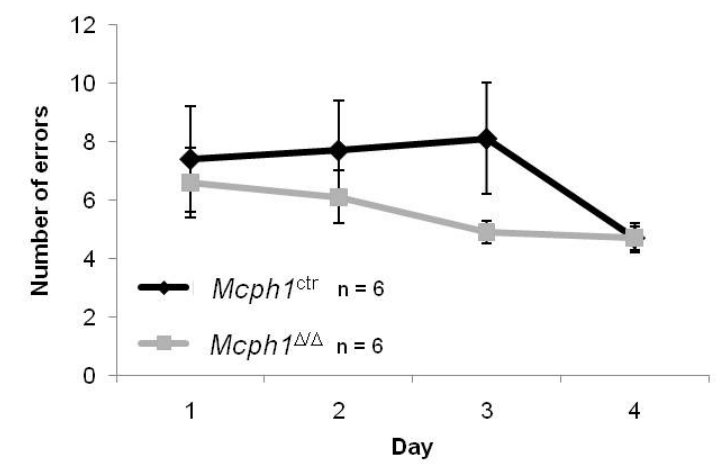

B

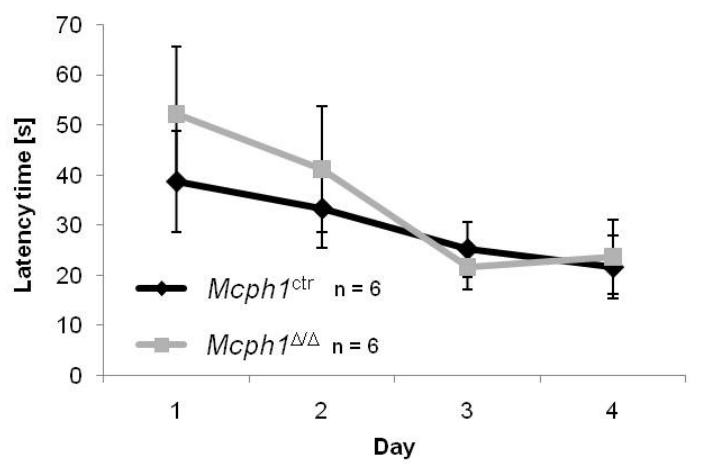

C

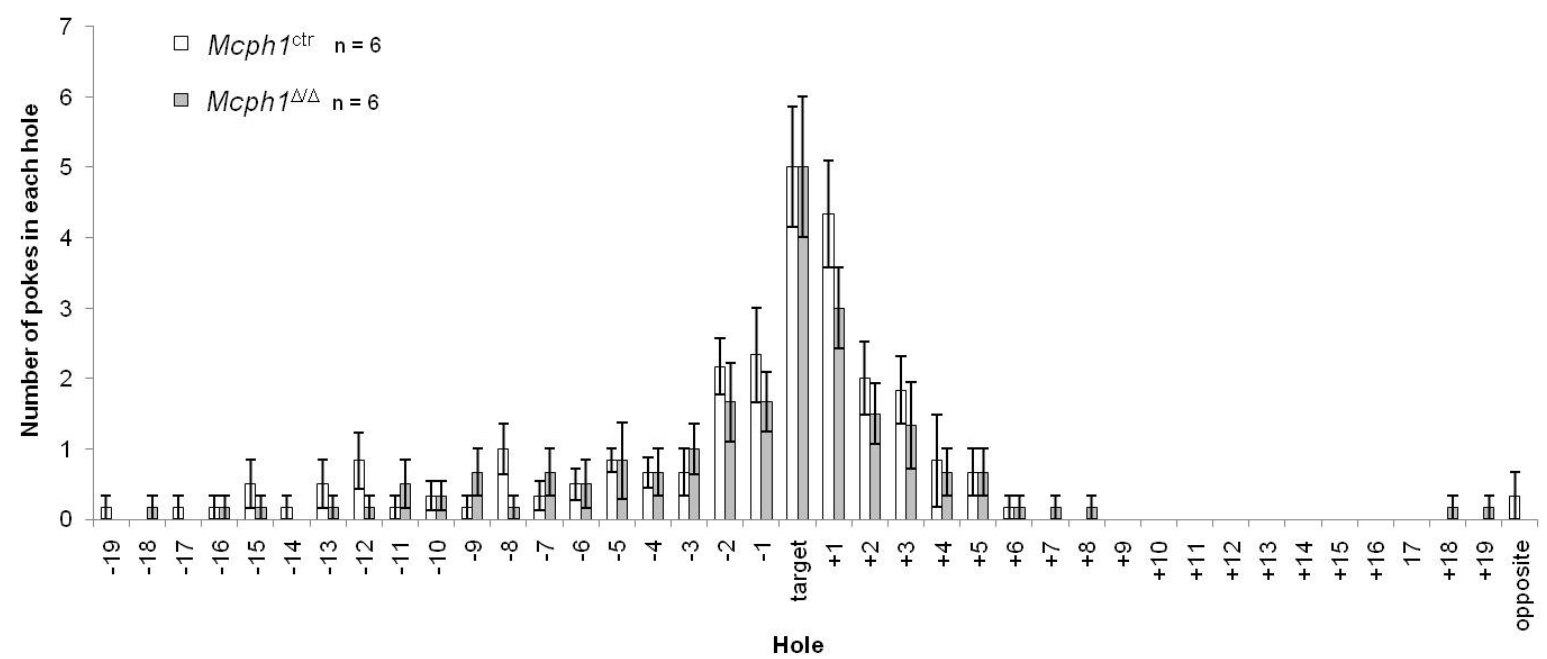

Figure 12: Barnes maze test shows normal spatial learning and memory ability of Mcph1 $^{\Delta / \Delta}$ mice.

(A, B) Barnes maze test to assess spatial learning ability was performed over four consecutive days.

(A) Number of errors was recorded as nose pokes into holes different from target hole.

(B) Latency time on the maze until reaching the target hole of the mice is shown.

(C) On day five the probe trail was used to assess spatial memory retention. Number of pokes into each hole was counted. Note the preference for the target hole.

Means \pm standard error of means of $n=$ number of mice are shown.

One day after the last trail the animals received the probe trail to assess the spatial short-term memory. The target box was removed and the mouse was allowed to explore the maze with reinforcement. During the probe trail, the number of nose pokes in each hole was recorded. 
The probe trial was performed to determine if the mouse remembers the location of the target hole. Similarly to the normal spatial learning ability, the $M c p h 1^{\Delta / \Delta}$ mice do not show a compromised spatial short-term memory in the Barnes maze test (Figure 12C).

\subsubsection{2 $\mathrm{Mcph}^{\Delta / \Delta}$ mice have normal motor coordination}

We next asked, whether $M c p h 1^{\Delta / \Delta}$ mice show behavioral deficits other than spatial learning and memory and measured the motor coordination using two different approaches. First, we tested these mice on the Rotarod. The mice were placed on a roller with accelerating speed and the persistence time on the roller was recorded. Figure 13A shows the time as mean of 5 mice per group and over the period of 5 consecutive days.

A

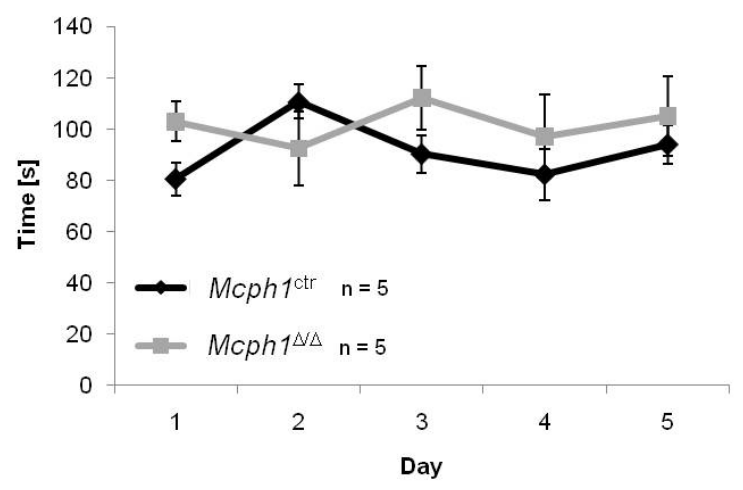

B

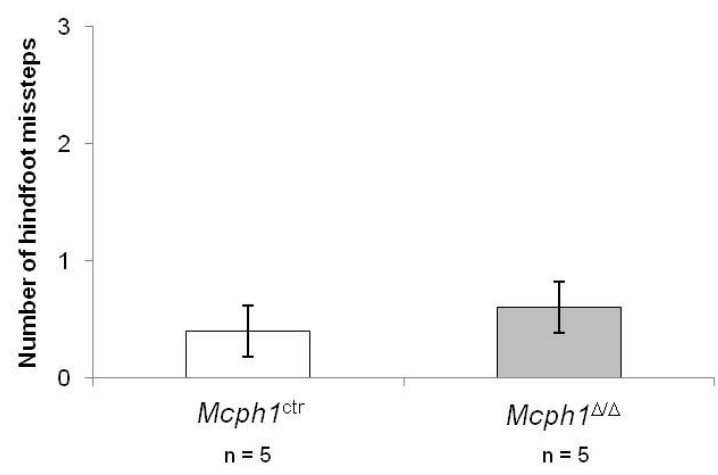

Figure 13: Rotarod and balance bar crossing test show normal motor coordination of Mcph1 $^{\Delta / \Delta}$ mice.

(A) Results from the Rotarod test. 5 mice per group were acclimated one before measurement at $5 \mathrm{rpm}$ for $5 \mathrm{~min}$. The next day the measurement was performed for 5 consecutive days. The measurement conditions were: start at $2 \mathrm{rpm}$; acceleration of $1 \mathrm{rpm} / 5 \mathrm{~s}$ to the maximum speed of $50 \mathrm{rpm}$. The persistence time of the mice on the Rotarod is shown as means \pm standard error of means of $\mathrm{n}=$ number of mice.

(B) Balance bar crossing test. 5 mice per group were allowed to cross the balance bar in 3 trails. Errors were recorded as hindfoot missteps, shown as means \pm standard error of means of $n=$ number of mice. 
Second, we determined the motor coordination of the mice using the balance bar crossing task. Mice were placed on a bar with the length of $1 \mathrm{~m}$ and allowed to cross the bar. Errors were counted as hindfoot missteps. In total, 5 mice per group were tested and each mouse performed 3 trials (Figure 13B). Mcph $1^{\Delta / \Delta}$ mice show no abnormalities in motor coordination measured by Rotarod as well as by the balance bar crossing test compared with $M c p h 1^{\text {ctr }}$ mice (Figure 13A, B).

\section{2 $\mathrm{Mcph}^{\Delta / \Delta}$ embryos have defects in neurogenesis}

\subsubsection{Reduced size of neuronal layer of $M c p h 1^{\Delta / \Delta}$ embryos}

To study the developmental basis of the reduction of cerebral cortex in postnatal Mcph $1^{\Delta / \Delta}$ mice, we analyzed the embryonic cortical development. During embryonic brain development, mainly the generation of neurons (neurogenesis) accounts for the massive increase in cell number of the CNS and therefore to the increase in brain size. The production of glial cells (gliogenesis) starts at very late embryonic stages and contributes to the increase in brain volume mainly postnatally (Kessaris et al., 2006; Mission et al., 1991). Since Mcph1 ${ }^{\Delta / \Delta}$ mice exhibit the microcephaly phenotype already at birth (Figures 9 and 10), we focused our experiments on the embryonic neurogenesis which persists in mice from E10 to E18 (Dehay and Kennedy, 2007).

We analyzed the embryonic cortex at the mid-phase of neurogenesis, at E15.5 and found a prominent reduction of the cortex size in the $M c p h 1^{\Delta / \Delta}$ embryos already at this stage (Figure 14A). A closer examination of the layering of the developing cortex revealed a dramatic reduction in radial thickness of the $\mathrm{CP}$, and of the neuronal migratory IZ (Figure 14B). However, the radial thickness of the proliferative areas, VZ and SVZ were less affected at E15.5 compared with littermate control embryos (Figure 14B).

To further confirm this observation, we stained the E15.5 embryonic brain sections with an antibody against the neuron specific marker $\beta$-tubulinllI. This staining revealed a dramatic 
reduction of the neuronal layers $\mathrm{CP}$ and IZ of the $M c p h 1^{\Delta / \Delta}$ embryos compared with $M c p h 1^{\text {ctr }}$ embryos, suggestion a significant reduction of neuronal cell types in $M c p h 1^{\Delta / \Delta}$ embryos at E15.5 (Figure 15).

A
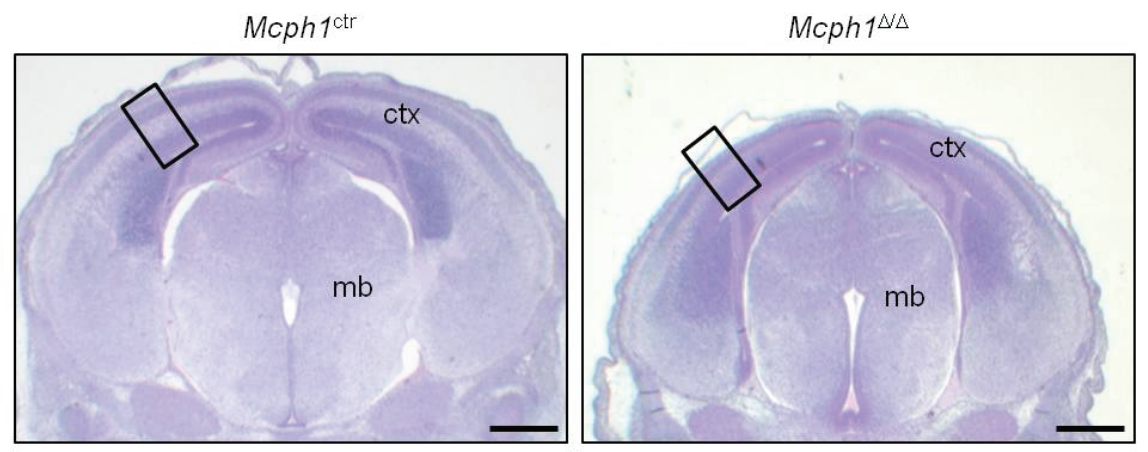

B

Mcph1 $1^{\mathrm{ctr}}$

$\operatorname{Mcph1} 1^{\mathrm{N} \Delta}$

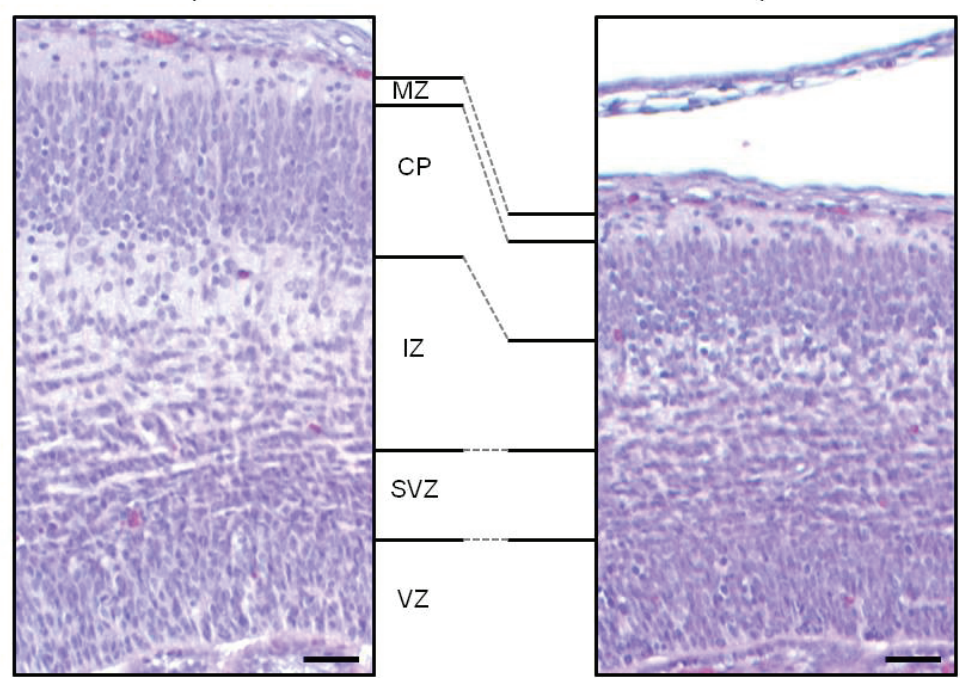

Figure 14: E15.5 $\mathrm{Mcph1}^{\Delta / \Delta}$ embryos show a reduced size of cortical plate and of intermediate zone, but not of proliferative zones.

(A) H\&E staining of brain coronal paraffin sections of E15.5 embryos. Scale bars: $500 \mu \mathrm{m}$.

(B) Higher magnification of selected areas as indicated in A (rectangles). Scale bars: $20 \mu \mathrm{m}$;

$\mathrm{ctx}=$ cerebral cortex; $\mathrm{mb}=$ midbrain; $\mathrm{VZ}=$ ventricular zone; SVZ = subventricular zone; $\mathrm{IZ}=$ intermediate zone; $\mathrm{CP}=$ cortical plate; $\mathrm{MZ}=$ marginal zone. 

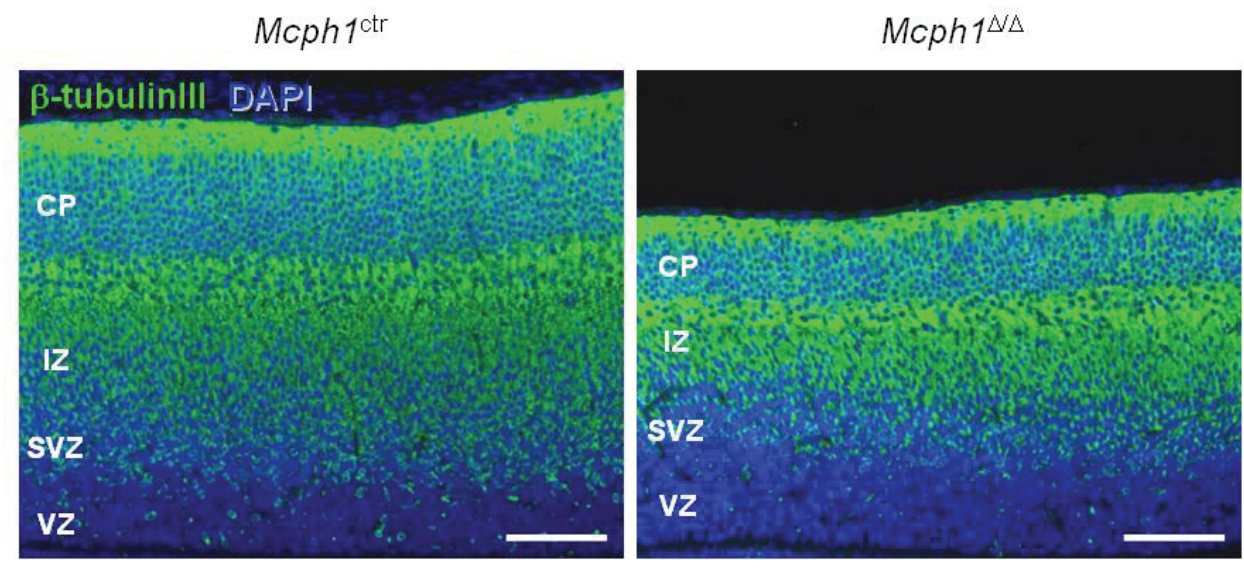

Figure 15: Reduced radial thickness of neuronal layers of E15.5 Mcph1 ${ }^{\Delta / \Delta}$ embryonic cortex.

Representative images of E15.5 sagittal brain sections stained with an antibody against the neuronal marker $\beta$-tubulinllI (green) and counterstained with DAPI (blue). Scale bars: $100 \mu \mathrm{m}$. VZ = ventricular zone; SVZ = subventricular zone; $I Z$ = intermediate zone; $C P=$ cortical plate.

\subsubsection{Normal proliferation rate in $M c p h 1^{\Delta / \Delta}$ developing cortex at mid-phase of neurogenesis (E15.5)}

We next examined the possible cause of the reduced size of the neuronal layer $\mathrm{CP}$ in the $M c p h 1^{\Delta / \Delta}$ embryos at E15.5. The normal thickness of the proliferative areas VZ, SVZ and the reduced size of the CP would suggest a slow proliferation rate of the neuronal progenitor cells, RGCs and/or IPCs in the Mcph $1^{\Delta / \Delta}$ embryos. A slow proliferation rate would lead to an insufficient generation of neurons and we therefore analyzed the proliferation rate in the dorsal telencephalon of E15.5 embryos.

To analyze the proliferation rate of the neuronal progenitor cells, we injected bromodeoxyuridine (BrdU), which can be incorporated into newly synthesized DNA during replication of S-phase cells, into pregnant mice at E15.5. One hour later the brains of the embryos were isolated and histological sections were prepared for immunohistological analysis. To determine the number and localization of replicated cells, an antibody against 
BrdU was used. Figure 16 shows sagittal brain sections after staining with a BrdU-antibody. BrdU-positive cells are located in the VZ and SVZ in both $M c p h 1^{\Delta / \Delta}$ and littermate control embryos, indicating that the deletion of Mcph1 does not affect the localization of S-phase neuronal progenitor cells (Figure 16A). We also quantified the BrdU-positive cells as a percentage of cells in VZ and SVZ and found no difference of $M c p h 1^{\Delta / \Delta}$ embryos compared with $M c p h 1^{\text {ctr }}$ embryos (Figure 16B). Hence, the reduction of the neuronal layer Mcph $1^{\Delta / \Delta}$ embryos at E15.5 is presumably not the result of a reduced proliferation rate of neuronal progenitor cells in the dorsal telencephalon.

\subsubsection{Reduced proliferation in the developing cortex of $M c p h 1^{\Delta / \Delta}$ embryos at the end of neurogenesis (E18.5)}

In addition to the determination of the proliferation at E15.5, the mid-phase of neurogenesis, we have analyzed the proliferation of neuronal progenitor cells in the telencephalon at the end of neurogenesis E18.5 by in vivo BrdU-labeling. Figure 16C shows examples of brain coronal sections of an E18.5 Mcph1 ${ }^{\Delta / \Delta}$ embryo and a littermate control, stained with a BrdU-antibody. Similarly to E15.5, in E18.5 embryos the main proportion of BrdU-positive cells are located in the VZ and the SVZ of the dorsal telencephalon. However, some BrdU-positive cells locate in the IZ or CP (Figure 16C). BrdU-positive cells which are not localized in the proliferative areas (VZ, SVZ) could be either newly born neurons which migrate to the CP or progenitor cells attributed to the glial cell lineage, which are not restricted to VZ/SVZ-localization (Mission et al., 1991). To analyze the proliferation of the neuronal progenitor cells at this embryonic stage, we quantified the total number of BrdU-positive cells in the dorsal telencephalon. We observed a significant reduction of BrdU-positive cells in $M c p h 1^{\Delta / \Delta}$ embryos at E18.5 compared with littermate control embryos (Figure 16D). 
A

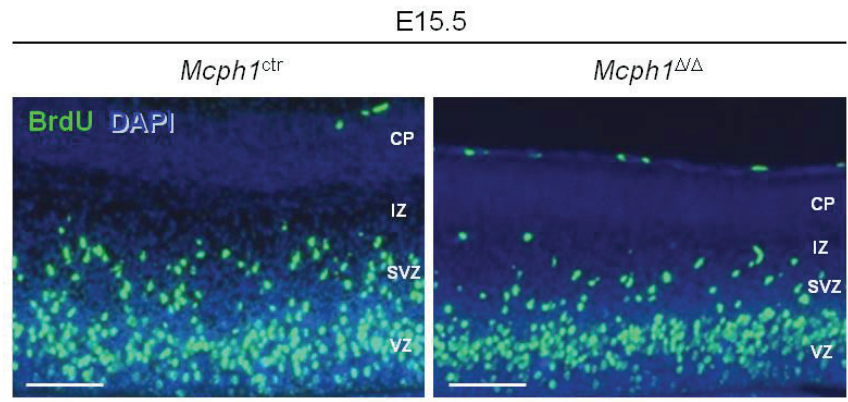

C

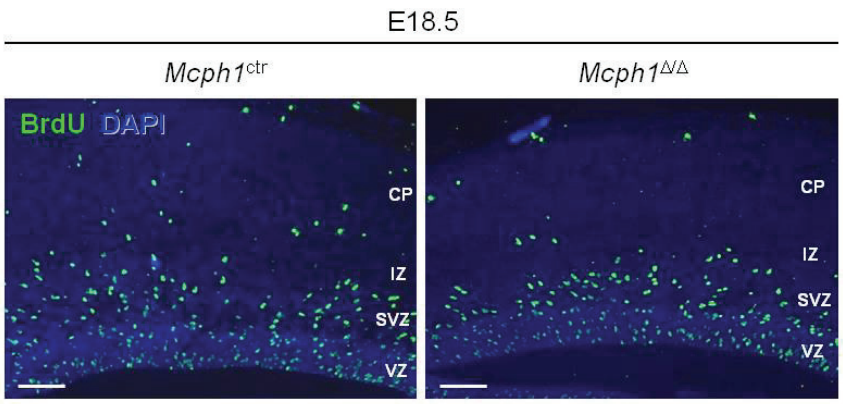

B

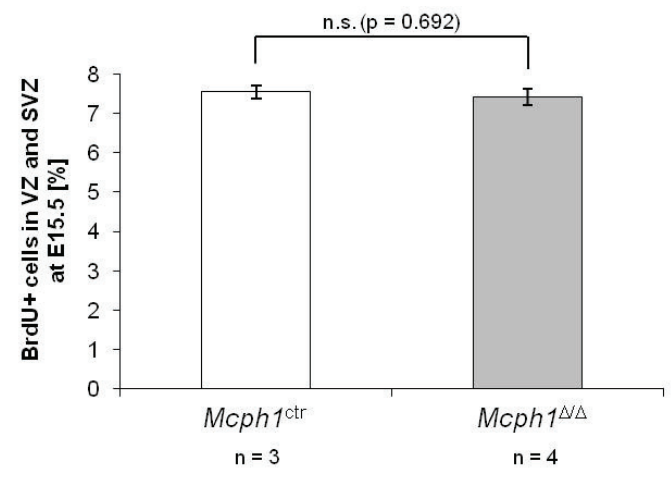

D

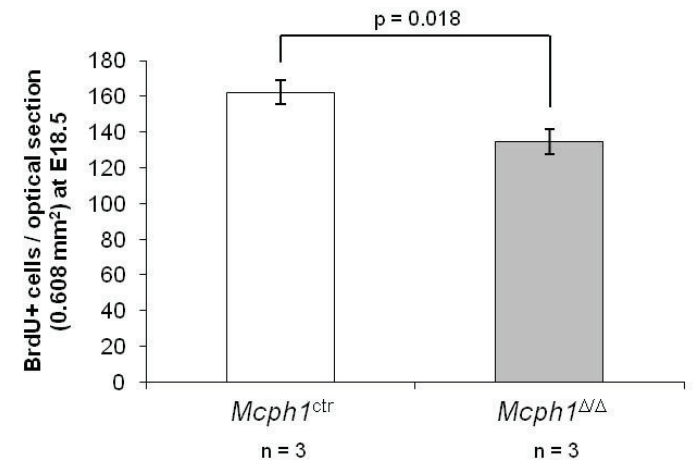

Figure 16: Reduced proliferation of $M c p h 1^{\Delta / \Delta}$ neuronal progenitors at end of neurogenesis (E18.5), but not at mid-phase of neurogenesis (E15.5).

(A) E15.5 embryos were pulse-labeled with BrdU in vivo and isolated $1 \mathrm{~h}$ later. Embryonic brain sagittal paraffin sections were stained with anti-BrdU-antibody (green) and counterstained with DAPI (blue) to determine proliferation rate of neuronal progenitor cells in the dorsal telencephalon E15.5. $\mathrm{VZ}=$ ventricular zone; SVZ = subventricular zone; $I Z=$ intermediate zone; $\mathrm{CP}=$ cortical plate. Scale bars: $100 \mu \mathrm{m}$.

(B) Quantification of BrdU-positive cells as percentage of cells in VZ and SVZ of E15.5 embryos. Means \pm standard error of means of $n=$ number of embryos are shown. Number of BrdU-positive cells: 1396 cells $M c p h 1^{\text {ctr. }} ; 1880$ cells $M c p h 1^{\Delta \Delta}$. Student's t-test was used for statistical analysis.

(C) BrdU pulse-labeling of E18.5 embryos in vivo and incubation for $1 \mathrm{~h}$. Brain coronal paraffin sections were stained with anti-BrdU-antibody (green) and counterstained with DAPI (blue) to determine proliferation rate of neuronal progenitor cells at E18.5. VZ = ventricular zone; SVZ = subventricular zone; $\mathrm{IZ}=$ intermediate zone; $\mathrm{CP}=$ cortical plate. Scale bars: $100 \mu \mathrm{m}$.

(D) Quantification of BrdU-postivie cells per optical section $\left(0.608 \mathrm{~mm}^{2}\right)$ in the telencephalon of E18.5 embryos. Means \pm standard error of means of $n=$ number of embryos are shown. Number of BrdUpositive cells: 1136 cells $M c p h 1^{\text {ctr }} ; 673$ cells $M c p h 1^{\Delta / \Delta}$. Student's t-test was used for statistical analysis. 


\subsubsection{Reduced number of cycling cells in $M c p h 1^{\Delta / \Delta}$ embryos at E17.5}

We next analyzed whether $M c p h 1^{\Delta / \Delta}$ embryos show a reduced number of neuronal progenitor cells at the end of neurogenesis which would explain the reduced number of BrdU-positive cells at E18.5. To quantify the number of neuronal progenitor cells, we used the cell cycle marker Ki67, which labels all cycling cells (RGCs and IPCs), but not postmitotic neurons.

A
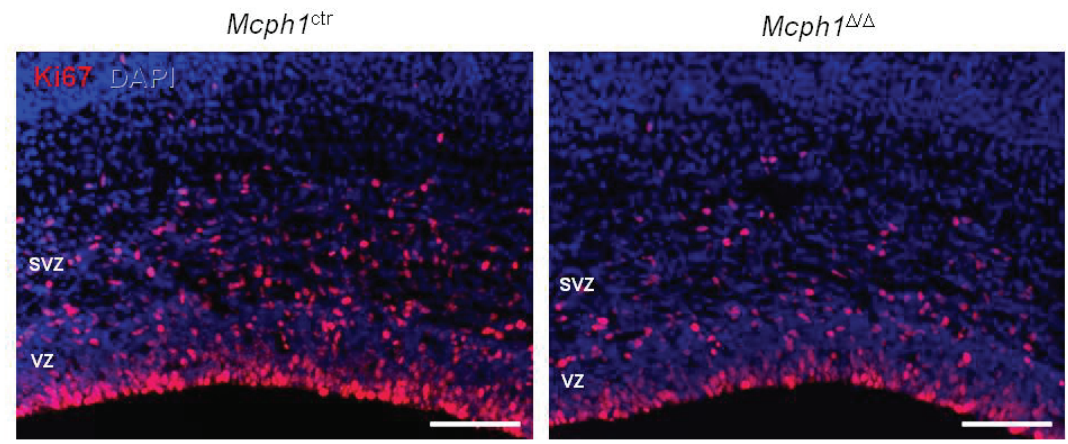

B

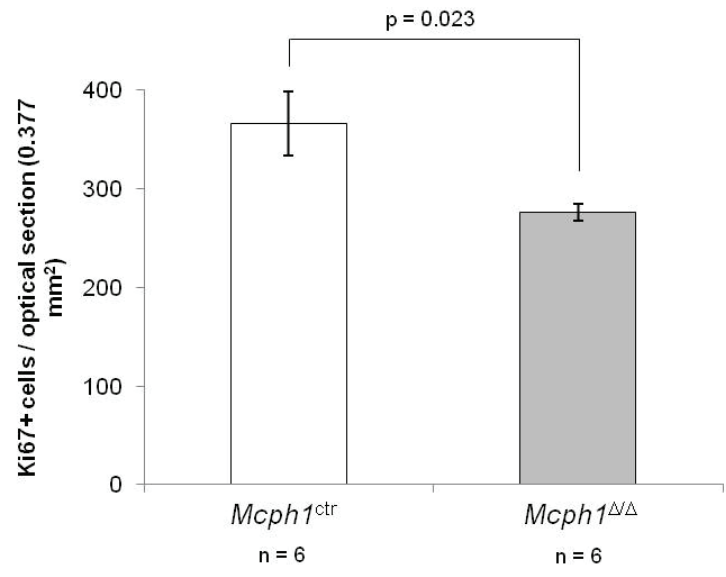

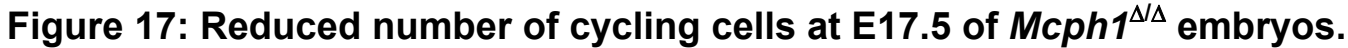

(A) Examples of E17.5 brain coronal cryosections stained with anti-Ki67-antibody (red) and counterstained with DAPI (blue) to analyze the number of cycling cells. VZ = ventricular zone; SVZ = subventricular zone. Scale bars: $50 \mu \mathrm{m}$.

(B) Quantification of Ki67-positive cells per optical area of $0.377 \mathrm{~mm}^{2}$. Means \pm standard error of means of $n=$ number of sections of 3 embryos are shown. Student's t-test was used for statistical analysis. 
In Figure 17A examples of embryonic brain sections at E17.5 after Ki67-antibody-staining are shown. The cycling cells (Ki67-positive) are located in the ventricular zone, presumably RGCs and in the subventricular zone, presumably IPCs. A quantification of Ki67-positive cells per

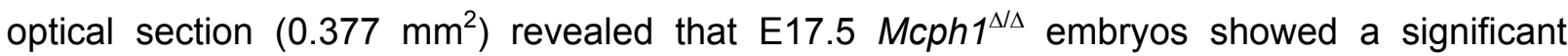
reduction of the Ki67-positive cell number compared to $M c p h 1^{\text {ctr }}$ embryos (Figure 17B).

\subsubsection{Increased apoptosis in the VZ and SVZ of the telencephalon in $M c p h 1^{\Delta \Delta}$ embryos}

Cell death by apoptosis could contribute to the loss of neuronal progenitor cells. To investigate the cause of the progenitor cell loss, we next analyzed cell death in the developing dorsal telencephalon. Apoptotic cells in tissue sections can be detected by the terminal deoxynucleotidyl transferase dUTP nick end labeling (TUNEL), which labels fragmented DNA, a hallmark of late stage apoptotic cells. The enzyme terminal deoxynucleotidyl transferase catalyzes an addition of dUTP, which can be biotin-linked, to 3'-hydroxyl ends of DNA singleor double-strand break. To visualize the breaks, fluorescence-labeled streptavidin can be used, which binds to the DNA-break-linked biotin.

We performed the TUNEL assay of brain sections of E13.5 and E15.5 embryos to study

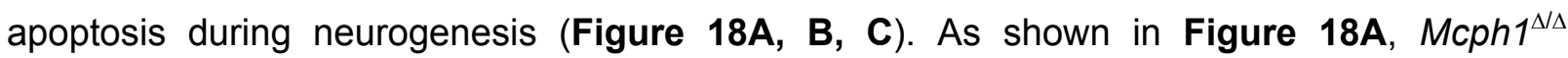
embryos at E15.5 show increased apoptosis in the VZ and SVZ of the dorsal telencephalon compared with Mcph $1^{\text {ctr }}$ embryos. Figure 18B shows a quantification of apoptotic cells per E15.5 brain section. Notably, the increase in apoptosis of $M c p h 1^{4 / \Delta}$ embryos seems to be restricted to the $V Z$ and $S V Z$, suggesting that apoptosis occurs specific in the neuronal progenitor cell population. A very similar result was obtained when we performed the TUNEL reaction on E13.5 brain sections. Figure 18C shows the quantification of TUNEL-positive cells, which reveals that apoptotic cells are significantly increased in the VZ and SVZ of the dorsal telencephalon in $M c p h 1^{\Delta / \Delta}$ embryos compared with control embryos, whereas other brain regions show no increase in apoptosis (Figure 18D). 
Since the apoptosis is restricted to the proliferative regions of the brain, the VZ and SVZ of the dorsal telencephalon, suggests that apoptosis might be triggered specifically in proliferating cells. To verify this hypothesis, we have analyzed the apoptosis in brains of adult mice, which are mainly postmitotic. We could not observe an increase of apoptosis in whole brain sections of $M c p h 1^{\Delta / \Delta}$ adult mice (Figure 18D). Hence, deletion of Mcph1 leads to apoptosis specific in proliferating brain regions during embryonic development, but not in postmitotic brain regions.

A
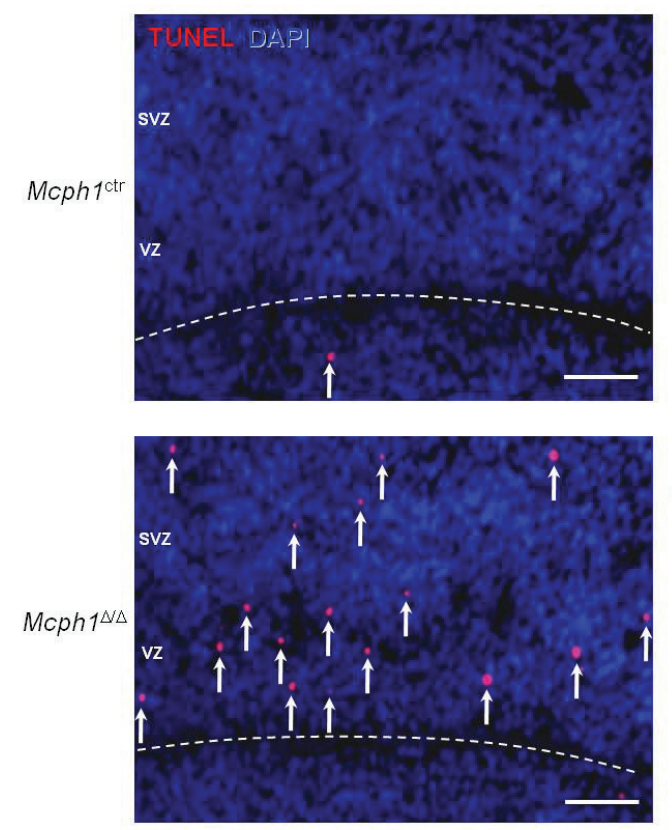

B

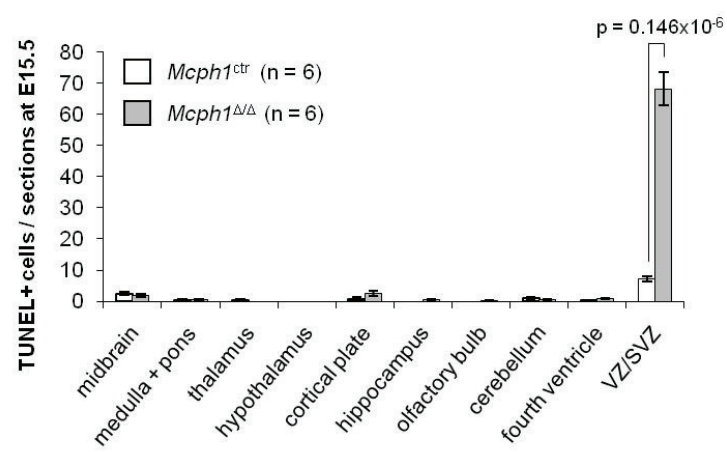

C

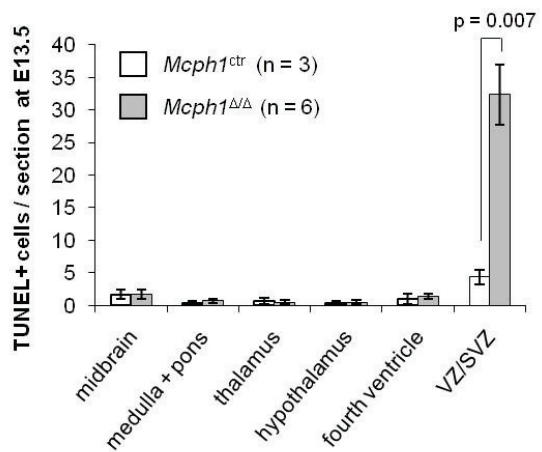

D

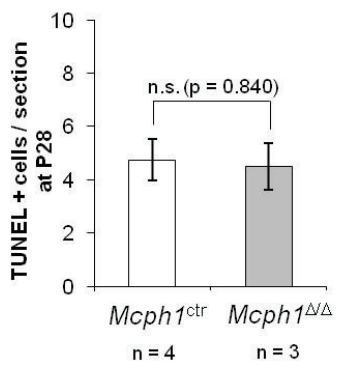

Figure 18: Increased apoptosis in $M c p h 1^{\Delta / \Delta}$ VZ and SVZ of embryonic brains at E13.5 and E15.5, but not in adult brains.

(A) TUNEL reaction was performed on E15.5 sagittal brain cryosections and counterstained with DAPI (blue). Apoptotic cells (TUNEL-positive, red staining, white arrows) are shown in the VZ and SVZ of the telencephalon. Dashed white lines indicate the lateral ventricle. Scale bars: $50 \mu \mathrm{m}$.

(B) Quantification of TUNEL-positive cells in E15.5 sagittal brain cryosections.

(C) Quantification of TUNEL-positive cells in E13.5 sagittal brain cryosections.

(D) Quantification of TUNEL-positive cells in whole sagittal brain cryosections of 4 weeks old mice (P28).

Means \pm standard error of means are shown of $n=$ number of embryos (in $B, C$ ) or $n=$ number of mice (in D). Student's t-test was performed for statistical analysis; n.s. = not significant. VZ = ventricular zone; SVZ = subventricular zone. 


\subsubsection{No chromosomal aberrations of $M c p h 1^{\Delta / \Delta}$ neuronal progenitor cells}

Apoptosis could be caused by the accumulation of unrepaired DNA breaks in $M c p h 1^{\Delta / \Delta}$ neuronal progenitor cells. To test whether there is an accumulation of cells with unrepaired DNA breaks, we stained embryonic brain sections with an antibody against the phosphorylated histone variant $\mathrm{H} 2 \mathrm{AX}(\gamma-\mathrm{H} 2 \mathrm{AX})$ which marks sites of DNA double strand breaks (Rogakou et al., 1998). Figure 19A shows examples of cells in the $V Z$ of E15.5 embryos with $\gamma$-H2AX foci. A quantification of cells in the VZ and SVZ containing $\gamma-\mathrm{H} 2 \mathrm{AX}$ foci showed a normal number of $M c p h 1^{\Delta / \Delta}$ cells compared with control cells (Figure 19B).

A

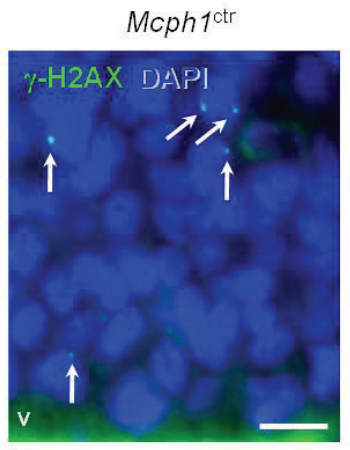

B

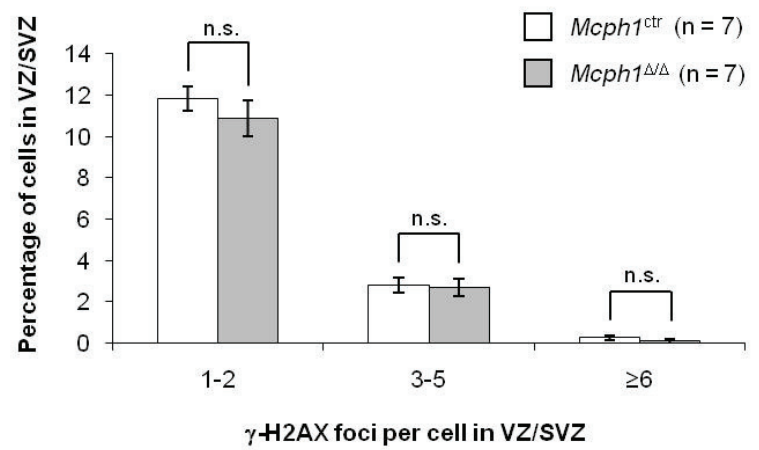

Figure 19: Normal number of $\gamma-\mathrm{H} 2 A X$ foci in $M c p h 1^{\Delta / \Delta}$ VZ and SVZ in embryonic brains at E15.5.

(A) Examples of E15.5 brain sagittal cryosections stained with anti- $\gamma-\mathrm{H} 2 \mathrm{AX}$-antibody (green) and counterstained with DAPI (blue) to analyze the number of $\gamma-\mathrm{H} 2 \mathrm{AX}$ foci (marker for DNA double strand breaks). White arrows indicated $\gamma-\mathrm{H} 2 \mathrm{AX}$ foci. Cells in the $\mathrm{VZ}$ are shown. $\mathrm{V}=$ lateral ventricle. Scale bars: $10 \mu \mathrm{m}$.

(B) Quantification of $\gamma-\mathrm{H} 2 \mathrm{AX}$ foci per cell in the VZ of E15.5 embryos. Mean \pm standard error of means are shown of $\mathrm{n}=$ number of embryos. Number of cells analyzed: 1596 cells Mcph1 $1^{\text {ctr; }} 1961$ cells $M c p h 1^{\Delta / \Delta}$. Student's t-test was performed for statistical analysis. 
Next, we analyzed chromosomal aberrations of neuronal progenitor cells in vitro. We isolated neuronal progenitor cells from E14.5 embryos and after 7 days in vitro, we analyzed their chromosomes after metaphase arrest. We found a comparable number of chromosomal aberrations (breaks, gaps and fusions) between $M c p h 1^{\Delta / \Delta}$ and $M c p h 1^{\text {ctr }}$ neuronal progenitor cells (Table 3). Thus, the increased apoptosis in the VZ and SVZ of Mcph $1^{\Delta / \Delta}$ embryos seems not to be caused by genomic instability.

Table 3: No chromosomal aberrations of $M c p h 1^{\Delta / \Delta}$ neuronal progenitor cells.

S.e.m. = standard error of mean.

\begin{tabular}{ccccccc}
\hline Cell population & $\begin{array}{c}\text { Metaphases } \\
\text { analyzed }\end{array}$ & $\begin{array}{c}\text { Number of } \\
\text { chromosomesper } \\
\text { metaphase }\end{array}$ & $\begin{array}{c}\text { Chromosome } \\
\text { breaks } \\
\text { [Total number] }\end{array}$ & $\begin{array}{c}\text { Chromosome } \\
\text { gaps }\end{array}$ & $\begin{array}{c}\text { Chromosome } \\
\text { fusions }\end{array}$ & $\begin{array}{c}\text { Aberrations per } \\
\text { metaphase }\end{array}$ \\
\hline [Total number] & [Total number] & [Total number] & [\%] \\
Mcph $1 \Delta \Delta$ & 31 & $39.5 \pm 1.4$ & 1 & 1 & 0 & 6.5 \\
\hline
\end{tabular}

\section{3 $\mathrm{Mcph}^{\Delta / \Delta}$ neuronal progenitor cells show impaired self- renewal capacity in vitro}

\subsubsection{Normal proliferation of $M c p h 1^{\Delta / \Delta}$ E14.5 neuronal progenitor cells and P0 CNS-progenitor cells in vitro}

The in vivo BrdU-labeling study suggested no alteration of the proliferation capacity. We next analyzed the proliferation capacity of neuronal progenitor cells in an in vitro assay that allows the measurement of proliferation over several days. To this end, we isolated neuronal progenitor cells from E14.5 embryos and progenitors from brains of newborn (P0) mice and 
performed neurosphere formation assay which allows the measurement of proliferation in vitro. After isolation, these cells were cultured under defined conditions which prevent differentiation. The neuronal progenitor cells in vitro form non-adherent spherical cell aggregates, referred to as neurospheres. In Figure 20A examples of primary neurospheres (passage number 0, p0) 7 after isolation (7 days in vitro, DIV7) from E14.5 embryos of the indicated genotypes are shown. First, to determine the proliferation rate of neuronal progenitors at the mid-phase of neurogenesis, the numbers of primary neurospheres of E14.5 embryos at DIV7 were counted. Neuronal progenitor cells isolated from $M c p h 1^{\Delta / \Delta}$ E14.5 embryos form the same number of primary neurospheres as compared with $M c p h 1^{\text {ctr }}$ neuronal progenitor cells (Figure 20B). In addition to the neurosphere number, the size of the neurospheres was determined, by generating a single cell suspension of at least 100 neurospheres per embryo at DIV7 followed by cell counting. Consistent with the data on in vivo proliferation, the Mcph1 $1^{\Delta / \Delta}$ E14.5 primary neurospheres showed no defect in proliferation in vitro by measuring the size of neurospheres (cells per neurosphere) compared with $M c p h 1^{\text {ctr }}$ primary neurospheres (Figure 20C).

Second, the proliferation rate of CNS progenitor cells after the end of neurogenesis was measured. CNS progenitor cells were isolated from brains of newborn mice (P0) and allowed to form primary neurospheres in vitro. Figure 20D shows that $M c p h 1^{\Delta / \Delta} \mathrm{PO} C N S$-progenitor cells show a reduced number of formed neurospheres compared with Mcph $1^{\text {ctr }}$ neurospheres, however the difference was not significant ( $p=0.165$ by Student's t-test). Similarly, the size of the $M c p h 1^{\Delta / \Delta}$ P0 primary neurospheres was reduced compared with control neurospheres as measured by cells per neurosphere (Figure 20E), however no significant difference was observed ( $p=0.084$ by Student's t-test).

These results show that $M c p h 1^{\Delta / \Delta}$ neuronal progenitor cells have a normal proliferation capacity at mid-phase and at the end of neurogenesis. The reduced number and size of $M c p h 1^{\Delta / \Delta}$ neurospheres isolated from newborn mice might indicate a reduced number of progenitor cells at the time of isolation, the difference however was not significant. 
A

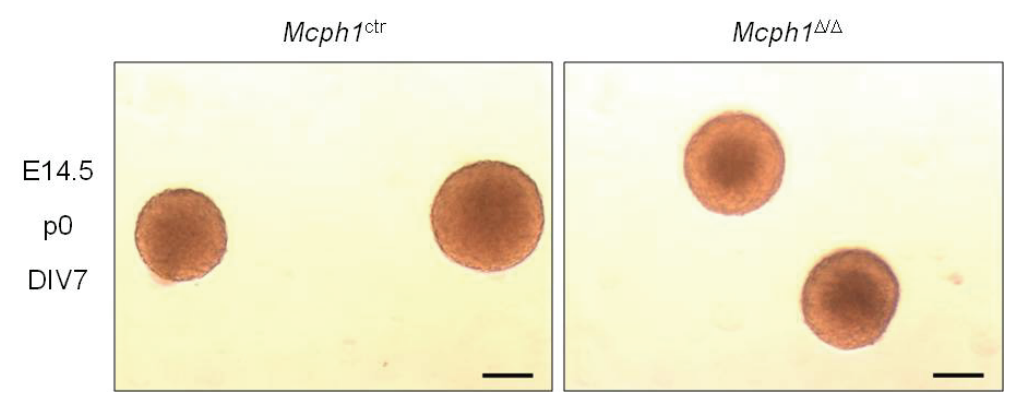

B

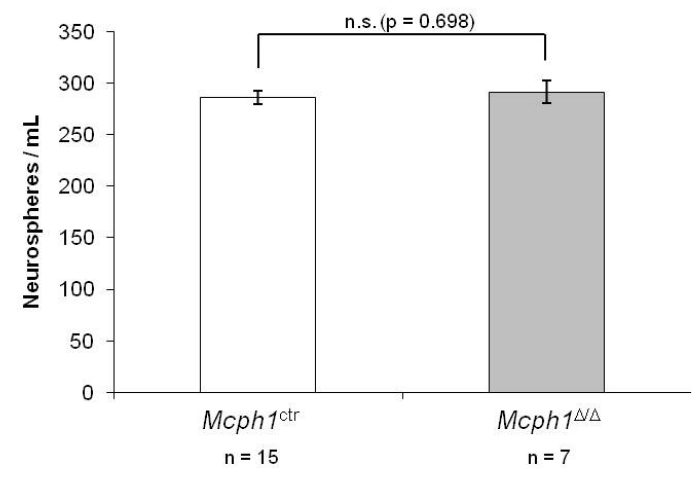

D

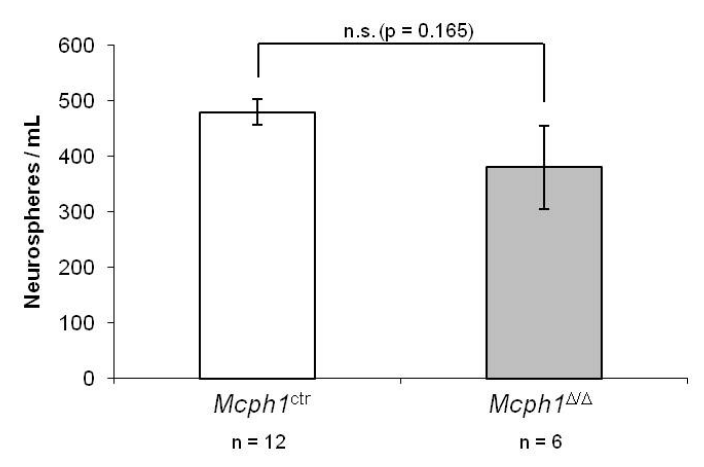

C

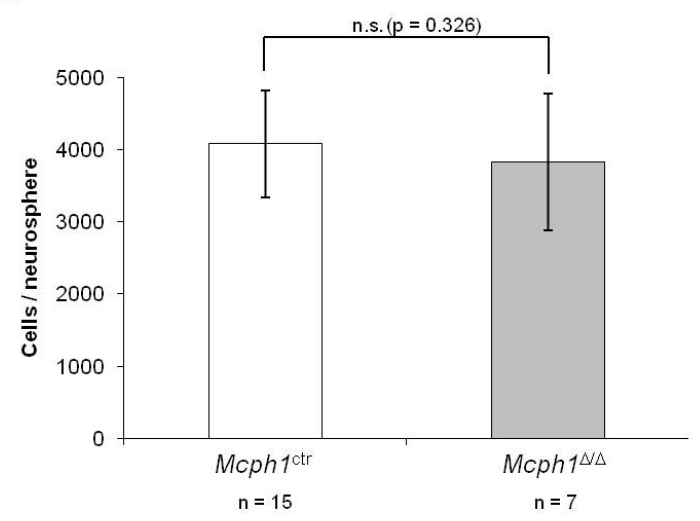

E

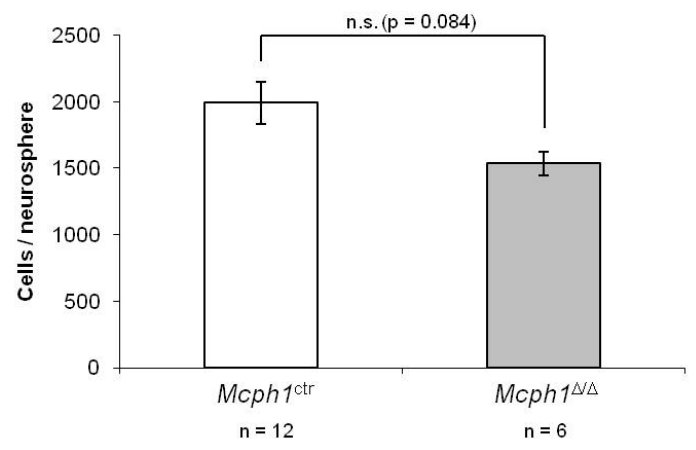

Figure 20: Normal proliferation of $\mathrm{Mcph}^{\Delta / \Delta} \mathrm{E} 14.5$ and P0 neurospheres in vitro.

(A) Primary neurospheres at passage number $0(\mathrm{p} 0), 7$ days in vitro (DIV7) isolated from E14.5 embryos of the indicated genotypes are shown. Scale bars: $200 \mu \mathrm{m}$.

(B) Numbers of E14.5 primary neurospheres (p0) per $\mathrm{mL}$ at DIV7 are shown.

(C) Sizes of E14.5 primary neurospheres (p0) as measured by cells per neurosphere at DIV7 are shown.

(D) Numbers of P0 primary neurospheres $(\mathrm{p} 0)$ per $\mathrm{mL}$ at DIV7 are shown.

(E) Sizes of P0 primary neurospheres $(\mathrm{p} 0)$ as measured by cells per neurospheres at DIV7 are shown. Means \pm standard error of means are shown of $n=$ number of embryos in $B$ and $C$ or $n=$ number of mice in D. Student's t-test was performed for statistical analysis; n.s. $=$ not significant. 


\subsection{2 $\mathrm{Mcph}^{\Delta / \Delta}$ neuronal progenitor cells show reduced self-renewal capacity in vitro}

The property of stem or progenitor cells is their ability to self-renew and to differentiate. The observed reduced proliferation of neuronal progenitor cells at E18.5 of $M c p h 1^{\Delta / \Delta}$ embryos could be loss of cells as a consequence of decreased self-renewal capacity in addition to the observed apoptosis. We therefore analyzed the self-renewal capacity of the neuronal progenitor cells in vitro by determining the capacity of E14.5 primary neurospheres to form secondary neurospheres after dissociating and replating.

The numbers of secondary neurospheres and in addition the size of the neurospheres were determined at DIV14. Mcph1 $1^{\Delta / \Delta}$ secondary neurospheres are slightly reduced in number of neurospheres (Figure 21B) as well as are slightly reduced in size as measured by cells per

neurosphere (Figure 21C) compared with secondary neurospheres isolated from control embryos. No significant difference was observed when we applied a statistical test (Student's t-test, see Figure 21B, C for p-values).

However, when we analyzed the ability of the secondary neurospheres $(p 1)$ to form tertiary neurospheres (p2), we found significant differences. Secondary neurospheres at DIV14 were dissociated, $1 \times 10^{4}$ cells were seeded again and allowed to form tertiary neurospheres for additional 7 days. We noted a dramatic reduction in the size of the $M c p h 1^{\Delta / \Delta}$ tertiary neurospheres (examples are shown in Figure 22A). When we measured the cells per neurospheres to determine the neurosphere size, we found a significant size reduction of the $M c p h 1^{\Delta / \Delta}$ tertiary neurospheres (Figure 22C). Similarly, Mcph $1^{1 / \Delta}$ neuronal progenitor cells show a significantly reduced number of tertiary neurospheres per $\mathrm{mL}$ (Figure 22B). The results show that Mcph1-deficient neuronal progenitor cells have a reduced self-renewal capacity compared with control cells, whereas the proliferation capacity of this cell type is not affected. 
A

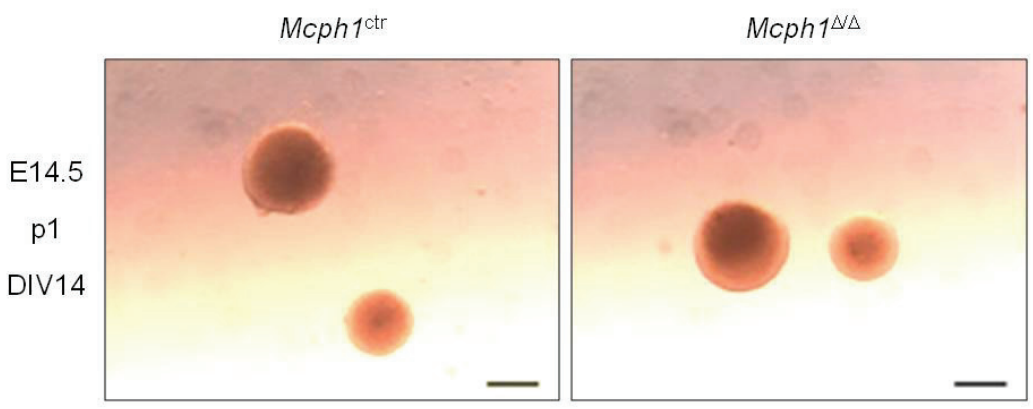

B

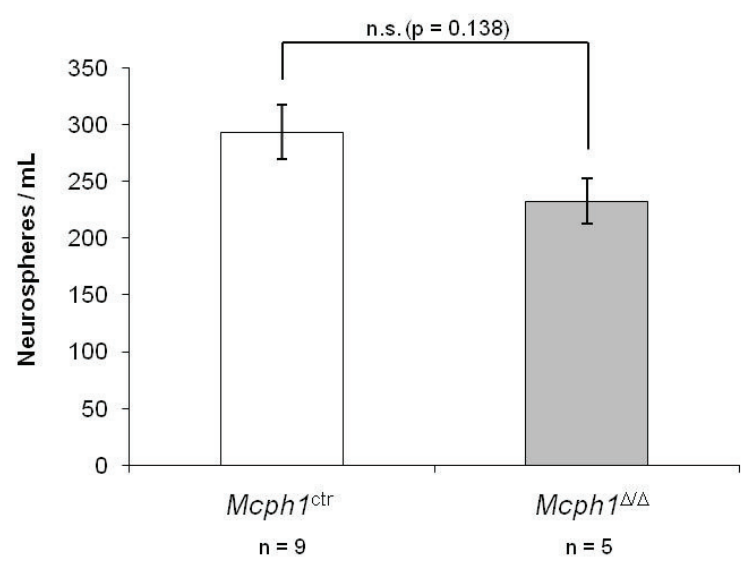

C

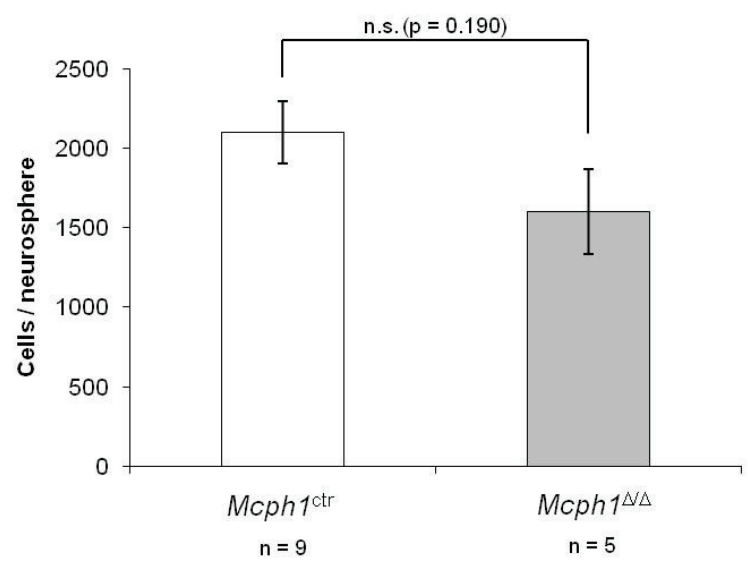

Figure 21: $M c p h 1^{\Delta / \Delta}$ E14.5 neuronal progenitor cells are able to self-renew in vitro.

(A) Examples of secondary neurospheres (passage number 1, p1) at DIV14. $1 \times 10^{4}$ cells were seeded after dissociation of E14.5 primary neurospheres at DIV7. Scale bars: $200 \mu \mathrm{m}$.

(B) Numbers of E14.5 secondary neurospheres (p1) per $\mathrm{mL}$ at DIV14 are shown.

(C) Sizes (cells/neurosphere) of E14.5 secondary neurospheres (p1) at DIV14 are shown.

Means \pm standard error of means are shown of $n=$ number of embryos. Student's t-test was performed for statistical analysis; n.s. = not significant. 
A

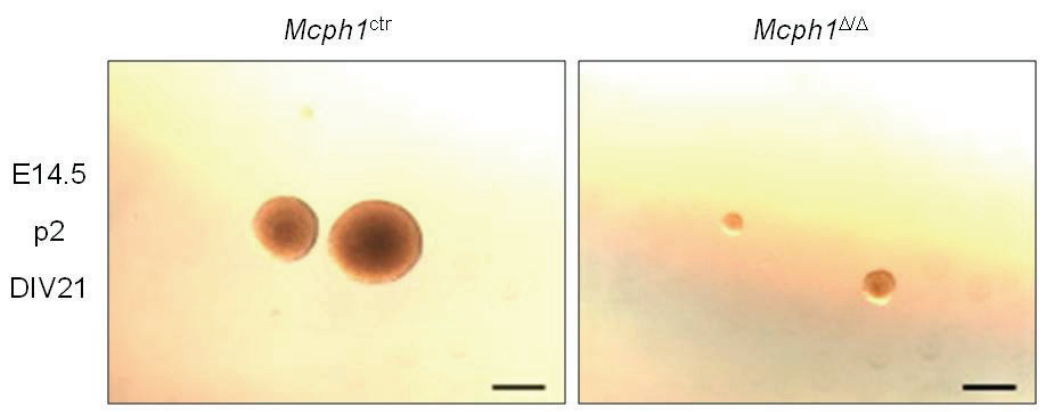

B

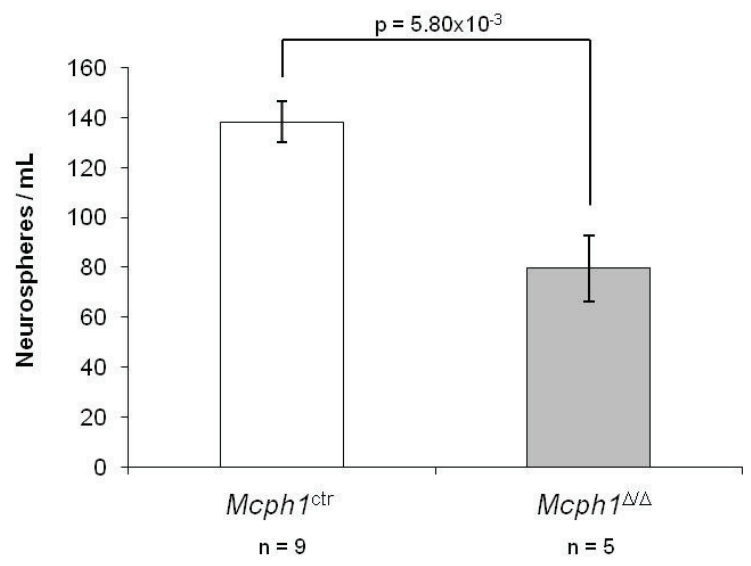

C

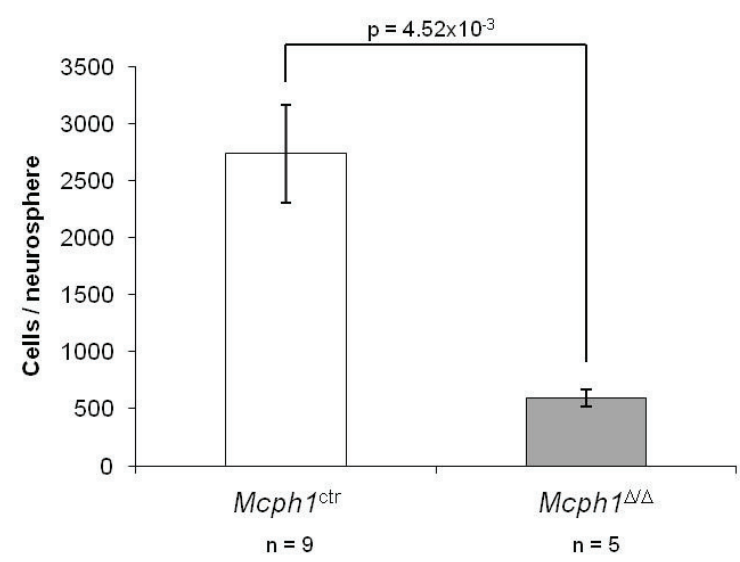

Figure 22: Reduced self-renewal capacity of $\operatorname{Mcph}^{\Delta / \Delta}$ E14.5 neuronal progenitor cells in vitro.

(A) Examples of tertiary neurospheres (passage number 2, p2) at DIV21. Secondary neurospheres were dissociated at DIV14 and $1 \times 10^{4}$ cells were seeded to form tertiary neurospheres. Scale bars: $200 \mu \mathrm{m}$.

(B) Numbers of E14.5 tertiary neurospheres (p2) per $\mathrm{mL}$ at DIV21 are shown.

(C) Sizes of E14.5 tertiary neurospheres (p2) as measured by cells per neurosphere at DIV21 are shown.

Means \pm standard error of means are shown of $n=$ number of embryos. Student's t-test was performed for statistical analysis; n.s. = not significant. 


\subsection{Mcph1 ${ }^{\Delta / \Delta}$ embryos show increased asymmetric progenitor cell division in the dorsal telencephalon}

\subsection{1 $\mathrm{Mcph}^{\Delta / \Delta}$ embryos show an increased cell cycle exit in the dorsal telencephalon}

In addition to the ability to self-renew, stem and progenitors cells are defined by the ability to differentiate. The previous results showed that $M c p h 1^{\Delta / \Delta}$ neuronal progenitor cells have a normal proliferation capacity, whereas their self-renewal capacity is compromised. We next asked, whether Mcph1-deficient neuronal progenitor cells show an altered differentiation capacity. Since the $M c p h 1^{\Delta / \Delta}$ mice exhibit the microcephaly phenotype at birth, it suggests a compromised embryonic neurogenesis. A possible defective postnatal gliogenesis might contribute to the primary microcephaly observed in $M c p h 1^{1 / \Delta}$ newborn mice to a less extend. We therefore decided to determine the differentiation ability in an in vivo assay: the cell cycle exit index, which allows the measurement of the differentiation rate of RGCs and IPCs specific into neurons in the embryonic cortex.

Pregnant mice at E14.5 were injected with a single dose of BrdU to label proliferating cells in $\mathrm{S}$-phase during the time of injection. The mother was sacrificed $24 \mathrm{~h}$ after the injection and brains of the E15.5 embryos were isolated and prepared for immunohistochemical analysis. Histological embryonic brain sections were stained with antibodies against BrdU and the cell cycle marker Ki76. The BrdU-antibody labels cells which have been in S-phase at the time of BrdU-injection. The Ki67-antibody is used as a cell cycle marker, which stains proliferating cells in all cell cycle phase. Cells in G0-phase are not stained by this antibody. 
A

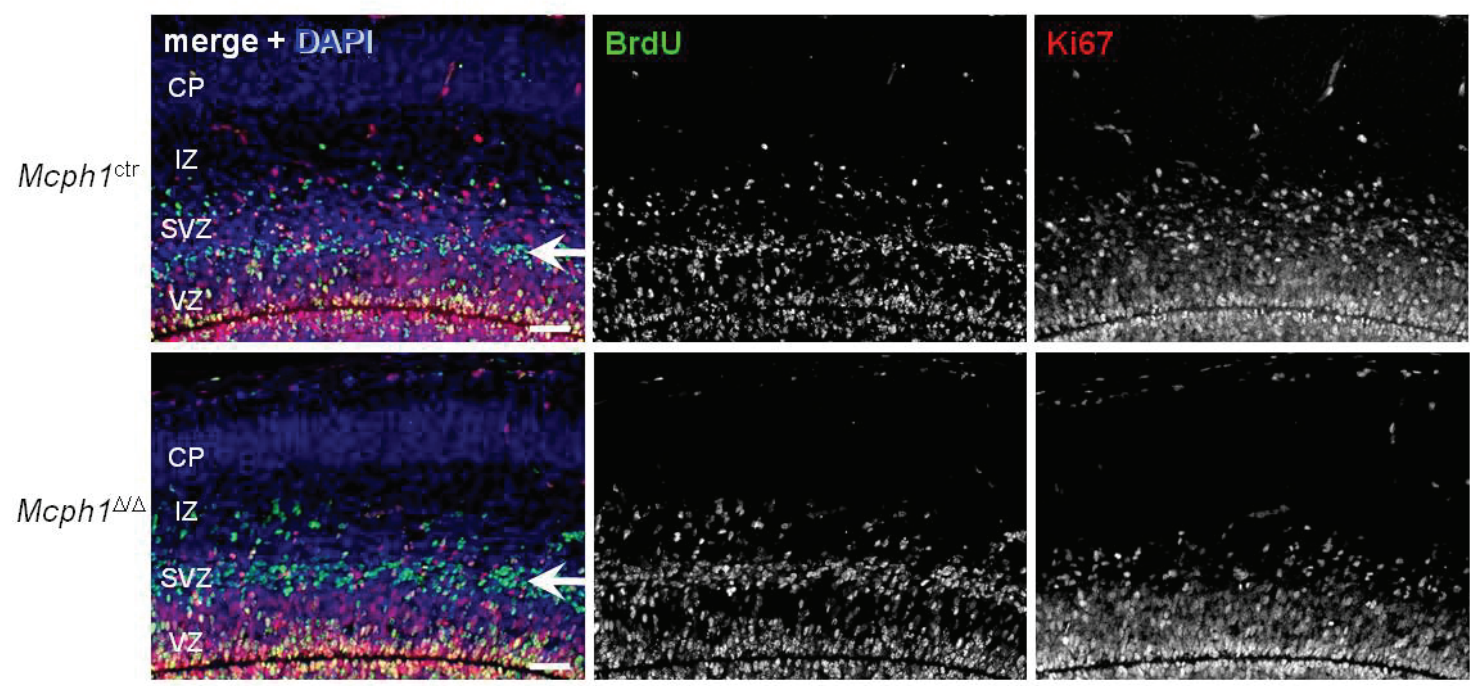

B
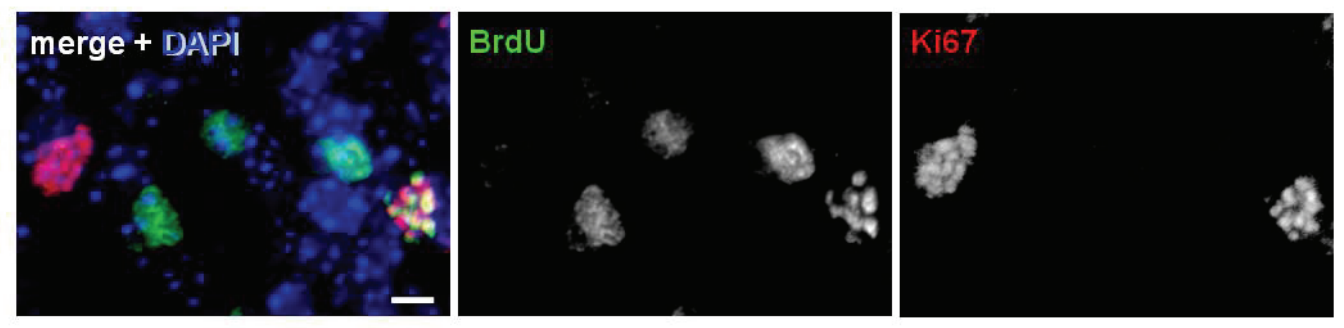

C

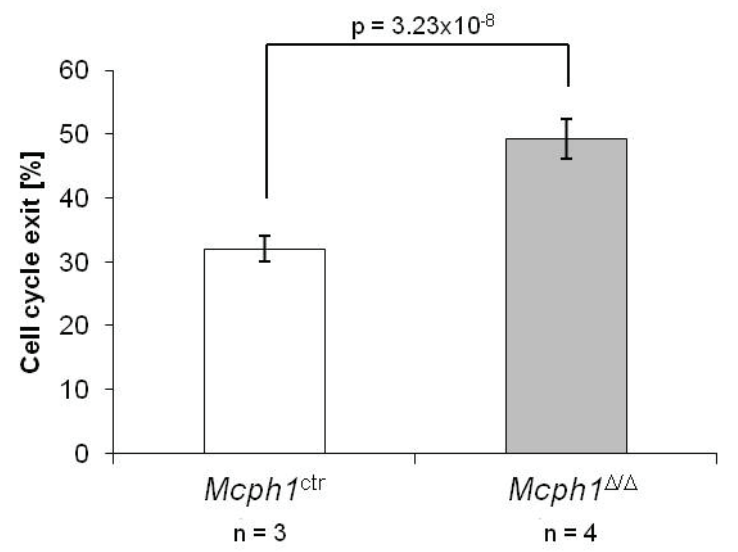

Figure 23: Increased cell cycle exit of $M c p h 1^{\Delta / \Delta}$ embryos in the dorsal telencephalon.

(A) E14.5 embryos were pulse-labeled with BrdU in vivo and isolated $24 \mathrm{~h}$ later. E15.5 coronal brain sections after anti-BrdU- (green) and anti-Ki67- (red) antibody-staining and staining with DAPI (blue) are shown. White arrows indicate layers with high number of cells exited the cell cycle. $V Z=$ ventricular zone; SVZ = subventricular zone; $I Z$ = intermediate zone; $C P=$ cortical plate. Scale bars: $50 \mu \mathrm{m}$.

(B) High magnification of labeled cells in the telencephalon: i) single Ki67-positive cell (red) ii) single BrdU-positive cells (green), iii) double BrdU- and Ki67-positive cells (yellow). Scale bar: $5 \mu \mathrm{m}$.

(C). Cell cycle exit index of neuronal progenitor cells (single BrdU-positive cells as percentage of all BrdU-positive cells at E15.5 embryos after BrdU-pulse labeling at E14.5). Means \pm standard error of means of $\mathrm{n}=$ number of embryos are shown. Number of scored BrdU-positive cells: 4553 cells Mcph $1^{\text {ctr }}$; 7730 cells $M c p h 1^{\Delta / \Delta}$. Student's t-test was used for statistical analysis. 
The double staining procedure with the two antibodies against BrdU and Ki67 revealed three different types of labeled cells (Figure 23B): i) single BrdU-positive cells are those cells which have been in S-phase at the time of BrdU-injection, but exited the cell cycle to become potentially postmitotic neurons (Figure 23B, green cells); ii) BrdU-Ki67-double positive cells have been in S-phase at time of injection and are still in the cell cycle potentially as neuronal progenitor cells in the dorsal telencephalon (Figure 23B, yellow cell); iii) single Ki67-positive cells are cycling cells (progenitor cells), which have not been in S-phase at the time of injection (Figure 23B, red cells). The cell cycle exit index was calculated as a number of cells that exited the cell cycle (BrdU-positive and Ki67-negative cells) as a percentage of the total BrdUpositive cells (Ki67-positive and Ki67-negative cells).

Figure 23A shows cortices of E15.5 brain sections of control and Mcph $1^{\Delta / \Delta}$ embryos, which were stained with antibodies against BrdU (green) and Ki67 (red) after BrdU-pulse labeling at E14.5, to determine the cell cycle exit index. In $M c p h 1^{\Delta / \Delta}$ embryos, we observed an increased number of single BrdU-positive cells in the SVZ and IZ, when compared with littermate control embryos (Figure 23A). To quantify the neuron production rate, we calculated the cell cycle exit index from E14.5 to E15.5. $M c p h 1^{\Delta / \Delta}$ embryos show a much higher cell cycle exit index compared with littermate control embryos (Figure 23C). This result shows that Mcph1 deletion promotes more neuronal progenitor cells to exit the cell cycle and presumably drive more cells into neuronal fate.

\subsubsection{Analysis of the cleavage plane orientation of mitotic RGCs}

The increased differentiation rate into neurons, measured by cell cycle exit index together with the reduced self-renewal capacity of $M c p h 1^{\Delta / \Delta}$ neuronal progenitor cells suggest that deletion of Mcph1 may lead to a precocious differentiation. RGCs undergo symmetric and asymmetric cell divisions which determine the daughter cell fate. A switch from symmetric to asymmetric divisions can lead to premature differentiation which has consequences for the brain size (Fish et al., 2008). 
A
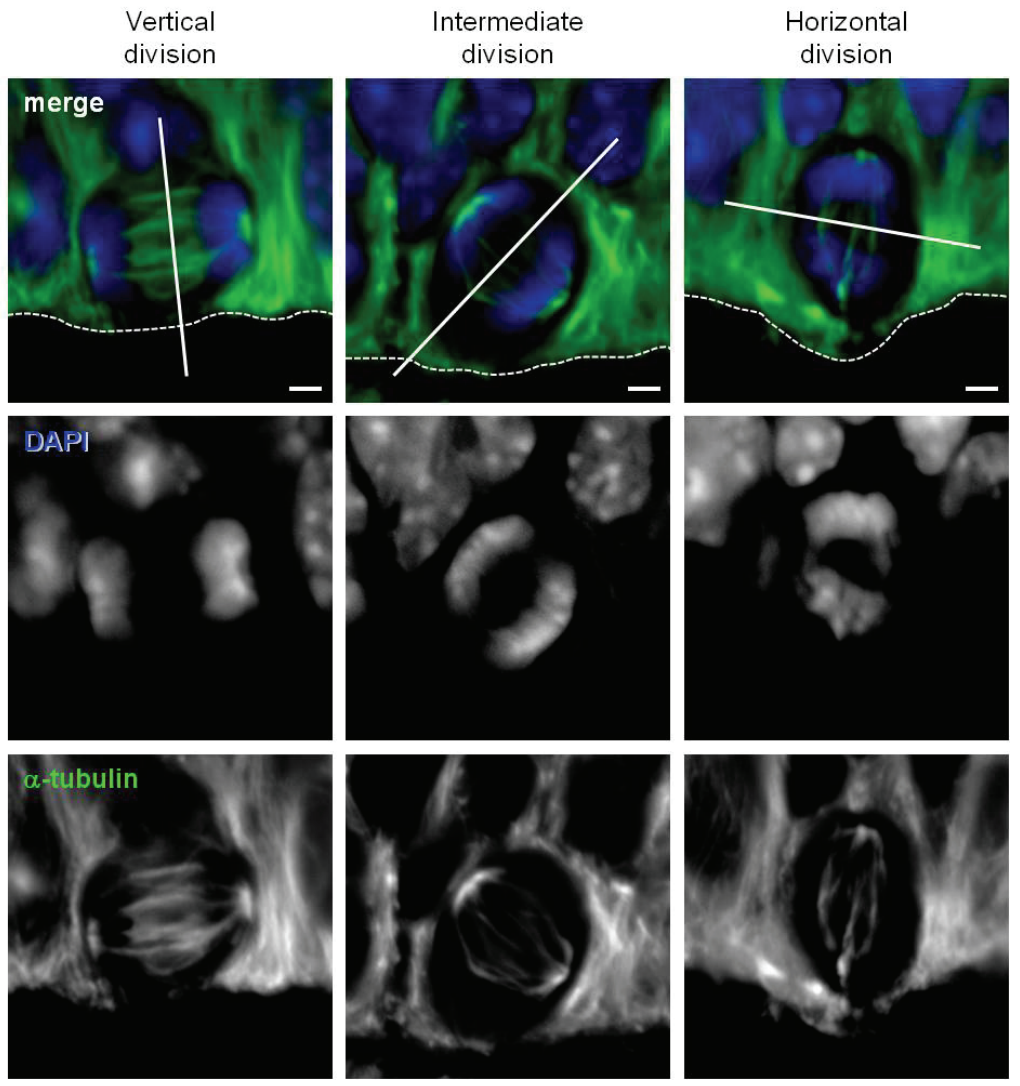

B

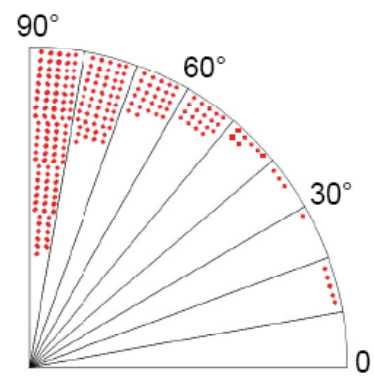

Mcph1ctr

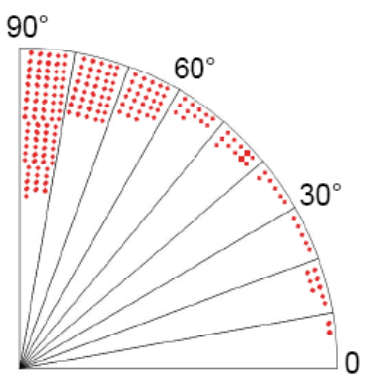

$\operatorname{Mcph} 1^{\Delta \Delta \Delta}$

Figure 24: $M c p h 1^{\Delta / \Delta}$ embryos show an increased number of RGCs undergoing cell division with an horizontal cleavage plane relative to the ventricular surface.

(A) E15.5 coronal brain sections were stained with spindle marker anti- $\alpha$-tubulin-antibody (green) and DNA was stained with DAPI (blue). Examples of anaphase RGCs in the telencephalon are shown which undergo cell division with vertical, intermediate or horizontal angle of the cleavage plane (solide white line) relative to the ventricular surface (dashed white line). Scale bars: $2 \mu \mathrm{m}$.

(B) The angles of the cleavage plane relative to the ventricular surface of RGCs were measured and classified into groups of $10^{\circ}$ ranging from $0^{\circ}$ (horizontal angle) to $90^{\circ}$ (vertical angle). Each red dot represents one anaphase or telophase RGC. Number of cells scored: $M c p h 1^{\text {ctr. }}: 217$ cells, Mcph $1^{\Delta / \Delta}$ : 194 cells. Chi $^{2}$-test was performed for statistical analysis. 
We therefore analyzed the ratio between symmetric and asymmetric cell divisions of RGCs in the dorsal telencephalon. It has been proposed that the mitotic cleavage plane of RGCs at the apical surface is important for the equal or unequal distribution of cellular components, hence defining symmetric and asymmetric cell divisions (Kosodo et al., 2004; Marthiens and ffrenchConstant, 2009). Cleavage planes that are orientated vertical to the apical surface (vertical divisions) result in symmetric, proliferative divisions. Whereas cleavage planes parallel to the apical surface (horizontal divisions) result in asymmetric neurogenic divisions, because apical components will be inherited by only one daughter cell (Kosodo et al., 2004; Marthiens and ffrench-Constant, 2009).

The cleavage plane of mitotic RGCs in anaphase or telophase was determined in E15.5 brain sections of $M c p h 1^{\Delta / \Delta}$ embryos and littermate control embryos. Cleavage planes of RGCs in metaphase were not included in the measurement, since the metaphase plane can still rotate prior to anaphase and can therefore not be used to define the cleavage plane (Haydar et al., 2003). Figure 24A shows examples of mitotic RGCs at E15.5 in anaphase or telophase in the dorsal telencephalon with vertical or horizontal divisions, as well as with an intermediate angle of the cleavage plane relative to the apical surface. The brain sections were stained with an antibody against $\alpha$-tubulin which helped to define the cleavage plane. The cleavage plane of anaphase or telophase RGCs was deduced from the orientation of the DAPI-stained sister chromatids (Figure 24A, solid white line) and the angle of the cleavage plane was measured relative to the apical surface (Figure 24A, dashed white line).

The resulting angles of the cleavage plane were grouped into 9 different groups of angles with a range of $10^{\circ}$ each group, from $0^{\circ}$ representing horizontal divisions (presumably symmetric) to $90^{\circ}$ representing vertical (presumably asymmetric) divisions. Figure 24B shows the distribution of the angles of the cleavage plane of RGCs division in the dorsal telencephalon of E15.5 embryos. Each red dot represent a single anaphase or telophase RGC. Mcph $1^{\Delta / \Delta}$ embryos show a reduced number of dividing RGCs with angles of the cleavage plane from $50^{\circ}$ to $90^{\circ}$ (162 cells, $83.5 \%$ ) compared with RGCs of Mcph $1^{\text {ctr }}$ embryos (201 cells, $92.6 \%$; Figure 24B). On the other hand, the number of dividing RGCs with an angle of cleavage plane ranging from $0^{\circ}$ to $49^{\circ}$ was increased in the Mcph1 $1^{\Delta / \Delta}$ embryos (32 cells, $16.5 \%$ ) compared with RGCs of Mcph1 ${ }^{\text {ctr }}$ embryos (16 cells, $7.4 \%$; Figure 24B). This shift of the cleavage plane of dividing RGCs from vertical angles to more horizontal angles suggests an increased number of asymmetric cell divisions of $M c p h 1^{\Delta / \Delta}$ RGCs. 


\subsubsection{Increased number of Mcph1-deficient mitotic RGCs bypassing the apical plasma membrane}

The change of orientation of the cleavage plane of RGCs may bypass the apical plasma membrane at the apical surface, which would lead to an unequal distribution of adherens junction complexes and apical components, an event leading to distinct daughter cell fate (Fish et al., 2008).

To further examine the possible bypass of the apical plasma membrane due to an increased number of dividing RGCs with a horizontal angle of the cleavage plane, we performed antibody staining against cadherin to visualize the cell membrane of dividing RGCs. The apical plasma membrane of RGCs is not stained by this antibody which reveals the contact of the cell to the apical surface, known as 'cadherin hole' (Kosodo et al., 2004), which consists of the Par protein complex (mPar3, Par6, PKC $\zeta$; Marthiens and ffrench-Constant, 2009). The cleavage plane of anaphase or telophase RGCs was deduced from the orientation of the DAPI-stained sister chromatids (Figure 25A, dashed white line), similar to the measurement of the cleavage plane orientation (chapter 3.4.2). Dividing RGCs were scored as equal or unequal distribution of the 'cadherin hole' according to the cleavage plane orientation (Figure 25A, yellow bars indicate the 'cadherin hole'). Figure 25B shows the percentage of anaphase or telophase RGCs with unequal distribution of the 'cadherin hole' at E14.5 which is considered to be an asymmetric cell division. Mcph $1^{\Delta / \Delta}$ embryos show an increased number of cells with such unequal distribution compared with $M c p h 1^{\text {ctr }}$ embryos at E14.5 (Figure 25B). A similar result was obtained when we analyzed the distribution of the 'cadherin hole' of dividing RGCs of E15.5 embryos (Figure 25C). These data indicate that a shift of the cleavage plane of Mcph $1^{\Delta / \Delta}$ RGCs allows only one daughter cell to possess the apical plasma membrane leading to an increased asymmetric cell division (Kosodo et al., 2004; Marthiens and ffrench-Constant, 2009). 
A
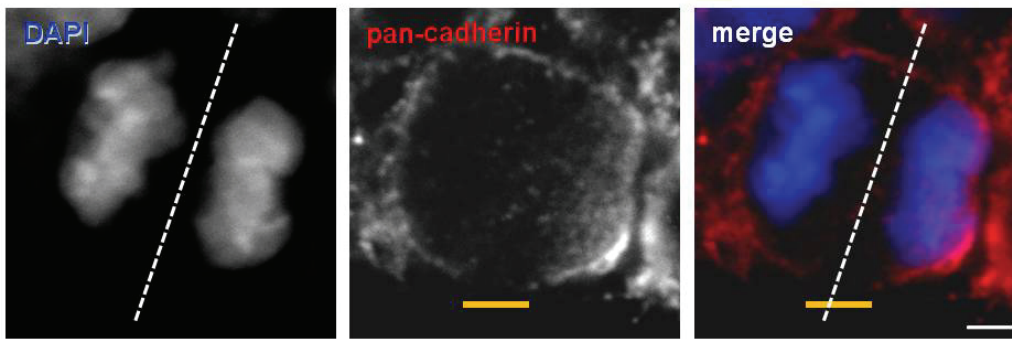

equal
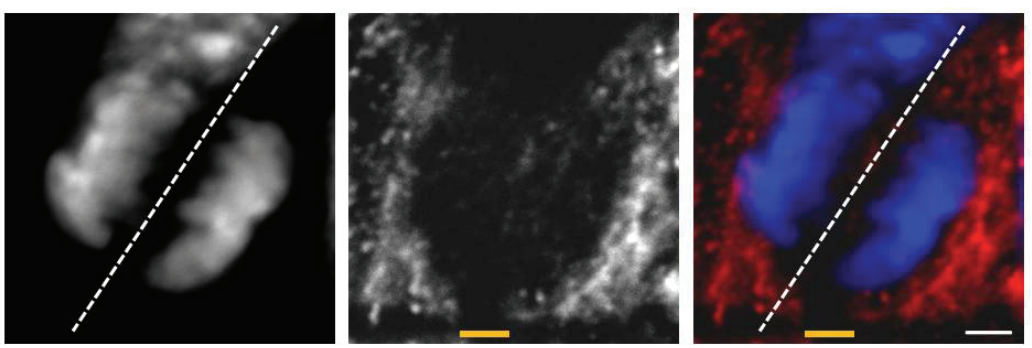

unequal

B

E14.5

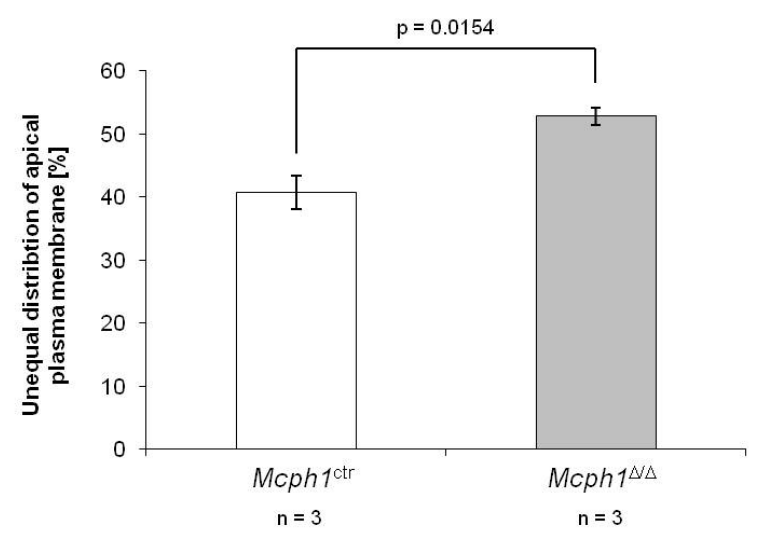

C

\section{E15.5}

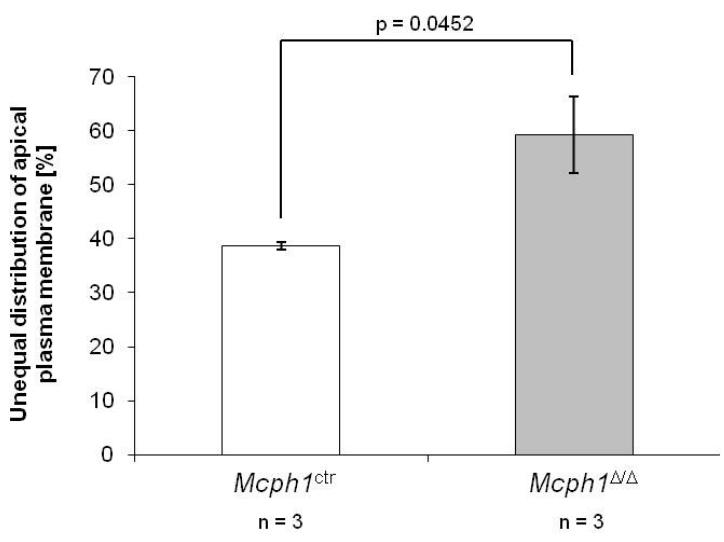

Figure 25: Increased number of $M c p h 1^{\Delta / \Delta}$ RGCs with unequal inheritance of the apical plasma membrane.

(A) E15.5 coronal brain sections were stained with pan-cadherin (red) which revealed the apical plasma membrane, the 'cadherin hole' (yellow line). The DNA was stained with DAPI to reveal the cleavage plane (dashed white line) of anaphase and telophase RGCs. Two examples of anaphase RGCs in the telencephalon are shown which either equally or unequally inherit the apical plasma membrane to the daughter cells. Unequal inheritance leads to distinct daughter cell fate (asymmetric cell division). Equal inheritance results in the same daughter cell fate (symmetric cell division). Scale bars: $2 \mu \mathrm{m}$.

(B) Quantification of the percentage of anaphase or telophase RGCs with unequal inheritance of the apical plasma membrane (asymmetric cell division) at E14.5.

(C) Quantification of the percentage RGCs in anaphase or telophase with unequal inheritance of the apical plasma membrane (asymmetric cell division) at E15.5.Means \pm standard error of means of $\mathrm{n}=$ number of embryos are shown. Number of anaphase or telophase RGCs: Mcph $1^{\text {ctr. }} 51$ cells (B) and 31 cells (C); Mcph1 ${ }^{\Delta / \Delta}: 53$ cells (B) and 34 cells (C). Student's t-test was used for statistical analysis. 


\subsubsection{Increased number of asymmetric cell divisions of Mcph1-deficient neuronal progenitor cells in vitro}

The change of orientation of the cleavage plane of RGCs and increased bypass of the apical plasma membrane of the $M c p h 1^{\Delta / \Delta}$ embryos argues for an alteration of the neuronal progenitor division mode. We next analyzed a possible shift of the neuronal progenitor cell division mode between symmetric and asymmetric cell divisions in vitro. To follow the fate of two daughter cells after cell division, we performed a Pair cell assay. The Pair cell assay was developed previously to detect the distribution of cell fate determinants into two daughter cells after neuronal progenitor cell division (Shen et al., 2002). In this study, we modified the Pair cell assay to analyze the daughter cell fate after neuronal progenitor cell division.

Neuronal progenitor cells were isolated from brains of E14.5 embryos and cultured for 7 days under conditions that prevent differentiation, to form primary neurospheres. On DIV7, neurospheres were dissociated and single cells were seeded at very low cell density (clonal density) on poly-D-lysine coated coverslips for adherent culture. Subsequently, cells were incubated for $24 \mathrm{~h}$ with medium which allows differentiation, and then cells were fixed for antibody-staining. During this incubation time, most of the neuronal progenitor cells divided once and thus formed a pair of cells, which represent the two daughter cells. Figure 26A shows an antibody-staining against progenitor marker Nestin (green) together with an antibody against neuronal marker $\beta$-tubulinllI (red) of the pair cells, which allowed us to identify the fate of these two daughter cells, hence conclusion of the type of neuronal progenitor division mode. The three possible types of division modes are shown in Figure 26A, which could be revealed by this antibody-staining: i) symmetric proliferative division generating two daughter progenitor cells (P-P, two Nestin-positive cells), ii) asymmetric division generating one daughter progenitor cell and one daughter neuron (P-N, one Nestin-positive cell and one $\beta$-tubulinlllpositive cell) and iii) symmetric neurogenic division generating two daughter neurons (N-N, two $\beta$-tubulinIII-positive cells). 
A
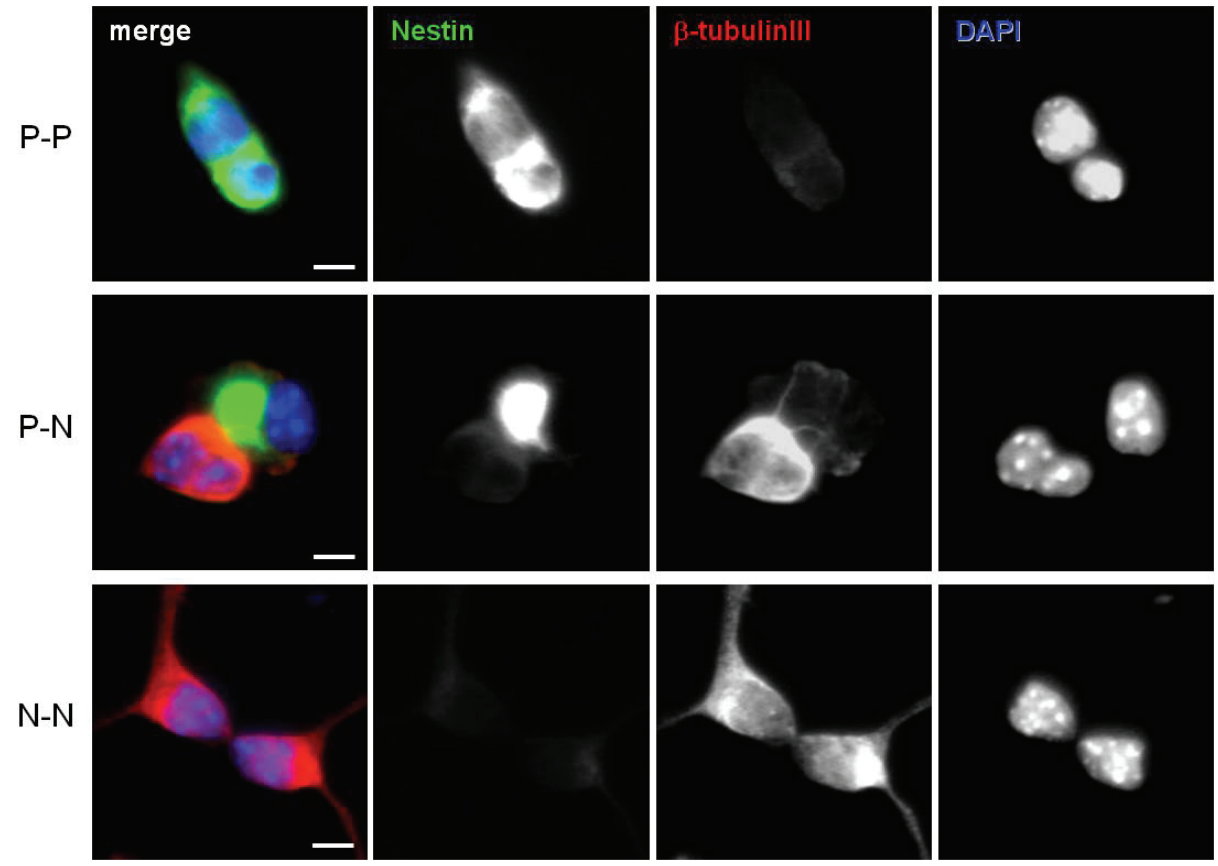

B

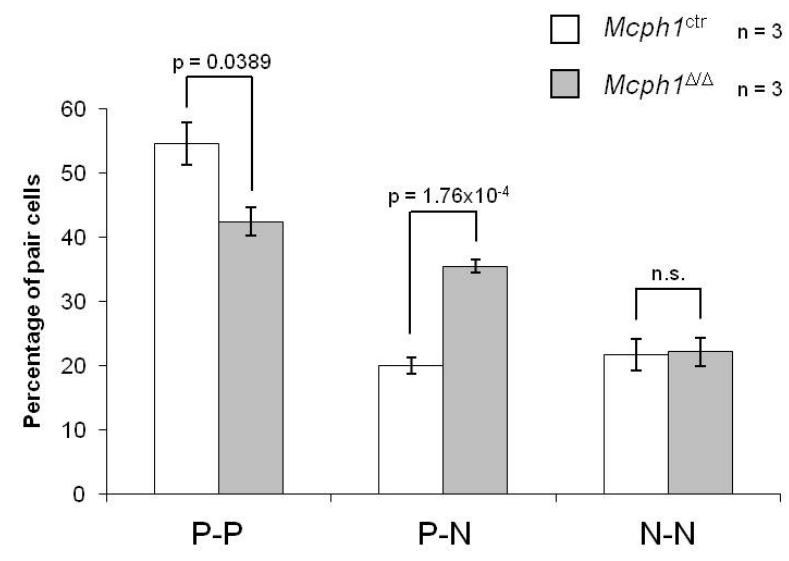

Figure 26: Pair cell assay shows increased asymmetric cell division of Mcph1-deficient neuronal progenitor cells in vitro.

(A) Neurospheres at 7DIV were dissociated, plated at clonal density and cultured for $24 \mathrm{~h}$ in differentiation medium. Neuronal progenitor cells which underwent cell division formed pair of daughter cells. Paired cells were stained with antibodies against neuronal progenitor marker nestin (green) and neuron marker $\beta$-tubulinlll (red) and DNA was stained with DAPI (blue). Examples show paired cells with two nestin-positive cells (P-P) which represent symmetric proliferative division and two $\beta$-tubulin daughter cells $(\mathrm{N}-\mathrm{N})$ represent symmetric neurogenic division. One nestin-positive and one $\beta$-tubulinpositive cell $(\mathrm{P}-\mathrm{N})$ represent asymmetric cell division. Scale bars: $5 \mu \mathrm{m}$.

(B) Quantification of the three possible cell division modes of the pair cell assay: symmetric proliferative division $(P-P)$, asymmetric division $(P-N)$ and symmetric neurogenic division $(N-N)$. Means \pm standard error of means of $n=$ number of embryos are shown. Student's t-test was used for statistical analysis. 
We next determined a possible alteration of the neuronal progenitor division mode after deletion of Mcph1 and therefore quantified the paired cells after antibody-staining and grouped them into P-P, P-N and N-N division modes (Figure 26B). We found that $M c p h 1^{\Delta / \Delta}$ neuronal progenitor cells showed a significant reduction of symmetric P-P division modes and on the other side an increase in asymmetric $\mathrm{P}-\mathrm{N}$ division mode compared to neuronal progenitor cells derived from Mcph1 $1^{\text {ctr }}$ embryos (Figure 26B). However, the neurogenic symmetric N-N division mode was very similar in both genotypes (Figure 26B).

From all these data, we conclude the deletion of Mcph1 leads shift in the balance of symmetric versus asymmetric cell division mode. Since the timing and the balance of these two types of division modes is crucial for the number of neurons generated during embryonic development, any disturbance would lead to an alteration to the final neuron number and hence the brain size (Fish et al., 2008).

\subsection{Mcph1-deletion leads to premature mitosis of neuronal progenitor cells caused by centrosomal loss of Chk1}

\subsection{1 $\mathrm{Mcph}^{\Delta / \Delta}$ neuronal progenitor cells show reduced centrosomal localization of Chk1 in G2-phase}

We next studied the molecular mechanism by which $\mathrm{MCPH} 1$ regulates the balance of symmetric and asymmetric cell division mode of neuronal progenitor cells. It has been reported previously that $\mathrm{MCPH} 1$ patient cells show a premature mitotic entry and premature chromosome condensation prior mitosis (Neitzel et al., 2002; Trimborn et al., 2004). It was hypothesized that $\mathrm{MCPH}$ might be due to defects in mitosis during neuronal progenitor cell divisions and disturbance in the mitotic progression can affect the neuronal progenitor division mode (Kaindl et al., 2010; Thornton and Woods, 2009; Woods et al., 2005). We therefore analyzed the specific role of MCPH1 during mitosis of neuronal progenitor cells. 
It was shown that the cell cycle regulator Chk1 has an important role in the transition from G2phase to M-phase (Loffler et al., 2006; Schmitt et al., 2006). For the undisturbed cell cycle progression, a specific sub-cellular fraction of the Chk1 kinase at the centrosomes is important. Centrosome associated Chk1 inhibits temporarily the Cdc25B phosphatase during interphase (Loffler et al., 2006; Schmitt et al., 2006), thereby controlling the activity of cyclinB1-Cdk1 complex (Kramer et al., 2004). Next we analyzed whether MCPH1 is required for the centrosomal localization of Chk1, since it has been reported that purified MCPH1 can directly bind to Chk1 in vitro (Alderton et al., 2006). In addition, MCPH1 was found to localize to the centrosomes in chicken DT40 cells and human lymphoblastoid cells as well as human U2OS cells (Jeffers et al., 2008; Zhong et al., 2006).

For this experiment, neuronal progenitor cells were isolated from E14.5 embryos and allowed to form primary neurospheres. Neurospheres were then synchronized by double thymidine block. Before release from the second thymidine block neurospheres were dissociated to single cell suspensions and plated on poly-D-lysine coated coverslips. Attached neuronal progenitor cells were fixed at different time points after the release to analyze cells in different cell cycle phases. The fixed cells were stained with antibodies against Chk1 and $\gamma$-tubulin as a centrosomal marker (Figure 27). First, we observed that in control neuronal progenitor cells, Chk1 is localized to the centrosomes in G2-phase (based on the presence of two centrosomes) in addition to its nuclear localization (Figure 27A, Mcph ${ }^{\text {ctr }}$, yellow arrows and insets). In prophase however, Chk1 is lost from the centrosomes presumably leading to activation of Cdc25B and cyclinB1-Cdk1 (Figure 27B, Mcph1 ${ }^{\mathrm{ctr}}$ ). Immediately after this, in metaphase cells, Chk1 is again associated with the centrosomes (Figure 27C). Interestingly, when we analyzed Mcph1-deficient neuronal progenitor cells, we found a significant number of cells in G2-phase in which Chk1 is lost from the centrosomes compared with control cells (Figure 27A, D). Whereas the Chk1 centrosomal localization in prophase as well as in metaphase was not affected by deletion of Mcph1 (Figure 27B, C, D). These results suggest that MCPH1 is required for the centrosomal localization of Chk1 specific in G2-phase of neuronal progenitor cells. 
A

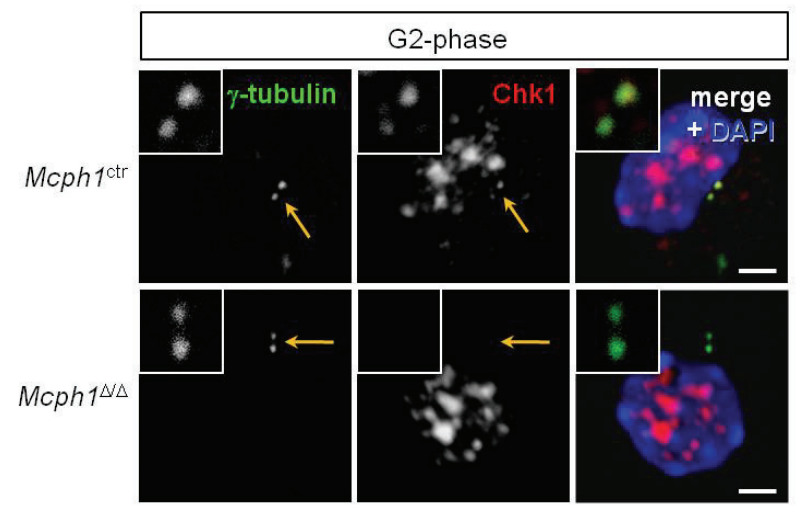

B

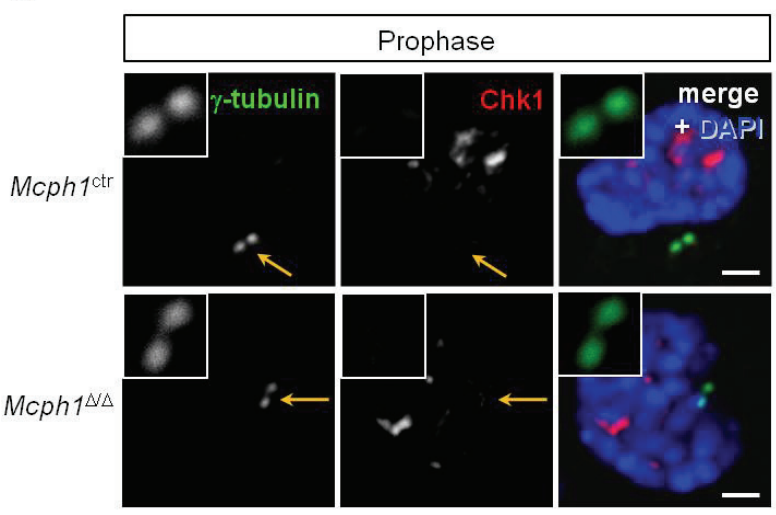

C

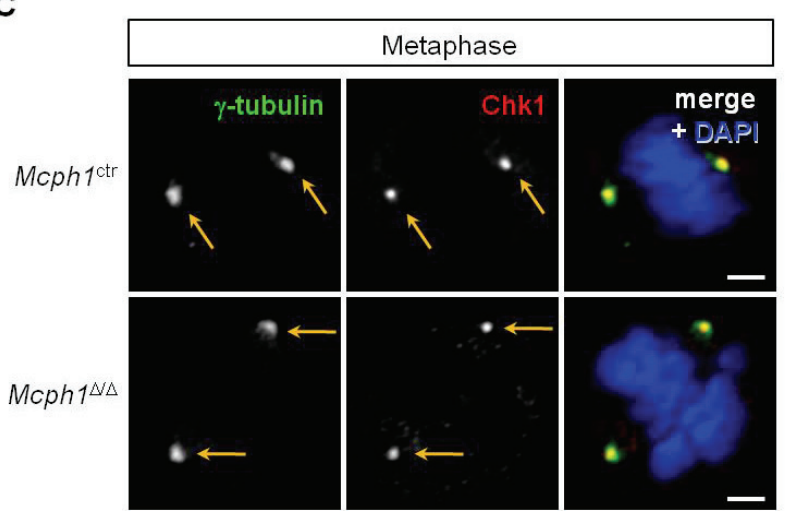

D

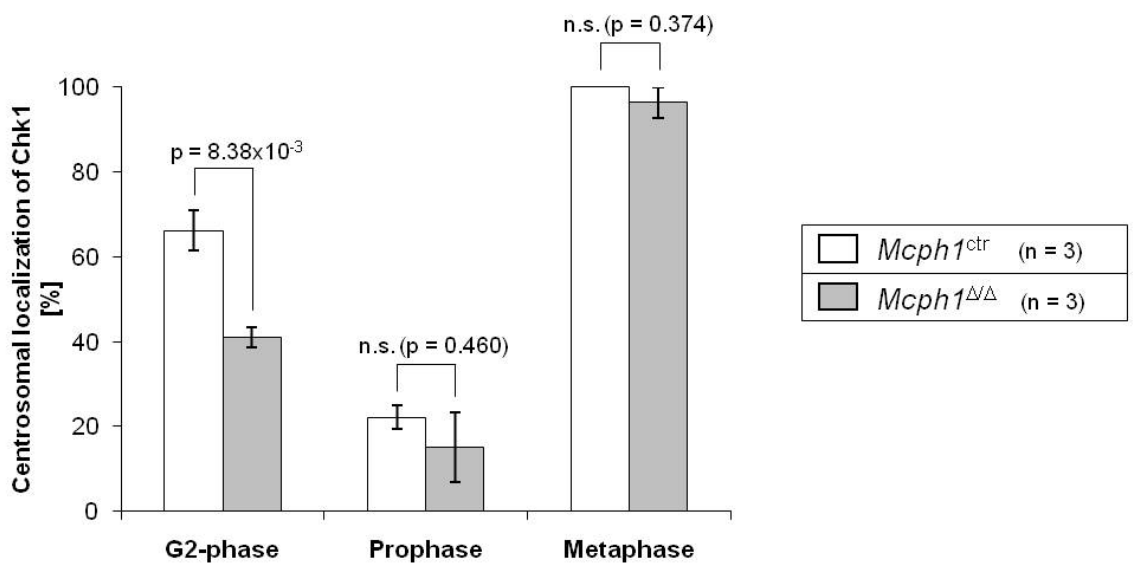

Figure 27: Chk1 is lost from the centrosomes in G2-phase of $M c p h 1^{\Delta / \Delta}$ neuronal progenitor cells.

(A, B and C) Neuronal progenitor cells at indicated cell cycle phases were stained with antibodies against centrosome marker $\gamma$-tubulin (green) and Chk1 (red) and DNA was stained with DAPI (blue). Arrows indicate the centrosomes, which are enlarged in insets. Scale bars: $2 \mu \mathrm{m}$.

(D) Quantification of the neuronal progenitor cells with centrosomal localization of Chk1 at indicated cell cycle phases. Means \pm standard error of means of $n=$ number of embryos are shown. Number of cells: Mcph $1^{\text {ctr }}: 91$ cells; Mcph1 $1^{\Delta \Delta}: 108$ cells. Student's t-test was used for statistical analysis. 


\subsubsection{Premature activation of Cdk1 in $M c p h 1^{\Delta / \Delta}$ neuronal progenitor cells}

Next we analyzed, whether the loss of Chk1 from the centrosomes in Mcph1-deficient neuronal progenitor cells in G2-phase would lead to a premature activation of Cdk1. The activation of Cdk1 was analyzed by a time course experiment after synchronization of neuronal progenitor cells in vitro. Neurospheres isolated from E14.5 embryos were synchronized 5 days after isolation by a double thymidine block, which arrests the cells in S-phase. The neurospheres were released from the cell cycle arrest and proteins were isolated at different time points after the release to follow the activation of Cdk1. Immunoblot analysis were then performed with an antibody against phosphorylated (inactive) Cdk1-Y18p (equivalent to human Y15-phosphorylation site). Figure 28 shows a representative immunoblot of synchronized neurospheres isolated from $M c p h 1^{\Delta / \Delta}$ and $M c p h 1^{\mathrm{ctr}}$ embryos at E14.5 after release from the thymidine block with an anti-Cdk1-Y18p antibody.

\begin{tabular}{|c|c|c|c|c|c|c|c|c|}
\hline \multicolumn{4}{|c|}{ Mcph1 $1^{\mathrm{ctr}}$} & \multicolumn{4}{|c|}{ Mcph1 $1^{\Delta \Delta}$} & \multirow[b]{2}{*}{$\mathrm{h}$ after TT-block release } \\
\hline 4 & 6 & 8 & 10 & 4 & 6 & 8 & 10 & \\
\hline & $=$ & $=$ & $=$ & - & $=$ & -0 & $\underline{-1}$ & Cdk1-Y18p \\
\hline & & & & & & & & $\beta$-actin \\
\hline 1.0 & 0.86 & 0.59 & 0.48 & 0.40 & 0.26 & 0.20 & 0.17 & \\
\hline
\end{tabular}

Figure 28: Premature Cdk1 activation of synchronized Mcph1-deficient neuronal progenitor cells.

Representative immunoblot of synchronized neurospheres with anti-Cdk1-Y18p and anti- $\beta$-actin antibodies. Neurospheres were synchronized by double thymidine block and proteins were isolated 4, 6 , 8 and 10 hours after release from the block. Numbers below the immunoblot represent signal intensities of Cdk1-Y18p after normalization to $\beta$-actin and relative to 4 hours of $M c p h 1^{\text {ctr }}$. 
Neurospheres of both genotypes showed a progression of Cdk1 activation (dephosphorylation) during the time after release from the cell cycle arrest in S-phase indicating a progression into mitosis. However, Mcph $1^{\Delta / \Delta}$ neurospheres showed a premature activation (dephosphorylation) of Cdk1 compared with $M c p h 1^{\text {ctr }}$ neurospheres. Already at 4 hours after the release from the double thymidine block Mcph1-deficient neurospheres showed a similar level of Cdk1-Y18p as the Mcph $1^{\text {ctr }}$ neurospheres at 10 hours after the release (Figure 28). We conclude that in Mcph1-deficient neuronal progenitor cells the loss of Chk1 from the centrosomes leads to a premature activation of Cdk1 and presumably to an early mitotic entry.

\subsection{3 $\mathrm{Mcph}^{\Delta / \Delta}$ neuronal progenitor cells show hypercondensed metaphase chromosomes}

The hallmark of MCPH1 patient cells is a premature chromosome condensation before M-phase and a delayed chromosome decondensation after mitosis (Neitzel et al., 2002; Trimborn et al., 2004). It was shown that constitutive active nuclear cyclinB1-CDK1 in human cells can promote premature chromosome condensation (Jin et al., 1998). We therefore asked, whether the mouse $M c p h 1^{\Delta / \Delta}$ neuronal progenitor cells show chromosomal condensation defects during mitosis presumably as a result of premature Cdk1 activation. To analyze the chromosomes of this cell type, we isolated neuronal progenitor cells from E14.5 embryos and allowed them to form primary neurospheres in vitro. At DIV7, the neurospheres were treated with colcemid to arrest the cells in metaphase and the metaphase chromosomes were scored for chromosome length. Microscopic analysis revealed a dramatic hypercondensation of the chromosomes of $M c p h 1^{\Delta / \Delta}$ neuronal progenitor cells compared with cells isolated from Mcph $1^{\text {ctr }}$ embryos (Figure 29A). The measurement of the length of all chromosomes per cell confirmed the observation of hypercondensed chromosomes in cells isolated from $M c p h 1^{\Delta / \Delta}$ embryos compared with cells from Mcph1 $1^{\text {ctr }}$ embryos (Figure 29B). This result shows that mouse neuronal progenitor cells derived from $M c p h 1^{\Delta / \Delta}$ embryos also display chromosome hypercondensation defects similar to that of human MCPH1 patient cells (Neitzel et al., 2002; Trimborn et al., 2004). 
A

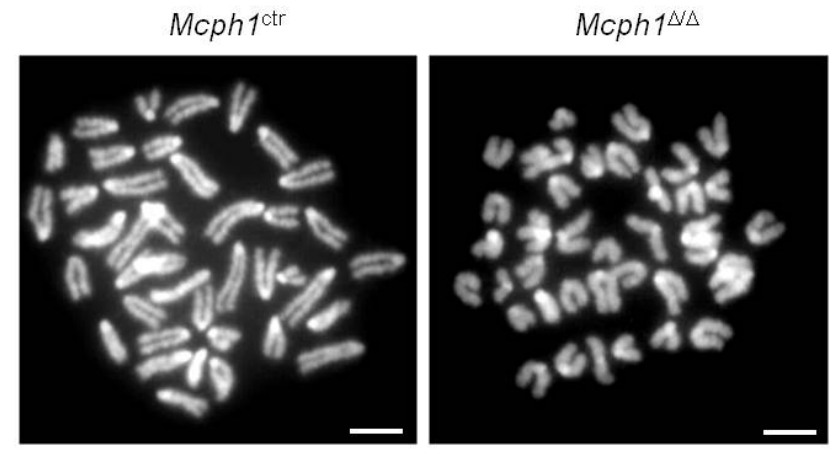

B

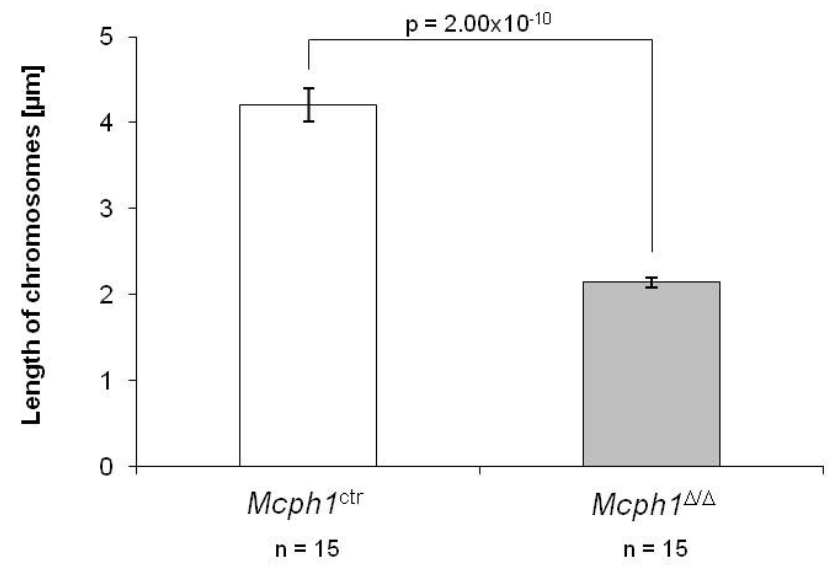

Figure 29: Mcph1-deficient neuronal progenitor cells show hypercondensed metaphase chromosomes.

(A) Examples of DAPI-stained metaphase chromosomes of neuronal progenitor cells isolated from embryos of indicated genotypes. Scale bars: $5 \mu \mathrm{m}$.

(B) Measurement of the length of all chromosomes per neuronal progenitor cell. Means \pm standard error of means of $n=$ number of metaphase cells. Student's t-test was performed for statistical analysis. 


\subsection{Uncoupling of the cell cycle from the centrosome cycle results in spindle alignment defects and apoptosis}

\subsection{1 $\mathrm{Mcph}^{\Delta / \Delta}$ neuronal progenitor cells show premature mitotic entry}

Cdk1 activation is the key event in regulation of the mitotic entry, we thus analyzed the consequences of premature Cdk1 activation in $M c p h 1^{\Delta / \Delta}$ neuronal progenitor cells. We first analyzed whether Mcph1-deletion results in an increased number of mitotic cells in an unsynchronized population of neuronal progenitor cells (mitotic index). To this end, we dissociated neurospheres isolated from E14.5 embryos and plated them on coated coverslips before fixation and antibody-staining. Figure 30A shows an example of a mitotic neuronal progenitor cell in vitro after antibody-staining against mitosis marker $\mathrm{pH} 3$. The staining revealed an increased number of $\mathrm{pH} 3$-positive cells of $M c p h 1^{\Delta / \Delta}$ genotype compared with Mcph $1^{\text {ctr }}$ cells (Figure 30B). Indeed, when we quantified the mitotic cells (pH3-positive), we found an increased mitotic index of $M c p h 1^{\Delta / \Delta}$ neuronal progenitor cells (Figure 30C).

Second, we tested whether the increased mitotic index of $M c p h 1^{\Delta / \Delta}$ neuronal progenitor cells is due to a premature mitotic entry. We therefore synchronized Mcph $1^{\text {ctr }}$ and Mcph $1^{\Delta / \Delta}$ neurospheres by double thymidine block to arrest cells in S-phase. Before the final release from the arrest, the neurospheres were dissociated and the single cell suspension was transferred to coated coverslips for adherent culture. At 6 hours after the release Mcph $1^{\Delta / \Delta}$ cells already showed prominent hypo-phosphorylated Cdk1 (26\% versus $86 \%$ in the Mcph1 ${ }^{\text {ctr }}$ cells, Figure 28), adherent neuronal progenitor cells were fixed and scored for mitotic cells by antibody-staining against the mitosis marker phosphorylated (S10p)-Histone3 (pH3). The quantification of pH3-positive cells revealed that Mcph1-deficient neuronal progenitor cells already at 6 hours after release from S-phase arrest showed an increased number of mitotic cells compared with $M c p h 1^{\text {ctr }}$ cells (Figure 31A). The increased number of mitotic cells at this time point together with the premature Cdk1 activation in a time course experiment (Figure 28) shows an early mitotic entry of $M c p h 1^{\Delta / \Delta}$ neuronal progenitor cells. 
A
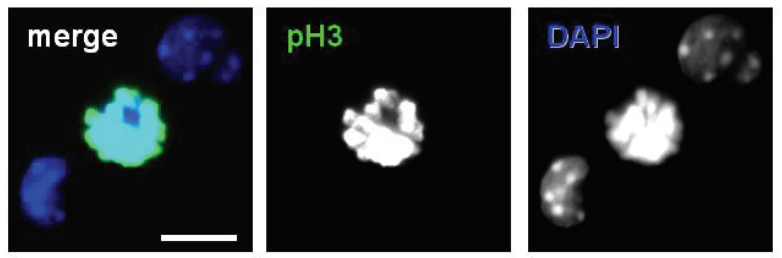

B

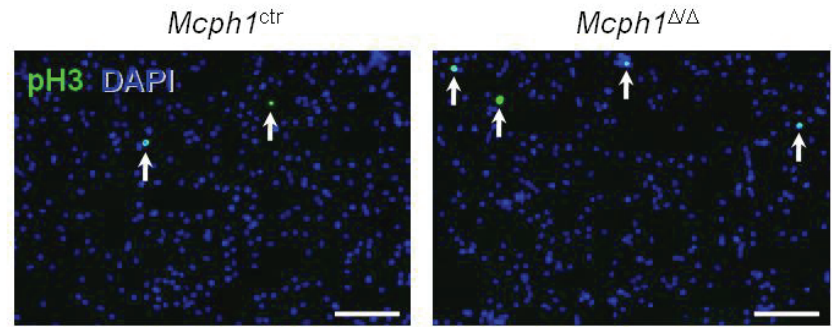

C

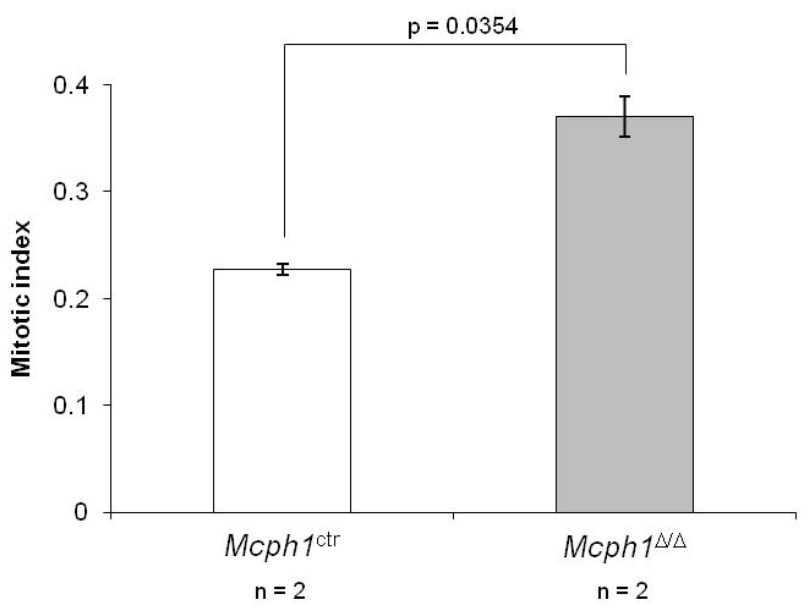

Figure 30: Higher mitotic index of Mcph1-deficient neuronal progenitor cells in vitro.

(A, B and C) Unsynchronized neuronal progenitor cells isolated from E14.5 embryos after neurosphere dissociation were stained with antibody against phosphorylated (S10p)-Histone3 (pH3, green) and counterstained with DAPI (blue).

(A) Examples of a pH3-positive (mitotic) and $\mathrm{pH} 3$-negative neuronal progenitor cells at high magnification. Scale bar: $10 \mu \mathrm{m}$.

(B) Examples of neuronal progenitor cells after staining of $\mathrm{pH} 3$ and DAPI of the indicated genotypes at low magnification. Scale bars: $100 \mu \mathrm{m}$

(C) Mitotic index. pH3-positive neuronal progenitor cells were quantified in an unsynchronized population and presented as percentage of DAPI-positive cells. Means \pm standard error of means are shown of $\mathrm{n}=$ number of embryos. Student's t-test was performed for statistical analysis. Mcph $1^{\text {ctr. }}$ : 7304 DAPI-positive cells and $M c p h 1^{\Delta / \Delta}: 7479$ DAPI-positive cells were scored. 


\subsubsection{The centrosome cycle of $M c p h 1^{\Delta / \Delta}$ neuronal progenitor cells lags behind the mitotic progression}

The entry into mitosis requires cellular events which have to be tightly controlled in time. One example of such event is the maturation of the centrosomes. The maturation of the centrosomes is controlled by the centrosome cycle that is linked to the cell cycle. Deletion of Mcph1 in neuronal progenitor cells results in an early mitotic entry. We therefore asked whether this would lead in addition to an early maturation of the centrosomes. To address this question, we used a centrosomal marker which labels specific mature centrosomes. Outer dense fiber 2 (ODF2), also known as cenexin, is a major component of sperm tail cytoskeleton and in addition is a component of centrosomal scaffold that is associated with the appendages of the mother centrioles (Ishikawa et al., 2005; Petersen et al., 1999). Hence, interphase cells exhibit only one mature ODF2-positive centriole. However, at the G2/M-transition the centriole maturation occurs and the second centriole acquires ODF2 (Lange and Gull, 1995). Thus, the acquisition of ODF2 marks the maturation of the centrioles and was therefore used as marker for mature centrosomes in neuronal progenitor cells.

Neuronal progenitor cells isolated from $M c p h 1^{\Delta / \Delta}$ and $M c p h 1^{\text {ctr }}$ E14.5 embryos were arrested by double thymidine block in S-phase and 6 hours after release from the block, fixed for antibody-staining. Figure 31C shows an antibody-staining of neuronal progenitor cells in G2phase (based on the presence of duplicated centrosomes) against ODF2 and the centrosome marker $\gamma$-tubulin. It shows a high magnification of the duplicated centrosomes of one cell with asymmetric ODF2-labeling which indicates an immature daughter centrosome and of one cell with symmetric ODF2-labeling showing a mature daughter centrosome (Figure 31C). Neuronal progenitor cells with mature centrosome (symmetric ODF2-labeling of centrosomes) at 6 hours after release from the S-phase block were quantified and revealed no difference in centrosomal maturation between $M c p h 1^{\Delta / \Delta}$ and $M c p h 1^{\text {ctr }}$ cells (Figure 31B), despite at the same time point after release from the S-phase arrest, more $M c p h 1^{\Delta / \Delta}$ neuronal progenitor cells entered mitosis (Figure 31A). Thus, we conclude that deletion of Mcph1 leads to a premature mitotic entry with an uncoupling of the mitotic progression from the centrosome cycle of neuronal progenitor cells. 
A

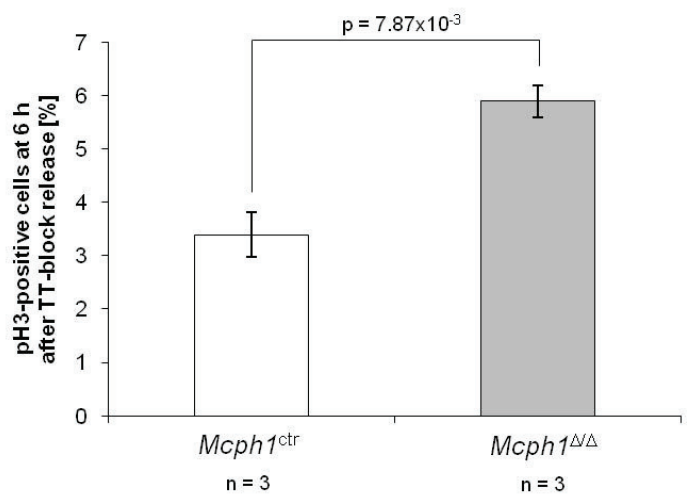

B

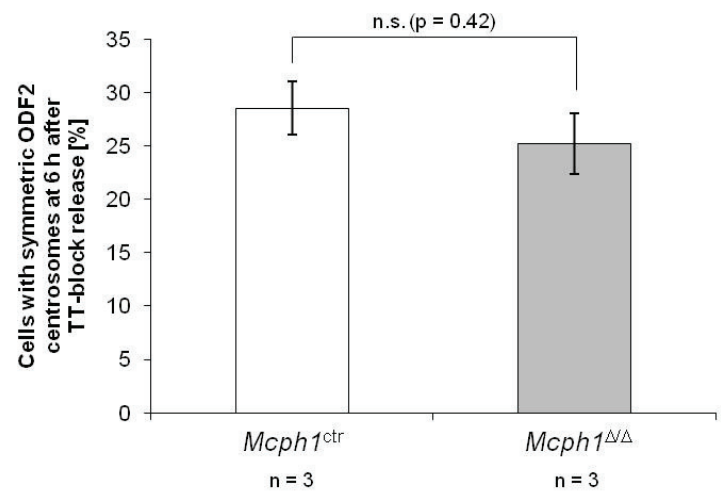

C

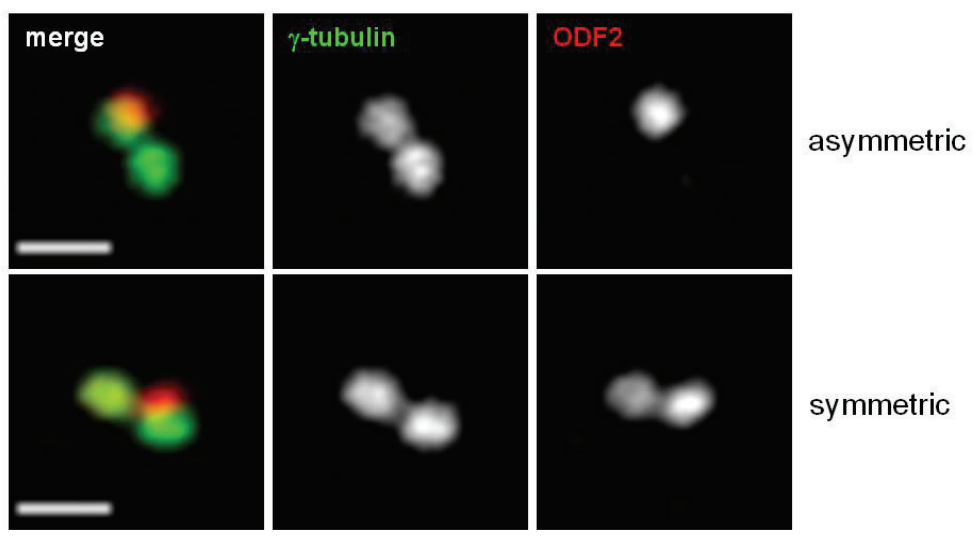

Figure 31: Uncoupling of the mitotic progression from the centrosome maturation in Mcph1-deficient neuronal progenitor cells.

(A) Quantification of mitotic neuronal progenitor cells at 6 hours after release from double thymidine block. Mitotic cells were scored as phosphorylated ( $\mathrm{S} 10 \mathrm{p})$ Histone3 (pH3)-positive cells and shown as

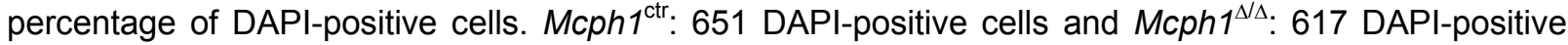
cells were scored.

(B) Quantification of neuronal progenitor cells with mature daughter centrosomes at 6 hours after thymidine block release. Cells with mature daughter centrosomes were scored as centrosomes with symmetric ODF2-staining (mature centrosome marker) and shown as percentage of DAPI-positive cells. $M c p h 1^{\text {ctr }}: 76$ DAPI-positive cells and $M c p h 1^{\Delta / \Delta}: 75$ DAPI-positive cells were scored.

(C) Examples of centrosomes ( $\gamma$-tubulin, green) of neuronal progenitor cells with asymmetric and symmetric ODF2-staining (red). Scale bars: $1 \mu \mathrm{m}$.

Means \pm standard error of means of $n=$ number of embryos are shown. Student's $t$-test was performed for statistical analysis. 


\subsubsection{Mitotic progression with immature centrosome results in mitotic spindle defects of Mcph1-deficient neuronal progenitor cells}

Although centrosomes are not essential for spindle assembly in mammalian cells (Hinchcliffe et al., 2001; Khodjakov et al., 2000), their function is probably to organize spindle poles which ensures high fidelity of chromosome segregation (Heald et al., 1997). A premature mitotic entry with a relative delay of centrosome maturation might cause a less efficient organization capacity of the mitotic spindle poles. Therefore, we tested whether Mcph1-deficient neuronal progenitor cells have defects in spindle formation.

Metaphase neuronal progenitor cells after adherent culture were stained with antibodies against pericentrin (centrosomal marker) and against $\alpha$-tubulin to label the mitotic spindles (Figure 32A, B). In Figure 32A one example of a normal metaphase neuronal progenitor cell of $M c p h 1^{\text {ctr }}$ origin is shown. However, $M c p h 1^{\Delta / \Delta}$ neuronal progenitor cells have a significant higher number of metaphase cells with misalignment of the mitotic spindles (Figure 32B). Indeed, the quantification revealed that a dramatic number of metaphase $M c p h 1^{\Delta / \Delta}$ neuronal progenitor cells showed defects of the mitotic spindles compared with Mcph $1^{\text {ctr }}$ cells (Figure 32C). Notably, the defects in the spindle alignment were restricted to bipolar defects, suggesting that deletion of Mcph1 does not lead to hyperamplification of centrosomes in neuronal progenitor cells. Indeed, when we quantified the number of centrosomes per cell in a population of unsynchronized neuronal progenitor cells, we did not observe cells with supernumerary centrosomes (data not shown). 
A

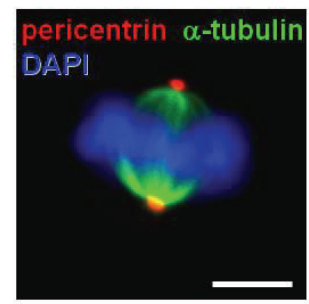

B

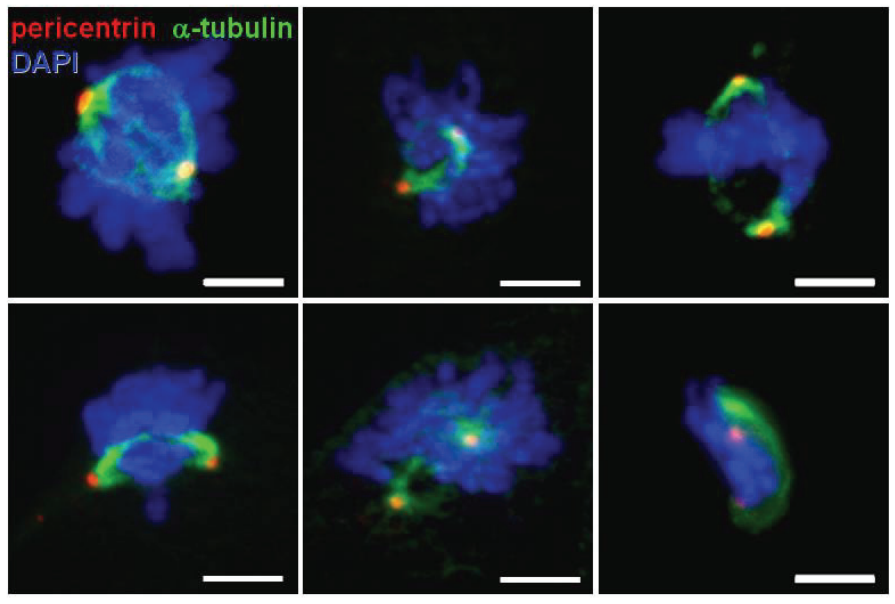

C

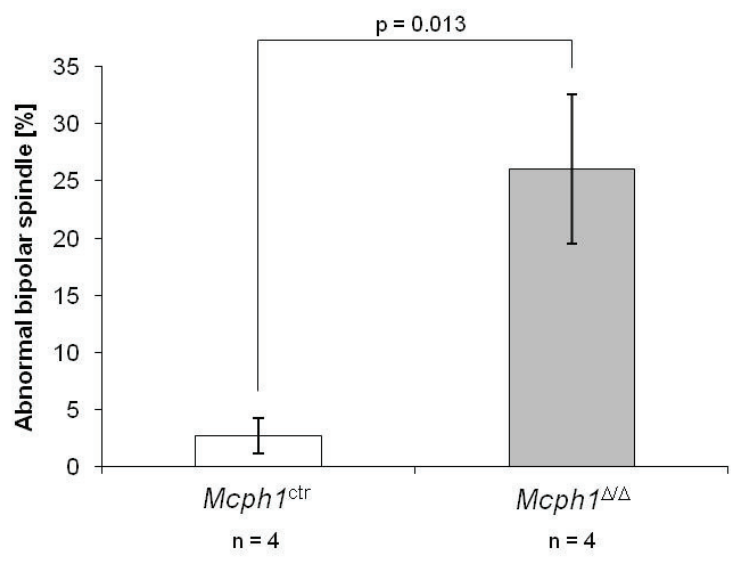

Figure 32: Mitotic spindle defects of Mcph1-deficient neuronal progenitor cells.

( $A$ and $B$ ) Examples of metaphase neuronal progenitors after antibody-staining against centrosome marker pericentrin (red) and spindle marker $\alpha$-tubulin (green) and DAPI-staining (blue). A normal metaphase cell is shown in A and $M c p h 1^{\Delta / \Delta}$ metaphase cells with aberrant spindles are shown in $\mathrm{B}$. Scale bars: $5 \mu \mathrm{m}$.

(C) Quantification of metaphase neuronal progenitor cell with aberrant mitotic spindles. Means \pm standard error of means of $n=$ number of embryos are shown. Student's t-test was performed for statistical analysis. 


\subsubsection{Mitotic spindle defects and mitotic catastrophe in the VZ of $M c p h 1^{\Delta / \Delta}$ embryos}

Premature entry into mitosis and defects in the alignment of the mitotic spindle can lead to cell death by mitotic catastrophe. It was shown that a premature activation and consequently nuclear transfer of the cyclinB1-Cdk1 complex is sufficient to cause apoptosis (Castedo et al., 2004; Heald et al., 1993; Porter et al., 2003). Since we observed an increased number of apoptotic cells in VZ and SVZ, the proliferative areas of the embryonic brains in Mcph $1^{\Delta / \Delta}$ embryos (chapter 3.2.5), we hypothesized that the apoptosis is caused by mitotic spindle defects and mitotic catastrophe of neuronal progenitor cells.

First, we analyzed whether the spindle defects can also be observed for RGCs in the VZ of embryonic brains during neurogenesis. When we stained brain sections of E13.5 embryos with an antibody against $\alpha$-tubulin, we found indeed cells with aberrant mitotic spindles and misaligned chromosomes. In Figure 33A one example of a mitotic RGC with defects in the alignment of the chromosomes in the VZ of a $M c p h 1^{\Delta / \Delta}$ embryo is shown. This defect is similar to the observed mitotic defects of cultured neuronal progenitor cells.

Next we analyzed whether the aberrant mitotic spindles and chromosome misalignment of Mcph1-deficient neuronal progenitor cells would lead to cell death by mitotic catastrophe in the VZ of the dorsal telencephalon. Mitotic catastrophe is considered as a type of cell death resulting from mitotic failure and is accompanied by caspase activation and DNA degradation (Castedo et al., 2004). We therefore performed a TUNEL assay on embryonic brain sections combined with an antibody-staining against $\alpha$-tubulin which helped us to identify mitotic cells undergoing cell death. The Mcph $1^{\Delta / \Delta}$ embryos showed cells in the VZ, presumably RGCs, which formed mitotic spindles (green) and were TUNEL-positive (red; Figure 33B). Two examples of such apoptotic cells due to mitotic failure of $M c p h 1^{\Delta / \Delta}$ embryos are shown in

Figure 33B. We did not detect this type of mitotic failure in the brain sections of Mcph1 $1^{\text {ctr }}$ embryos. Hence, we conclude that the increased cell death occurring during neurogenesis of $M c p h 1^{\Delta / \Delta}$ is at least in part the result of premature mitotic entry of neuronal progenitor cells which may lead in severe cases to mitotic failure and mitotic catastrophe. 
A
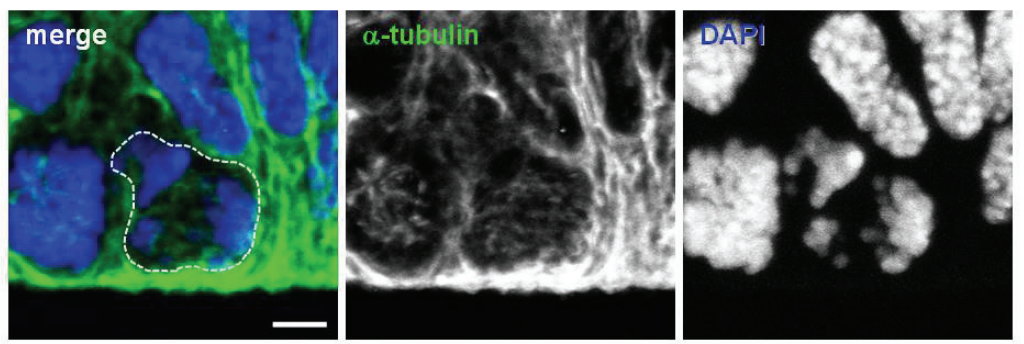

B
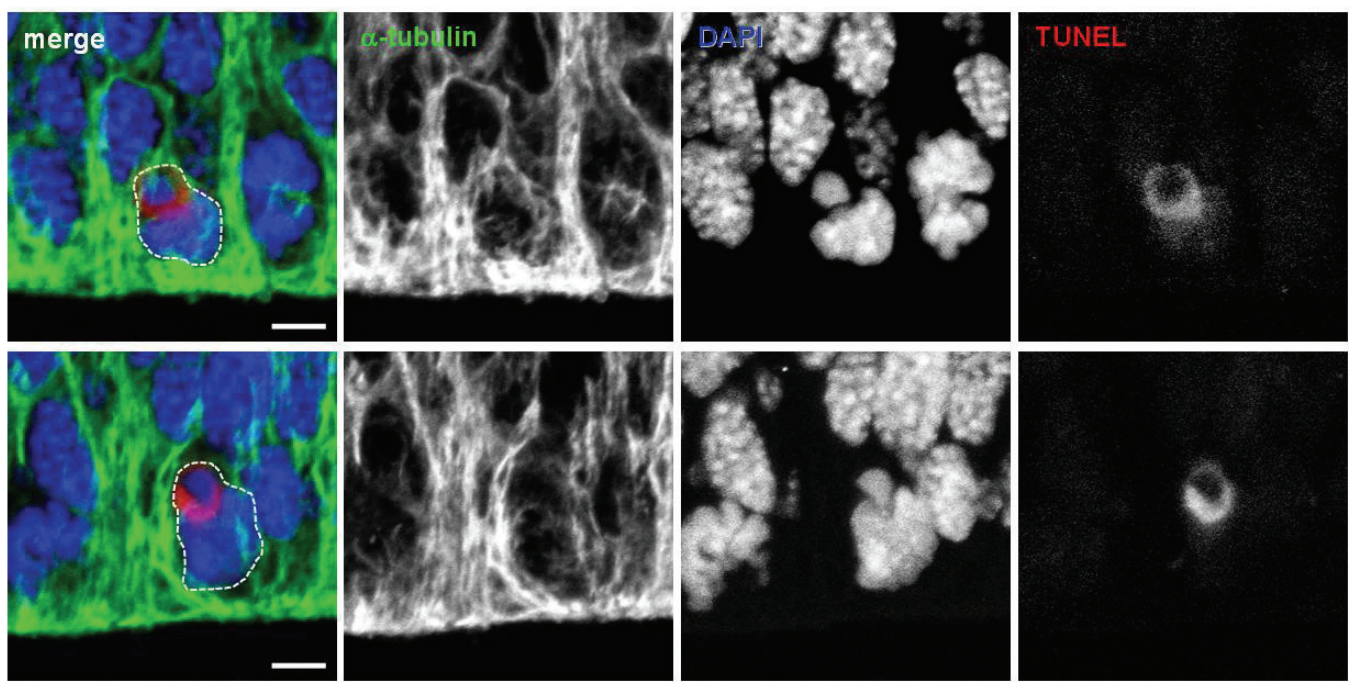

Figure 33: Mitotic spindle defects and mitotic catastrophe in VZ of $M c p h 1^{\Delta / \Delta}$ embryos.

(A) $M c p h 1^{\Delta / \Delta}$ coronal brain cryosections of E13.5 embryos were stained with spindle marker anti- $\alpha$-tubulin antibody (green) and DNA was stained with DAPI (blue). One example of a RGC with mitotic spindle defects and chromosome misalignment is shown. Dashed white line indicates the cell body. Scale bar: $5 \mu \mathrm{m}$.

(B) TUNEL assay (red) was performed on E13.5 coronal brain cryosections together with anti- $\alpha$-tubulin antibody-staining (green) and DAPI-staining (blue). Two examples of RGCs are shown which undergo mitotic catastrophe. Dashed white lines indicate the cell bodies. Scale bars: $5 \mu \mathrm{m}$. 


\subsection{Chk1 and MCPH1 function in the same pathway to regulate mitotic entry}

\subsubsection{Knockdown of Chk1 leads to aberrant mitotic spindles in MEFs}

The centrosomal localization of Chk1 in G2-phase of $M c p h 1^{\Delta / \Delta}$ was impaired. To further study whether MCPH1 and Chk1 indeed function in the same pathway in regulating the mitotic entry, we performed knockdown experiments. Due to low transfection efficiency of neuronal progenitor cells, MEFs were used in the following experiments.

A

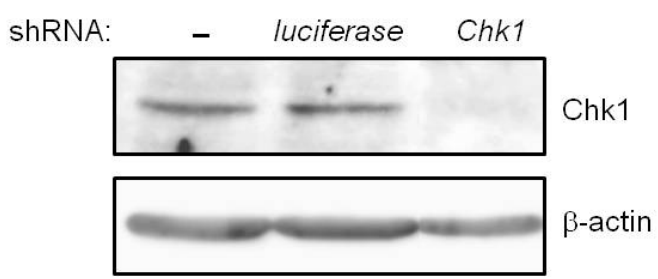

B

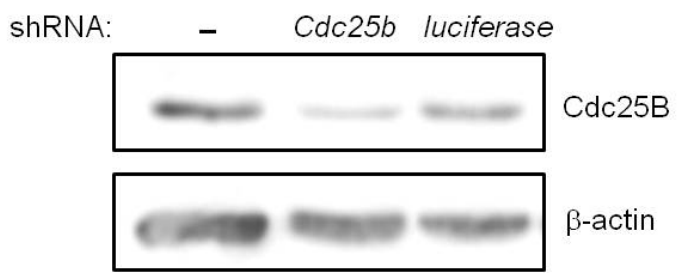

Figure 34: shRNA-mediated knockdown of Chk1 and Cdc25b in MEFs.

(A) Representative immunoblot of MEFs with antibody against Chk1. MEFs were transfected with EGFP-shRNA-vectors against Chk1 and luciferase-control and proteins were isolated $48 \mathrm{~h}$ later after sorting of GFP-positive cells. An antibody against $\beta$-actin was used as loading control.

(B) Representative immunoblot of MEFs with antibody against Cdc25B. MEFs were transfected with EGFP-shRNA-vectors against $C d c 25 b$ and luciferase-control and proteins were isolated $48 \mathrm{~h}$ later after sorting of GFP-positive cells. An antibody against $\beta$-actin was used as loading control.

MEFs were isolated from $M c p h 1^{+/+}$embryos as well as from $M c p h 1^{\Delta / \Delta}$ embryos at E13.5 and immortalized according to the 3T3-protocol. First, we analyzed whether knockdown of Chk1 in control cells would lead to aberrant mitotic spindles. To this end, we generated short hairpin 
RNA (shRNA) vectors against Chk1 and luciferase as control. Additionally, these vectors express the Egfp gene, encoding enhanced green fluorescent protein (EGFP) to trace the positively transfected cells. MEFs were then transfected with the EGFP-shRNA vectors by electroporation and after 48 h GFP-positive cells were sorted using FACsorting followed by protein isolation for immunoblot analysis. Figure 34A shows that the shRNA-vector against Chk1 can efficiently knockdown the expression of Chk1 in MEFs without any detectable protein level of Chk1.

To analyze mitotic cells after knockdown of Chk1, transfected MEFs were arrested in G0phase by contact inhibition for $48 \mathrm{~h}$. Since Chk1 is essential for mammalian development and viability in mice and cells (Liu et al., 2000; Takai et al., 2000) the G0-arrest would reduce the possible lethal effects of the Chk1 knockdown in MEFs. Additionally, the G0-arrest was used to synchronize the MEFs. Cells were released and 24 to $28 \mathrm{~h}$ later fixed to analyze mitotic cells by antibody-staining. To analyze spindle formation defects of GFP-positive cells, we used antibodies against centrosome marker pericentrin and spindle marker $\alpha$-tubulin and stained the DNA with DAPI (Figure 35). Since we detected different mitotic aberrations after Chk1 knockdown, we classified the defective metaphase cells into different groups as shown in Figure 35 and Table 4. We found that most untransfected Mcph1 $1^{\text {ctr }}$ metaphase MEFs (GFPnegative) showed normal bipolar spindles and only a minor population displayed either aberrant bipolar spindle or multipolar spindles, due to centrosome hyperamplification (Table 4). In contrast, untransfected $M c p h 1^{\Delta / \Delta}$ MEFs, which also served as control, showed an increased number of metaphases with abnormal bipolar spindles (Table 4). However, the number of cells showing multipolar spindles was not significantly increased in Mcph1-deficient MEFs compared to control cells, reminescent of the mitotic defects of Mcph1-deficient neuronal progenitor cells (Table 4 and Figure 32). Strikingly, we observed that knockdown of Chk1 in Mcph $1^{\text {ctr }}$ MEFs resulted in a high number of aberrant metaphase cells compared with control shRNA-luciferase transfected cells (Table 4). 

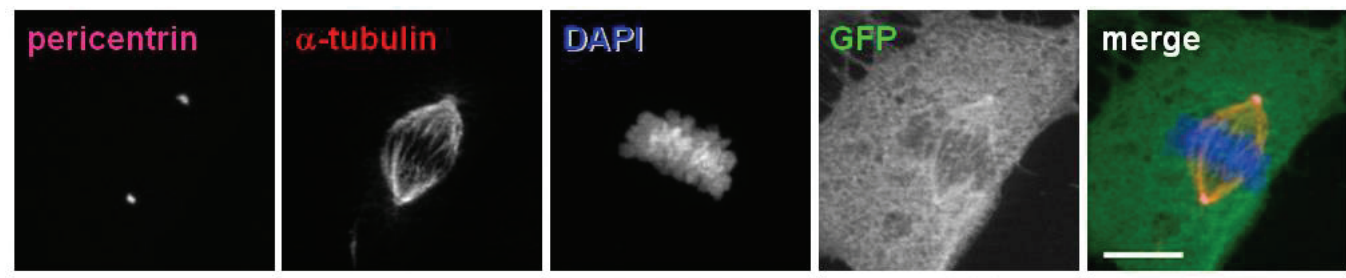

normal bipolar
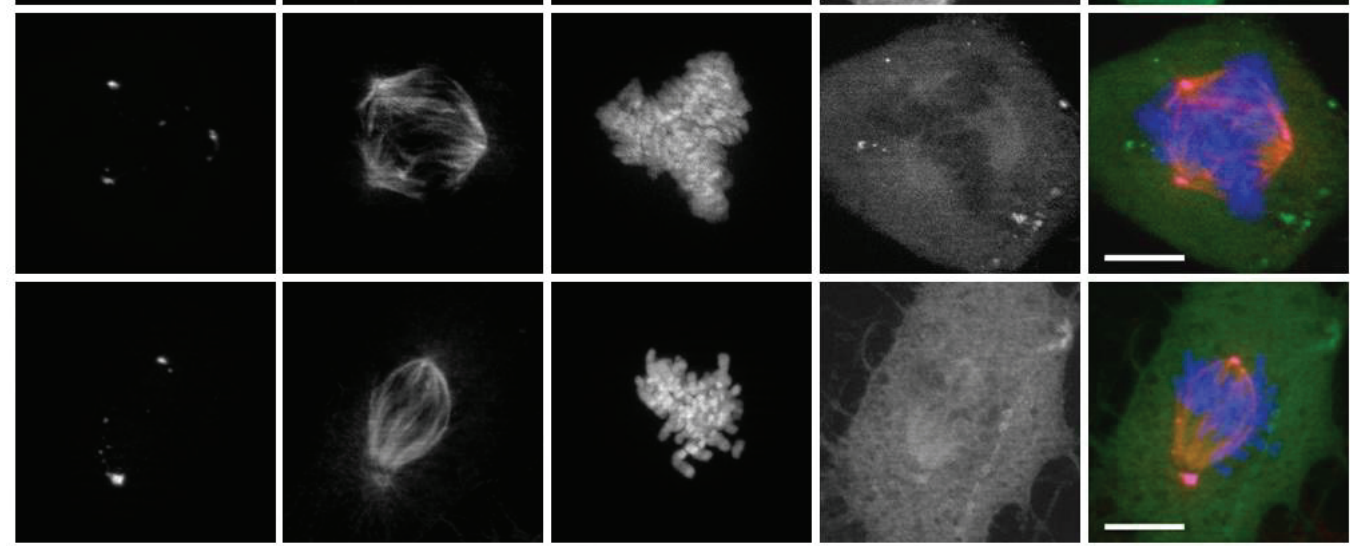

multipolar
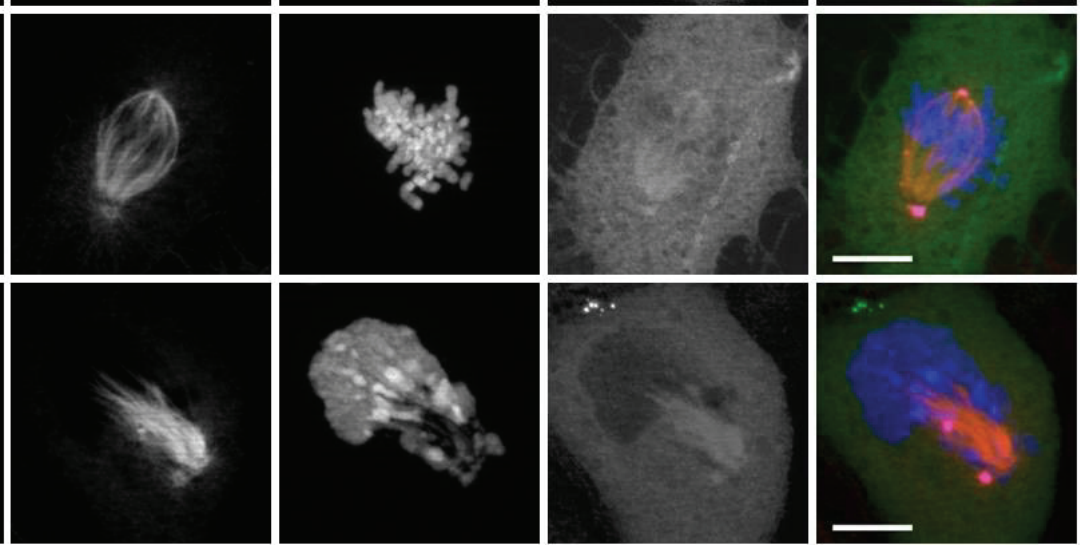

abnormal bipolar

abnormal bipolar, no chromosome condensation

Figure 35: Mitotic spindle aberrations of MEFs after EGFP-shRNA-vector transfection. 3T3-immortalized MEFs were transfected with EGFP-shRNA-vectors against luciferase, Chk1 or $C d c 25 b$ and kept at contact inhibition for $48 \mathrm{~h}$ to synchronize the cells. Cells were fixed 24 to $28 \mathrm{~h}$ after release from the contact inhibition to analyze mitotic cells. Fixed GFP-positive cells (green) were stained with antibodies against centrosome marker pericentrin (magenta) and spindle marker $\alpha$-tubulin (red) and DNA was stained with DAPI (blue). Examples of transfected MEFs with normal mitotic spindles or with indicated mitotic spindle defects are shown. Scale bars: $10 \mu \mathrm{m}$. See also Table 1 and Figure 36.

However, the spindle defects of Chk1 knockdown cells seemed different from the mitotic defects observed in the Mcph $1^{\Delta / \Delta}$ MEF cells. First, we found an increased number of cells with multipolar spindle after knockdown of Chk1, which is different to Mcph1-deleted cells (Table 4). Second, the cells with bipolar spindle defects resulting from Chk1 knockdown showed no chromosome condensation (Figure 35 and Table 4). We conclude that Chk1 together with $\mathrm{MCPH} 1$ as a co-factor has a critical role in the correct spindle assembly. 
However, knockdown of Chk1 leads to more pronounced spindle defects compared with $M c p h 1^{\Delta / \Delta}$ cells.

Table 4: Mitotic spindle morphologies of MEF cells after EGFP-shRNA-vector transfection. Means \pm standard error of means of three independent experiments are shown.

\begin{tabular}{|c|c|c|c|c|}
\hline & \multicolumn{3}{|c|}{ Mcph $1 \mathrm{ctr}$} & \multirow{2}{*}{ 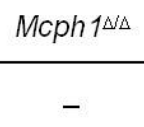 } \\
\hline shRNA: & - & luciferase & Chk1 & \\
\hline \multicolumn{5}{|l|}{ Spindle morphology } \\
\hline Normal bipolar [\%] & $85.4 \pm 2.6$ & $81.5 \pm 2.4$ & $8.3 \pm 1.6$ & $41.6 \pm 1.5$ \\
\hline Multipolar [\%] & $8.9 \pm 0.9$ & $8.6 \pm 2.5$ & $9.5 \pm 2.2$ & $9.8 \pm 3.8$ \\
\hline Aberrant bipolar [\%] & $5.7 \pm 2.1$ & $9.9 \pm 2.5$ & $6.8 \pm 3.4$ & $48.6 \pm 5.0$ \\
\hline $\begin{array}{l}\text { Aberrant bipolar; } \\
\text { no chromosome condensation [\%] }\end{array}$ & 0.0 & 0.0 & $63.7 \pm 6.2$ & 0.0 \\
\hline $\begin{array}{l}\text { Multipolar; } \\
\text { no chromosome condensation [\%] }\end{array}$ & 0.0 & 0.0 & $11.7 \pm 1.2$ & 0.0 \\
\hline
\end{tabular}

\subsubsection{Knockdown of Cdc25b corrects mitotic spindle defects of Chk1 knockdown and $M_{c p h 1^{\Delta / \Delta}}$ MEFs}

During unperturbed cell cycles, centrosomal Chk1 is known to control Cdk1 activation and mitotic entry (Kramer et al., 2004). This is achieved by temporary negative control of the Cdc25B phosphatase (Loffler et al., 2006). Hence, the underlying cause of the spindle defects in MEFs with downregulated Chk1 might be the deregulation of Cdc25B. To address this question, we generated an EGFP-shRNA-Cdc25b-vector to downregulate Cdc25b. The efficiency of the shRNA was tested by immunoblot with an antibody against Cdc25B after transfection of MEFs and FACsorting of GFP-positive cells. The EGFP-shRNA-Cdc25b-vector could efficiently downregulate the protein level of Cdc25B (Figure 33B). 
We analyzed mitotic MEFs after EGFP-shRNA-vector transfection similarly as described in chapter 3.6.1 and classified metaphase cells as normal bipolar spindles, multipolar spindles and aberrant bipolar spindles (examples are shown in Figure 34). For this experiment, we grouped all cells with bipolar spindle defects together irrespective of the chromosome condensation state. As similarly shown in Table 4, knockdown of Chk1 in Mcph1 $1^{\text {ctr }}$ cells causes metaphase cells with defective bipolar spindles as well as multipolar spindles compared with untransfected and control shRNA-luciferase transfected Mcph ${ }^{\text {ctr }}$ cells (Figure 36). When we transfected EGFP-shRNA-Cdc25b together with shRNA-Chk1 (without EGFP), the number of metaphase cells with aberrant bipolar spindles was significantly reduced compared with Chk1 knockdown cells (Figure 36). However, we observed that the number of cells with multipolar spindles was unaffected by additionally knockdown of Cdc25b in Chk1 knockdown Mcph1 $1^{\text {ctr }}$ cells (Figure 36). This suggests that the centrosome hyperamplification in Chk1 knockdown cells is likely not caused by deregulated Cdc25B in controlling mitotic entry.

Next we analyzed whether the bipolar spindle defects of $M c p h 1^{\Delta / \Delta}$ MEFs is similarly mediated by Cdc25B deregulation. The number of MEFs with aberrant bipolar spindles was increased in the $M c p h 1^{\Delta / \Delta}$ MEFs compared with Mcph $1^{\text {ctr }}$ MEFs and was not affected by transfection with the control shRNA-luciferase vector (Figure 36). Interestingly, downregulation of Cdc25B can significantly rescue a fraction of $M c p h 1^{\Delta / \Delta}$ cells with bipolar spindle defects (Figure 36). We conclude that the bipolar spindle defects of Chk1 knockdown MEFs are caused by deregulated Cdc25B. Hence, MCPH1 plays a role in the Cdc25B-Cdk1 pathway together with Chk1 to regulate mitotic entry and spindle assembly. 


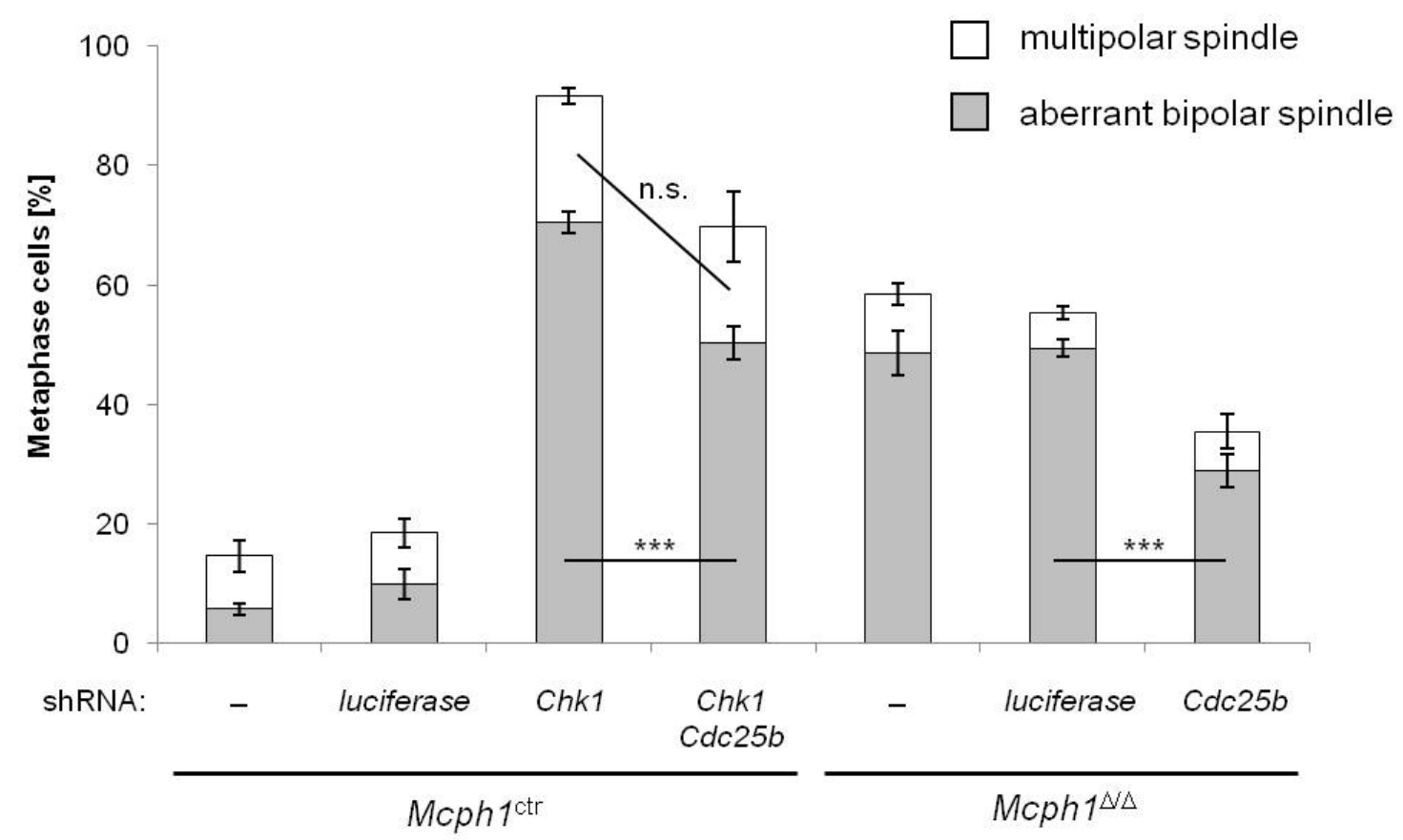

Figure 36: Rescue of mitotic spindle aberrations of MEFs by Cdc25b knockdown.

Quantification of metaphase 3T3-immortalized MEFs with aberrant spindles after transfection with EGFP-shRNA-vectors against luciferase, Chk1 or Cdc25b alone or with EGFP-shRNA-vector against Chk1 together with shRNA-vector against Cdc25b. GFP-positive cells were stained with antibodies against pericentrin and $\alpha$-tubulin and DNA was stained with DAPI to analyze mitotic cells with spindle aberrations. Examples are shown in Figure 34. Means \pm standard error of three independent experiments are shown. One-way ANOVA-test was performed for statistical analysis. ${ }^{* * *} p<0.001$; n.s. = not significant. 


\section{Discussion}

\subsection{Primary microcephaly of a MCPH1 mouse model}

$\mathrm{MCPH}$ is a disorder of defective prenatal development of the CNS and is characterized by a reduced brain size at birth (Woods et al., 2005). MCPH patients have a reduced brain size with normal architecture but the cerebral cortex shows the greatest size reduction. $\mathrm{MCPH}$ is caused by mutations in any of the seven MCPH genes leading to the subtypes MCPH 1 to 7 (reviewed in Kaindl et al., 2010; Woods et al., 2005).

We generated Mcph1 knockout mice and showed that these mice have a reduced brain size and thereby resembling the major human phenotype of $\mathrm{MCPH} 1$ patients. $M c p h 1^{\Delta / \Delta}$ mice have a reduced brain size at birth and the cerebral cortex shows the greatest reduction. The clinical definition of human $\mathrm{MCPH}$ is a reduced head circumference of more than two standard deviations below the age and sex matched average (Kaindl et al., 2010; Woods et al., 2005). The head circumference is used as a measurement of brain size. Although, other methods have been used, such as nuclear magnetic resonance imaging (NMRI), the measurement of head circumference remains a common and simple method to determine the brain size (Kaindl et al., 2010). We analyzed the brain weight of mice as a reference of the brain size and showed that deletion of Mcph1 in mice leads to a reduction to $69 \%$ compared with the brain weight of control mice at newborn stage. Although the reduction of the brain weight at this stage seems very dramatic, the brain of adult (P90) $M c p h 1^{\Delta / \Delta}$ mice showed a reduction to $89 \%$ compared with the brain weight of control mice. This might be explained by the postnatal development of the CNS in mice. The gliogenesis, the generation of astrocytes and oligodendrocytes, starts in late embryonic development and continues postnatally, which leads to an increase of the brain volume after birth (Kessaris et al., 2006; Mission et al., 1991). We hypothesize that in mouse, $\mathrm{MCPH} 1$ has functions in the embryonic neurogenesis but its role in gliogenesis might be of less importance.

Another explanation of the alleviated brain weight reduction of $M c p h 1^{\Delta / \Delta}$ adult mice compared with newborn mice would be a normal development of the cerebellum in the mutant mice. In mouse, the first neurons of the cerebellum leave the proliferative area around E11 to form a 
plate-like structure that develops into the cerebellum. But after birth, the cerebellum undergoes over a 1,000-fold increase in volume until the third postnatal week (reviewed in Goldowitz and Hamre, 1998). Deletion of Mcph1 in mice did not affect the cerebellar development (data not shown). Consistently, human MCPH patients do not show apparent reduction of the cerebellar cortex irrespective of the mutated gene (Guernsey et al., 2010; Kaindl et al., 2010; Kalay et al., 2011; Nicholas et al., 2010; Yu et al., 2010). This seems surprising, because the cerebellar development is controlled by neuronal progenitor cell proliferation, differentiation and neuronal migration similarly to the cerebral cortex development (Goldowitz and Hamre, 1998). The reasons why loss of $\mathrm{MCPH}$ protein function lead to reduction of the cerebral cortex, but does not affect cerebellar development are currently not understood, but might be due to a specific gene expression profile. Indeed, we found a high expression of Mcph1 in the dorsal telencephalon in close proximity to the lateral ventricle of mouse embryos, but not in the developing cerebellum of newborn mice (Z.W. Zhou, data not shown).

A specific role of $\mathrm{MCPH}$ genes in determining the cerebral cortex size would support a hypothesis of accelerated evolutionary changes of these genes that is coupled with an enlargement of the cerebral cortex. Since the mammalian cerebral cortex enhances the behavioral and cognitive abilities, such as perception, memories, thoughts and language, a bigger cortex would be an evolutionary advantage (Ponting and Jackson, 2005). Studies of the ASPM gene sequence showed that its evolution is significantly accelerated in great apes along the ape lineages to humans (Evans et al., 2004b). Similarly, MCPH1 protein sequence is highly accelerated throughout the lineage from simian ancestors to chimpanzees and humans. About 45 amino acid changes that seem advantageous have fixed during this evolution period (Evans et al., 2004a). Therefore, MCPH can be seen as a disorder of evolutionary regression since the brain size of MCPH patients resembles that of early hominids (Evans et al., 2004a; Wang and Su, 2004).

We found that deletion of Mcph1 in mice results in a sized reduction of the cerebral cortex, similar to human MCPH1, although with less severity. Given that humans have a 1,000-fold larger cortical surface area compared to rodents (Rakic, 2009), an about 20\% reduction of the cortical thickness in the $M c p h 1^{\Delta / \Delta}$ mice would amount to a great cortical size reduction in humans.

We further showed that $M c p h 1^{\Delta / \Delta}$ displayed a reduced body weight at newborn stage and in adulthood. Consistently, in certain $\mathrm{MCPH}$ cases a reduced body height and weight was 
reported (Passemard et al., 2009). Therefore, it is reasonable to hypothesize that the brain size reduction is secondary caused by a general whole body size reduction. However, since we observed a more dramatic reduction in the thickness of the cerebral cortex compared with other brain regions, argues against this hypothesis. Moreover, we deleted Mcph1 specific in the developing CNS of mouse embryos using the Cre-loxP-system. The Mcph1 ${ }^{\mathrm{CNS} \Delta}$ mice resembled the reduced brain weight of the conventional Mcph1 knockout mice, suggesting that the brain phenotype is not caused by extrinsic factors but rather due to defective MCPH1 functions in neuronal progenitor cells.

$M c p h 1^{\Delta / \Delta}$ mice, but not Mcph $1^{\mathrm{CNS} \Delta}$ mice display a reduced body weight. This suggests that the body size reduction is not caused by impaired brain functions. For example, the hypothalamus is fundamental in the control of the neuroendocrine system which affects hepatic glucose metabolism (for review see Kalsbeek et al., 2010). However, the reduced body weight of $M c p h 1^{\Delta / \Delta}$ seems rather be caused by defective MCPH1-dependent functions in other cell types or organs. Consistent with this, we found that $M c p h 1^{\Delta / \Delta}$ MEFs display mitotic defects similar to that of $M c p h 1^{\Delta / \Delta}$ neuronal progenitor cells.

We noticed that CNS-specific Mcph1-deleted mice showed a less severe reduction of the brain weight compared with $M c p h 1^{\Delta / \Delta}$ mice. This might be explained by the time point of the gene deletion using the nestin-Cre-transgene, which was shown to be expressed around E9.5 (Tronche et al., 1999; Zimmerman et al., 1994). Neuronal stem cells (NEs) undergo symmetric cell division to amplify the stem cell pool before the onset of neurogenesis around E10. Therefore, deletion of Mcph1 before E9.5, as in the conventional Mcph1 knockout mice, might affect the NE amplification through triggering apoptosis or by inducing a differentiated cell fate (see chapters 4.2 and 4.5) and thereby further reducing the stem cell number.

Human MCPH is characterized by mild to moderate mental retardation with an IQ between 30 and 80 (Cox et al., 2006; Woods et al., 2005). However, we were unable to detect deficits in the spatial learning and short term spatial memory of $M c p h 1^{\Delta / \Delta}$ mice. Hence, the Mcph1 knockout mice do not resemble fully the phenotype of human MCPH1 patients. The human cortex has a 1,000-fold larger cortical surface area compared with rodents (Rakic, 2009). The bigger human cortex generates behavioral and cognitive functions with higher complexity than the rodent cortex (Ponting and Jackson, 2005). Deletion of Mcph1 in mice may lead only to a 
very mild learning and memory impairment that lies out of the detectable range of the chosen behavioral test.

The mammalian cerebral cortex harbors primary and secondary motor cortices that are responsible for voluntary movements with the help of the basal ganglia, cerebellum and spinal cord (reviewed in Plowman and Kleim, 2010). However, the Mcph $1^{\Delta / \Delta}$ mice showed no defects in motor coordination, assessed by the Rotarod and balance bar tests. Consistently, motor coordination defects have not been described for any of the human MCPH patients. The reasons for the normal motor coordination despite the small cerebral cortex in the $\mathrm{MCPH}$ patients as well as in the Mcph1 knockout mice are currently unknown.

It is worth mentioning that two recent studies of Mcph1 knockout mice showed no microcephaly (Liang et al., 2010; Trimborn et al., 2010). The discrepancy is not known, but could be due to the technical approach of the gene disruption. Trimborn et al. used a gene trap approach to disrupt the mouse Mcph1 gene $\left(M c p h 1^{\text {gt/gt }}\right)$. The gene trap insertion site lies in intron 12 of Mcph1, presumably disrupting the C-terminal BRCT domains of the encoded protein (Trimborn et al., 2010). A possible truncated MCPH1 protein might contain the $\mathrm{N}$ terminal BRCT domain to partially fulfill functions. Another study by Liang et al. did not report a microcephaly phenotype, but found male and female infertility of Mcph1 knockout mice (Liang et al., 2010). The male mice were infertile due to defects in meiotic recombination. Interestingly, the $M c p h 1^{\Delta / \Delta}$ mice analyzed in the current study are also infertile (data not shown). But in contrast to the study by Liang et al., we assume the sterility of male Mcph $1^{\Delta / \Delta}$ might be caused by defects during early testis development. Since mice at newborn stage, before the onset of meiosis, exhibit a pronounced testis phenotype (data not shown). The gene disruption approach in the study of Liang et al. was the deletion of exon 2 of the Mcph1 gene (Liang et al., 2010). A possible internal transcription start site downstream of the deleted exon 2 may lead to the generation of a truncated protein isoform. We generated the Mcph1 knockout mice by disruption of exons 4 and 5 thereby generating several premature stop codons in exon 6 of the Mcph1 gene. Deletion of exons 4 and 5 presumably leads to a disruption of the first N-terminal BRCT domain. This might explains the phenotypic discrepancies to the other two Mcph1 knockout mice. However, due to the lack of reliable antibodies against mouse $\mathrm{MCPH} 1$ we were unable to rule out the possibility of truncated isoforms. 
Interestingly, cells isolated from the other two Mcph1 knockout mice show a chromosome condensation defect, reminiscent of $\mathrm{MCPH} 1$ patient cells, but without brain phenotype (Liang et al., 2010; Neitzel et al., 2002; Trimborn et al., 2004; Trimborn et al., 2010). A middle domain of human $\mathrm{MCPH} 1$ protein was shown to mediate the interaction with condensin II and this interaction seems important for chromosome condensation (Trimborn et al., 2006; Wood et al., 2008). It was further shown that the $\mathrm{N}$-terminal BRCT-domain of MCPH1 is needed for its centrosomal localization in chicken DT40 cells (Jeffers et al., 2008). Since the N-terminal BRCT-domain in the $M c p h 1^{\Delta / \Delta}$ mice is presumably non functional, we hypothesize that centrosomal functions of $\mathrm{MCPH} 1$ through its $\mathrm{N}$-terminal BRCT-domain play an important role during CNS-development. In summary, we demonstrated that deletion of Mcph1 in mice leads to primary microcephaly, resembling the brain phenotype of human MCPH1 patients.

\subsection{MCPH1 prevents unscheduled asymmetric cell divisions}

The cortical embryonic neurogenesis is restricted to a relatively short period during embryonic development (Dehay and Kennedy, 2007; Rakic, 1974). Thus, the production of neurons has to be tightly controlled in order to generate a sufficient number of neurons. An insufficient neuron production during neurogenesis can be caused by proliferation defects of neuronal progenitor cells. However, in vivo BrdU incorporation assays as well as in vitro primary neurosphere formation assays revealed that deletion of Mcph1 in mice has no impact on the proliferation of neuronal progenitor cells. Stem and progenitor cells are defined by their ability to self-renew and to differentiate. Deletion of Mcph1 compromises the self-renewal capacity of the neuronal progenitor cells and results in an increased differentiation, judged by the neuron production rate (cell cycle exit index). Thus, MCPH1 ensures the balance of self-renewal versus neuronal differentiation. It was shown by other studies that the shift in the balance of self-renewal and differentiation, without affecting cell cycle progression itself, can lead to premature depletion of the neuronal progenitor cell pool (De Pietri Tonelli et al., 2008; Silver et al., 2010). 
The self-renewal of progenitor cells ensures the maintenance of the progenitor cell pool. One of the mechanisms by which this is achieved is symmetric progenitor cell division. The symmetric cell division generates two identical daughter cells, whereas an asymmetric cell division gives rise to one stem cell and one differentiated progeny (reviewed in Knoblich, 2008). It has been proposed that the orientation of the cleavage plane of mammalian neuronal progenitor cells (NEs and RGCs, but not IPCs) is a determinant for the cell division mode. Similarly, the orientation of the cleavage plane of Drosophila melanogaster neuroblasts dictates the cell division mode (Fuerstenberg et al., 1998). Cleavage planes that are orientated perpendicular to the ventricular surface result in symmetric divisions, since apical and basal cellular components would be inherited equally by both daughter cells. An orientation of the cleavage plane horizontal to the ventricular surface leads to asymmetric cell division because apical components, such as the Par protein complex and junctional complexes, would be inherited by only one daughter cell leading to distinct cell fates (Gotz and Huttner, 2005; Knoblich, 2008). In NEs and RGCs, however, horizontal divisions are rare events (Landrieu and Goffinet, 1979; Smart, 1973). Since the apical plasma membrane of these cells constitutes a tiny fraction of the total plasma membrane, vertical dividing cells can bypass the apical plasma membrane leading to unequal inheritance of the apical components and asymmetric cell division (Kosodo et al., 2004; Marthiens and ffrench-Constant, 2009). Therefore the mechanisms controlling the cell division mode by regulating the orientation of cleavage plane must function with high accuracy. MCPH1 seems to be important to ensure such high accuracy of the cleavage plane, since $M c p h 1^{\Delta / \Delta}$ embryos show a significant increased number of RGCs bypassing the apical plasma membrane presumably leading to more asymmetric cell divisions compared with control embryos.

However, we hypothesize that MCPH1 does not regulate microtubule-dependent pushing or pulling forces to affect directly the orientation of the cleavage plane. An example of a protein with a direct function in the generation of microtubule-dependent forces is LIS-1, a member of the dynein complex. Mouse embryos mutant for the gene Lis-1, were shown to have a more dramatic increase of RGCs with horizontal cleavage planes compared with Mcph $1^{\Delta / \Delta}$ embryos (Yingling et al., 2008). Another example is LGN, a regulator of heterotrimeric G-proteins. Knockout embryos of Lgn displayed randomized cleavage plane orientations of RGCs (Konno et al., 2008). Since, Mcph $1^{\Delta / \Delta}$ RGCs do not show randomized cleavage plane orientations, we conclude that $\mathrm{MCPH} 1$ functions to prevent unscheduled asymmetric progenitor cell division by 
ensuring high accuracy of the cleavage plane orientation rather than affecting microtubuledependent forces to orientate the spindle poles.

RGCs are able to undergo two distinct types of asymmetric cell divisions, the direct neurogenic division and the division leading to the generation of one daughter RGC and one daughter IPC (lacopetti et al., 1999; Miyata et al., 2004; Noctor et al., 2004). However, not much is known of how the generation of IPCs is regulated. It was shown that some transcription factors act as cell fate determinants for IPCs, such as Tbr2 and Cux2 that are specifically expressed in IPCs (Cubelos et al., 2008; Englund et al., 2005). However, it seems that the specification of IPCs occurs in the RGCs before cell division, since the transcription factor AP2 $\gamma$ functions in RGCs to directly regulate the IPC fate determinants Tbr2 and Cux2 (Pinto et al., 2009). AP2 $\gamma$ is not expressed in IPCs itself, but rather in a subset of RGCs before the acquisition of IPC fate (Pinto et al., 2009). There is no evidence so far, that the orientation of cleavage plane of mitotic RGCs or the bypass of apical components are determinants for the asymmetric division leading to the generation of IPCs. Interestingly, Mcph $1^{\Delta / \Delta}$ embryos showed no defects in the generation of IPCs as analyzed by quantification of Tbr2-positive cells at E15.5 (data not shown). Since Mcph $1^{\Delta / \Delta}$ RGCs showed an increased number of cells bypassing the apical plasma membrane, it supports the hypothesis that the orientation of cleavage plane and bypass of apical components are dispensable for the generation of IPCs.

Differences in the cell cycle length could contribute to the $M c p h 1^{\Delta / \Delta}$ phenotype of increased asymmetric divisions (Gotz and Huttner, 2005). With the switch from symmetric proliferative divisions of NEs to neurongenic divisions of RGCs, the length of the cell cycle increases, particularly by increasing G1-phase (Takahashi et al., 1995). During neurogenesis both symmetric and asymmetric cell divisions co-exist. It was shown that at this stage, RGCs undergoing asymmetric neurogenic divisions have a longer cell cycle (Calegari et al., 2005). Artificially lengthening of the cell cycle of the G1-phase by drug-induced inhibition of Cdks in mouse whole embryo culture induced a premature neurogenesis (Calegari and Huttner, 2003). Conversely, shortening of G1-phase by overexpression of either cyclinD1-Cdk4 or cyclinD1 together with cyclinE1 using in utero electroporation in mouse embryos delayed neurogenesis (Lange et al., 2009; Pilaz et al., 2009). Hence, the cell cycle length, in particular the G1-phase length seems to be a determinant of symmetric proliferative and asymmetric neurogenic divisions of neuronal progenitor cells. Since we found that MCPH plays a role in the control of the cell cycle, in particular mitotic entry, we cannot rule out a contribution of cell cycle 
alterations in Mcph1-deficient neuronal progenitor cells to the neurogenesis defects of $M c p h 1^{\Delta / \Delta}$ embryos.

\subsection{Deletion of Mcph1 affects centrosomal maturation}

Defects in centrosomal maturation and/or activity can affect the mitotic spindle organization capacity. Does deletion of Mcph1 in mouse neuronal progenitor cells have any impact on the activity or maturation of centrosomes in neuronal progenitor cells? Strikingly, the gene products of Aspm (MCPH5) and Cdk5rap2 (MCPH3) were shown to localize to the centrosomes in mouse neuronal progenitor cells (Buchman et al., 2010; Fish et al., 2006). Although we were unable to detect the sub-cellular localization of MCPH1 in mouse neuronal progenitor cells due to technical limitations, we assume a centrosomal localization in this cell type as it was shown for other cell types, such as human U2OS, chicken DT40 and Drosophila melanogaster cells (Brunk et al., 2007; Jeffers et al., 2008; Rickmyre et al., 2007; Zhong et al., 2006).

The important function of centrosomal proteins for neurogenesis was demonstrated by studies on pericentrin, a protein of the pericentriolar matrix. Pericentrin functions in the $\gamma$-TuRC recruitment to the centrosomes and in mitotic spindle assembly (Doxsey et al., 1994; Zimmerman et al., 2004). Mutations in the human PCNT gene, encoding pericentrin, cause Seckel type 4 syndrome (Griffith et al., 2008) and microcephalic osteodysplastic primordial dwarfism type II (Rauch et al., 2008). Both disorders are characterized by microcephaly and mental retardation that are reminiscent of $\mathrm{MCPH}$. However, severe growth retardation has been reported for Seckel type 4 syndrome, whereas only some individuals of MCPH1 show growth retardation (Griffith et al., 2008; Passemard et al., 2009; Rauch et al., 2008). In addition, dwarfism was reported in MOPDIl patients (Rauch et al., 2008), but not in MCPH1 patients. Interestingly, it was shown that pericentrin interacts with CDK5RAP2 in cells isolated from brains of E11.5 mouse embryos and this interaction may play a role during neurogenesis (Buchman et al., 2010). Knockdown of PCNT in human U2OS cells results in depletion of 
centrosomal CDK5RAP2, suggesting that pericentrin recruits CDK5RAP2 to the centrosomes. Knockdown of PCNT in mouse embryonic brains by in utero electroporation phenocopies the defects of Cdk5rap2 knockdown such as premature differentiation of cortical neuronal progenitor cells into neurons (Buchman et al., 2010).

Moreover, the asymmetric inheritance of mother and daughter centrosomes is important for neurogenesis. The centrosomes duplicate once each cell cycle, resulting in the formation of two centrosomes. The centrosome retaining the older mother centriole is referred to as mother centrosome and the other that receives the new mother centriole is referred to as daughter centrosome (Doxsey, 2001). It has been shown that the centrosomal asymmetry is important for the spindle orientation of asymmetrically dividing Drosophila melanogaster neuroblasts (Rebollo et al., 2007; Rusan and Peifer, 2007). Consistently, during the peak phase of mouse neurogenesis, asymmetrically dividing RGCs inherit the mother centrosome to the progenitor daughter cell whereas the daughter centrosome is associated with differentiated cells (Wang et al., 2009). In vivo knockdown of ninein, encoding a protein specific for mature centrioles, leads to defects in the asymmetric inheritance of centrosomes and a premature depletion of neuronal progenitor cells (Wang et al., 2009). These studies underline the importance of centrosome function and maturation during neurogenesis which presumably affects the regulation of the critical balance between symmetric and asymmetric cell division of neuronal progenitor cells. The precise mechanism of cell division mode regulation by centrosomes is not completely understood.

We found that $M c p h 1^{\Delta / \Delta}$ neuronal progenitor cells prematurely entered mitosis, whereas the maturation of the centrosomes lagged behind. This uncoupling of the mitotic progression from the centrosome maturation results in an increased number of $M c p h 1^{\Delta / \Delta}$ neuronal progenitor cells undergoing mitosis with immature centrosomes. Similarly, asynchrony of nuclear and centrosome cycle has been hypothesized for Drosophila melanogaster mcph1 mutant cells, although no direct evidence was shown (Brunk et al., 2007). Immature centrosomes during mitosis may be unable to organize the spindle poles correctly leading to misaligned spindles (Thornton and Woods, 2009). Consistent with this, we observed an increased number of metaphase $M c p h 1^{\Delta / \Delta}$ neuronal progenitor cells as well as MEFs with defects in mitotic spindle alignment. As mentioned above, the mechanisms controlling the orientation of cleavage plane must function with high accuracy. Defects in spindle alignment are prone to bypass the apical 
plasma membrane leading to unequal inheritance of the apical components and asymmetric cell division (Kosodo et al., 2004; Marthiens and ffrench-Constant, 2009).

\subsection{MCPH1 functions in the centrosomal Chk1-Cdc25B pathway to regulate mitotic entry}

What is the molecular mechanism of $\mathrm{MCPH} 1$ to couple mitotic entry with the centrosome maturation? It may be through functions of $\mathrm{MCPH} 1$ in the gene transcription. It was shown by siRNA-mediated Microcephalin knockdown that MCPH1 regulates the expression of $\mathrm{CHK} 1$, BRCA1, RAD51, DDB2 and caspases in human cells through interactions with the transcription factor E2F1 (Lin et al., 2005; Xu et al., 2004; Yang et al., 2008). However, we found that $\mathrm{MCPH} 1$ is not required for the expression of Chk1 in mouse cells (data not shown). Similarly, previous studies demonstrated that deletion of Mcph1 in mice, as well as siRNAmediated knockdown in mouse cells has no impact on the gene expression of the reported genes (Trimborn et al., 2010). This suggests either distinct species-dependent functions of MCPH1 or off-target effects of the siRNA against human Microcephalin. To further support the hypothesis of unspecific off-target effects, Alderton et al. used another siRNA against MCPH1 in human cells and found no difference in the protein levels of Chk1 and BRCA1 (Alderton et al., 2006).

We found that Mcph1-deletion impaired, but not completely abolished the centrosomal localization of Chk1 in G2-phase of neuronal progenitor cells. Interestingly, in cells from another microcephaly disorder Seckel syndrome type 4, mutated in PCNT (Griffith et al., 2008), loss of Chk1 from the centrosomes has been reported (Tibelius et al., 2009). The requirement for both $\mathrm{MCPH} 1$ and pericentrin, encoded by $P C N T$, for the centrosomal localization of Chk1 might explain the similar phenotypes of patients with MCPH1 and Seckel syndrome type 4 . It is thus plausible that centrosomal localization of Chk1 is controlled by multiple interactions with several proteins, including $\mathrm{MCPH} 1$ and pericentrin and possible other centrosomal proteins. The MCPH3 protein CDK5RAP2 was also shown to interact with 
pericentrin at the centrosomes and this interaction is important for neurogenesis in mouse embryos (Buchman et al., 2010).

It is currently unknown how MCPH1 and Chk1 are recruited to the centrosomes. Up to date there is no data available on the localization of $\mathrm{MCPH} 1$ during the cell cycle. We were unable to analyze the sub-cellular localization in mouse neuronal progenitor cells, due to the lack of reliable antibodies against mouse $\mathrm{MCPH} 1$ protein. The Chk1 recruitment to the centrosomes seems to be independent of MCPH1, because Chk1 appeared in metaphase cells at high levels at the centrosomes in Mcph $1^{\Delta / \Delta}$ cells. However, MCPH1 may play a role in the removal of Chk1 from centrosomes in prophase. It has been demonstrated that centrosomal Chk1 is phosphorylated at S345 in prophase leading to immediate distribution of S345p-Chk1 throughout the cytoplasm (Wilsker et al., 2008), suggesting that Chk1-S345-phosphorylation is the signal for the Chk1 removal from the centrosomes. Unphosphorylated Chk1 is detected at the centrosomes shortly after in metaphase (Wilsker et al., 2008). The kinase responsible of Chk1-S345-phosphorylation at the onset of mitosis has not been identified. It was shown that after DNA damage, the ATR kinase phosphorylates Chk1 at multiple sites, including S345 leading to cell cycle arrest (Wilsker et al., 2008). It is not known whether ATR is responsible for the S345-phosphorylation without DNA damage. If this is the case, it would be a novel role of ATR in control of mitotic entry in the absence of DNA damage. Another possibility would be a kinase with a well established role in the control of mitotic events, such as Plk or AuroraA (Cazales et al., 2005; van Vugt and Medema, 2005). A possible role of MCPH1 in the regulation of upstream kinases of Chk1-S345-phosphorylation has to be determined.

Centrosomal Chk1 functions to prevent mitotic entry through inhibition of the phosphatase Cdc25B in interphase. This keeps Cdk1, the substrate of Cdc25B, in its phosphorylated inactive state (Kramer et al., 2004; Loffler et al., 2006; Schmitt et al., 2006). Interference of centrosomal localization of Chk1 in $M c p h 1^{\Delta / \Delta}$ G2-phase cells argues for a premature activation of Cdk1. Indeed, we found a premature Cdk1 activation in $M c p h 1^{\Delta / \Delta}$ neuronal progenitor cells. A premature activation of Cdk1 has been also suggested by a study using Drosophila melanogaster mcph1 mutant cells (Brunk et al., 2007).

We demonstrated that knockdown of Chk1 in wildtype MEFs resembled the mitotic defects of $M c p h 1^{\Delta / \Delta}$ MEFs and neuronal progenitor cells, thereby confirming the important functions of Chk1 in control of mitotic entry. Additional knockdown of Cdc25b can rescue the mitotic defects caused by Chk1 knockdown. This suggests that the mitotic defects due to Chk1 
deficiency are indeed caused by deregulation of its substrate Cdc25B. More importantly we found that knockdown of $C d c 25 b$ in $M c p h 1^{\Delta / \Delta}$ MEFs can rescue the mitotic defects in these cells, demonstrating that MCPH1 functions in the centrosomal Chk1-Cdc25B-pathway to regulate mitotic entry. Interestingly, knockdown of Chk1 leads to multipolar spindle poles, presumably due to centrosome hyperamplification. This phenotype was not observed in Mcph $1^{\Delta / \Delta}$ MEFs or neuronal progenitor cells. Knockdown of Cdc25b in Chk1 knockdown cells, specifically corrected bipolar spindle defects. This suggests that centrosomal hyperamplification in Chk1 knockdown cells is caused by deregulation of another Chk1dependent pathway that is independent of Cdc25B and MCPH1.

Moreover, knockdown of Chk1 leads to spindle defects in cells without condensed chromosomes. An explanation for this would be a specific function of MCPH1 in chromosome condensation that is independent of its control of mitotic entry. A hallmark of MCPH1 patient cells is a premature chromosome condensation prior to mitosis and a delayed decondensation after completion of cytokinesis (Neitzel et al., 2002; Trimborn et al., 2004). Consistently, in mouse $M c p h 1^{\Delta / \Delta}$ neuronal progenitor cells, we observed hypercondensed metaphase chromosomes. Since this defect is not observed in Chk1 knockdown cells, we conclude that the role of $\mathrm{MCPH} 1$ in regulation of chromosome condensation may be independent of its control of mitotic entry in the Chk1-Cdc25B-pathway.

\subsection{Cell death of Mcph1-deficient neuronal progenitor cells}

We found that at the end of neurogenesis the number of neuronal progenitor cells was reduced in the $M c p h 1^{\Delta / \Delta}$ embryos, suggesting progenitor cell attrition. We showed that apoptosis contributes to the loss of neuronal progenitor cells. What is the cause of apoptosis in Mcph1-deficient neuronal progenitor cells? It has been shown that defects in DNA damage response can cause apoptosis in neuronal progenitor cells during embryonic neurogenesis (reviewed in McKinnon, 2009). Mutations in DNA damage response genes can cause genomic instability disorder, with some of them characterized by microcephaly. Examples are Nijmegen 
Breakage Syndrome, caused by NBS1 gene mutations, ATR-Seckel syndrome, caused by mutations in ATR, Ataxia telangiectasia-like disorder, caused by MRE11 mutations (McKinnon, 2009). It has been shown using knockout mouse models that microcephaly observed in genomic stability disorders is caused by proliferation arrest and apoptosis of neuronal progenitor cells in response to unrepaired DNA damages (McKinnon, 2009).

Indeed, MCPH1 has been reported to have functions in the DNA damage response. MCPH1 seems to be required for the recruitment of DNA damage signaling factors involved in both main pathways ATM-dependent and ATR-dependent DNA damage response (Rai et al., 2006; Wood et al., 2008; Wood et al., 2007; Wu et al., 2009). Therefore it is possible that the reduced brain size of patients with Microcephalin mutations is caused by defective DNA damage response. However, we found that $M c p h 1^{\Delta / \Delta}$ neuronal progenitor cells showed a normal proliferation and have a normal DNA damage response (Sukchev, 2010 ; Z.W. Zhou, data not shown). In addition, we could not detect chromosomal aberrations in neuronal progenitor cells or the accumulation of unrepaired DNA breaks in the embryonic brains. The reasons for the discrepancy to the literature data are not known, but may be explained by two possibilities.

First, MCPH1 has distinct functions depending on the cell type. The role of MCPH1 in the DNA damage response in human cell lines might be more important than in mouse neuronal progenitor cells. Fibroblasts isolated from another Mcph1 knockout mouse (Mcph $\left.1^{\text {gt/gt}}\right)$ have no defects in cell cycle arrest and recruitment of DNA repair proteins after DNA damage. In addition siRNA-mediated knockdown of Mcph1 in mouse fibroblasts confirmed these observations (Trimborn et al., 2010). These data further support the notion that in mouse cells, $\mathrm{MCPH} 1$ has dispensable functions in the DNA damage response.

Second, the differences may lie also in the gene disruption strategy to generate Mcph1 knockout mice. Deletion of exons 4 and 5 presumably leads to a disruption of the first $\mathrm{N}$ terminal BRCT domain. Since the N-terminal BRCT domain of MCPH1 was shown to regulate centrosomal and cell cycle regulatory functions (Jeffers et al., 2008), we assume that these pathways are non functional in the $M c p h 1^{\Delta / \Delta}$ mice. However, we cannot rule out the possibility of the production of a truncated $\mathrm{MCPH} 1$ isoform that may contain partial functional $\mathrm{C}$-terminal BRCT domains. The C-terminal BRCT domains of MCPH1 were shown to play a role in the DNA damage response (Jeffers et al., 2008; Rai et al., 2006; Wood et al., 2008; Wood et al., 2007; Wu et al., 2009). 
Apoptosis in $M c p h 1^{\Delta / \Delta}$ neuronal progenitor cells may be triggered by mitotic failure rather than by unrepaired DNA damage. We observed mitotic spindle defects of $M c p h 1^{\Delta / \Delta}$ neuronal progenitor cells that may trigger, in severe cases, apoptotic pathways, a process termed mitotic catastrophe. Mitotic catastrophe is a programmed cell death caused by mitotic failure (Castedo et al., 2004). Consistently, increased apoptosis was specifically found in the proliferative areas VZ/SVZ of $M c p h 1^{\Delta / \Delta}$ E13.5 and E15.5 embryos, whereas no elevated apoptosis was detected in postmitotic regions of the embryonic brains. We therefore conclude that the spindle misalignment of $M c p h 1^{\Delta / \Delta}$ neuronal progenitor cells is the cause for apoptosis, rather than other defects such as altered DNA damage response.

Furthermore, overexpression of a MCPH1-GFP fusion protein in cortical neuronal progenitor cells leads to massive cell death, suggesting a dosage dependent toxic effect of MCPH1 protein level (data not shown). Interestingly, life cell imaging of MCPH1-GFP transfected neuronal progenitor cells revealed that these cells are not able to undergo mitosis (data not shown). These observations may support the suggested role of MCPH1 in the control of mitotic events.

In brains of newborn $M c p h 1^{\Delta / \Delta}$ mice we did not find increased apoptosis in any of the brain regions. However, this seems surprising as proliferation continues postnatally, such as the cell divisions of astrocytic progenitor cells. Moreover, the cerebellum develops mainly postnatally during the first three postnatal weeks in mice due to massive proliferation (Goldowitz and Hamre, 1998). Mcph1-deletion does not cause apoptosis in the developing cerebellum or in astrocytic progenitor cells. The reason for this might be a more important role of MCPH1 in the mitotic control of cortical neuronal progenitor cells during neurogenesis.

We found that MCPH1 regulates the centrosomal localization of Chk1 in G2-phase of cortical neuronal progenitor cells to prevent mitotic entry. However, we showed that MCPH1 is not absolutely required for Chk1 centrosomal localization. This is in contrast to another study that was published during the analysis of the current study, showing that lymphoblastoid cell lines isolated from MCPH1 patients show a loss of centrosomal Chk1 in about $80 \%$ of interphase cells (Tibelius et al., 2009). The discrepancy is not known and might be due to differences in the analyzed cell types. We assume that in neuronal progenitor cells, MCPH1 serves as a cofactor to regulate Chk1 centrosomal localization but is not absolutely required. We hypothesize, that complete loss of Chk1 from the centrosomes might lead to cell death rather than relatively mild mitotic spindle defects. This hypothesis is supported by our observation 
that knockdown of Chk1 in MEFs leads to dramatic increase o cells with severe mitotic failure (about 92\%), presumably leading to apoptosis by mitotic catastrophe. Consistently, another study showed that deletion of Chk1 in mouse embryonic stem cells caused massive mitotic catastrophe due to premature mitosis (Niida et al., 2005). It was also shown that Chk1 is essential for mammalian development and viability in mice and cells (Liu et al., 2000; Takai et al., 2000). The essential role of Chk1 might be restricted to its function in controlling mitotic entry rather than its function in the DNA damage response.

\subsection{Conclusions}

We identified the mechanism of $\mathrm{MCPH} 1$ in ensuring the neuron production during neurogenesis that explains the small brain size of $M c p h 1^{\Delta / \Delta}$ mice and assume a similar mechanism leading to MCPH1 in humans when defective. We conclude that the role of $\mathrm{MCPH} 1$ in the DNA damage response is dispensable for embryonic neurogenesis. We hypothesize that the chromosome condensation defects and reduced brain size are caused by distinct defective functions of $\mathrm{MCPH} 1$ in the $M c p h 1^{\Delta / \Delta}$ mice. This hypothesis is supported by the observation that cells from two other Mcph1 knockout mice show defective chromosome condensation but no microcephaly phenotype (Liang et al., 2010; Trimborn et al., 2010). The function of $\mathrm{MCPH} 1$ in chromosome condensation is presumably achieved through interaction with condensin II which is mediated by a middle domain of MCPH1 (Wood et al., 2008). The centrosomal localization of MCPH1 and presumably its role in the Chk1-Cdc25B-pathway is controlled by its N-terminal BRCT domain (Jeffers et al., 2008).

The role of MCPH1 in the centrosomal Chk1-Cdc25B-pathway is important to couple mitotic entry with the centrosome maturation (Figure 37). We found that MCPH1 contributes to the centrosomal localization of Chk1 in G2-phase cells. Centrosomal Chk1 inhibits the phosphatase Cdc25B through phosphorylation in interphase cells. This keeps Cdk1, the substrate of Cdc25B, in its phosphorylated inactive state (Figure 37A). At the onset of mitosis, Chk1 is removed from the centrosomes leading to activation of Cdc25B which subsequently 
de-phosphorylates and activates centrosomal Cdk1. The timing of Chk1 removal and Cdk1 activation is important to couple mitotic progression with the centrosome maturation. In Mcph1-deficient cells, Chk1 is lost from the centrosomes in G2-phase leading to a deregulation of Cdc25B and a premature activation of Cdk1 (Figure 37B). This leads to an uncoupling of the mitotic entry with the centrosome maturation. Immature centrosomes have a reduced ability for the microtubule nucleation and spindle organization (Thornton and Woods, 2009). Apoptosis in $M c p h 1^{\Delta / \Delta}$ neuronal progenitor cells may be triggered by mitotic failure (Figure 37B). During embryonic neurogenesis, the mitotic spindle alignment has to be controlled with high accuracy to ensure the balance between symmetric and asymmetric neuronal progenitor cell divisions. Mitotic neuronal progenitor cells with immature centrosomes may not be able to position the mitotic spindle symmetrically (Thornton and Woods, 2009). This leads to bypass of the apical components and consequently asymmetric cell division (Figure 37B). Increased unscheduled asymmetric cell divisions of Mcph1-deficient neuronal progenitor cells and additionally the high rate of apoptosis lead to a reduction of the neuronal progenitor pool and hence an insufficient production of neurons. 
A

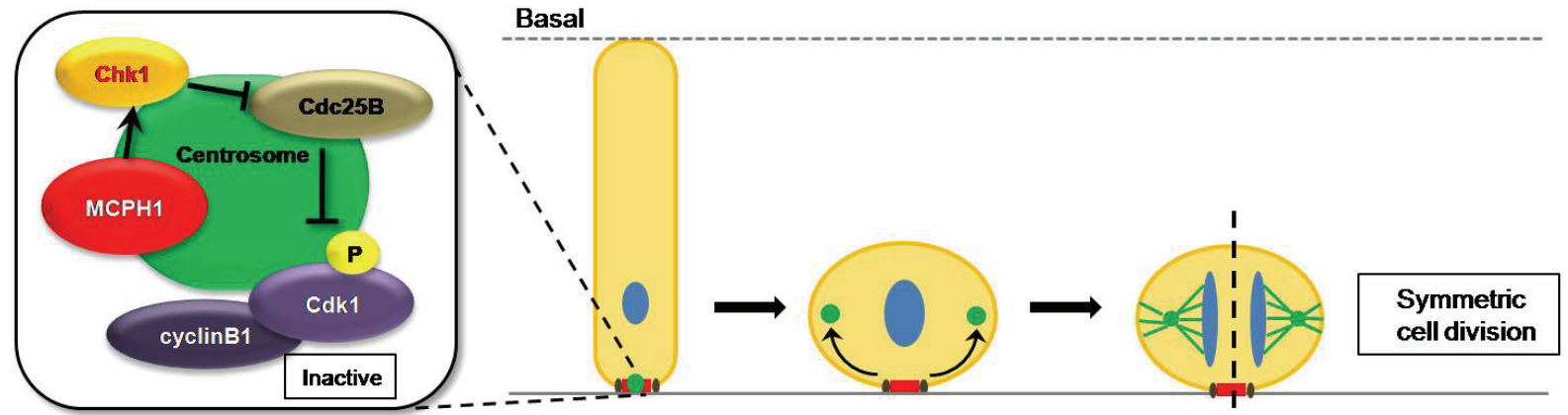

Apical

B

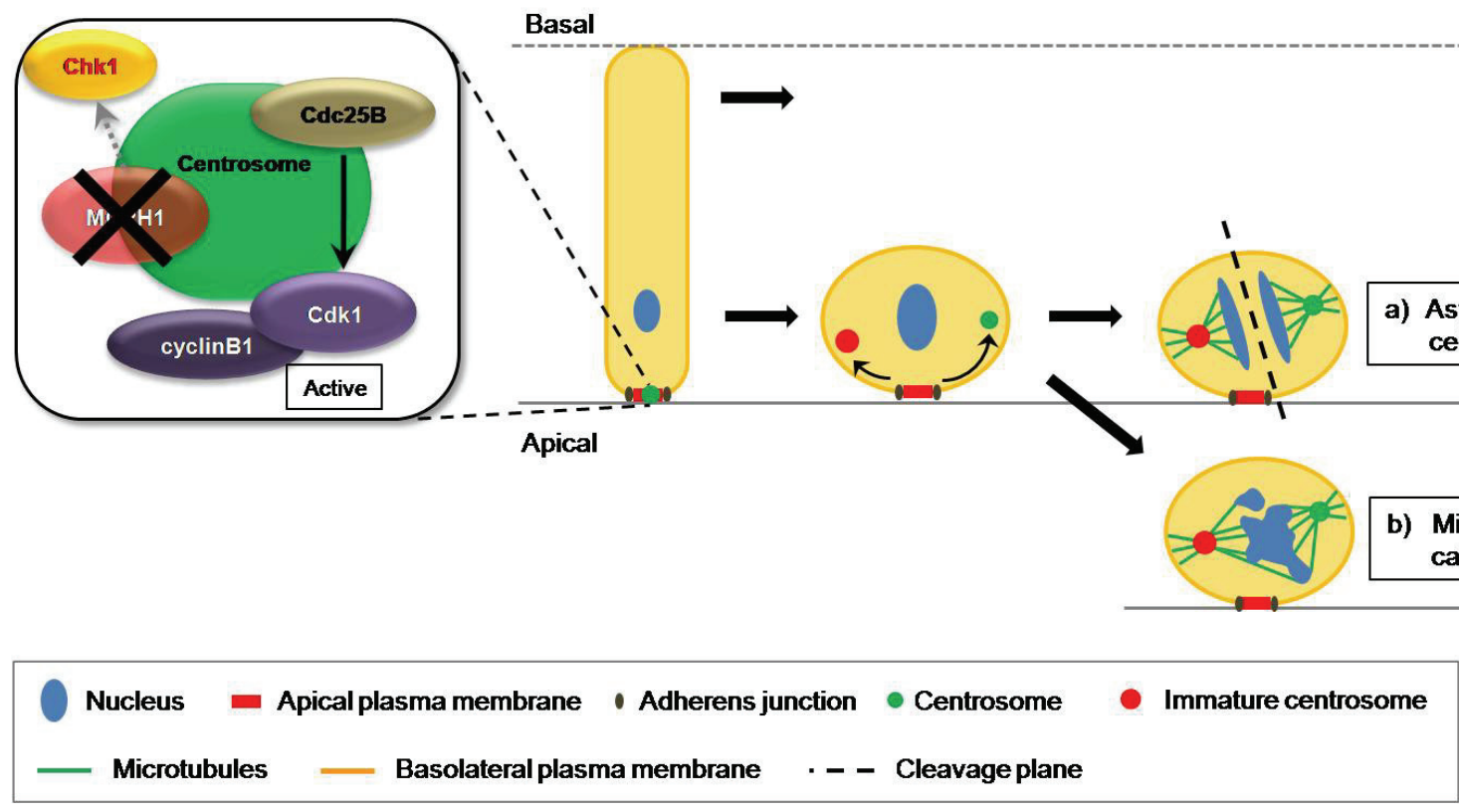

Figure 37: MCPH1 functions in the centrosomal Chk1-Cdc25B-pathway to ensure mitotic spindle alignment with high accuracy.

(A) In neuronal progenitor cells (RGCs), centrosomes are located at the apical plasma membrane during interphase. Centrosomal Chk1 inhibits Cdc25B and Cdk1 in interphase to prevent mitotic entry. $\mathrm{MCPH} 1$ contributes to Chk1 centrosomal localization in interphase RGCs. This ensures a high accuracy of mitotic spindle alignment which is important for the critical balance between symmetric and asymmetric cell divisions of neuronal progenitor cells (see also Figure 5).

(B) In Mcph1-deficient neuronal progenitor cells, a high number of cells show Chk1 loss from the centrosomes in G2-phase leading to premature activation of Cdc25B and Cdk1. This causes premature mitotic entry with an uncoupling of the mitotic progression from the centrosome cycle. Mitotic spindle poles with immature centrosomes cause either inappropriate asymmetric cell division through bypass of the apical plasma membrane and adherens junctions (a) or cell death by mitotic catastrophe (b). Both events results in reduction of the neuronal progenitor cell pool and insufficient neuron production. 


\section{References}

Aaku-Saraste, E., Hellwig, A., and Huttner, W.B. (1996). Loss of occludin and functional tight junctions, but not ZO-1, during neural tube closure--remodeling of the neuroepithelium prior to neurogenesis. Dev Biol 180, 664-679.

Alderton, G.K., Galbiati, L., Griffith, E., Surinya, K.H., Neitzel, H., Jackson, A.P., Jeggo, P.A., and O'Driscoll, M. (2006). Regulation of mitotic entry by microcephalin and its overlap with ATR signalling. Nat Cell Biol 8, 725-733.

Andersen, J.S., Wilkinson, C.J., Mayor, T., Mortensen, P., Nigg, E.A., and Mann, M. (2003). Proteomic characterization of the human centrosome by protein correlation profiling. Nature 426, 570-574.

Andersen, S.S. (1999). Molecular characteristics of the centrosome. Int Rev Cytol 187, 51109.

Anderson, S.A., Eisenstat, D.D., Shi, L., and Rubenstein, J.L. (1997). Interneuron migration from basal forebrain to neocortex: dependence on Dlx genes. Science 278, 474-476.

Angevine, J.B., Jr., and Sidman, R.L. (1961). Autoradiographic study of cell migration during histogenesis of cerebral cortex in the mouse. Nature 192, 766-768.

Anthony, T.E., Mason, H.A., Gridley, T., Fishell, G., and Heintz, N. (2005). Brain lipidbinding protein is a direct target of Notch signaling in radial glial cells. Genes Dev 19, 10281033.

Aplan, P.D., Lombardi, D.P., Ginsberg, A.M., Cossman, J., Bertness, V.L., and Kirsch, I.R. (1990). Disruption of the human SCL locus by "illegitimate" V-(D)-J recombinase activity. Science 250, 1426-1429.

Aplan, P.D., Lombardi, D.P., Reaman, G.H., Sather, H.N., Hammond, G.D., and Kirsch, I.R. (1992). Involvement of the putative hematopoietic transcription factor SCL in T-cell acute lymphoblastic leukemia. Blood 79, 1327-1333.

Bailly, E., Doree, M., Nurse, P., and Bornens, M. (1989). p34cdc2 is located in both nucleus and cytoplasm; part is centrosomally associated at G2/M and enters vesicles at anaphase. EMBO J 8, 3985-3995. 
Bailly, E., Pines, J., Hunter, T., and Bornens, M. (1992). Cytoplasmic accumulation of cyclin B1 in human cells: association with a detergent-resistant compartment and with the centrosome. J Cell Sci 101 ( Pt 3), 529-545.

Barnes, C.A. (1979). Memory deficits associated with senescence: a neurophysiological and behavioral study in the rat. J Comp Physiol Psychol 93, 74-104.

Barrera, J.A., Kao, L.R., Hammer, R.E., Seemann, J., Fuchs, J.L., and Megraw, T.L. (2010). CDK5RAP2 regulates centriole engagement and cohesion in mice. Dev Cell 18, 913926.

Basto, R., Lau, J., Vinogradova, T., Gardiol, A., Woods, C.G., Khodjakov, A., and Raff, J.W. (2006). Flies without centrioles. Cell 125, 1375-1386.

Berdnik, D., Torok, T., Gonzalez-Gaitan, M., and Knoblich, J.A. (2002). The endocytic protein alpha-Adaptin is required for numb-mediated asymmetric cell division in Drosophila. Dev Cell 3, 221-231.

Betschinger, J., and Knoblich, J.A. (2004). Dare to be different: asymmetric cell division in Drosophila, C. elegans and vertebrates. Curr Biol 14, R674-685.

Blachon, S., Gopalakrishnan, J., Omori, Y., Polyanovsky, A., Church, A., Nicastro, D., Malicki, J., and Avidor-Reiss, T. (2008). Drosophila asterless and vertebrate Cep152 Are orthologs essential for centriole duplication. Genetics 180, 2081-2094.

Boncinelli, E., Gulisano, M., and Broccoli, V. (1993). Emx and Otx homeobox genes in the developing mouse brain. J Neurobiol 24, 1356-1366.

Bond, J., Roberts, E., Mochida, G.H., Hampshire, D.J., Scott, S., Askham, J.M., Springell, K., Mahadevan, M., Crow, Y.J., Markham, A.F., et al. (2002). ASPM is a major determinant of cerebral cortical size. Nat Genet 32, 316-320.

Bond, J., Roberts, E., Springell, K., Lizarraga, S.B., Scott, S., Higgins, J., Hampshire, D.J., Morrison, E.E., Leal, G.F., Silva, E.O., et al. (2005). A centrosomal mechanism involving CDK5RAP2 and CENPJ controls brain size. Nat Genet 37, 353-355.

Booher, R.N., Holman, P.S., and Fattaey, A. (1997). Human Myt1 is a cell cycle-regulated kinase that inhibits Cdc2 but not Cdk2 activity. J Biol Chem 272, 22300-22306.

Boulder-Commitee (1970). Embryonic vertebrate central nervous system: revised terminology. The Boulder Committee. Anat Rec 166, 257-261. 
Bowman, S.K., Neumuller, R.A., Novatchkova, M., Du, Q., and Knoblich, J.A. (2006). The Drosophila NuMA Homolog Mud regulates spindle orientation in asymmetric cell division. Dev Cell 10, 731-742.

Brandeis, M., Rosewell, I., Carrington, M., Crompton, T., Jacobs, M.A., Kirk, J., Gannon, J., and Hunt, T. (1998). Cyclin B2-null mice develop normally and are fertile whereas cyclin B1-null mice die in utero. Proc Natl Acad Sci U S A 95, 4344-4349.

Brown, L., Cheng, J.T., Chen, Q., Siciliano, M.J., Crist, W., Buchanan, G., and Baer, R. (1990). Site-specific recombination of the tal-1 gene is a common occurrence in human T cell leukemia. EMBO J 9, 3343-3351.

Brunk, K., Vernay, B., Griffith, E., Reynolds, N.L., Strutt, D., Ingham, P.W., and Jackson, A.P. (2007). Microcephalin coordinates mitosis in the syncytial Drosophila embryo. J Cell Sci 120, 3578-3588.

Buchman, J.J., Tseng, H.C., Zhou, Y., Frank, C.L., Xie, Z., and Tsai, L.H. (2010). Cdk5rap2 interacts with pericentrin to maintain the neural progenitor pool in the developing neocortex. Neuron 66, 386-402.

Busch, C., Barton, O., Morgenstern, E., Gotz, C., Gunther, J., Noll, A., and Montenarh, M. (2007). The G2/M checkpoint phosphatase cdc25C is located within centrosomes. Int $\mathrm{J}$ Biochem Cell Biol 39, 1707-1713.

Calegari, F., Haubensak, W., Haffner, C., and Huttner, W.B. (2005). Selective lengthening of the cell cycle in the neurogenic subpopulation of neural progenitor cells during mouse brain development. J Neurosci 25, 6533-6538.

Calegari, F., and Huttner, W.B. (2003). An inhibition of cyclin-dependent kinases that lengthens, but does not arrest, neuroepithelial cell cycle induces premature neurogenesis. J Cell Sci 116, 4947-4955.

Campaner, S., Kaldis, P., Izraeli, S., and Kirsch, I.R. (2005). Sil phosphorylation in a Pin1 binding domain affects the duration of the spindle checkpoint. Mol Cell Biol 25, 6660-6672.

Campbell, K., and Gotz, M. (2002). Radial glia: multi-purpose cells for vertebrate brain development. Trends Neurosci 25, 235-238.

Casal, J., Gonzalez, C., Wandosell, F., Avila, J., and Ripoll, P. (1990). Abnormal meiotic spindles cause a cascade of defects during spermatogenesis in asp males of Drosophila. Development 108, 251-260. 
Castedo, M., Perfettini, J.L., Roumier, T., Andreau, K., Medema, R., and Kroemer, G. (2004). Cell death by mitotic catastrophe: a molecular definition. Oncogene 23, 2825-2837.

Cazales, M., Schmitt, E., Montembault, E., Dozier, C., Prigent, C., and Ducommun, B. (2005). CDC25B phosphorylation by Aurora-A occurs at the G2/M transition and is inhibited by DNA damage. Cell Cycle 4, 1233-1238.

Chang, P., and Stearns, T. (2000). Delta-tubulin and epsilon-tubulin: two new human centrosomal tubulins reveal new aspects of centrosome structure and function. Nat Cell Biol 2, 30-35.

Cheeseman, I.M., and Desai, A. (2008). Molecular architecture of the kinetochoremicrotubule interface. Nat Rev Mol Cell Biol 9, 33-46.

Chen, C.Y., Olayioye, M.A., Lindeman, G.J., and Tang, T.K. (2006). CPAP interacts with 143-3 in a cell cycle-dependent manner. Biochem Biophys Res Commun 342, 1203-1210.

Chenn, A., Zhang, Y.A., Chang, B.T., and McConnell, S.K. (1998). Intrinsic polarity of mammalian neuroepithelial cells. Mol Cell Neurosci 11, 183-193.

Ching, Y.P., Qi, Z., and Wang, J.H. (2000). Cloning of three novel neuronal Cdk5 activator binding proteins. Gene 242, 285-294.

Cho, J.H., Chang, C.J., Chen, C.Y., and Tang, T.K. (2006). Depletion of CPAP by RNAi disrupts centrosome integrity and induces multipolar spindles. Biochem Biophys Res Commun 339, 742-747.

Chretien, D., Buendia, B., Fuller, S.D., and Karsenti, E. (1997). Reconstruction of the centrosome cycle from cryoelectron micrographs. J Struct Biol 120, 117-133.

Collazo-Garcia, N., Scherer, P., and Aplan, P.D. (1995). Cloning and characterization of a murine SIL gene. Genomics 30, 506-513.

Corbin, J.G., Rutlin, M., Gaiano, N., and Fishell, G. (2003). Combinatorial function of the homeodomain proteins Nkx2.1 and Gsh2 in ventral telencephalic patterning. Development 130, 4895-4906.

Cox, J., Jackson, A.P., Bond, J., and Woods, C.G. (2006). What primary microcephaly can tell us about brain growth. Trends Mol Med 12, 358-366.

Cubelos, B., Sebastian-Serrano, A., Kim, S., Moreno-Ortiz, C., Redondo, J.M., Walsh, C.A., and Nieto, M. (2008). Cux-2 controls the proliferation of neuronal intermediate precursors of the cortical subventricular zone. Cereb Cortex 18, 1758-1770. 
Dai, Y., and Grant, S. (2010). New insights into checkpoint kinase 1 in the DNA damage response signaling network. Clin Cancer Res 16, 376-383.

Dammermann, A., Maddox, P.S., Desai, A., and Oegema, K. (2008). SAS-4 is recruited to a dynamic structure in newly forming centrioles that is stabilized by the gamma-tubulin-mediated addition of centriolar microtubules. J Cell Biol 180, 771-785.

De Pietri Tonelli, D., Pulvers, J.N., Haffner, C., Murchison, E.P., Hannon, G.J., and Huttner, W.B. (2008). miRNAs are essential for survival and differentiation of newborn neurons but not for expansion of neural progenitors during early neurogenesis in the mouse embryonic neocortex. Development 135, 3911-3921.

Dehay, C., and Kennedy, H. (2007). Cell-cycle control and cortical development. Nat Rev Neurosci 8, 438-450.

Dhariwala, F.A., and Rajadhyaksha, M.S. (2008). An unusual member of the Cdk family: Cdk5. Cell Mol Neurobiol 28, 351-369.

Doxsey, S. (2001). Re-evaluating centrosome function. Nat Rev Mol Cell Biol 2, 688-698.

Doxsey, S.J., Stein, P., Evans, L., Calarco, P.D., and Kirschner, M. (1994). Pericentrin, a highly conserved centrosome protein involved in microtubule organization. Cell 76, 639-650.

Du, Q., and Macara, I.G. (2004). Mammalian Pins is a conformational switch that links NuMA to heterotrimeric G proteins. Cell 119, 503-516.

Du, Q., Stukenberg, P.T., and Macara, I.G. (2001). A mammalian Partner of inscuteable binds NuMA and regulates mitotic spindle organization. Nat Cell Biol 3, 1069-1075.

Dunham, N.W., and Miya, T.S. (1957). A note on a simple apparatus for detecting neurological deficit in rats and mice. J Am Pharm Assoc Am Pharm Assoc (Baltim) 46, 208209.

Dutertre, S., Cazales, M., Quaranta, M., Froment, C., Trabut, V., Dozier, C., Mirey, G., Bouche, J.P., Theis-Febvre, N., Schmitt, E., et al. (2004). Phosphorylation of CDC25B by Aurora-A at the centrosome contributes to the G2-M transition. J Cell Sci 117, 2523-2531.

Elia, A.E., Cantley, L.C., and Yaffe, M.B. (2003a). Proteomic screen finds pSer/pThr-binding domain localizing Plk1 to mitotic substrates. Science 299, 1228-1231.

Elia, A.E., Rellos, P., Haire, L.F., Chao, J.W., Ivins, F.J., Hoepker, K., Mohammad, D., Cantley, L.C., Smerdon, S.J., and Yaffe, M.B. (2003b). The molecular basis for phosphodependent substrate targeting and regulation of Plks by the Polo-box domain. Cell $115,83-95$ 
Englund, C., Fink, A., Lau, C., Pham, D., Daza, R.A., Bulfone, A., Kowalczyk, T., and Hevner, R.F. (2005). Pax6, Tbr2, and Tbr1 are expressed sequentially by radial glia, intermediate progenitor cells, and postmitotic neurons in developing neocortex. J Neurosci 25, 247-251.

Erez, A., Castiel, A., Trakhtenbrot, L., Perelman, M., Rosenthal, E., Goldstein, I., Stettner, N., Harmelin, A., Eldar-Finkelman, H., Campaner, S., et al. (2007). The SIL gene is essential for mitotic entry and survival of cancer cells. Cancer Res 67, 4022-4027.

Ericson, J., Muhr, J., Placzek, M., Lints, T., Jessell, T.M., and Edlund, T. (1995). Sonic hedgehog induces the differentiation of ventral forebrain neurons: a common signal for ventral patterning within the neural tube. Cell $81,747-756$.

Evans, P.D., Anderson, J.R., Vallender, E.J., Choi, S.S., and Lahn, B.T. (2004a). Reconstructing the evolutionary history of microcephalin, a gene controlling human brain size. Hum Mol Genet 13, 1139-1145.

Evans, P.D., Anderson, J.R., Vallender, E.J., Gilbert, S.L., Malcom, C.M., Dorus, S., and Lahn, B.T. (2004b). Adaptive evolution of ASPM, a major determinant of cerebral cortical size in humans. Hum Mol Genet 13, 489-494.

Fietz, S.A., Kelava, I., Vogt, J., Wilsch-Brauninger, M., Stenzel, D., Fish, J.L., Corbeil, D., Riehn, A., Distler, W., Nitsch, R., et al. (2010). OSVZ progenitors of human and ferret neocortex are epithelial-like and expand by integrin signaling. Nat Neurosci 13, 690-699.

Fish, J.L., Dehay, C., Kennedy, H., and Huttner, W.B. (2008). Making bigger brains-the evolution of neural-progenitor-cell division. J Cell Sci 121, 2783-2793.

Fish, J.L., Kosodo, Y., Enard, W., Paabo, S., and Huttner, W.B. (2006). Aspm specifically maintains symmetric proliferative divisions of neuroepithelial cells. Proc Natl Acad Sci U S A 103, 10438-10443.

Fong, K.W., Choi, Y.K., Rattner, J.B., and Qi, R.Z. (2008). CDK5RAP2 is a pericentriolar protein that functions in centrosomal attachment of the gamma-tubulin ring complex. Mol Biol Cell 19, 115-125.

Frotscher, M. (2010). Role for Reelin in stabilizing cortical architecture. Trends Neurosci 33, 407-414.

Fry, A.M., Meraldi, P., and Nigg, E.A. (1998). A centrosomal function for the human Nek2 protein kinase, a member of the NIMA family of cell cycle regulators. EMBO J 17, 470-481. 
Fuerstenberg, S., Broadus, J., and Doe, C.Q. (1998). Asymmetry and cell fate in the Drosophila embryonic CNS. Int J Dev Biol 42, 379-383.

Fuja, T.J., Schwartz, P.H., Darcy, D., and Bryant, P.J. (2004). Asymmetric localization of LGN but not AGS3, two homologs of Drosophila pins, in dividing human neural progenitor cells. J Neurosci Res 75, 782-793.

Fung, T.K., and Poon, R.Y. (2005). A roller coaster ride with the mitotic cyclins. Semin Cell Dev Biol 16, 335-342.

Furuno, N., den Elzen, N., and Pines, J. (1999). Human cyclin A is required for mitosis until mid prophase. J Cell Biol 147, 295-306.

Gadisseux, J.F., and Evrard, P. (1985). Glial-neuronal relationship in the developing central nervous system. A histochemical-electron microscope study of radial glial cell particulate glycogen in normal and reeler mice and the human fetus. Dev Neurosci 7, 12-32.

Gaiano, N., and Fishell, G. (2002). The role of notch in promoting glial and neural stem cell fates. Annu Rev Neurosci 25, 471-490.

Gaiano, N., Nye, J.S., and Fishell, G. (2000). Radial glial identity is promoted by Notch1 signaling in the murine forebrain. Neuron 26, 395-404.

Gal, J.S., Morozov, Y.M., Ayoub, A.E., Chatterjee, M., Rakic, P., and Haydar, T.F. (2006). Molecular and morphological heterogeneity of neural precursors in the mouse neocortical proliferative zones. J Neurosci 26, 1045-1056.

Goldowitz, D., and Hamre, K. (1998). The cells and molecules that make a cerebellum. Trends Neurosci 21, 375-382.

Gotz, M., and Huttner, W.B. (2005). The cell biology of neurogenesis. Nat Rev Mol Cell Biol 6, 777-788.

Gould, E. (2007). How widespread is adult neurogenesis in mammals? Nat Rev Neurosci 8, 481-488.

Graser, S., Stierhof, Y.D., and Nigg, E.A. (2007). Cep68 and Cep215 (Cdk5rap2) are required for centrosome cohesion. J Cell Sci 120, 4321-4331.

Greene, N.D., and Copp, A.J. (2009). Development of the vertebrate central nervous system: formation of the neural tube. Prenat Diagn 29, 303-311.

Griffith, E., Walker, S., Martin, C.A., Vagnarelli, P., Stiff, T., Vernay, B., Al Sanna, N., Saggar, A., Hamel, B., Earnshaw, W.C., et al. (2008). Mutations in pericentrin cause Seckel syndrome with defective ATR-dependent DNA damage signaling. Nat Genet 40, 232-236. 
Guernsey, D.L., Jiang, H., Hussin, J., Arnold, M., Bouyakdan, K., Perry, S., BabineauSturk, T., Beis, J., Dumas, N., Evans, S.C., et al. (2010). Mutations in centrosomal protein CEP152 in primary microcephaly families linked to MCPH4. Am J Hum Genet 87, 40-51.

Gul, A., Hassan, M.J., Hussain, S., Raza, S.I., Chishti, M.S., and Ahmad, W. (2006). A novel deletion mutation in CENPJ gene in a Pakistani family with autosomal recessive primary microcephaly. J Hum Genet 51, 760-764.

Hagting, A., Jackman, M., Simpson, K., and Pines, J. (1999). Translocation of cyclin B1 to the nucleus at prophase requires a phosphorylation-dependent nuclear import signal. Curr Biol 9, 680-689.

Hartfuss, E., Galli, R., Heins, N., and Gotz, M. (2001). Characterization of CNS precursor subtypes and radial glia. Dev Biol 229, 15-30.

Haubensak, W., Attardo, A., Denk, W., and Huttner, W.B. (2004). Neurons arise in the basal neuroepithelium of the early mammalian telencephalon: a major site of neurogenesis. Proc Natl Acad Sci U S A 101, 3196-3201.

Haydar, T.F., Ang, E., Jr., and Rakic, P. (2003). Mitotic spindle rotation and mode of cell division in the developing telencephalon. Proc Natl Acad Sci U S A 100, 2890-2895.

Heald, R., McLoughlin, M., and McKeon, F. (1993). Human wee1 maintains mitotic timing by protecting the nucleus from cytoplasmically activated Cdc2 kinase. Cell 74, 463-474.

Heald, R., Tournebize, R., Habermann, A., Karsenti, E., and Hyman, A. (1997). Spindle assembly in Xenopus egg extracts: respective roles of centrosomes and microtubule selforganization. J Cell Biol 138, 615-628.

Hertwig, P. (1942). Neue Mutationen und Kopplungsgruppen bei der Hausmaus. Z Indukt Abstammungs Vererbungsl 80, 220-246.

Hinchcliffe, E.H., Miller, F.J., Cham, M., Khodjakov, A., and Sluder, G. (2001). Requirement of a centrosomal activity for cell cycle progression through $\mathrm{G} 1$ into $\mathrm{S}$ phase. Science 291, 1547-1550.

Hung, L.Y., Chen, H.L., Chang, C.W., Li, B.R., and Tang, T.K. (2004). Identification of a novel microtubule-destabilizing motif in CPAP that binds to tubulin heterodimers and inhibits microtubule assembly. Mol Biol Cell 15, 2697-2706.

Hung, L.Y., Tang, C.J., and Tang, T.K. (2000). Protein 4.1 R-135 interacts with a novel centrosomal protein (CPAP) which is associated with the gamma-tubulin complex. Mol Cell Biol 20, 7813-7825. 
Hutchins, J.R., Toyoda, Y., Hegemann, B., Poser, I., Heriche, J.K., Sykora, M.M., Augsburg, M., Hudecz, O., Buschhorn, B.A., Bulkescher, J., et al. (2010). Systematic analysis of human protein complexes identifies chromosome segregation proteins. Science 328, 593-599.

lacopetti, P., Michelini, M., Stuckmann, I., Oback, B., Aaku-Saraste, E., and Huttner, W.B. (1999). Expression of the antiproliferative gene TIS21 at the onset of neurogenesis identifies single neuroepithelial cells that switch from proliferative to neuron-generating division. Proc Natl Acad Sci U S A 96, 4639-4644.

Ishikawa, H., Kubo, A., and Tsukita, S. (2005). Odf2-deficient mother centrioles lack distal/subdistal appendages and the ability to generate primary cilia. Nat Cell Biol 7, 517-524.

Izraeli, S., Colaizzo-Anas, T., Bertness, V.L., Mani, K., Aplan, P.D., and Kirsch, I.R. (1997). Expression of the SIL gene is correlated with growth induction and cellular proliferation. Cell Growth Differ 8, 1171-1179.

Izraeli, S., Lowe, L.A., Bertness, V.L., Campaner, S., Hahn, H., Kirsch, I.R., and Kuehn, M.R. (2001). Genetic evidence that Sil is required for the Sonic Hedgehog response pathway. Genesis 31, 72-77.

Izraeli, S., Lowe, L.A., Bertness, V.L., Good, D.J., Dorward, D.W., Kirsch, I.R., and Kuehn, M.R. (1999). The SIL gene is required for mouse embryonic axial development and left-right specification. Nature 399, 691-694.

Izumi, Y., Ohta, N., Hisata, K., Raabe, T., and Matsuzaki, F. (2006). Drosophila Pins-binding protein Mud regulates spindle-polarity coupling and centrosome organization. Nat Cell Biol 8, 586-593.

Jackman, M., Firth, M., and Pines, J. (1995). Human cyclins B1 and B2 are localized to strikingly different structures: B1 to microtubules, B2 primarily to the Golgi apparatus. EMBO J $14,1646-1654$.

Jackman, M., Lindon, C., Nigg, E.A., and Pines, J. (2003). Active cyclin B1-Cdk1 first appears on centrosomes in prophase. Nat Cell Biol 5, 143-148.

Jackson, A.P., Eastwood, H., Bell, S.M., Adu, J., Toomes, C., Carr, I.M., Roberts, E., Hampshire, D.J., Crow, Y.J., Mighell, A.J., et al. (2002). Identification of microcephalin, a protein implicated in determining the size of the human brain. Am J Hum Genet 71, 136-142. 
Jackson, A.P., McHale, D.P., Campbell, D.A., Jafri, H., Rashid, Y., Mannan, J., Karbani, G., Corry, P., Levene, M.I., Mueller, R.F., et al. (1998). Primary autosomal recessive microcephaly (MCPH1) maps to chromosome 8p22-pter. Am J Hum Genet 63, 541-546.

Jeffers, L.J., Coull, B.J., Stack, S.J., and Morrison, C.G. (2008). Distinct BRCT domains in Mcph1/Brit1 mediate ionizing radiation-induced focus formation and centrosomal localization. Oncogene 27, 139-144.

Jin, P., Hardy, S., and Morgan, D.O. (1998). Nuclear localization of cyclin B1 controls mitotic entry after DNA damage. J Cell Biol 141, 875-885.

Joberty, G., Petersen, C., Gao, L., and Macara, I.G. (2000). The cell-polarity protein Par6 links Par3 and atypical protein kinase C to Cdc42. Nat Cell Biol 2, 531-539.

Kaindl, A.M., Passemard, S., Kumar, P., Kraemer, N., Issa, L., Zwirner, A., Gerard, B., Verloes, A., Mani, S., and Gressens, P. (2010). Many roads lead to primary autosomal recessive microcephaly. Prog Neurobiol 90, 363-383.

Kalay, E., Yigit, G., Aslan, Y., Brown, K.E., Pohl, E., Bicknell, L.S., Kayserili, H., Li, Y., Tuysuz, B., Nurnberg, G., et al. (2011). CEP152 is a genome maintenance protein disrupted in Seckel syndrome. Nat Genet 43, 23-26.

Kalsbeek, A., Bruinstroop, E., Yi, C.X., Klieverik, L.P., La Fleur, S.E., and Fliers, E. (2010). Hypothalamic control of energy metabolism via the autonomic nervous system. Ann $\mathrm{N}$ Y Acad Sci 1212, 114-129.

Kandel, E.R., Schwartz, J.H., and Jessel, T.M. (2000). Principles of Neural Science, 4 edn. Karkera, J.D., Izraeli, S., Roessler, E., Dutra, A., Kirsch, I., and Muenke, M. (2002). The genomic structure, chromosomal localization, and analysis of SIL as a candidate gene for holoprosencephaly. Cytogenet Genome Res 97, 62-67.

Keating, T.J., and Borisy, G.G. (2000). Immunostructural evidence for the template mechanism of microtubule nucleation. Nat Cell Biol 2, 352-357.

Kerzendorfer, C., and O'Driscoll, M. (2009). Human DNA damage response and repair deficiency syndromes: linking genomic instability and cell cycle checkpoint proficiency. DNA Repair (Amst) 8, 1139-1152.

Kessaris, N., Fogarty, M., lannarelli, P., Grist, M., Wegner, M., and Richardson, W.D. (2006). Competing waves of oligodendrocytes in the forebrain and postnatal elimination of an embryonic lineage. Nat Neurosci 9, 173-179. 
Khodjakov, A., Cole, R.W., Oakley, B.R., and Rieder, C.L. (2000). Centrosome-independent mitotic spindle formation in vertebrates. Curr Biol 10, 59-67.

Kirkham, M., Muller-Reichert, T., Oegema, K., Grill, S., and Hyman, A.A. (2003). SAS-4 is a C. elegans centriolar protein that controls centrosome size. Cell 112, 575-587.

Kitagawa, K., and Hieter, P. (2001). Evolutionary conservation between budding yeast and human kinetochores. Nat Rev Mol Cell Biol 2, 678-687.

Klezovitch, O., Fernandez, T.E., Tapscott, S.J., and Vasioukhin, V. (2004). Loss of cell polarity causes severe brain dysplasia in Lgl1 knockout mice. Genes Dev 18, 559-571.

Knoblich, J.A. (2008). Mechanisms of asymmetric stem cell division. Cell 132, 583-597.

Konno, D., Shioi, G., Shitamukai, A., Mori, A., Kiyonari, H., Miyata, T., and Matsuzaki, F. (2008). Neuroepithelial progenitors undergo LGN-dependent planar divisions to maintain selfrenewability during mammalian neurogenesis. Nat Cell Biol 10, 93-101.

Kosodo, Y., Roper, K., Haubensak, W., Marzesco, A.M., Corbeil, D., and Huttner, W.B. (2004). Asymmetric distribution of the apical plasma membrane during neurogenic divisions of mammalian neuroepithelial cells. EMBO J 23, 2314-2324.

Kouprina, N., Pavlicek, A., Collins, N.K., Nakano, M., Noskov, V.N., Ohzeki, J., Mochida, G.H., Risinger, J.I., Goldsmith, P., Gunsior, M., et al. (2005). The microcephaly ASPM gene is expressed in proliferating tissues and encodes for a mitotic spindle protein. Hum Mol Genet $14,2155-2165$.

Koyanagi, M., Hijikata, M., Watashi, K., Masui, O., and Shimotohno, K. (2005). Centrosomal P4.1-associated protein is a new member of transcriptional coactivators for nuclear factor-kappaB. J Biol Chem 280, 12430-12437.

Kramer, A., Mailand, N., Lukas, C., Syljuasen, R.G., Wilkinson, C.J., Nigg, E.A., Bartek, J., and Lukas, J. (2004). Centrosome-associated Chk1 prevents premature activation of cyclin-B-Cdk1 kinase. Nat Cell Biol 6, 884-891.

Kriegstein, A., and Alvarez-Buylla, A. (2009). The glial nature of embryonic and adult neural stem cells. Annu Rev Neurosci 32, 149-184.

Kumar, A., Girimaji, S.C., Duvvari, M.R., and Blanton, S.H. (2009). Mutations in STIL, encoding a pericentriolar and centrosomal protein, cause primary microcephaly. Am J Hum Genet 84, 286-290.

Kumar, A., Markandaya, M., and Girimaji, S.C. (2002). Primary microcephaly: microcephalin and ASPM determine the size of the human brain. J Biosci 27, 629-632. 
Lammer, C., Wagerer, S., Saffrich, R., Mertens, D., Ansorge, W., and Hoffmann, I. (1998). The cdc25B phosphatase is essential for the G2/M phase transition in human cells. J Cell Sci 111 (Pt 16), 2445-2453.

Landrieu, P., and Goffinet, A. (1979). Mitotic spindle fiber orientation in relation to cell migration in the neo-cortex of normal and reeler mouse. Neurosci Lett 13, 69-72.

Lange, B.M., and Gull, K. (1995). A molecular marker for centriole maturation in the mammalian cell cycle. J Cell Biol 130, 919-927.

Lange, C., Huttner, W.B., and Calegari, F. (2009). Cdk4/cyclinD1 overexpression in neural stem cells shortens G1, delays neurogenesis, and promotes the generation and expansion of basal progenitors. Cell Stem Cell 5, 320-331.

Leal, G.F., Roberts, E., Silva, E.O., Costa, S.M., Hampshire, D.J., and Woods, C.G. (2003). A novel locus for autosomal recessive primary microcephaly (MCPH6) maps to 13q12.2. J Med Genet 40, 540-542.

Leidel, S., and Gonczy, P. (2003). SAS-4 is essential for centrosome duplication in C elegans and is recruited to daughter centrioles once per cell cycle. Dev Cell 4, 431-439.

Li, H.S., Wang, D., Shen, Q., Schonemann, M.D., Gorski, J.A., Jones, K.R., Temple, S., Jan, L.Y., and Jan, Y.N. (2003). Inactivation of Numb and Numblike in embryonic dorsal forebrain impairs neurogenesis and disrupts cortical morphogenesis. Neuron 40, 1105-1118.

Li, J., Meyer, A.N., and Donoghue, D.J. (1997). Nuclear localization of cyclin B1 mediates its biological activity and is regulated by phosphorylation. Proc Natl Acad Sci U S A 94, 502-507.

Liang, Y., Gao, H., Lin, S.Y., Peng, G., Huang, X., Zhang, P., Goss, J.A., Brunicardi, F.C., Multani, A.S., Chang, S., et al. (2010). BRIT1/MCPH1 is essential for mitotic and meiotic recombination DNA repair and maintaining genomic stability in mice. PLoS Genet 6, e1000826.

Lin, D., Edwards, A.S., Fawcett, J.P., Mbamalu, G., Scott, J.D., and Pawson, T. (2000). A mammalian PAR-3-PAR-6 complex implicated in Cdc42/Rac1 and aPKC signalling and cell polarity. Nat Cell Biol 2, 540-547.

Lin, S.Y., and Elledge, S.J. (2003). Multiple tumor suppressor pathways negatively regulate telomerase. Cell 113, 881-889.

Lin, S.Y., Rai, R., Li, K., Xu, Z.X., and Elledge, S.J. (2005). BRIT1/MCPH1 is a DNA damage responsive protein that regulates the Brca1-Chk1 pathway, implicating checkpoint dysfunction in microcephaly. Proc Natl Acad Sci U S A 102, 15105-15109. 
Lindqvist, A., Kallstrom, H., Lundgren, A., Barsoum, E., and Rosenthal, C.K. (2005). Cdc25B cooperates with Cdc25A to induce mitosis but has a unique role in activating cyclin B1-Cdk1 at the centrosome. J Cell Biol 171, 35-45.

Liu, Q., Guntuku, S., Cui, X.S., Matsuoka, S., Cortez, D., Tamai, K., Luo, G., CarattiniRivera, S., DeMayo, F., Bradley, A., et al. (2000). Chk1 is an essential kinase that is regulated by Atr and required for the G(2)/M DNA damage checkpoint. Genes Dev 14, 14481459.

Lizarraga, S.B., Margossian, S.P., Harris, M.H., Campagna, D.R., Han, A.P., Blevins, S., Mudbhary, R., Barker, J.E., Walsh, C.A., and Fleming, M.D. (2010). Cdk5rap2 regulates centrosome function and chromosome segregation in neuronal progenitors. Development 137 , 1907-1917.

Loffler, H., Rebacz, B., Ho, A.D., Lukas, J., Bartek, J., and Kramer, A. (2006). Chk1dependent regulation of Cdc25B functions to coordinate mitotic events. Cell Cycle 5, 25432547.

Lucas, E.P., and Raff, J.W. (2007). Maintaining the proper connection between the centrioles and the pericentriolar matrix requires Drosophila centrosomin. J Cell Biol 178, 725-732.

Malumbres, M., and Barbacid, M. (2009). Cell cycle, CDKs and cancer: a changing paradigm. Nat Rev Cancer 9, 153-166.

Manke, I.A., Lowery, D.M., Nguyen, A., and Yaffe, M.B. (2003). BRCT repeats as phosphopeptide-binding modules involved in protein targeting. Science 302, 636-639.

Marthiens, V., and ffrench-Constant, C. (2009). Adherens junction domains are split by asymmetric division of embryonic neural stem cells. EMBO Rep 10, 515-520.

Marthiens, V., Kazanis, I., Moss, L., Long, K., and Ffrench-Constant, C. (2010). Adhesion molecules in the stem cell niche--more than just staying in shape? J Cell Sci 123, 1613-1622.

McKinnon, P.J. (2009). DNA repair deficiency and neurological disease. Nat Rev Neurosci 10, 100-112.

Mission, J.P., Takahashi, T., and Caviness, V.S., Jr. (1991). Ontogeny of radial and other astroglial cells in murine cerebral cortex. Glia 4, 138-148.

Miyata, T., Kawaguchi, A., Saito, K., Kawano, M., Muto, T., and Ogawa, M. (2004). Asymmetric production of surface-dividing and non-surface-dividing cortical progenitor cells. Development 131, 3133-3145. 
Mogensen, M.M., Malik, A., Piel, M., Bouckson-Castaing, V., and Bornens, M. (2000). Microtubule minus-end anchorage at centrosomal and non-centrosomal sites: the role of ninein. J Cell Sci 113 ( Pt 17), 3013-3023.

Moore, J.D., Yang, J., Truant, R., and Kornbluth, S. (1999). Nuclear import of Cdk/cyclin complexes: identification of distinct mechanisms for import of Cdk2/cyclin E and Cdc2/cyclin B1. J Cell Biol 144, 213-224.

Morgan, D.O., ed. (2007). The cell cycle: Principles of control (London: New Science Press Ltd).

Moritz, M., Braunfeld, M.B., Guenebaut, V., Heuser, J., and Agard, D.A. (2000). Structure of the gamma-tubulin ring complex: a template for microtubule nucleation. Nat Cell Biol 2, 365370.

Moynihan, L., Jackson, A.P., Roberts, E., Karbani, G., Lewis, I., Corry, P., Turner, G., Mueller, R.F., Lench, N.J., and Woods, C.G. (2000). A third novel locus for primary autosomal recessive microcephaly maps to chromosome 9q34. Am J Hum Genet 66, 724-727. Musacchio, A., and Salmon, E.D. (2007). The spindle-assembly checkpoint in space and time. Nat Rev Mol Cell Biol 8, 379-393.

Nasmyth, K., Peters, J.M., and UhImann, F. (2000). Splitting the chromosome: cutting the ties that bind sister chromatids. Science 288, 1379-1385.

Neitzel, H., Neumann, L.M., Schindler, D., Wirges, A., Tonnies, H., Trimborn, M., Krebsova, A., Richter, R., and Sperling, K. (2002). Premature chromosome condensation in humans associated with microcephaly and mental retardation: a novel autosomal recessive condition. Am J Hum Genet 70, 1015-1022.

Nguyen-Ngoc, T., Afshar, K., and Gonczy, P. (2007). Coupling of cortical dynein and G alpha proteins mediates spindle positioning in Caenorhabditis elegans. Nat Cell Biol 9, 12941302.

Nicholas, A.K., Khurshid, M., Desir, J., Carvalho, O.P., Cox, J.J., Thornton, G., Kausar, R., Ansar, M., Ahmad, W., Verloes, A., et al. (2010). WDR62 is associated with the spindle pole and is mutated in human microcephaly. Nat Genet 42, 1010-1014.

Nieuwenhuys, R., Voogd, J., and van Huijzen, C. (2007). The Human Central Nervous System, 4 edn. 
Niida, H., Tsuge, S., Katsuno, Y., Konishi, A., Takeda, N., and Nakanishi, M. (2005). Depletion of Chk1 leads to premature activation of Cdc2-cyclin B and mitotic catastrophe. J Biol Chem 280, 39246-39252.

Nipper, R.W., Siller, K.H., Smith, N.R., Doe, C.Q., and Prehoda, K.E. (2007). Galphai generates multiple Pins activation states to link cortical polarity and spindle orientation in Drosophila neuroblasts. Proc Natl Acad Sci U S A 104, 14306-14311.

Noctor, S.C., Flint, A.C., Weissman, T.A., Dammerman, R.S., and Kriegstein, A.R. (2001). Neurons derived from radial glial cells establish radial units in neocortex. Nature 409, 714-720. Noctor, S.C., Martinez-Cerdeno, V., Ivic, L., and Kriegstein, A.R. (2004). Cortical neurons arise in symmetric and asymmetric division zones and migrate through specific phases. Nat Neurosci 7, 136-144.

Noctor, S.C., Martinez-Cerdeno, V., and Kriegstein, A.R. (2007). Contribution of intermediate progenitor cells to cortical histogenesis. Arch Neurol 64, 639-642.

O'Farrell, P.H. (2001). Triggering the all-or-nothing switch into mitosis. Trends Cell Biol 11, 512-519.

Ohshima, T., Ward, J.M., Huh, C.G., Longenecker, G., Veeranna, Pant, H.C., Brady, R.O., Martin, L.J., and Kulkarni, A.B. (1996). Targeted disruption of the cyclin-dependent kinase 5 gene results in abnormal corticogenesis, neuronal pathology and perinatal death. Proc Natl Acad Sci U S A 93, 11173-11178.

Paoletti, A., Moudjou, M., Paintrand, M., Salisbury, J.L., and Bornens, M. (1996). Most of centrin in animal cells is not centrosome-associated and centrosomal centrin is confined to the distal lumen of centrioles. J Cell Sci 109 (Pt 13), 3089-3102.

Passemard, S., Titomanlio, L., Elmaleh, M., Afenjar, A., Alessandri, J.L., Andria, G., de Villemeur, T.B., Boespflug-Tanguy, O., Burglen, L., Del Giudice, E., et al. (2009). Expanding the clinical and neuroradiologic phenotype of primary microcephaly due to ASPM mutations. Neurology 73, 962-969.

Pattison, L., Crow, Y.J., Deeble, V.J., Jackson, A.P., Jafri, H., Rashid, Y., Roberts, E., and Woods, C.G. (2000). A fifth locus for primary autosomal recessive microcephaly maps to chromosome 1q31. Am J Hum Genet 67, 1578-1580.

Peng, G., Yim, E.K., Dai, H., Jackson, A.P., Burgt, I., Pan, M.R., Hu, R., Li, K., and Lin, S.Y. (2009). BRIT1/MCPH1 links chromatin remodelling to DNA damage response. Nat Cell Biol 11, 865-872. 
Petersen, C., Fuzesi, L., and Hoyer-Fender, S. (1999). Outer dense fibre proteins from human sperm tail: molecular cloning and expression analyses of two cDNA transcripts encoding proteins of approximately 70 kDa. Mol Hum Reprod 5, 627-635.

Petersen, P.H., Zou, K., Krauss, S., and Zhong, W. (2004). Continuing role for mouse Numb and Numbl in maintaining progenitor cells during cortical neurogenesis. Nat Neurosci 7, 803811.

Pfaff, K.L., Straub, C.T., Chiang, K., Bear, D.M., Zhou, Y., and Zon, L.I. (2007). The zebra fish cassiopeia mutant reveals that SIL is required for mitotic spindle organization. Mol Cell Biol 27, 5887-5897.

Pilaz, L.J., Patti, D., Marcy, G., Ollier, E., Pfister, S., Douglas, R.J., Betizeau, M., Gautier, E., Cortay, V., Doerflinger, N., et al. (2009). Forced G1-phase reduction alters mode of division, neuron number, and laminar phenotype in the cerebral cortex. Proc Natl Acad Sci U S A 106, 21924-21929.

Pinto, L., Drechsel, D., Schmid, M.T., Ninkovic, J., Irmler, M., Brill, M.S., Restani, L., Gianfranceschi, L., Cerri, C., Weber, S.N., et al. (2009). AP2gamma regulates basal progenitor fate in a region- and layer-specific manner in the developing cortex. Nat Neurosci 12, $1229-1237$.

Plowman, E.K., and Kleim, J.A. (2010). Motor cortex reorganization across the lifespan. J Commun Disord 43, 286-294.

Ponting, C., and Jackson, A.P. (2005). Evolution of primary microcephaly genes and the enlargement of primate brains. Curr Opin Genet Dev 15, 241-248.

Porter, L.A., Cukier, I.H., and Lee, J.M. (2003). Nuclear localization of cyclin B1 regulates DNA damage-induced apoptosis. Blood 101, 1928-1933.

Pulvers, J.N., Bryk, J., Fish, J.L., Wilsch-Brauninger, M., Arai, Y., Schreier, D., Naumann, R., Helppi, J., Habermann, B., Vogt, J., et al. (2010). Mutations in mouse Aspm (abnormal spindle-like microcephaly associated) cause not only microcephaly but also major defects in the germline. Proc Natl Acad Sci U S A 107, 16595-16600.

Qian, X., Shen, Q., Goderie, S.K., He, W., Capela, A., Davis, A.A., and Temple, S. (2000). Timing of CNS cell generation: a programmed sequence of neuron and glial cell production from isolated murine cortical stem cells. Neuron $28,69-80$. 
Rai, R., Dai, H., Multani, A.S., Li, K., Chin, K., Gray, J., Lahad, J.P., Liang, J., Mills, G.B., Meric-Bernstam, F., et al. (2006). BRIT1 regulates early DNA damage response, chromosomal integrity, and cancer. Cancer Cell 10, 145-157.

Rai, R., Phadnis, A., Haralkar, S., Badwe, R.A., Dai, H., Li, K., and Lin, S.Y. (2008). Differential regulation of centrosome integrity by DNA damage response proteins. Cell Cycle 7 , 2225-2233.

Rakic, P. (1972). Mode of cell migration to the superficial layers of fetal monkey neocortex. J Comp Neurol 145, 61-83.

Rakic, P. (1974). Neurons in rhesus monkey visual cortex: systematic relation between time of origin and eventual disposition. Science 183, 425-427.

Rakic, P. (1995). A small step for the cell, a giant leap for mankind: a hypothesis of neocortical expansion during evolution. Trends Neurosci 18, 383-388.

Rakic, P. (2009). Evolution of the neocortex: a perspective from developmental biology. Nat Rev Neurosci 10, 724-735.

Rallu, M., Machold, R., Gaiano, N., Corbin, J.G., McMahon, A.P., and Fishell, G. (2002). Dorsoventral patterning is established in the telencephalon of mutants lacking both Gli3 and Hedgehog signaling. Development 129, 4963-4974.

Rasin, M.R., Gazula, V.R., Breunig, J.J., Kwan, K.Y., Johnson, M.B., Liu-Chen, S., Li, H.S., Jan, L.Y., Jan, Y.N., Rakic, P., et al. (2007). Numb and Numbl are required for maintenance of cadherin-based adhesion and polarity of neural progenitors. Nat Neurosci 10 , 819-827.

Rauch, A., Thiel, C.T., Schindler, D., Wick, U., Crow, Y.J., Ekici, A.B., van Essen, A.J., Goecke, T.O., Al-Gazali, L., Chrzanowska, K.H., et al. (2008). Mutations in the pericentrin (PCNT) gene cause primordial dwarfism. Science 319, 816-819.

Rebollo, E., Sampaio, P., Januschke, J., Llamazares, S., Varmark, H., and Gonzalez, C. (2007). Functionally unequal centrosomes drive spindle orientation in asymmetrically dividing Drosophila neural stem cells. Dev Cell 12, 467-474.

Rickmyre, J.L., Dasgupta, S., Ooi, D.L., Keel, J., Lee, E., Kirschner, M.W., Waddell, S., and Lee, L.A. (2007). The Drosophila homolog of MCPH1, a human microcephaly gene, is required for genomic stability in the early embryo. J Cell Sci 120, 3565-3577. 
Roberts, E., Hampshire, D.J., Pattison, L., Springell, K., Jafri, H., Corry, P., Mannon, J., Rashid, Y., Crow, Y., Bond, J., et al. (2002). Autosomal recessive primary microcephaly: an analysis of locus heterogeneity and phenotypic variation. J Med Genet 39, 718-721.

Rogakou, E.P., Pilch, D.R., Orr, A.H., Ivanova, V.S., and Bonner, W.M. (1998). DNA double-stranded breaks induce histone H2AX phosphorylation on serine 139. J Biol Chem 273, 5858-5868.

Rubenstein, J.L., Shimamura, K., Martinez, S., and Puelles, L. (1998). Regionalization of the prosencephalic neural plate. Annu Rev Neurosci 21, 445-477.

Rusan, N.M., and Peifer, M. (2007). A role for a novel centrosome cycle in asymmetric cell division. J Cell Biol 177, 13-20.

Sahara, S., and O'Leary, D.D. (2009). Fgf10 regulates transition period of cortical stem cell differentiation to radial glia controlling generation of neurons and basal progenitors. Neuron 63, 48-62.

Sanada, K., and Tsai, L.H. (2005). G protein betagamma subunits and AGS3 control spindle orientation and asymmetric cell fate of cerebral cortical progenitors. Cell 122, 119-131.

Sauer, F.C. (1935). Mitosis in the neural tube. J Comp Neurol 62, 377-405.

Saunders, R.D., Avides, M.C., Howard, T., Gonzalez, C., and Glover, D.M. (1997). The Drosophila gene abnormal spindle encodes a novel microtubule-associated protein that associates with the polar regions of the mitotic spindle. J Cell Biol 137, 881-890.

Schmitt, E., Boutros, R., Froment, C., Monsarrat, B., Ducommun, B., and Dozier, C. (2006). CHK1 phosphorylates CDC25B during the cell cycle in the absence of DNA damage. J Cell Sci 119, 4269-4275.

Schober, M., Schaefer, M., and Knoblich, J.A. (1999). Bazooka recruits Inscuteable to orient asymmetric cell divisions in Drosophila neuroblasts. Nature 402, 548-551.

Seuntjens, E., Nityanandam, A., Miquelajauregui, A., Debruyn, J., Stryjewska, A., Goebbels, S., Nave, K.A., Huylebroeck, D., and Tarabykin, V. (2009). Sip1 regulates sequential fate decisions by feedback signaling from postmitotic neurons to progenitors. Nat Neurosci 12, 1373-1380.

Sharp, D.J., Rogers, G.C., and Scholey, J.M. (2000). Microtubule motors in mitosis. Nature 407, 41-47. 
Shen, J., Eyaid, W., Mochida, G.H., Al-Moayyad, F., Bodell, A., Woods, C.G., and Walsh, C.A. (2005). ASPM mutations identified in patients with primary microcephaly and seizures. J Med Genet 42, 725-729.

Shen, Q., Wang, Y., Dimos, J.T., Fasano, C.A., Phoenix, T.N., Lemischka, I.R., Ivanova, N.B., Stifani, S., Morrisey, E.E., and Temple, S. (2006). The timing of cortical neurogenesis is encoded within lineages of individual progenitor cells. Nat Neurosci 9, 743-751.

Shen, Q., Zhong, W., Jan, Y.N., and Temple, S. (2002). Asymmetric Numb distribution is critical for asymmetric cell division of mouse cerebral cortical stem cells and neuroblasts. Development 129, 4843-4853.

Shimamura, K., Martinez, S., Puelles, L., and Rubenstein, J.L. (1997). Patterns of gene expression in the neural plate and neural tube subdivide the embryonic forebrain into transverse and longitudinal domains. Dev Neurosci 19, 88-96.

Siegenthaler, J.A., Ashique, A.M., Zarbalis, K., Patterson, K.P., Hecht, J.H., Kane, M.A., Folias, A.E., Choe, Y., May, S.R., Kume, T., et al. (2009). Retinoic acid from the meninges regulates cortical neuron generation. Cell 139, 597-609.

Siller, K.H., Cabernard, C., and Doe, C.Q. (2006). The NuMA-related Mud protein binds Pins and regulates spindle orientation in Drosophila neuroblasts. Nat Cell Biol 8, 594-600.

Silver, D.L., Watkins-Chow, D.E., Schreck, K.C., Pierfelice, T.J., Larson, D.M., Burnetti, A.J., Liaw, H.J., Myung, K., Walsh, C.A., Gaiano, N., et al. (2010). The exon junction complex component Magoh controls brain size by regulating neural stem cell division. Nat Neurosci 13, 551-558.

Smart, I.H. (1973). Proliferative characteristics of the ependymal layer during the early development of the mouse neocortex: a pilot study based on recording the number, location and plane of cleavage of mitotic figures. J Anat 116, 67-91.

Smart, I.H., Dehay, C., Giroud, P., Berland, M., and Kennedy, H. (2002). Unique morphological features of the proliferative zones and postmitotic compartments of the neural epithelium giving rise to striate and extrastriate cortex in the monkey. Cereb Cortex 12, 37-53.

Smith, C.A., Dho, S.E., Donaldson, J., Tepass, U., and McGlade, C.J. (2004). The cell fate determinant numb interacts with EHD/Rme-1 family proteins and has a role in endocytic recycling. Mol Biol Cell 15, 3698-3708. 
Smith, C.A., Lau, K.M., Rahmani, Z., Dho, S.E., Brothers, G., She, Y.M., Berry, D.M., Bonneil, E., Thibault, P., Schweisguth, F., et al. (2007a). aPKC-mediated phosphorylation regulates asymmetric membrane localization of the cell fate determinant Numb. EMBO J 26, 468-480.

Smith, C.M., Finger, J.H., Hayamizu, T.F., McCright, I.J., Eppig, J.T., Kadin, J.A., Richardson, J.E., and Ringwald, M. (2007b). The mouse Gene Expression Database (GXD): 2007 update. Nucleic Acids Res 35, D618-623.

Stevens, N.R., Raposo, A.A., Basto, R., St Johnston, D., and Raff, J.W. (2007). From stem cell to embryo without centrioles. Curr Biol 17, 1498-1503.

Sukchev, M. (2010). Cellular and genetic studies of the Mcph1 gene. PhD Thesis; FriedrichSchiller-Universitaet Jena.

Suzuki, A., and Ohno, S. (2006). The PAR-aPKC system: lessons in polarity. J Cell Sci 119, 979-987.

Takahashi, T., Nowakowski, R.S., and Caviness, V.S., Jr. (1993). Cell cycle parameters and patterns of nuclear movement in the neocortical proliferative zone of the fetal mouse. $\mathrm{J}$ Neurosci 13, 820-833.

Takahashi, T., Nowakowski, R.S., and Caviness, V.S., Jr. (1995). The cell cycle of the pseudostratified ventricular epithelium of the embryonic murine cerebral wall. J Neurosci 15, 6046-6057.

Takai, H., Tominaga, K., Motoyama, N., Minamishima, Y.A., Nagahama, H., Tsukiyama, T., Ikeda, K., Nakayama, K., and Nakanishi, M. (2000). Aberrant cell cycle checkpoint function and early embryonic death in Chk1(-/-) mice. Genes Dev 14, 1439-1447.

Thornton, G.K., and Woods, C.G. (2009). Primary microcephaly: do all roads lead to Rome? Trends Genet 25, 501-510.

Tibelius, A., Marhold, J., Zentgraf, H., Heilig, C.E., Neitzel, H., Ducommun, B., Rauch, A., Ho, A.D., Bartek, J., and Kramer, A. (2009). Microcephalin and pericentrin regulate mitotic entry via centrosome-associated Chk1. J Cell Biol 185, 1149-1157.

Trimborn, M., Bell, S.M., Felix, C., Rashid, Y., Jafri, H., Griffiths, P.D., Neumann, L.M., Krebs, A., Reis, A., Sperling, K., et al. (2004). Mutations in microcephalin cause aberrant regulation of chromosome condensation. Am J Hum Genet 75, 261-266. 
Trimborn, M., Ghani, M., Walther, D.J., Dopatka, M., Dutrannoy, V., Busche, A., Meyer, F., Nowak, S., Nowak, J., Zabel, C., et al. (2010). Establishment of a mouse model with misregulated chromosome condensation due to defective Mcph1 function. PLoS One 5, e9242.

Trimborn, M., Richter, R., Sternberg, N., Gavvovidis, I., Schindler, D., Jackson, A.P., Prott, E.C., Sperling, K., Gillessen-Kaesbach, G., and Neitzel, H. (2005). The first missense alteration in the MCPH1 gene causes autosomal recessive microcephaly with an extremely mild cellular and clinical phenotype. Hum Mutat 26, 496.

Trimborn, M., Schindler, D., Neitzel, H., and Hirano, T. (2006). Misregulated chromosome condensation in MCPH1 primary microcephaly is mediated by condensin II. Cell Cycle 5, 322326.

Tronche, F., Kellendonk, C., Kretz, O., Gass, P., Anlag, K., Orban, P.C., Bock, R., Klein, R., and Schutz, G. (1999). Disruption of the glucocorticoid receptor gene in the nervous system results in reduced anxiety. Nat Genet 23, 99-103.

van der Voet, M., Berends, C.W., Perreault, A., Nguyen-Ngoc, T., Gonczy, P., Vidal, M., Boxem, M., and van den Heuvel, S. (2009). NuMA-related LIN-5, ASPM-1, calmodulin and dynein promote meiotic spindle rotation independently of cortical LIN-5/GPR/Galpha. Nat Cell Biol 11, 269-277.

van Vugt, M.A., and Medema, R.H. (2005). Getting in and out of mitosis with Polo-like kinase1. Oncogene 24, 2844-2859.

Wang, X., Ching, Y.P., Lam, W.H., Qi, Z., Zhang, M., and Wang, J.H. (2000). Identification of a common protein association region in the neuronal Cdk5 activator. J Biol Chem 275, 31763-31769.

Wang, X., Tsai, J.W., Imai, J.H., Lian, W.N., Vallee, R.B., and Shi, S.H. (2009). Asymmetric centrosome inheritance maintains neural progenitors in the neocortex. Nature 461, 947-955.

Wang, Y.Q., and Su, B. (2004). Molecular evolution of microcephalin, a gene determining human brain size. Hum Mol Genet 13, 1131-1137.

Warmerdam, D.O., and Kanaar, R. (2010). Dealing with DNA damage: relationships between checkpoint and repair pathways. Mutat Res 704, 2-11.

Wasserman, T., Katsenelson, K., Daniliuc, S., Hasin, T., Choder, M., and Aronheim, A. (2010). A novel c-Jun N-terminal kinase (JNK)-binding protein WDR62 is recruited to stress granules and mediates a nonclassical JNK activation. Mol Biol Cell 21, 117-130. 
Watanabe, N., Arai, H., Iwasaki, J., Shiina, M., Ogata, K., Hunter, T., and Osada, H. (2005). Cyclin-dependent kinase (CDK) phosphorylation destabilizes somatic Wee1 via multiple pathways. Proc Natl Acad Sci U S A 102, 11663-11668.

Williams, M.F. (2002). Primate encephalization and intelligence. Med Hypotheses 58, 284290.

Wilsker, D., Petermann, E., Helleday, T., and Bunz, F. (2008). Essential function of Chk1 can be uncoupled from DNA damage checkpoint and replication control. Proc Natl Acad Sci U S A 105, 20752-20757.

Wittmann, T., Boleti, H., Antony, C., Karsenti, E., and Vernos, I. (1998). Localization of the kinesin-like protein Xklp2 to spindle poles requires a leucine zipper, a microtubule-associated protein, and dynein. J Cell Biol 143, 673-685.

Wood, J.L., Liang, Y., Li, K., and Chen, J. (2008). Microcephalin/MCPH1 associates with the Condensin II complex to function in homologous recombination repair. J Biol Chem 283, 29586-29592.

Wood, J.L., Singh, N., Mer, G., and Chen, J. (2007). MCPH1 functions in an H2AXdependent but MDC1-independent pathway in response to DNA damage. J Biol Chem 282, 35416-35423.

Woods, C.G. (2004). Human microcephaly. Curr Opin Neurobiol 14, 112-117.

Woods, C.G., Bond, J., and Enard, W. (2005). Autosomal recessive primary microcephaly $(\mathrm{MCPH})$ : a review of clinical, molecular, and evolutionary findings. Am J Hum Genet 76, 717728.

Wu, X., Mondal, G., Wang, X., Wu, J., Yang, L., Pankratz, V.S., Rowley, M., and Couch, F.J. (2009). Microcephalin regulates BRCA2 and Rad51-associated DNA double-strand break repair. Cancer Res 69, 5531-5536.

Xu, X., Lee, J., and Stern, D.F. (2004). Microcephalin is a DNA damage response protein involved in regulation of CHK1 and BRCA1. J Biol Chem 279, 34091-34094.

Yang, J., Bardes, E.S., Moore, J.D., Brennan, J., Powers, M.A., and Kornbluth, S. (1998). Control of cyclin B1 localization through regulated binding of the nuclear export factor CRM1. Genes Dev 12, 2131-2143.

Yang, S.Z., Lin, F.T., and Lin, W.C. (2008). MCPH1/BRIT1 cooperates with E2F1 in the activation of checkpoint, DNA repair and apoptosis. EMBO Rep 9, 907-915. 
Yingling, J., Youn, Y.H., Darling, D., Toyo-Oka, K., Pramparo, T., Hirotsune, S., and Wynshaw-Boris, A. (2008). Neuroepithelial stem cell proliferation requires LIS1 for precise spindle orientation and symmetric division. Cell 132, 474-486.

Yoon, K., Nery, S., Rutlin, M.L., Radtke, F., Fishell, G., and Gaiano, N. (2004). Fibroblast growth factor receptor signaling promotes radial glial identity and interacts with Notch1 signaling in telencephalic progenitors. J Neurosci 24, 9497-9506.

Yu, T.W., Mochida, G.H., Tischfield, D.J., Sgaier, S.K., Flores-Sarnat, L., Sergi, C.M., Topcu, M., McDonald, M.T., Barry, B.J., Felie, J.M., et al. (2010). Mutations in WDR62, encoding a centrosome-associated protein, cause microcephaly with simplified gyri and abnormal cortical architecture. Nat Genet 42, 1015-1020.

Yu, X., Chini, C.C., He, M., Mer, G., and Chen, J. (2003). The BRCT domain is a phosphoprotein binding domain. Science 302, 639-642.

Zhang, J., Cicero, S.A., Wang, L., Romito-Digiacomo, R.R., Yang, Y., and Herrup, K. (2008). Nuclear localization of Cdk5 is a key determinant in the postmitotic state of neurons. Proc Natl Acad Sci U S A 105, 8772-8777.

Zhong, W., and Chia, W. (2008). Neurogenesis and asymmetric cell division. Curr Opin Neurobiol 18, 4-11.

Zhong, X., Liu, L., Zhao, A., Pfeifer, G.P., and Xu, X. (2005). The abnormal spindle-like, microcephaly-associated (ASPM) gene encodes a centrosomal protein. Cell Cycle 4, 12271229.

Zhong, X., Pfeifer, G.P., and Xu, X. (2006). Microcephalin encodes a centrosomal protein. Cell Cycle 5, 457-458.

Zimmerman, L., Parr, B., Lendahl, U., Cunningham, M., McKay, R., Gavin, B., Mann, J., Vassileva, G., and McMahon, A. (1994). Independent regulatory elements in the nestin gene direct transgene expression to neural stem cells or muscle precursors. Neuron 12, 11-24.

Zimmerman, W.C., Sillibourne, J., Rosa, J., and Doxsey, S.J. (2004). Mitosis-specific anchoring of gamma tubulin complexes by pericentrin controls spindle organization and mitotic entry. Mol Biol Cell 15, 3642-3657. 


\section{Appendix}

\subsection{Abbreviation list}

\begin{tabular}{|c|c|}
\hline BrdU & Bromodeoxyuridine \\
\hline BRCT & C-terminal domain of breast cancer gene 1 \\
\hline BSA & Bovine serum albumin \\
\hline CCD & Charge-coupled device \\
\hline CNS & Central nervous system \\
\hline $\mathrm{CP}$ & Cortical plate \\
\hline DAPI & 4',6-Diamidino-2-phenylindole \\
\hline DIVn & Day in vitro $n$ \\
\hline EDTA & Ethylene-diamine-tetra-acetic acid \\
\hline EGFP & Enhanced green fluorescent protein \\
\hline En & Embryonic day $n$ \\
\hline ES cell & Embryonic stem cell \\
\hline FACS & Fluorescence activated cell sorting \\
\hline FCS & Fetal calf serum \\
\hline GABA & $\gamma$-aminobutyric-acid \\
\hline$\gamma$-TuRC & $\gamma$-tubulin ring complex \\
\hline $\mathrm{H} \& \mathrm{E}$ & Hematoxylin and eosin \\
\hline $\lg$ & Immunglobulin \\
\hline IPC & Intermediate progenitor cell \\
\hline IZ & Intermediate zone \\
\hline IQ & Intelligence quotient \\
\hline $\mathrm{MCPH}$ & Primary microcephaly \\
\hline MEF & Mouse embryonic fibroblasts \\
\hline $\mathrm{MZ}$ & Marginal zone \\
\hline NE & Neuroepithelial cell \\
\hline NMRI & Nuclear magnetic resonance imaging \\
\hline
\end{tabular}


OFC Occipito-frontal head circumference

PBS Phosphate buffered saline

PCR Polymerase chain reaction

PFA Paraformaldehyde

Pn Postnatal day $n$

PP Preplate

PVDF Polyvinylidene fluoride

RGC Radial glial cell

rpm Rounds per minute

RT Room temperature

SD Standard deviation

SDS Sodium dodecyl sulfate

S.e.m Standard error of mean

SNP Short neural precursor

SP Subplate

SVZ Subventricular zone

TEMED Tetra-methyl-ethylene-diamine

TUNEL Terminal deoxynucleotidyl transferase dUTP nick end labeling

VZ Ventricular zone 


\subsection{Acknowledgements}

First of all, I want to thank my supervisor Prof. Dr. Zhao-Qi Wang for giving me the opportunity to work in his laboratory and to work on this exciting project. I am deeply grateful to him for his constant help and scientific advice. I really appreciate that he showed always interest in my project. His interest and help in every experimental result finally made it possible to bring this study to its current end.

I would also like to thank PD Dr. Wilfried Kramer for being a reviewer of my Thesis. I am very grateful that he agreed to this, because without him I could not even start my Ph.D. I am also very grateful that he always found the time to travel from Göttingen to Jena to participate in my Ph.D. committee meetings and to give advice on my project.

I would like to thank Dr. Eberhard Fritz who supervised me during my Diploma Thesis and continued to help me as a member of my Ph.D. committee. I am grateful that he found the time to participate in my regular Ph.D. committee meetings and for his advice.

I am extremely thankful to Dominique Galendo, Christof Birch-Hirschfeld and Cathrin Müller who took care of my mouse colonies. All of you did an excellent work.

I would like to thank Maik Baldauf and Kathrin Buder for the histological service also I want to thank Anne Gompf for her professional work on the FACsorting.

I want to thank Mikhail Sukchev who did the gene targeting in ES cells to generate the Mcph1knockout mice and I would like to thank Zhongwei Zhou for his work on the MCPH1-project. Also I want to thank both of you for the many helpful discussions.

I would like to thank Tjard Jörß, Anja Krüger and Mareen Welzel for their helpful technical assistance and also for creating a very nice atmosphere in the lab. I also would like to thank Tjard for the Kuli once in a while, because it is impossible to finish a Ph.D. without this. 
I want to thank Alicia Tapias Soler for her reading and helpful corrections of my Thesis and I also would like to thank her for not complaining too much. I am also very grateful for the many and very helpful brain-discussions.

I am very thankful to all members of the Wang's lab for their help and discussions and for creating such a great atmosphere in the lab: Christopher Bruhn, Kathrin Eberhard, Fu Li, Tangliang Li, Yu-Chieh Lin, Haizhen Liu, Yu-Feng Liu, Wookee Min, Mara Sannai, Dana Schoder, Amal Saidi, Benazir Siddek, Laura Perucho Aznar and Sandra Orthaus.

My most special thanks are for my girlfriend Valentina. It would have been impossible to finish my Thesis, if she had not forgotten me in the library. Un caro ringraziamento a Valentina.

Finally, I would like to express my greatest gratitude to my parents and my two brothers. Ich möchte Euch danken, dass Ihr mich immer unterstützt habt und stets für mich da wart. Danke! 


\subsection{Curriculum Vitae}

\section{Ralph Gruber}

Leibniz-Institute for Age Research - Fritz-Lipmann-Institute e.V.

Beutenbergstr. 11

D-07745 Jena, Germany

Phone: +49-3641-656419

E-mail: rgruber@fli-leibniz.de

Date of birth: June $27^{\text {th }}, 1979$

Place of birth: Illertissen

Citizenship: German

\section{Education}

Since 2007 Leibniz-Institute for Age Research - Friz-Lipmann-Insitute e.V., Jena, Germany. Ph.D. Thesis in the laboratory of Prof. Dr. Zhao-Qi Wang.

2006-2007 Leibniz-Institute for Age Research - Friz-Lipmann-Insitute e.V., Jena, Germany. Scientific Assistant in the laboratory of Prof. Dr. Peter Herrlich.

2005-2006 Leibniz-Institute for Age Research - Friz-Lipmann-Insitute e.V., Jena, Germany. Diploma Thesis in the laboratory of Prof. Dr. Peter Herrlich under supersivion of Dr. Eberhard Fritz and Dr. Anke Schuerer.

2001-2006 University of Applied Sciences Weihenstephan, Freising, Germany. Course of studies: Biotechnology. 
$\underline{\text { Internship }}$

2004-2005 Shandong Agricultural University, Taian, P.R. China.

Internship in the Dept. Molecular Biology - Laboratory of Prof. Zheng.

Vocational education and working experience

1996-1999 Wasserwirtschaftsamt Donauwoerth, Germany.

Vocational training as Chemical Laboratory Assistant.

1999-2000 Wasserwirtschaftsamt Donauwoerth, Germany.

Employment as Chemical Laboratory Assistant.

$\underline{\text { School education }}$

2000-2001 Highschool (Berufsoberschule), Donauwoerth, Germany.

1994-1996 Primary school (Private Wirtschaftsschule), Donauwoerth, Germany 


\section{List of publications}

Ronit Galron, Ralph Gruber, Veronica Lifshitz, Haizhen Lu, Michal Kirshner, Natali Ziv, ZhaoQi Wang, Yosef Shiloh, Ari Barzilai and Dan Frenkel. Glial cell dysfunction enhanced cerebellar attrition in a Nijmegen Breakage Syndrome animal model (2011). J Mol Neurosci.

Susanne A. Gatz, Cong Liu, Limei Ju, Ralph Gruber, Eva Hoffmann, Antony Carr, Zhao-Qi Wang and Penny A. Jeggo. Requirement for DNA ligase IV during embryonic development. (2011). J Neurosci.

Manuscript accepted.

Ralph Gruber, Mikhail Sukchev, Zhongwei Zhou, Tjard Joerss, Pierre-Olivier Frappart and Zhao-Qi Wang. MCPH1 regulates the neuroprogenitor division mode by coupling the centrosome cycle with mitotic entry via the Chk1-Cdc25 pathway (2011).

Manuscript in revision.

Ralph Gruber, Haizhen Lu, Tjard Joerss and Zhao-Qi Wang. Deletion of Nbs1 in Purkinje cells revealed a dispensable function of DNA damage in response in postmitotic cells (2011). Manuscript in preparation.

\section{Presentations}

Multidisciplinary Conference: Lifestyle and Ageing. October 4.-5. 2010. Pisa, Italy.

\section{Oral presentation.}

11th Biennual DGDR Meeting: DNA repair. September 7.-10. 2010. Jena, Germany. Oral and poster presentation. 
International Summer School: From Pluripotency to Senescence. Molecular Mechansims of Development, Disease and Ageing. August 21.-30. 2010. Island of Spetses, Greece.

Oral and poster presentation (poster prize).

Maintenance of Genome Stability. March 8.-11. 2010. Antigua.

Poster presentation.

2nd German-French DNA Repair Meeting. September 20.-23. 2009. Konstanz, Germany. Oral presentation.

Genome Stability in Health and Disease. September 21.-25. 2008. Jerusalem, Israel. Poster presentation.

Recombination and Repair Conference. September 16.-17. 2005. Hamburg, Germany. Poster presentation. 